Portland State University

PDXScholar

1990

\title{
The Effect of Two Viologens on the Solution Speciation of Tetrakis([rho]-carboxyphenyl)porphine
}

Suzanne Elizabeth Clarke

Portland State University

Follow this and additional works at: https://pdxscholar.library.pdx.edu/open_access_etds

Let us know how access to this document benefits you.

\section{Recommended Citation}

Clarke, Suzanne Elizabeth, "The Effect of Two Viologens on the Solution Speciation of Tetrakis([rho]carboxyphenyl)porphine" (1990). Dissertations and Theses. Paper 1372.

https://doi.org/10.15760/etd.1371

This Dissertation is brought to you for free and open access. It has been accepted for inclusion in Dissertations and Theses by an authorized administrator of PDXScholar. Please contact us if we can make this document more accessible: pdxscholar@pdx.edu. 
THE EFFECT OF TWO VIOLOGENS ON THE SOLUTION SPECIATION OF TETRARIS ( $p$-CARBOXYPHENYL) PORPEINE

by

SUZANNE ELIZABETH CLARKE

A dissertation submitted in partial fulfillment of the requirements for the degree of

\author{
DOCTOR OF PHILOSOPHY \\ in \\ ENVIRONMENTAL SCIENCES AND RESOURCES: \\ CHEMISTRY
}

Portland State University

(C) 1990 
TO THE OFFICE OF GRADUATE STUDIES:

The members of the Committee approve the dissertation of suzanne Elizabeth Clarke presented May 8, 1990.

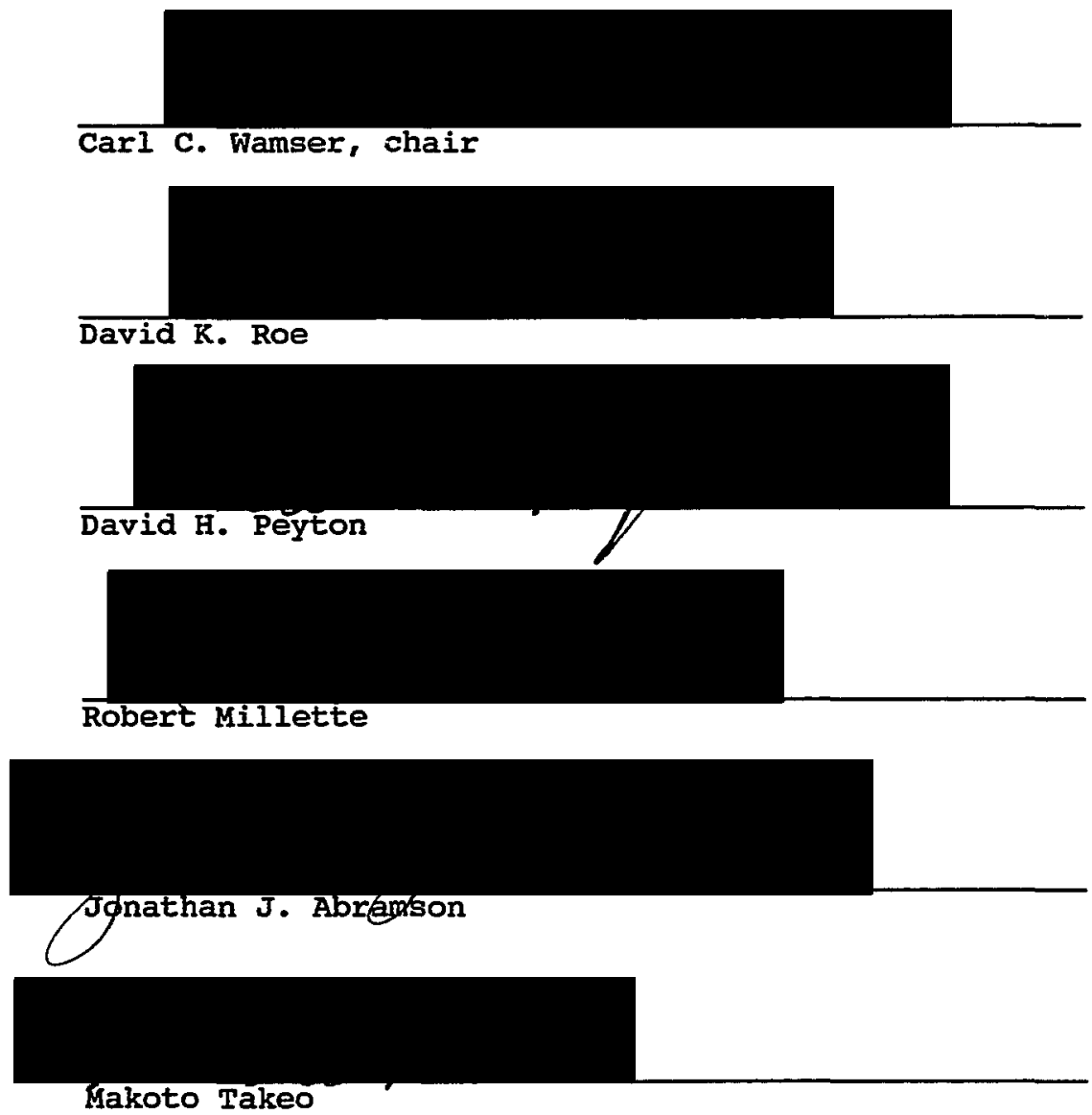

\section{APPROVED :}

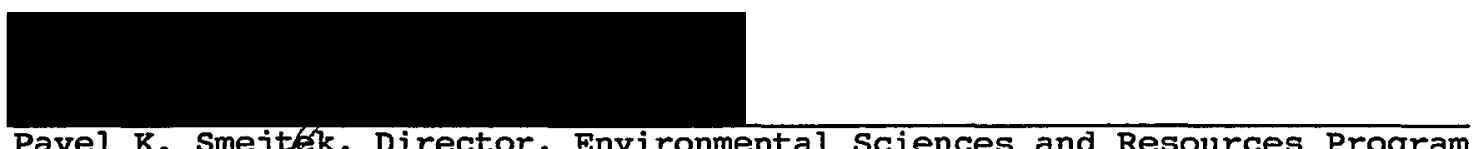

Pavel K. Smejtek, Director, Environmental Sciences and Resources Program

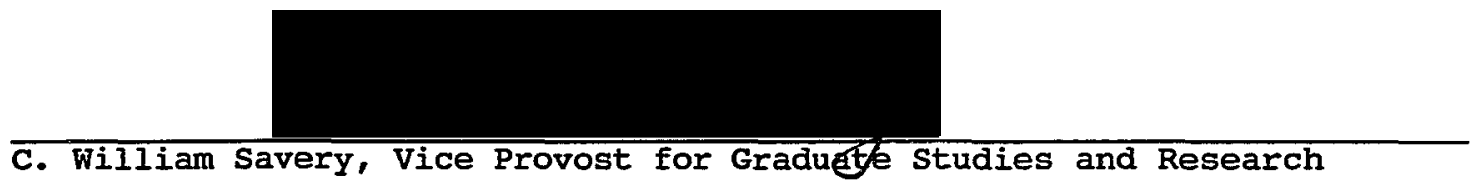


AN ABSTRACT OF THE DISSERTATION OF Suzanne Elizabeth clarke for the Doctor of Philosophy in Environmental sciences and Resources/Chemistry presented May 8, 1990.

Title: The Effect of Two Viologens on the solution speciation of Tetrakis ( $p$-carboxyphenyl) porphine.

APPROVED BY THE MEMBERS OF THEE DISSERTATION COMMITTEE:

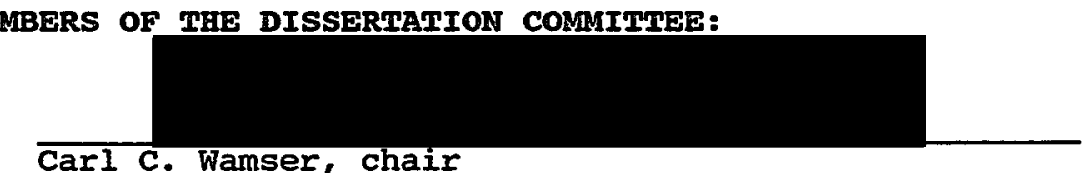

Carl c. Wamser, chair
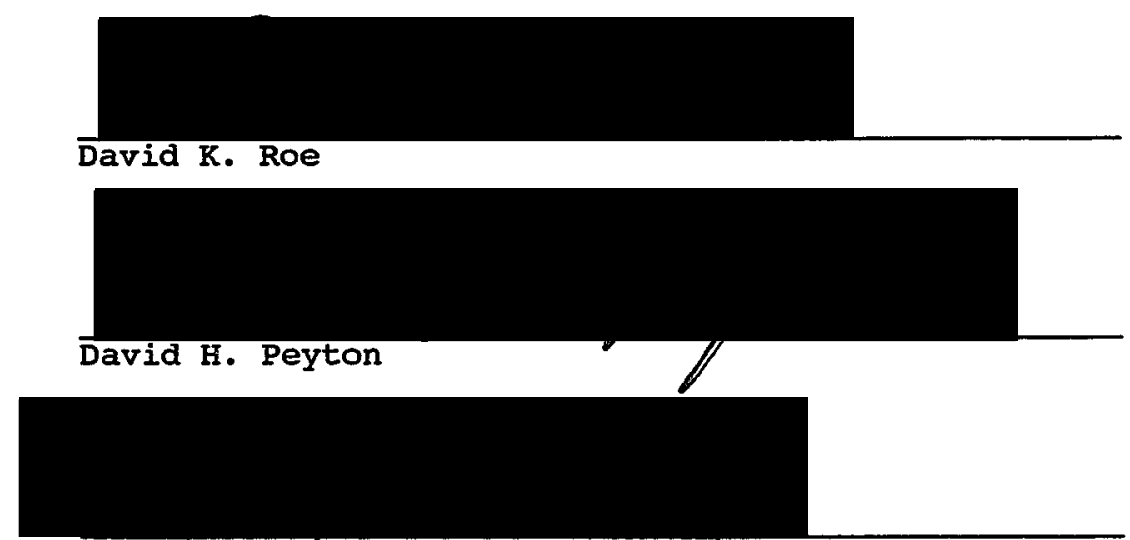

Robert I. Millette

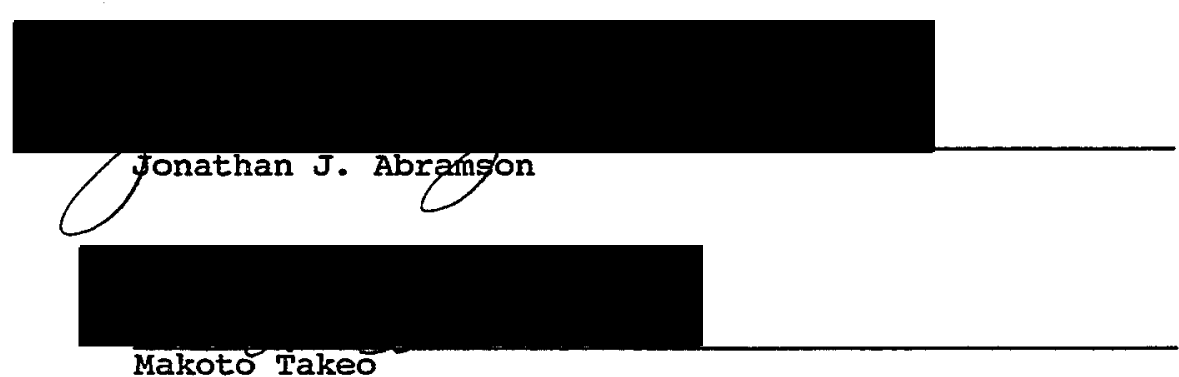

Porphyrins and viologens are often utilized in photocatalyzed electron transfer chemistry. Some of the problems faced by researchers in these applied studies include the lack of information correlating the porphyrin 
absorbance spectral changes with porphyrin dimerization, complexation, and the effect of noncomplexing cations. In addition, the efficiency of the system is greatly reduced by the formation of porphyrin:viologen association complexes. Knowledge of the values of these constants may allow selection of appropriate porphyrin:viologen concentration ratios which would enable optimization of these systems when used in such applications as artificial photosynthesis. This study reports the effect of selected salts and solvents on the porphyrin spectrum and the spectrum of the $\mathrm{H}_{2} \mathrm{TCPP}^{4-}$ dimer. Moreover, we define experimental conditions appropriate to the study of porphyrin:viologen association and report association constants calculated at both constant ionic strength and constant buffer concentration.

The spectrophotometric titration of tetrakis(pcarboxyphenyl)porphine $\left(\mathrm{H}_{2} \mathrm{TCPP}^{4-}\right)$ with either methyl viologen $\left(\mathrm{MV}^{2+}\right)$ or propylviologen sulfonate $\left(\mathrm{PVS}^{0}\right)$ results in the formation of $\mathrm{H}_{2} \mathrm{TCPP}^{4-}$ : viologen complexes with stoichiometries of both $1: 1$ and $1: 2$. In addition, at high viologen concentration, $\mathrm{PVS}^{\mathrm{O}}$ induces $\mathrm{H}_{2} \mathrm{TCPP}^{4-}$ dimerization. Association constants for the titration of $\mathrm{H}_{2} \mathrm{TCPP}^{4-}$ with $\mathrm{MV}^{2+}$ were calculated using data obtained at constant ionic strength (I = 0.15 , maintained with $\mathrm{KH}_{2} \mathrm{PO}_{4} / \mathrm{NaKHPO}_{4}$ ) and at constant buffer concentration $\left(\left[\mathrm{KH}_{2} \mathrm{PO}_{4} / \mathrm{NaKHPO}_{4}\right]=5 \mathrm{mM}\right)$ using both nonlinear leastsquares (NLLS) and principal component analysis (PCA). At constant buffer concentration the association constants calculated via NLIS analysis and PCA were found to be, respectively, 3,170 and 3,350 for the 1:1 complex and 100 and 68 for the 2:1 complex. At constant ionic strength the association constants were calculated by NLLS to be 594 for the 1:1 complex and 38 for the 2:1 complex.

PCA was used to confizm our model of the solution equilibrium equations used to calculate the association constants by NLLS analysis. In addition, this method yields the spectral line shape and absorptivity of the spectroscopically unresolved $1: 1 \mathrm{H}_{2} \mathrm{TCPP}^{4-}: \mathrm{MV}^{2+}$ complex. From this 
information it was learned that both the absorptivity and the wavelength dependence of $\mathrm{H}_{2} \mathrm{TCPP}^{4-}$ and the viologen complexes of $\mathrm{H}_{2} \mathrm{TCPP}^{4-}$ were a function of the alkali metal cation concentration (higher concentrations of cations result in progressively weakened and blue-shifted spectra). Finally, we will present data which support the conclusion of Firman et al. that the spectrum of the $\mathrm{H}_{2} \mathrm{TCPP}^{4-}$ dimer is weakened and blueshifted by approximately $10 \mathrm{~nm}$ relative to the porphyrin monomer in aqueous solution. We will further show that some organic ions induce $\mathrm{H}_{2} \mathrm{TCPP}^{4-}$ dimerization and some do not; moreover, we will present experimental evidence demonstrating that the data cannot be explained as a simple effect of the ionic strength. 
TABLE OF CONTENTS

PAGE

IIST OF TABLES . . . . . . . . . . . . . . . . . . . . . . . vi

IIST OF FIGURES . . . . . . . . . . . . . . . . . . . . . . . . . vii

\section{CHAPTER}

I INTRODUCTION . . . . . . . . . . . . . . . . . . . 1

Overview of the Literature . . . . . . . . . . . . 1

Environmental Significance . . . . . . . . . . . 9

Contributions of This Study . . . . . . . . . . . 13

II PORPHYRIN SPECTROSCOPY: VISIBLE AND NMR . . . . . . . . . . 15

Introduction . . . . . . . . . . . . . . . . 15

Visible Absorbance Spectroscopy . . . . . . . . . 17

$\mathrm{D}_{\text {4h }}$ Symmetry . . . . . . . . . . . . . . . . 17

$\mathrm{D}_{\mathrm{h}}$ Symmetry ...................... . 21

Nuclear Magnetic Resonance Spectroscopy . . . . . . 35

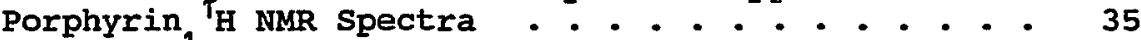

Viologen ${ }^{1}$ H NMR Spectra .............. . 39

III MATERIALS AND METHODS . . . . . . . . . . . . . . . . . 42

Introduction . . . . . . . . . . . . . . . . . . . 42

Synthesis and/or Purification of Materials . . . . . . . . 42

Purification of Commercial Porphyrins . . . . . . . . . 42

Synthesis of $\mathrm{H}_{2} \mathrm{TAPP}^{4+}$. . . . . . . . . . . . . . . . . 43

Purification of Commercial Viologens . . . . . . . . . 49

Synthesis and Purification of PVS $^{0}$. . . . . . . . . . 51

Salts, Buffers, and Detergents . . . . . . . . . . 53

Instrumentation . . . . . . . . . . . . . . . . . . . 53

UV/VIS Absorbance Spectrophotometer . . . . . . . . 53

Nuclear Magnetic Resonance Spectrometers . . . . . . . 54

Temperature Regulation . . . . . . . . . . . . . . . 54

Temperature Measurement . . . . . . . . . . . . 55

Absorbance Measurements: Methodology . . . . . . . . . . 56

Introduction . . . . . . . . . . . . . . . . . . 56

Stock Solutions . . . . . . . . . . . . . . . . . 56

Method 1. Individually Prepared Solutions . . . . . . 59

Method 2. Spectrophotometric Titrations . . . . . . . . 61 
Nuclear Magnetic Rescnance Measurements: Methodology . . . 67

Preparation of Solutions . . . . . . . . . . . . 67

Data Analysis . . . . . . . . . . . . . . . . 68

Cubic spline Calibration Curve for Thermistor Temperature Measurements . . . . . . . . . . 68

Determination of Solution Parameters by Least-squares Methods ...................... 68

Speciation Determination Technique Using Principle Component Analysis . . . . . . . . . . . 70

IV THE EFFECT OF SALTS AND SOLVENTS ON THE SPECTRUM AND

SPECIATION OF $\mathrm{H}_{2} \mathrm{TCPP}^{0}\left(\mathrm{H}_{2} \mathrm{TCPP}^{4-}\right) \cdot . \cdot . \cdot . \cdot . \cdot . \cdot . \cdot-75$

Introduction . . . . . . . . . . . . . . . . 75

Summary of the Data Presented in this Chapter . . . . . 78

The Effect of Organic Salts on the Induced Dimerization and Dimer Dissociation of the Anionic Tetraphenylporphyrins .. . . . . . . . . . . 83

The Effect of CTAB on the spectra and speciation of $\mathrm{H}_{2} \mathrm{TSPP}^{4-}$. . . . . . . . . . . . . . . . . 84

MMR Evidence for Association of $\mathrm{H}_{2} \mathrm{TCPP}^{4-}$ with $\mathrm{MV}^{2+}$ : Dissociation of $\mathrm{H}_{2} \mathrm{TCPP}^{4-}$ Dimers with $\mathrm{MV}^{2+} \cdot$. . . . $90^{-} 9$

Summary . . . . . . . . . . . . . . . . 93

The Effect of Inorganic Cation Concentration on the Beer's Law Plots . . . . . . . . . . . . 97

Spectral Iine Shape Changes Induced by Low Buffer Concentration ...... . . . . . . . . . . 104

V DETERMINATION OF THE STOICHIOMETRY AND ASSOCIATION CONSTANTS

FOR THE COMPLEXATION OF H2TCPP ${ }^{4-}$ WITH $\mathrm{MV}^{2+}$. . . . . . 116

Review of the Literature . . . . . . . . . . . 119

Determination of Association Constants . . . . . . 123

Strategy for calculating equilibrium constants . . . 125

Methods and Results of NLIs Analyses, Constant Buffer Concentration . . . . . . . . . . . . . 126

Methods and Results of NLLS Analysis, Constant Ionic Strength .................... 130

Evidence for $\mathrm{H}_{2} \mathrm{TCPP}^{4-}: \mathrm{MV}^{2+}$ Complexes With stoichiometries of $1: 1$ and $1: 2$..................

Methods and Analysis by PCA, Constant Buffer Concentration . . . . . . . . . . . . . 140

Analysis by PCA, Constant Ionic strength . . . . . . 148 
SELECTED REEERENCES . . . . . . . . . . . . . . . . . . . . 


\section{IIST OF TABLES}

TABLE

PAGE

I Porphyrins Used in This Study . . . . . . . . . . . . . 5

II Viologens Used In This Study . . . . . . . . . . . . . 11

III Comparison of Three Instrumental Methods . . . . . . . 16

IV Comparison of Two Methods Used in the Absorbance Studies 56

V Spectrophotometric Titrations: [Buret] versus [Cuvette] 62

VI The Effect of Ethylene Glycol:water solutions on the Porphyrin Spectrum . . . . . . . . . . . . 81

VII Solutions Used in the $300 \mathrm{MHz}^{1} \mathrm{H}$ NMR study of $\mathrm{H}_{2} \mathrm{TCPP}^{4-}$

$/ \mathrm{MV}^{2+}$ Complexation . . . . . . . . . . . . . 91

VIII Beer's Law Experiments: Summary of Experimental Conditions . . . . . . . . . . . . . 99

IX Beer's Law Experimental Results . . . . . . . . . . 100

X Summary of Reported Equilibrium Constants . . . . . . 118

XI Model Used in The NLLS Analysis . . . . . . . . . . . 134

XII Data Summary: Titration of $\mathrm{H}_{2} \mathrm{TCPP}^{4-}$ With $\mathrm{MV}^{2+}$. . . . . 134

XIII Equilibrium Constants Calculated by NLLS and PCA • . 155 


\section{LIST OF FIGURES}

$\begin{array}{ll}\text { FIGURE } & \text { PAGE }\end{array}$

1. Resonance of the porphine macrocycle in $D_{4 h}$ symmetry. . . . 18

2. Spectrum of $\mathrm{H}_{4} \mathrm{TAPP}^{6+}$ in acidic aqueous solution . . . . . . . 20

3. Resonance of the porphine macrocycle in $\mathrm{D}_{2 \mathrm{~h}}$ symmetry. . . . 21

4. Spectrum of $\mathrm{H}_{2} \mathrm{TCPP}^{0}$ in tetrahydrofuran . . . . . . . . . . . . 22

5. Spectrum of $1 \mu \mathrm{M} \mathrm{H} \mathrm{H}_{2} \mathrm{TCPP}^{4-}$ in aqueous solution buffered to $\mathrm{pH}=7$ with $5 \mathrm{mM}$ phosphate. . . . . . . . . . . . . . . . 23

6. Spectra of $\mathrm{H}_{2} \mathrm{TCPP}^{0}$ in dimethylsulfoxide (DMSO) and chloroform $\left(\mathrm{CHCl}_{3}\right)$. . . . . . . . . . . . . . . . . . . 27

7. Spectrum of $\mathrm{H}_{2} \mathrm{TAPP}^{0}$ in tetrahydrofuran. . . . . . . . . . . . 28

8. Spectra of $\mathrm{H}_{2} \mathrm{TAPP}^{0}$ in dimethylsulfoxide and chloroform. . . $\quad 29$

9. Possible rationalization for the solvent effects observed in $\mathrm{H}_{2} \mathrm{TAPP}^{0}$ but not in $\mathrm{H}_{2} \mathrm{TCPP}^{4-}$. . . . . . . . . . . . . . . 30

10. Spectra of $\mathrm{H}_{4} \mathrm{TAPP}^{6+}$ and $\mathrm{H}_{2} \mathrm{TAPP}^{\text {?+ }}$ in acidic aqueous solution. $\quad 31$

11. Spectra of $\mathrm{H}_{2} \mathrm{TAPP}^{0}$ in chloroform and $\mathrm{H}_{\text {? }} \mathrm{TAPP}^{\text {?+ }}$ in acidic chloroform. . . . . . . . . . . . . . . . . . .

12. Spectra of $\mathrm{H}_{2} \mathrm{TSPP}^{4-}$ in weakly acidic and weakly basic aqueous solutions.

13. $300 \mathrm{MHz}{ }^{1} \mathrm{H}$ NMR spectrum of $\mathrm{H}_{2} \mathrm{TCPP}^{4-}$ in $\mathrm{D}_{2} \mathrm{O}$ at $\mathrm{pD}=7$, buffered with $5 \mathrm{mM} \mathrm{KD} \mathrm{PO}_{4} / \mathrm{NaKDPO}_{4} \cdot$. . . . . . . . . . . . . . 38

14. $90 \mathrm{MHz}{ }^{1} \mathrm{H}$ NMR spectra of $\mathrm{MV}^{2+}$ and $\mathrm{mMV}^{1+}$ in $\mathrm{D}_{2} \mathrm{O}$. . . . . . . . . 40

15. $90 \mathrm{MHz}^{1} \mathrm{H} \mathrm{NMR}$ spectra of $\mathrm{PVS}^{0}$ and $\mathrm{MPVS}^{0}$ in $\mathrm{D}_{2} \mathrm{O}$. . . . . . . . 41

16. Overall reaction in the synthesis of a substituted tetraphenylporphyrin and two of the possible side-reactions. . 45

17. A plausible mechanism for the synthesis of $\mathrm{H}_{2} \mathrm{TAPP}^{4+}$. . . .46

18. Plausible mechanism for the synthesis of PVs $^{0}$. . . . . . . . 51

19. Thermistor Calibration curve. . . . . . . . . . . . . . 55

20. Diagram of Heat Exchanger for Variable Pathlength Cells. . . 60 
21. Diagram of the titration apparatus which inserts into the spectrophotometer. . . . . . . . . . . . . . . . 66

22. Flow diagram illustrating the steps involved in principal component analysis. . . . . . . . . . . . . . . . . . 74

23. Titration of $\mathrm{H}_{2} \mathrm{TCPP}^{4-}$ with A) $\mathrm{MV}^{2+}$ and B) $\mathrm{PVS}^{0}$. . . . . . . 76

24. The spectrum of $\mathrm{H}_{2} \mathrm{TCPP}^{4-}$ in different water:ethylene glycol mixtures. . . . . . . . . . . . . . . . . . 80

27. Spectrum of $\mathrm{H}_{2} \mathrm{TCPP}^{4-} /$ heterodimers. . . . . . . . . . . . . . 87

28. Dissociation of dimerized $\mathrm{H}_{2} \mathrm{TSPP}^{4-}$ by the addition of 15-crown5 ether. . . . . . . . . . . . . . . . . . 89

29. $300 \mathrm{MHz}{ }^{1} \mathrm{H}$ NMR study of $\mathrm{H}_{2} \mathrm{TCPP}^{4-} / \mathrm{MV}^{2+}$ association. . . . . . 92

31. Illustration of two possible orientations for the propylsulfonate group in the $\mathrm{H}_{2} \mathrm{TCPP}^{4-} / \mathrm{PVS}^{\mathrm{O}}$ association complex. . . . . . . . . . . . . . . . . . . .

32. The effect of minimal ionic strength and low pH on the Beer's law spectra of $\mathrm{H}_{2} \mathrm{TSPP}^{4-}$. . . . . . . . . . . . . . 102

33. Beer's law plots of increasing concentration of $\mathrm{H}_{2} \mathrm{TCPP}^{4-}$ at $\mathrm{pH}$ $=10$ and minimal ionic strength. . . . . . . . . . . 105

36. Comparison of the spectra of $1 \mu \mathrm{M} \mathrm{H} \mathrm{HCPP}^{4-} 0.5 \mathrm{mM}$ salt (pH $\approx$ 9.5), 5 and $62 \mathrm{mM}$ phosphate $(\mathrm{pH}=7.0) . . . . . . .$.

37. The effect of increasing phosphate concentration on the absorbance of $1.00 \mu \mathrm{M} \mathrm{H}_{2} \mathrm{TCPP}^{4-}$ at $414.2 \mathrm{~nm}$. . . . . . . 110

38. The effect of low phosphate concentration on the $\mathrm{H}_{2} \mathrm{TCPP}^{4-}$ absorbance at $414.2 \mathrm{~nm}$. . . . . . . . . . . .

39. Gaussian decomposition of the phosphate dependence spectral

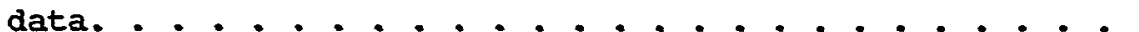

41. Spectrophotometric titration of $\mathrm{H}_{2} \mathrm{TCPP}^{4-}$ with $\mathrm{MV}^{2+}$, experiment 1. . . . . . . . . . . . . . . . . . . .

42. Spectrophotometric titration of $\mathrm{H}_{2} \mathrm{TCPP}^{4-}$ with $\mathrm{MV}^{2+}$, experiment 2........................ 
43. Spectrophotometric titration of $\mathrm{H}_{2} \mathrm{TCPP}^{4-}$ with $\mathrm{MV}^{2+}$, experiment 3. . . . . . . . . . . . . . . . . . . . 131

44. Titration of $\mathrm{H}_{2} \mathrm{TCPP}^{4-}$ with $\mathrm{MV}^{2+}$, experiment $1 . \quad$. . . . . $\quad$. 135

45. Titration of $\mathrm{H}_{2} \mathrm{TCPP}^{4-}$ with $\mathrm{MV}^{2+}$ experiment 3 ; spectra and difference spectra. . . . . . . . . . . . . . . 138

46. Titration of $\mathrm{H}_{2} \mathrm{TCPP}^{4-}$ with $\mathrm{MV}^{2+}$; experiment 4 . . . . . . . .139

47. Three principal components in the spectral data from experiment 2. • . . . . . . . . . . . . . . . . . 141

48. Spectra which pass through the first and second isosbestic points with their corresponding eigenvalue plots. . . .

49. Eigenvector coefficient plot used to derive the line shapes and absorptivities for $\mathrm{H}_{2} \mathrm{TCPP}^{4-}$ and the $\mathrm{H}_{2} \mathrm{TCPP}^{4-}: \mathrm{MV}^{2+}$ complexes. . . . . . . . . . . . . . . . . .

50. Spectral line shapes of the three absorbing components in experiment 2 from PCA analysis. . . . . . . . . . .

51. Speciation plot comparing the PCA with the NLLs analysis for experiment 2. . . . . . . . . . . . . . . . . .

52. Three principal components in the spectral data from experiment 3. . . . . . . . . . . . . . . . . .

53. Spectra which pass through the first and second isosbestic points with their corresponding eigenvalue plots. . . .

54. Eigenvector coefficient plot used to derive the line shapes and absorptivities for $\mathrm{H}_{2} \mathrm{TCPP}^{4-}$ and the $\mathrm{H}_{2} \mathrm{TCPP}^{4-}: \mathrm{MV}^{2+}$ complexes. . . . . . . . . . . . . . . . . .

55. Spectral line shapes of the three absorbing components in experiment 3 from PCA analysis. . . . . . . . . . .

56. Speciation plot comparing the PCA witn the NLLS analysis for experiment 3. . . . . . . . . . . . . . . . . . 


\section{CHAPTER I}

\section{INTRODUCTION}

\section{OVERVIEW OF THE IITERATURE}

Porphyrin derivatives are ubiquitous in biochemical systems and are arguably the most significant organic moiety used as a cofactor. They are found in such critical biological molecules as cytochrome $c$, hemoglobin, cytochrome P450, chlorophyll and cobalamin (vitamin $\mathrm{B}_{12}$ ). In each case, the active site is the metal-chelated porphyrin, catalyzing: 1) electron-transport, 2) reversible oxygen binding, 3) chemical syntheses, or 4) photo-catalyzed redox processes. (1-16) The chronology of porphyrin research might be loosely viewed as containing two eras. In an early "theoretical" era synthetic chemists (17-26) worked in conjunction with molecular spectroscopists, physical and bioinorganic chemists $(1-16,27-32)$ to develop a physical understanding of porphyrin-mediated processes. In the present "applied" era porphyrins are studied for their ability to function in some practical application such as solar energy conversion (33-71), trace metal analysis $(72,73)$, or tumor therapy $(74-82)$. The intense interest in porphyrins over the last thirty years has resulted in a huge and divergent body of literature. Those aspects of the literature most pertinent to this work are briefly discussed below and will be viewed in greater detail in later chapters.

Much early work focused on building model compounds in an attempt to understand the mechanisms of various porphyrin-catalyzed reactions in systems less complex than "in vivo" (17-26). Porphyrins are used in four types of biological systems. The first is oxygen transport. Collman $(21,22)$ and coworkers contributed much to the understanding of 
oxygen binding in hemoglobin and myoglobin with the elegant synthesis and studies of the "picket fence" porphyrins. The second is electron transfer. In biological systems, energy released from electron transfer down an electrochemical gradient is stored as chemical energy in such molecules as ATP. The electrochemical gradient is formed by an assembly of redox centers; many of the components are protein-bound metalloporphyrins. In this field Harry Gray $(4,9,16)$ and R.J.P. Williams $(2,5)$ have addressed such fascinating questions as how electrons jump from metal center to metal center. The third is the enormous field of porphyrin-catalyzed chemical reactions, a specific example being the liver enzymes which help to solubilize organic toxins via the addition of hydroxyl groups $(1-3,7)$. And the fourth, most closely related to this work, is the field of conversion of light energy to energy stored in chemical bonds, a process called photosynthesis $(6,10,15,33)$. The biological molecules involved in this process are the chlorophylls, which in nature occur as arrays in thylakoid membranes or bound into proteins. The relative spacial orientation of protein-bound chlorophylls can be precisely controlled by the protein ( 6 ).

The availability of model compounds is often critical to the understanding of complex biological processes. When good model compounds are obtainable, scientists can often correlate an isolated chemical structure to a specific biological function. Once this has been accomplished, it is possible to determine how variations in structure or other parameters effect the function. Chang and coworkers (61) have contributed much to understanding how the relative spacial orientation of porphyrins modifies their physical properties by synthesizing porphyrins linked covalently into dimers. The length of the spacers can be varied to imitate the three dimensional orientation of naturally occurring chlorophylls. These model compounds are very useful as one can correlate a known spacial proximity of porphyrin monomer units with spectroscopic and electrochemical properties. 
Biological photosynthetic systems are optimized for low light conditions (33); during high photon flux these systems use as little as 128 of the incoming light energy. This is due to slow turnover rate of one of the reductive enzymes. Although much of the other $88 \%$ is absorbed by the light-harvesting pigments, it is lost through fluorescence and "non-radiative" decay. Natural systems thrive because the enzyme systems catalyzing the splitting of water and the reduction of $\mathrm{CO}_{2}$ are very specific and yield few side reactions.

In addition to the problems associated with optimizing the kinetics, artificial photosynthetic systems are hampered by the absence of catalysts which can separate the oxidative and reductive processes (34). This results in the production of mixed products $\left(\mathrm{O}_{2}\right.$ and $\left.\mathrm{H}_{2}\right)$ which must be separated by energy consuming techniques. Optimizing artificial systems utilizing porphyrins requires optimizing the light harvesting properties (maximizing absorbance) and minimizing energy loss (non-radiative decay and fluorescence). Dimerization in "in vitro" systems lowers light harvesting efficiency as the absorptivity of the dimer in solution is lower than that of the porphyrin monomer (60-69).

A major obstacle to "in vitro" studies of porphyrins is their poor aqueous solubility resulting in dimerization or the formation of higher aggregates which can lead to precipitation. The shape of porphyrin macrocycle is roughly discoid and results in two discrete structural possibilities for the porphyrin dimer, face-to-face and edge-to-edge. Many intermediate structures can be visualized for each by varying the angle between the two faces. In addition, face-to-face dimers could be offset. This can be visualized by fixing one porphyrin in space and allowing the second porphyrin to slide over face of the stationary molecule.

The tetraphenylporphyrins (TPP's) were designed (17-25) to reduce dimerization and to increase porphyrin aqueous solubility by attaching derivatized phenyl groups (charged) to the methine bridge (Table I). It 
was intended that the phenyl groups would reduce dimerization in two ways: 1) through steric effects, because the phenyl groups are twisted out of the porphyrin plane, which should reduce face-to-face associations of the planar porphyrin macrocycle; and 2) through the charged groups which should both limit both face-to-face and edge-toedge dimerization via coulombic repulsion and increase association of the porphyrin moiety with the polar solvent. However, studies by Pasternack and coworkers (83-85) showed that the aqueous solubility of the substituted tetraphenylporphyrins varied and depended upon the identity of the substitution and the presence and identity of a central metal ion.

The biologically relevant porphyrins are bound to metals. The presence of a metal influences the porphyrin's solution behavior via the charge on the metal ion and through axial ligation. In oxic solutions many metalloporphyrins form face-to-face dimers by axial ligation to a common oxygen atom in a structure called a $\mu$-oxo dimer. A "free base" porphyrin is one in which the metal binding site is in the "acid" form, i.e. " $\mathrm{H}_{2}$ " Studies of the free base porphyrins are much less prevalent yet important in separating the role of the metal ion from the role of the chromophore.

The free base tetraphenylporphyrin (TPP) unit can be substituted (at the phenyl group) to yield either anionic or cationic molecules whose water solubility (83-89) depends upon the polarity of the functional group and the net charge (Table I). There is one cationic tetraphenylporphyrin that carries a permanent $4+$ charge (tetrakis $(p-$ trimethylammoniopheny 1 ) porphine ${ }^{4+}, \mathrm{H}_{2} \mathrm{TMAPP}^{4+}$, , but most substituted tetraphenylporphyrins are either charged or neutral depending upon the $\mathrm{pH}$ of the solution.

It is obvious that if the functional group can undergo acid-base reactions, then the tetraphenylporphyrin's aqueous solubility is a function of $\mathrm{pH}$; tetrakis( $p$-nitrophenyl)porphine $\left(\mathrm{H}_{2} \mathrm{TNPP}^{0}\right)$, tetrakis $(p-$ 
aminophenyl)porphine $\left(\mathrm{H}_{2} \mathrm{TAPP}\right)$, and tetrakis(4-pyridyl)porphine $\left(\mathrm{H}_{2} \mathrm{TPy}^{\mathrm{T}}\right.$ ) are water soluble only within the $\mathrm{pH}$ range in which their functional groups are protonated (i.e. $\mathrm{H}_{2} \mathrm{TAPP}^{4+}, \mathrm{H}_{2} \mathrm{TPy}^{4+}, \mathrm{H}_{2} \mathrm{TNPP}^{4+}$ ).

TABLE I

PORPHYRINS USED IN THIS STUDY

\begin{tabular}{|l|l|l||}
\hline \multicolumn{1}{|c|}{$\begin{array}{l}\text { Chemical structure of the Substituted Tetraphenylporphyrins } \\
\mathrm{X}=\text { Functional Group }\end{array}$} \\
\hline
\end{tabular}


Anionic tetraphenylporphyrins contain either sulfonate $\left(\mathrm{H}_{2} \mathrm{TS}_{3} \mathrm{PP}^{3-}\right.$. $\left.\mathrm{H}_{2} \mathrm{TSPP}^{4-}\right)$, or carboxylate $\left(\mathrm{H}_{2} \mathrm{TCPP}^{4-}\right)$ groups. $\mathrm{H}_{2} \mathrm{TSPP}^{4-}$ is the most watersoluble, since its highest $\mathrm{pk}_{\mathrm{a}}$ is 4.8 (49); thus it exists in the tetraanionic form above $\mathrm{pH} \approx 6$. In addition, the sulfonate group is more polar than the carboxylate group increasing its relative solubility. Pasternack and coworkers (83-85) have shown that the aqueous solubilities of $\mathrm{H}_{2} \mathrm{TCPP}^{4-}$ and $\mathrm{H}_{2} \mathrm{TS}_{3} \mathrm{PP}^{3-}$ are similar at pH values close to neutrality. Iiterature values for the $\mathrm{pK}_{a}$ 's of $\mathrm{H}_{2} \mathrm{TCPP}^{4-}$ vary $(67,83-86,91)$; however, in our lab (work done by Dr. Ray Bard) we found the highest $\mathrm{pk}_{\mathrm{a}}$ to be between 6 and 7 . This gives the molecule a net negative charge between 3.5 and 4 near neutral pH values (the charge will be assigned as -4 , for simplicity, in aqueous solution at pH values $\geq 7$ ). The solubility of $\mathrm{H}_{2} \mathrm{TCPP}^{4-}$ should be sensitive to changes in $\mathrm{pH}$ near neutrality, which explains the difficulty in studying this porphyrin in aqueous solutions and the importance of using buffers to control the pH.

Pasternack and coworkers (83-86) studied the effect of two inorganic salts on the aqueous solubility of several tetraphenylporphyrins. They concluded that increased ionic strength decreased aqueous solubility (through tetraphenylporphyrin dimerization). The source of this effect was thought to be via coulombic shielding which would reduce coulombic repulsion of porphyrin molecules of like charge. These authors also found that the salt effect was induced by the cation and not the anion by studying a series of alkali metal salts. They varied the anion with no variation in the cation or the observed effect. By correlating loss of linearity in the Beer's law plots with porphyrin dimerization, they found that $\mathrm{H}_{2} \mathrm{TCPP}^{4-}$ exists $\mathrm{i}_{\mathrm{il}}$ moñomer form only at concentrations at or below $2 \times 10^{-6} \mathrm{M}(92)$ at low ionic strength.

Increased ionic strength narrowed the linear region of the Beer's law plots. This was attributed to salt-induced porphyrin dimerization. These conclusions were drawn from observations of the loss in the 
absorptivity at the soret band (the $s_{0} \rightarrow s_{2}$ electronic transition, at $414.2 \mathrm{~nm}, \epsilon \approx 500,000 \mathrm{M}^{-1} \mathrm{~cm}^{-1}$ ). This important and useful work was performed nearly twenty years ago before the spectrum of the dimer was known (69).

As was mentioned previously, porphyrins dimerization may be faceto-face, edge-to-edge, or face-to-face through a $\mu$-oxo linkage. Unfortunately, much of the literature concerning dimer spectroscopy appears to be contradictory. For example, a few studies correlate a red-shifted soret band $(37,38,67)$ with porphyrin dimerization or aggregation, while some associate dimerization with a weakened, unshifted soret $(83-85,89)$. Still others correlate a blue-shifted, weakened soret $(61,69,70$,$) with the same phenomenon. In many instances$ the experienced porphyrin chemist is able to understand the apparent contradictions by correlating the spectral change with an anticipated dimer structure. For example, $\mu$-oxo dimers yield spectra which are redshifted and weakened relative to the monomer spectrum (70). In contrast, face-to-face dimers which do not have a $\mu$-oxo linkage yield spectra which are blue-shifted and weakened $(69,70)$. The author has observed that the cationic porphyrin $\mathrm{H}_{2} \mathrm{TAPP}^{4+}$ yields red-shifted dimers which may correlate with an edge-to-edge structure. A porphyrin chemist is usually able to interpret the published data without too much confusion by a careful reading of the methods and results sections. The author has implied in the previous paragraph that a specific spectral change can often be associated with a unique dimer structure. To this date one type of spectral change has not been carefully addressed. A loss in the absorbance of the porphyrin monomer, which is not accompanied by a red or blue shift, in response to increased salt or porphyrin concentration is attributed throughout the literature to porphyrin dimerization. The author will show that this effect may not always be correlated with dimerization. Evidence will be presented which shows that the absorptivity is a function of the salt 
concentration. This explains why porphyrin absorptivities determined in individual laboratories vary if they are determined under different experimental conditions.

Unique to this work is the interpretation that the spectral changes induced by low salt concentrations are not due to porphyrin dimerization. First let me qualify this statement by emphasizing that this effect is exclusive to inorganic cations. Second it should be mentioned that at higher inorganic cation concentrations a blue-shifted porphyrin spectrum is observed which I do correlate with porphyrin dimerization. Third, the effect of organic cations may be fundamentally different from that observed with inorganic cations. The reader will find this effect investigated in chapter IV.

Pasternack and coworkers did not observe a significant blue shift in the spectrum they attributed to the porphyrin dimer. In addition, the author found that much higher salt concentrations ( $3 \mathrm{M}$ ) than those used in this earlier work $(0.1 \mathrm{M}, 84)$ were required to obtain the expected blue shift. The evidence presented in this study does not support the dimerization model used to explain the loss in porphyrin absorptivity as a function of increased low (mM) alkali metal cation concentration. Moreover, it appears that the cation complexation cannot be used to explain the loss in absorptivity as Gouterman and coworkers $(28,121)$ have found no evidence to support alkali metal ion complexation by porphyrins in aqueous solution (at neutral pH values). Thus, by a process of elimination, I correlate the loss in porphyrin absorptivity as a function of increasing alkali metal cation concentration (in the $\mathrm{mM}$ cation concentration region) to a cation-induced spectral line shape change. This is a novel and untested interpretation.

The loss in linearity in the Beer's law plots may or may not be explained by dimerization. The author has assumed only that the linear region of the Beer's law plots corresponds to the existence of porphyrin monomer in solution without addressing the question of how deviation 
from the Beer's law plots should be interpreted. It should become apparent in the following chapters that although this may be an interesting and important question, it did not need be addressed to accomplish the goals of this work.

\section{ENVIRONMENTAL SIGNIFICANCE}

As was mentioned in the previous section, tetraphenylporphyrins are utilized in such diverse applications as solar energy conversion, light-sensitized tumor therapy, and trace metal analysis of seawater. This has stimulated intense research activity with a concomitant explosion in the volume of porphyrin (and substituted tetraphenylporphyrin) literature.

During the last ten years the metallo-complexes, and to a lesser degree the free base tetraphenylporphyrins, have been investigated as the sensitizers in light-catalyzed electron transfer chemistry (39-58). In addition to problems with dimerization (or aggregation), tetraphenylporphyrins complex with many electron acceptors, impeding net electron transfer. In order for net charge transfer to occur; 1) the acceptor must approach the photoexcited sensitizer closely enough to enable electron transfer, and 2) the products must diffuse apart for charge separation to occur. If the sensitizer and acceptor have formed a stable association complex, then the electron is very rapidly transferred back to the oxidized porphyrin in a process called "back electron transfer". The kinetics of back electron transfer are faster than dissociation (48). Therefore, complexation between porphyrins and acceptors greatly lowers the efficiency of photoinduced charge separation.

Electrostatic $(48)$ and/or hydrophobic interactions $(52,53,55-58)$ between donor and acceptor molecules could be important adjustable parameters in optimizing charge separation. By adjusting the charge differential between the donor and acceptor it might be possible to 
balance the attractive and repulsive forces such that close approach would be possible without the formation of association complexes. A similar logic could be applied to the addition of bulky groups which would result in steric hindrance to association. Therefore, one approach to minimizing the problem of back electron transfer is to study the association chemistry of porphyrin/acceptor systems in which the acceptors have been modified to yield different charge and/or structural characteristics (46-58).

Viologens (Table II) are the most often used electron acceptors for a number of reasons $(40,41)$ : 1) their reduction potentials allow electron donation from photoexcited porphyrins; 2) the hydrophobic or polar characteristics can be modified by substituting the " $R$ " groups without significantly altering the reduction potentials; 3) viologens absorb in the ultraviolet $(\approx 250 \mathrm{~nm})$ thus the viologen and porphyrin spectra do not overlap in the region of interest $(385-450 \mathrm{~nm}) ; 4$ ) the reduced viologen is colored with one prominent absorption maximum at 602 $\mathrm{nm}$, thus its formation can be assayed at a wavelength where the porphyrin has minimal absorbance; 5 ) in the presence of suitable catalysts such as platinum or hydrogenase, the reduced viologen can donate electrons to $\mathrm{H}^{+}$, forming $\mathrm{H}_{2}$.

The study of $\mathrm{H}_{2} \mathrm{TCPP}^{4-}$ was chosen for two reasons: 1) the asymmetric membrane/artificial photosynthesis project in our laboratory utilizes this porphyrin (71), and 2) $\mathrm{H}_{2} \mathrm{TCPP}^{4-}$ /acceptor systems have not been studied. In our laboratory, preliminary work performed by the author and $\mathrm{Dr}$. Valerie Anderson, has shown that $\mathrm{H}_{2} \mathrm{TCPP}^{4-}$ mediates the photoreduction of both $\mathrm{MV}^{2+}$ and $\mathrm{PVS}^{\mathrm{O}}$. However, there is good evidence that the efficiency could be increased, since the small amount of dynamic quenching in these systems is greatly outweighed by static quenching: 1) ground-state $\mathrm{H}_{2} \mathrm{TCPP}^{4-} / \mathrm{PVS}^{\mathrm{O}}$ and $\mathrm{H}_{2} \mathrm{TCPP}^{4-} / \mathrm{MV}^{2+}$ complex formation is observed via visible spectroscopy, 2) the fluorescence quenching of $\mathrm{H}_{2} \mathrm{TCPP}^{4-}$ by either $\mathrm{MV}^{2+}$ or $\mathrm{PVS}^{0}$ does not obey a simple 
TABLE II

VIOLOGENS USED IN THIS STUDY

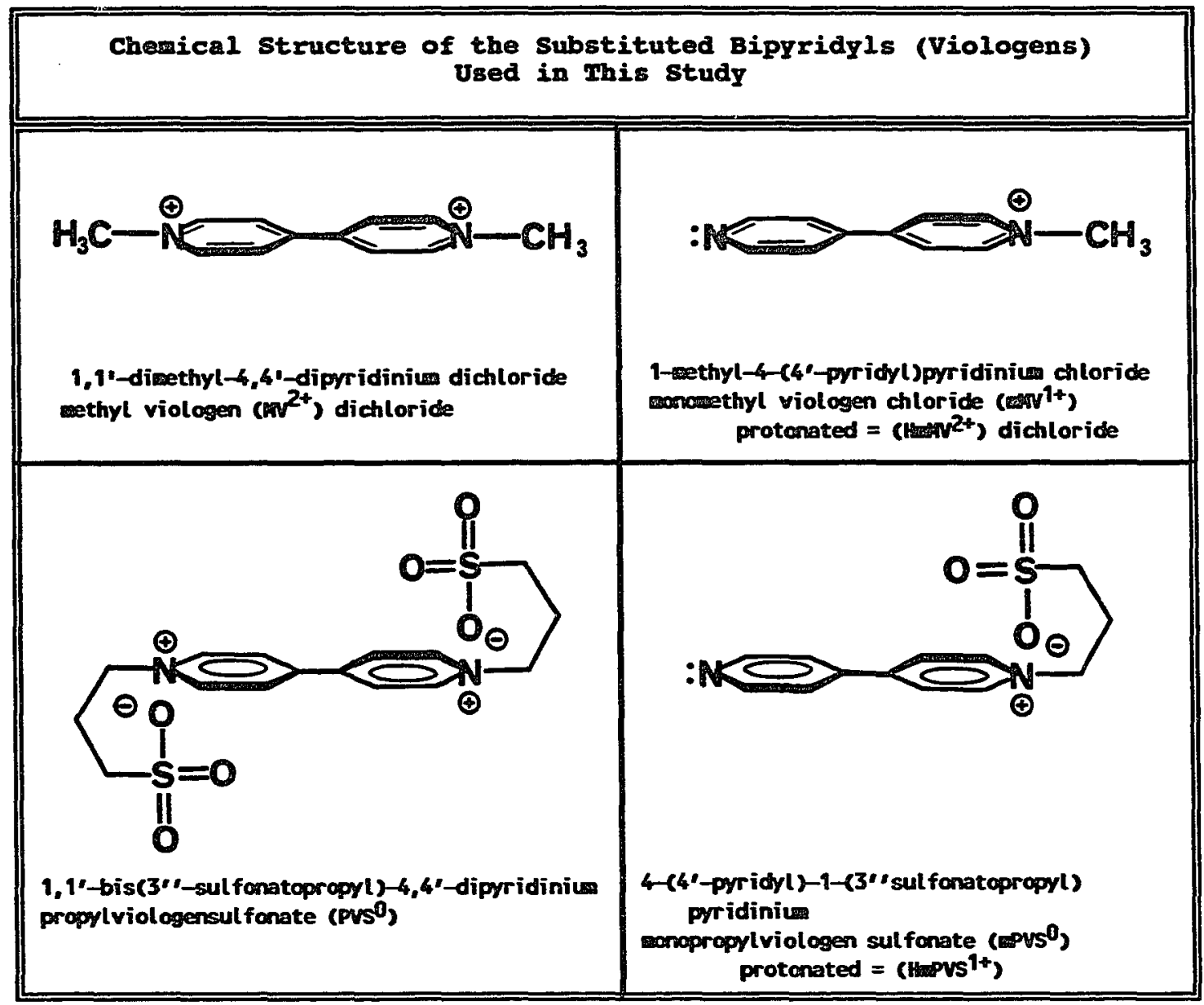

Stern-Volmer relationship (static quenching is observed), and 3) the $\mathrm{H}_{2} \mathrm{TCPP}^{4-}$ singlet lifetime is essentially independent of $\mathrm{PVS}^{\mathrm{O}}$ concentration (experiment performed by Dr. Valerie Anderson at CFKR, $U$. Texas, more evidence for static quenching\}.

Although many sophisticated techniques are employed in the study of tetraphenylporphyrins, these are almost always utilized in conjunction with absorbance spectroscopy. Specifically, published absorptivities are used to determine porphyrin concentrations; in addition, red or blue-shifts in wavelength maxima in response to added 
salts, detergents, or organic molecules are used to identify the porphyrin speciation in solution, and to quantify complexation or porphyrin dimerization.

From the discussion on porphyrin dimer spectroscopy it may be apparent that interpretation of changes in the porphyrin spectrum in response to salts or solvents is a complex subject. The author's concern is directed towards those scientists who are less experienced in the complexities of porphyrin chemistry, yet use porphyrins in important applied work. The last large review of the literature is contained in the series of volumes entitled "The Porphyrins" (6) which was written more than a decade ago and is at present seriously out of date on many subjects. In addition, this series of volumes is written for porphyrin chemists; thus the important methodology needed to successfully perform and interpret experiments is either absent or too defuse to be useful to scientists in applied fields. The author feels that a concise guide to experimental methodology and current interpretation of spectral data, for the specific audience of the applied scientist, is long overdue. The author hopes that this work will be useful to the applied scientist. It was initially intended that this work would involve a rather modest addition to the literature, in the form of quantification of a specific anionic free base tetraphenylporphyrin's ( $\mathrm{H}_{2} \mathrm{TCPP}^{4-}$ ) complexation by two viologens (PVS ${ }^{0}, M^{2+}$ ) with different properties. However, it rapidly became obvious that to interpret our spectroscopic data basic experiments needed to be performed. What has emerged is that these control experiments may be more significant than the porphyrin/viologen complexation study they were expected to clarify, especially in the area of the effect of salts on the porphyrin spectrum and speciation in solution.

Although determination of porphyrin/viologen association constants would be helpful to the specific application of $\mathrm{H}_{2} \mathrm{TCPP}^{4-} / \mathrm{viologen}$ systems in the field of solar energy conversion, a more fundamental 
contribution to the understanding of free base tetraphenylporphyrin solution behavior, with special emphasis on distinguishing spectral shifts due to porphyrin dimerization, from shifts due to other phenomena, would have broader application to the entire field of porphyrin research.

To conclude, we feel that this study is environmentally significant because porphyrins are of central importance in biological systems, and because their study is aimed at understanding and perhaps solving problems in a wide variety of applications, such as solar energy conversion.

\section{CONIRIBUTIONS OF THIS STUDY}

Several other studies of tetraphenylporphyrin complexation by viologens have utilized absorbance spectroscopy data to quantify the association constants $(44,51,46,47,52,55,57)$. A review of this literature can be found in chapter $V$. The author believes that the major contributions of this work to the porphyrin literature will be: a) to define experimental conditions under which these studies can be successful and b) to present a method for data analysis which is appropriate to the complexity of the problem.

The author will show that the titration of $\mathrm{H}_{2} \mathrm{TCPP}^{4-}$ with either $\mathrm{MV}^{2+}$ or $\mathrm{PVS}^{0}$ results in the formation of both $1: 1$ and $1: 2 \mathrm{H}_{2} \mathrm{TCPP}^{4-}$ :viologen complexes in contrast to the simple complexation reported by other authors for analogous systems $\left(\mathrm{H}_{2} \mathrm{TSPP}^{4-}: \mathrm{MV}^{2+}(46)\right.$ and $\mathrm{H}_{2} \mathrm{TS}_{3} \mathrm{PP}^{3-}: \mathrm{MV}^{2+}$ (52)). I encountered an unexpected difficulty when the determination of association constants was performed at constant ionic strength $(44,46,51,57)$. This will be illustrated by comparing results from experiments performed at constant ionic strength with those performed at variable ionic strength in which the buffer concentration was held constant. In addition, a novel method using principal component analysis (104-112), developed by the author and Harry E. Bell, to 
determine the number of absorbing species in solution and the absorptivity and solution concentration of each will be introduced. The author will refute data presented in two studies $(67,80)$ concerning the aqueous solubility of $\mathrm{H}_{2} \mathrm{TCPP}^{4-}$. I will also show that the accepted salt-induced dimerization model (correlating the change in porphyrin absorptivity with porphyrin dimerization as a function of millimolar concentrations of alkali metal ions) is insufficient to explain our spectral data; the alternative interpretation of a spectral line shape change is consistent with the data.

Finally, the author will present data which supports the conclusion of Firman et al. (69) that the spectrum of the $\mathrm{H}_{2} \mathrm{TCPP}^{4-}$ (or $\mathrm{H}_{2} \mathrm{TSPP}^{4-}$, dimer is weakened and blue-shifted relative to the porphyrin monomer in aqueous solution. I will further show that some organic ions induce anionic tetraphenylporphyrin dimerization and some do not; moreover, experimental evidence demonstrating that the data cannot be explained as a simple effect of the ionic strength will be presented. 
CHAPTER II

\section{PORPHYRIN SPECTROSCOPY: VISIBLE AND NMR}

\section{INTRODUCTION}

The choice of a suitable experimental method to study $\mathrm{H}_{2} \mathrm{TCPP}^{4-}$ : viologen association chemistry in solution at neutral pH values is constrained by several factors listed below.

1) The instrument must be sufficiently sensitive to allow detection of $\mathrm{H}_{2} \mathrm{TCPP}^{4-}$ within the concentration range where it is monomeric in aqueous buffered solutions, $i . e$. solutions containing salts. In Chapter IV we will establish that the upper concentration limit is $2 \mu \mathrm{M}$ at $5 \mathrm{mM}$ phosphate, and a $\mathrm{pH}$ value of 7.0 .

2) The method must be able allow to detection of all $\mathrm{H}_{2} \mathrm{TCPP}^{4-}$ species in solution, both porphyrin monomer, porphyrin dimer, and association complexes of $\mathrm{H}_{2} \mathrm{TCPP}^{4-}$ with viologen. Optimally, each species should be resolvable by the method of choice.

3) In Chapter $\mathrm{V}$ we will establish that the titration of $\mathrm{H}_{2} \mathrm{TCPP}^{4-}$ with $\mathrm{MV}^{2+}$ is not simple, as $\mathrm{H}_{2} \mathrm{TCPP}^{4-}: \mathrm{MV}^{2+}$ association complexes with stoichiometries of both $1: 1$ and $1: 2$ are observed. The titration of $\mathrm{H}_{2} \mathrm{TCPP}^{4-}$ with $\mathrm{PVS}^{0}$ is further complicated by the formation of porphyrin dimers at high viologen concentration in addition to the association complexes with stoichiometries of $1: 1$ and $1: 2$ ( $\left.\mathrm{H}_{2} \mathrm{TCPP}^{4-}: \mathrm{PVS}^{0}\right)$. Association constants are difficult to determine mathematically when multicomponent equilibria are observed (113-120). This is particularly true when the associations are weak (117), as is observed in porphyrin/viologen complexation. Thus, data uncertainty must be minimized in order to obtain meaningful values for the association constants (119). This requires measurements with high signal to noise 
ratios and minimal output correction.

Table III below summarizes the specific sensitivity, to $\mathrm{H}_{2} \mathrm{TCPP}^{4-}$, of three instrumental methods. This information shows why absorption spectroscopy (in the soret region) is best suited for our studies.

TABLE III

COMPARISON OF THREE INSTRUMIHMIAT MEMHODS

\begin{tabular}{|c|c|c|c|c|}
\hline \multirow[t]{2}{*}{$\begin{array}{l}\text { INSTRUMENTAL } \\
\text { METHOD }\end{array}$} & \multicolumn{2}{|c|}{$\begin{array}{l}\text { OPTIMAL CONCENTRATION } \\
\text { RANGE }\end{array}$} & \multirow[t]{2}{*}{$\begin{array}{l}\text { SIGNAL-TO- } \\
\text { NOISE RATIO }\end{array}$} & \multirow[t]{2}{*}{ COMMENTS } \\
\hline & Soret Band & Q Bands & & \\
\hline Fluorescence & $.01-0.1 \mu \mathrm{\mu M}$ & $.05-1.0 \mu \mathrm{M}$ & poor-fair & $\begin{array}{l}\text { 1) An indirect method. } \\
\text { 2) Detects porphyrin monomer and dimer } \\
\text { fluorescence. Porphyrin: viologen complexes are } \\
\text { quenched, thus are fluorescence silent. } \\
\text { 3) Requires correction for self-absorbance, } \\
\text { source intensity variations, and source output. } \\
\text { 4) High photon flux can cause sample degradation } \\
\text { in presence of } \mathrm{O}_{2} \text {. }\end{array}$ \\
\hline Absorbance & $1.0-2.0 \mu \mathrm{H}$ & $10-20 \mu \mathrm{N}$ & excellent & $\begin{array}{l}\text { 1) A direct method. } \\
\text { 2) Requires no correction. } \\
\text { 3) Loh photon flux minimizes danger of sample } \\
\text { degradation. } \\
\text { 4) Detects all porphyrin species }\end{array}$ \\
\hline $1_{H}$ MMR & $1-100 \mathrm{~mm}$ & $1-100 \mathrm{~mm}$ & fair-good & $\begin{array}{l}\text { 1) A direct method. } \\
\text { 2) Requires no correction. } \\
\text { 3) Ho problems with sample degradation. } \\
\text { 4) Gives excellent information on complexation } \\
\text { and/or dimerization. }\end{array}$ \\
\hline
\end{tabular}

Fluorescence spectroscopy could be useful to detect the loss of monomer, as porphyrin is complexed with viologen, during the progress of the titration of $\mathrm{H}_{2} \mathrm{TCPP}^{4-}$ with viologen; however, numerous technical problems have increased the uncertainty of the fluorescence data which were collected. Although fluorescence data are not presented here, this instrumental method may be utilized in future work.

As can be seen in Table III, ${ }^{1} H$ NMR is excluded on the grounds of 
sensitivity. Yet this method was very useful in some limited aspects of this study and will be discussed in the second section of this chapter.

\section{VISIBLE ABSORBANCE SPECIROSCOPY}

The data presented in this study were collected primarily from the visible region of the free base tetraphenylporphyrin spectrum between 385 and $500 \mathrm{~nm}$. Since all subsequent chapters display spectral data, a discussion of porphyrin spectroscopy is presented at this point. Spectra of metalloporphyrins and the bands from 350 to $700 \mathrm{~nm}$ are included to clarify the discussion.

In the visible and ultraviolet region of the electromagnetic spectrum molecules undergo electronic transitions. The porphyrin absorbance within this energy range can be explained by the porphine core; this electronic "heart" is responsible for the characteristic porphyrin-type spectra (28), which are then "perturbed" to a greater or lesser extent by various chemical modifications to the basic structure.

The current explanation of the porphyrin spectra is as follows: modification of the ring periphery via substitution shifts the wavelength and affects the absorptivity of the observed absorption maxima; however, any modification which results in nonequivalent pyrrole imino nitrogens lowers the symmetry, which changes the number of observed bands.

\section{D $_{4 h}$ Symmetry}

Porphyrin chemistry is best understood when the porphyrin molecule is viewed as a tetradentate chelator. The metal binding site is within the central cavity, as the four pyrrole nitrogens are the metal ligands, providing a square planar geometry as shown in Figure 1. This site is particularly favored by small, hard, metal ions with a preference for square planar or octahedral ligand fields, although most metal ions complex with porphyrins regardless of size or preferred geometry. When this site is occupied by a metal ion, the four nitrogens are equivalent 
and the molecule has a fourfold axis of symmetry $\left(D_{4 h}\right)$.

The two resonance structures of the $\pi$ electron system in a metalloporphyrin are represented in part " $\mathrm{A}$ " of Figure 1 . The presence of two equivalent conjugation paths (stippled regions) implies exact fourfold symmetry (30). Data from $x$-ray studies (30) show the outer carbon-carbon bonds to be essentially ethylenic; this supports

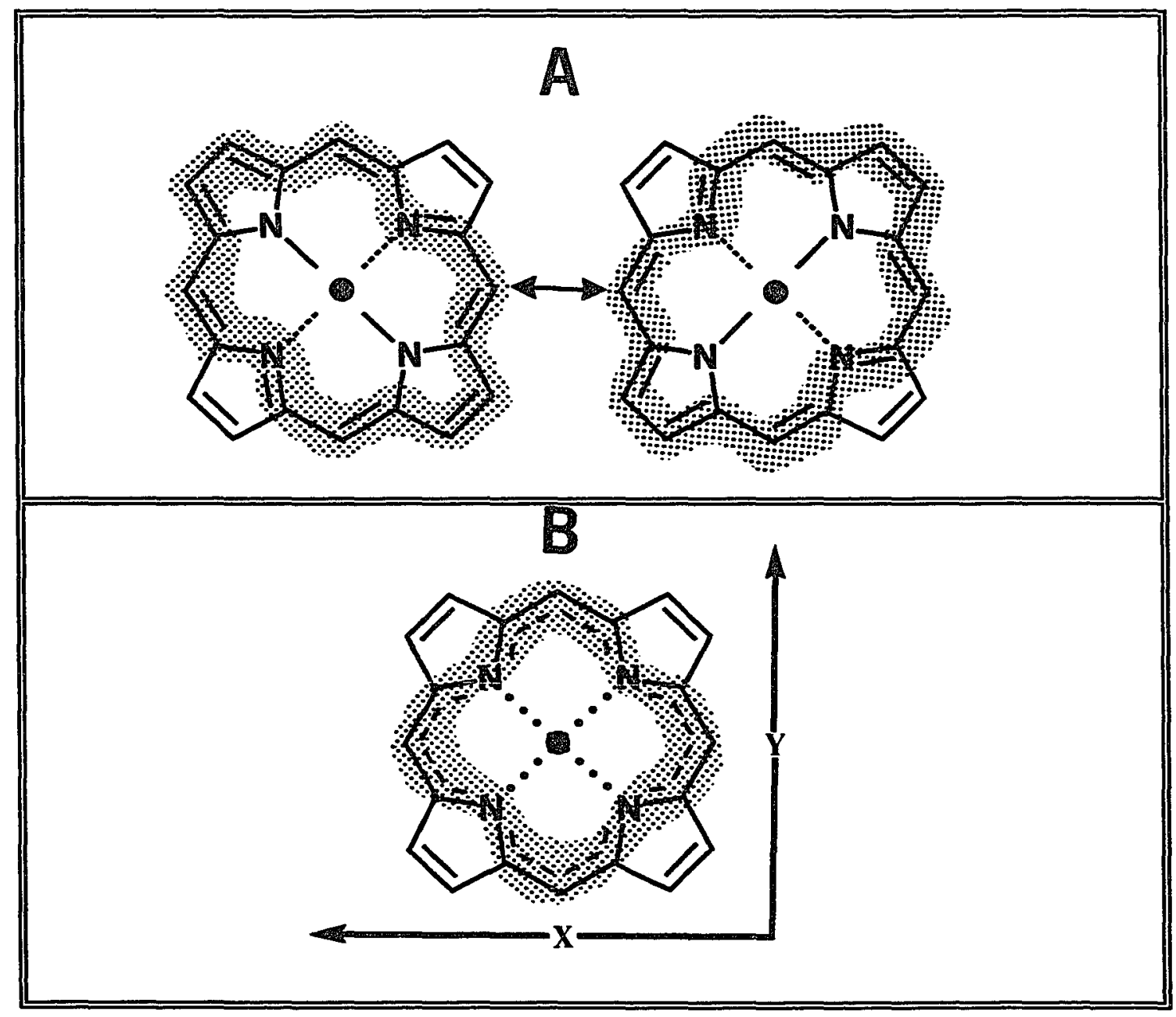

Figure 1. Resonance of the porphine macrocycle in $\mathbf{D}_{4 h}$ symmetry. The central "•" indicates any mechanism which results in equivalent pyrrole nitrogens. Part "A" shows the two resonance structures; the relevant conjugated region is shaded (保). Notice that in each resonance structure one pair of B-pyrrole ethylenic bonds is excluded, making these bonds less important in the resultant resonance structure shown in part "B". The dashed (--) and shaded (E) portions indicate the relevant resultant resonance structure. 
molecular orbital calculations $(28,30)$ which conclude that the relevant $\pi$ system is the 16-membered inner ring shown in part "B" of Figure 1 above. The electron book-keeping for the inner ring system is as follows: there are 16 atoms (12 carbons and 4 nitrogens, all $\mathrm{sp}^{2}$ ), one $\mathrm{p}_{\pi}$ electron from each carbon atom, one $\mathrm{p}_{\pi}$ electron from two imino nitrogens, two $\mathrm{p}_{\pi}$ electrons from alternate imino nitrogens for a total of $18 \pi$ electrons. An $18 \pi$ electron conjugated system follows the Hückel criterion for an aromatic $4 n+2$ system, where $n=4$.

When the "metal" is hydrogen, the symmetry depends upon the number of hydrogens bound. In strongly acidic solutions four hydrogens may be bound yielding equivalent nitrogens. An illustration of the chemical structure of $\mathrm{H}_{4} \mathrm{TAPP}^{6+}$ is shown in Figure 2 below.

In strongly basic solutions the imino nitrogens are deprotonated and the molecule also shows spectra typical of $D_{4 h}$ symmetry. However, it has been shown $(28,32)$ that the porphyrin is not metal-free, eg. TCPP6- TAPP $^{2-}$. Instead, the porphyrin is complexed to the cation under these conditions (even if the cation is a group I metal ion). In $D_{4 h}$ symmetry four bands are observed between 350 and $700 \mathrm{~nm}$ : the $s_{0} \rightarrow s_{1}$ electronic transition called " $Q$ " band (all bands in the wavelength region from 450-700 $\mathrm{nm}$ are also termed "Q"), and the $S_{0} \rightarrow S_{2}$ electronic transition called the "B" or "Soret" band. Each transition shows an additional higher energy band $(28,30,31)$ which is assigned as one mode of vibrational energy $(1 \leftarrow 0)$. However, as will be described later, this assignment is uncertain for the high energy shoulder on the Soret band.

In Figure 2 an example of the spectral patterns observed in $D_{4 h}$ symmetry is illustrated. Typically, there are two $Q$ bands between 500 and $650 \mathrm{~nm}$ plus two bands in the near UV (350-430 nm). All porphyrins with $\mathrm{D}_{4 h}$ symmetry show this spectral pattern; no exceptions have been reported to the best of the author's knowledge. 


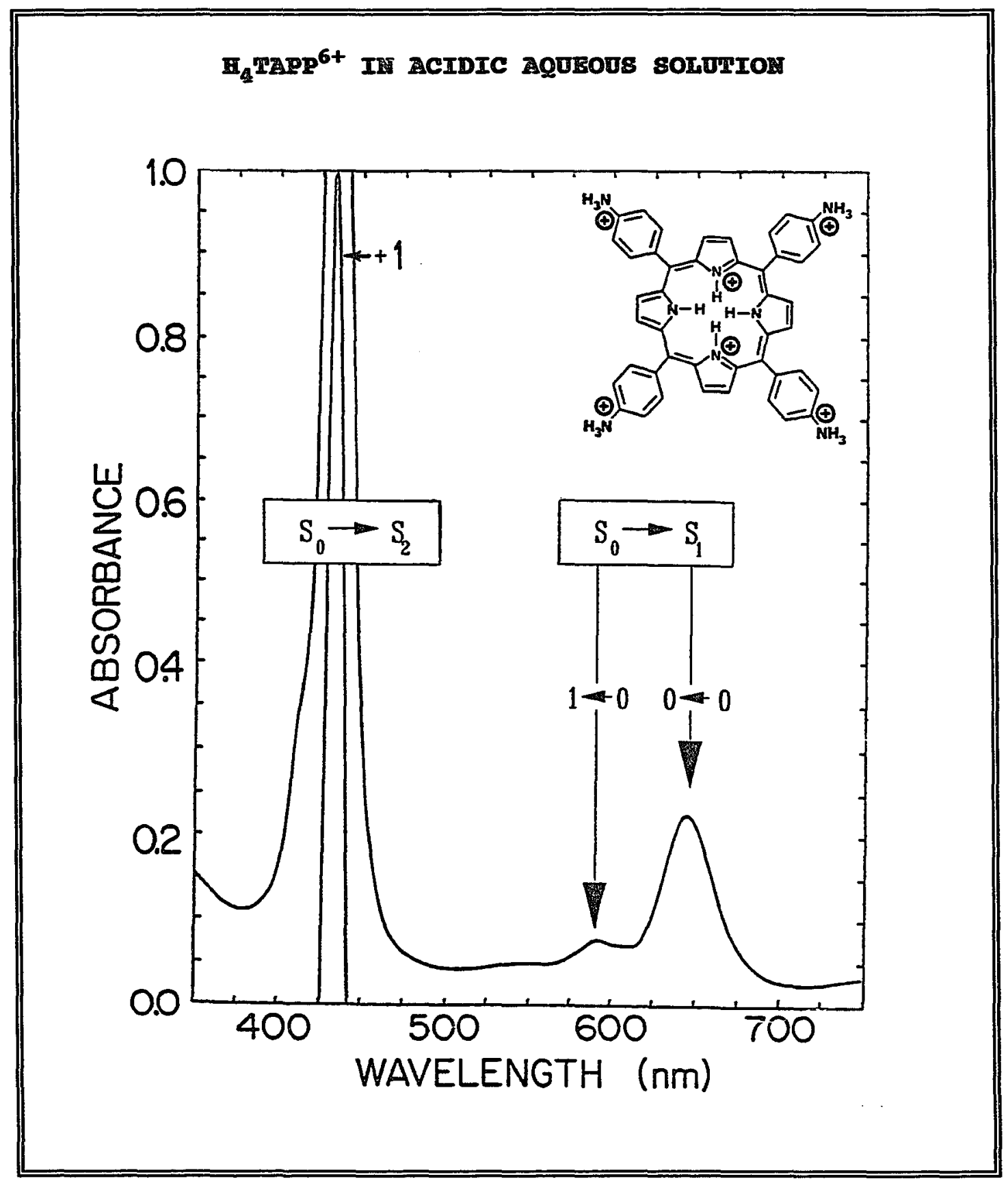

Fiqure 2. Spectrum of $\mathrm{H}_{4} \mathrm{TAPP}^{6+}$ in acidic aqueous solution ( $\mathrm{pH} \approx 0.7$ ) showing the $0 \leftrightarrow-0$ and $1 \leftarrow 0$ components of the $\mathrm{S}_{0} \rightarrow \mathrm{S}_{1}$ " $Q$ " transitions. The highest energy transition in this region is unassigned. The $S_{0} \rightarrow S_{2}$ electronic transition or "Soret" band is also shown; the high energy shoulder on the soret is usually assigned as the $1 \leftarrow 0$ mode. 
D $_{2 h}$ Symmetry

The "free base" porphyrin, where two hydrogens are bound to alternate nitrogens is shown in Figure 3; since the nitrogen atoms are

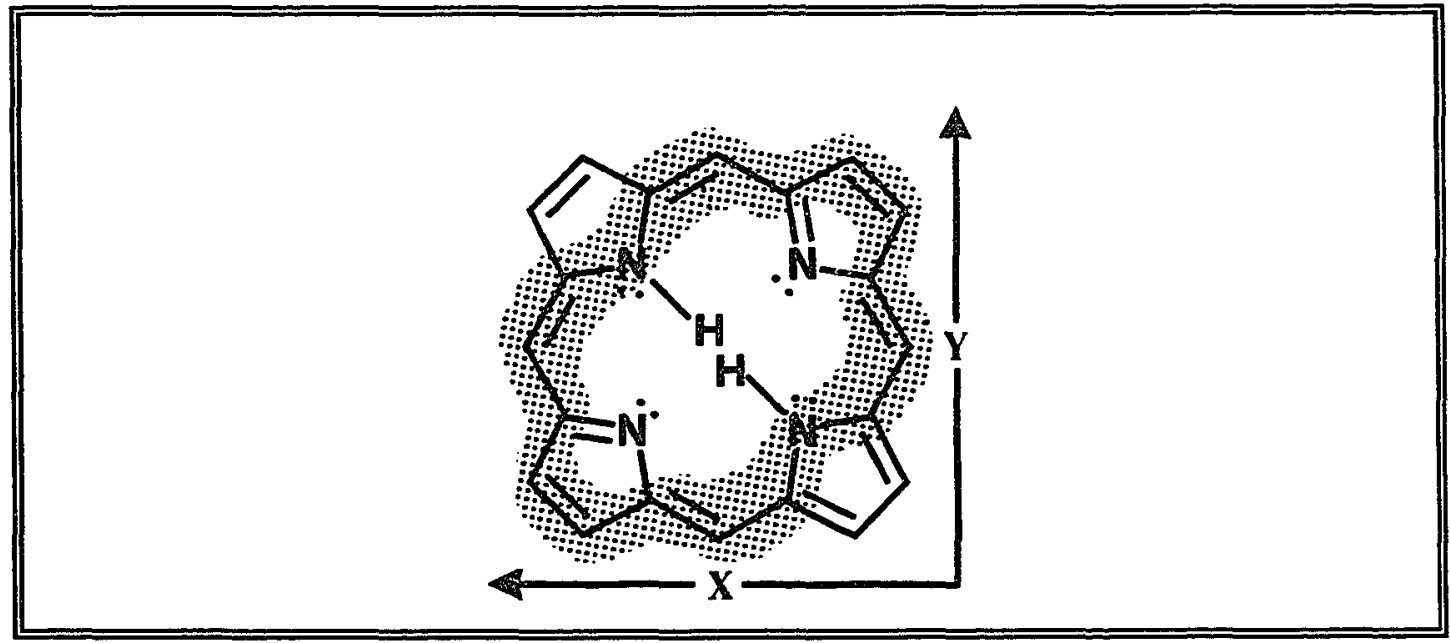

Figure 3. Resonance of the porphine macrocycle in $D_{2 h}$ symmetry. Notice that the two protons bound to alternate central nitrogens results in a rectangular resonance path. The relevant conjugated region is shaded (:

no longer equivalent, the symmetry is reduced from $D_{4 h}$ to $D_{2 h}$. The 16membered $18 \pi$ electron system in $D_{4 h}$ symmetry becomes an 18 -membered polyene with $18 \pi$ electrons in $\mathrm{D}_{2 \mathrm{~h}}$ symmetry. Notice that the two hydrogens fix the principal resonance structure into a rectangular path. This results in nonequivalent $x$ and $y$ axes which helps to explain the difference between the spectra of $D_{4 h}$ metalloporphyrins (or the acidic "dication" e.g. $\mathrm{H}_{4} \mathrm{TCPP}^{2+}, \mathrm{H}_{4} \mathrm{TAPP}^{6+}$, and the $\mathrm{D}_{2 h}$ free base porphyrins, e.g. $\mathrm{H}_{2} \mathrm{TCPP}^{4-}, \mathrm{H}_{2} \mathrm{TAPP}^{0}$.

For the free base porphyrins, the $Q$ bands are strongly split due to nonequivalent absorbance along the $x$ versus $y$ axes $y$ ielding four bands between 450 and $700 \mathrm{~nm}$, as shown in Figure 4, rather than two bands as illustrated in Figure 2. Since the $x$ and $y$ axes are not equivalent, the energy of the absorbance yielding the two $Q$ bands differs depending upon whether the absorbance occurs along the $x$ or $y$ 


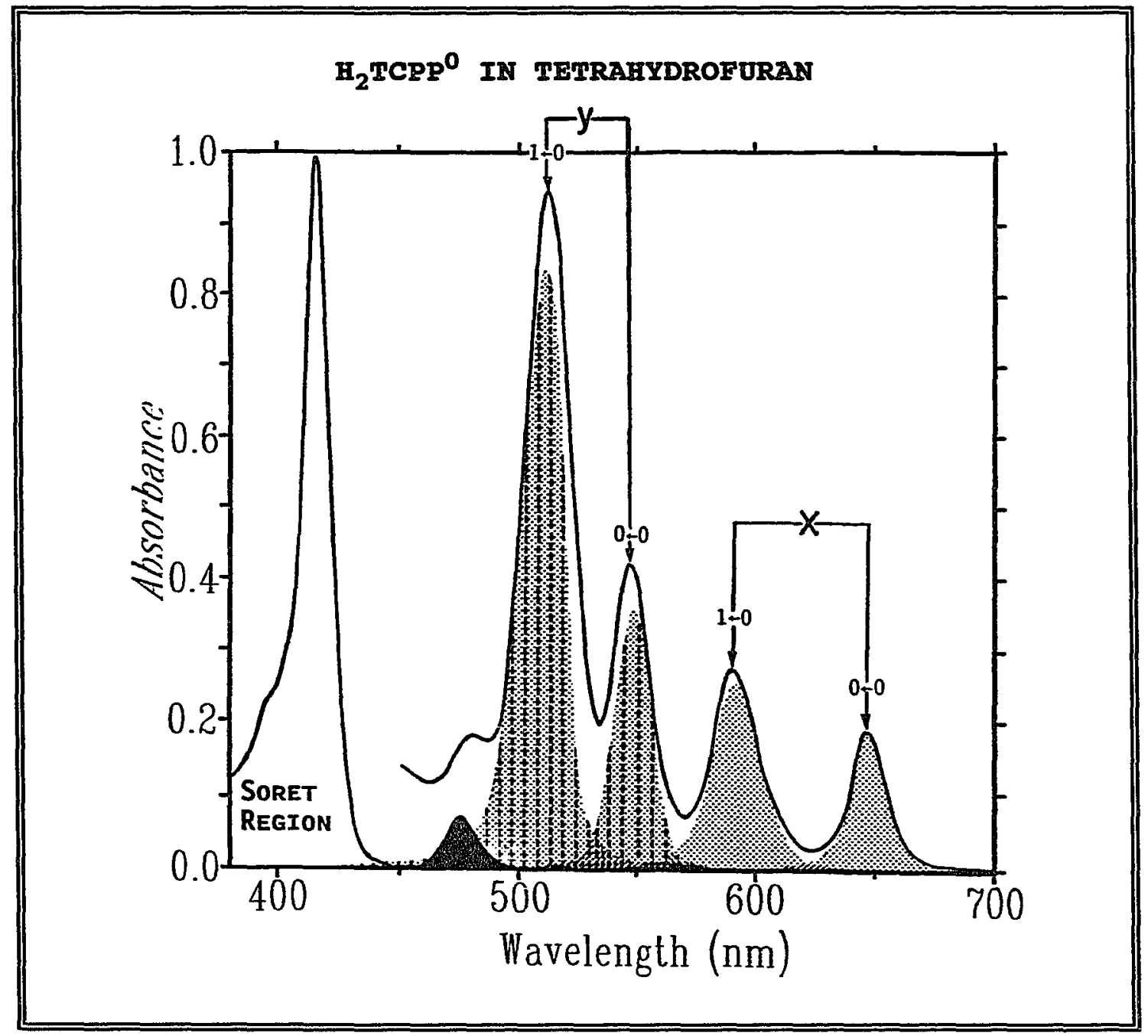

\begin{abstract}
Fiqure 4. Spectrum of $\mathrm{H}_{2} \mathrm{TCPP}^{0}$ in tetrahydrofuran. The $\mathrm{Z}$ and $Y$ splitting of the $Q$ band region is shown. The $Z$ components are shaded (位), the $Y$ components are shaded and striped; peak areas and shapes are approximate. The black peak is unassigned. $\left[\mathrm{H}_{2} \mathrm{TCPP}^{0}\right]$ in soret region is $\approx 10 \mathrm{x}$ less than that in the $Q$ band region.
\end{abstract}

orientation. One can visualize that Figure 4 arises as a composite of the spectrum of molecules oriented with their $x$ axes parallel, plus the spectrum of molecules oriented with thier $y$ axes parallel, to the plane of the oncoming electric component of the electromagnetic wave.

The MCD spectra (31) of $\mathrm{H}_{2}$ TPP revealed that the soret band is also split into $x$ and $y$ components; however, since these components are 
separated by less than a bandwidth, they are not resolved in the absorbance spectrum and appear as a single peak. Figure 5 shows the spectrum with the peaks resolved mathematically. This was accomplished by decomposing the spectrum of $\mathrm{H}_{2} \mathrm{TCPP}^{4-}$, plotted on an energy scale, into a linear combination of 3 Gaussians plus án energy baseline. Unlike the high energy shoulder, it was found that the soret band could not be fit by a single Gaussian but was readily fit by two, which is

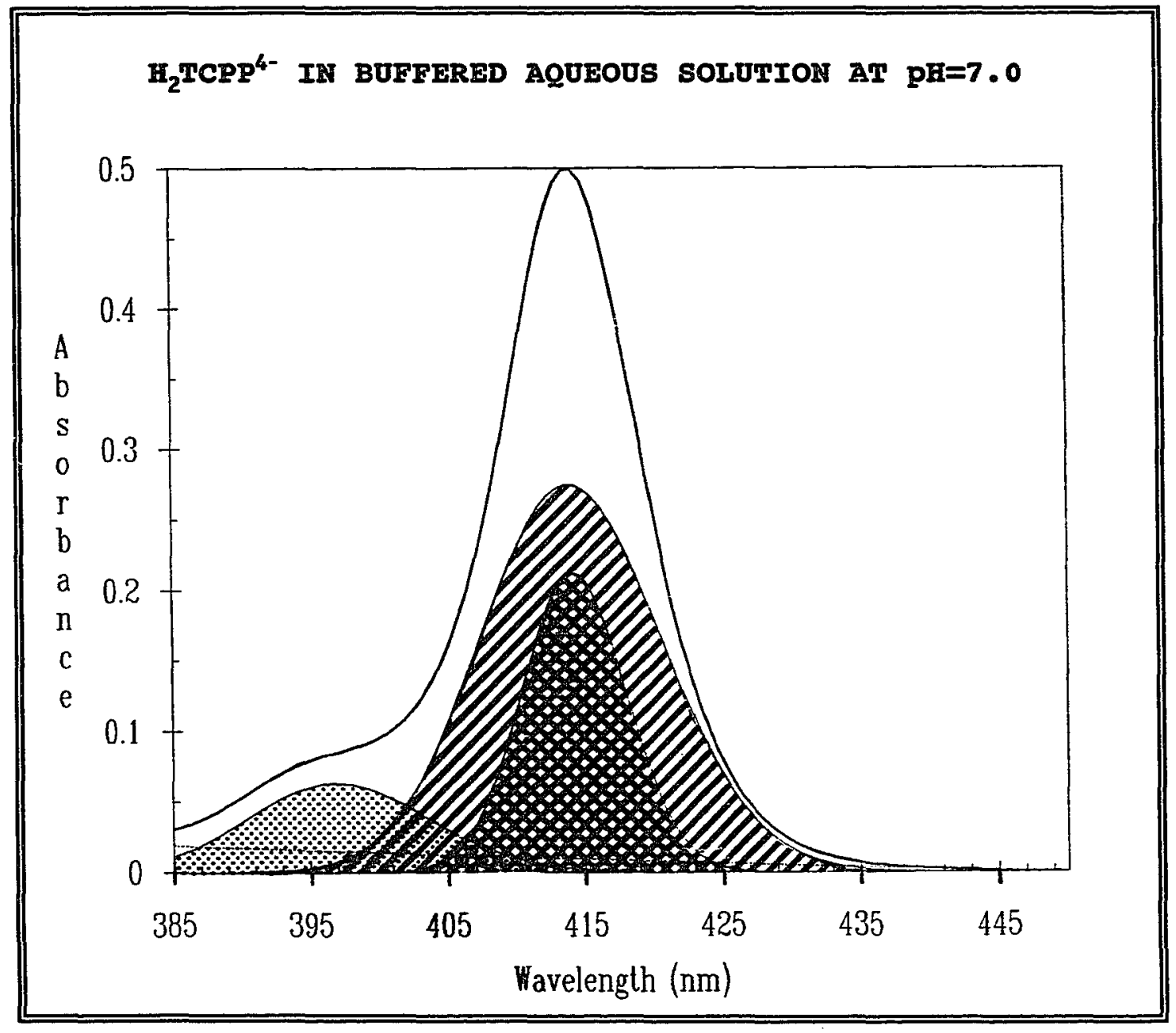

Figure 5. Spectrum of $1 \mu \mathrm{M} \mathrm{E}_{2} \mathrm{TCPP}^{4-}$ in aqueous solution buffered to $\mathrm{pH}=7$ with $5 \mathrm{mM}$ phosphate. The $\mathrm{X}$ and $Y$ components of the $s_{0} \rightarrow s_{2}$ electronic transition, or soret band (striped plus crosshatched bands) are shown. The identity of the $X$ and $Y(0 \leftarrow-0)$ components are not known. The high energy shoulder (保) is usually assigned as one vibrational mode $(1 \leftarrow 0)$. 
consistent with the MCD data. The shapes of the bands do not appear Gaussian in Figure 5, as they are plotted on a wavelength rather than energy scale.

The identification of the $x$ and $y$ bands in the porphyrin spectra comes from interpretation of magnetic circular dichroism (MCD) data $(28,30,31)$. To the best of the author's knowledge, neither $\mathrm{H}_{2} \mathrm{TCPP}^{4-}$, $\mathrm{H}_{2} \mathrm{TSPP}^{4-}$, nor $\mathrm{H}_{2} \mathrm{TAPP}^{0}$ have been studied by MCD. Nevertheless, identification of the $\mathrm{Q}$ band region for $\mathrm{H}_{2} \mathrm{TCPP}^{4-}$ and $\mathrm{H}_{2} \mathrm{TSPP}^{4-}$ is possible, as the absorbance spectral pattern for these two porphyrins is identical to the parent, $\mathrm{H}_{2}$ TPP, whose bands have been assigned using MCD (31). Therefore, the assignments shown in Figures 4 and 5 are based upon the assignments for $\mathrm{H}_{2} \mathrm{TPP}$.

\section{The Two Unassiqned Bands}

Although much attention has been given to understanding the porphyrin spectrum, two bands remain unassigned: the band at approximately $475 \mathrm{~nm}$ which is shown in black in Figure 4, and the high energy shoulder on the soret band at approximately $400 \mathrm{~nm}$, which is shaded (㘗) in Figure 5. It is thought that all bands in the visible and near UV are $\pi \rightarrow \pi *$ transitions; however, the lowest energy $n \rightarrow \pi *$ transition is still controversial $(28,30,31)$.

The evidence that implicates the $400 \mathrm{~nm}$ band as an $\mathrm{n} \rightarrow \pi *$ transition arises from MCD data obtained for $\mathrm{H}_{2}$ TPP $(28,31)$. Primarily, it appears that the $400 \mathrm{~nm}$ band is not split, and gives a Y orientation (the $475 \mathrm{~nm}$ band gives an $\mathrm{x}$ orientation). One (of the many) molecular orbital calculations (28) predicts an $n \rightarrow \pi^{*}$ transition for the $400 \mathrm{~nm}$ band which is consistent with the MCD results (31), and our observation that this band can be fit by a single Gaussian. Nevertheless, it has become widely accepted that this band is the first vibrational component of the $s_{0} \rightarrow s_{2}$ electronic transition $(70,121)$.

Although this work does not address the issue of band assignments, observations of our data have aroused a curiosity concerning the 
assignment of the $400 \mathrm{~nm}$ band. It is interesting that the spectrum of the dimer has its maximum at $400 \mathrm{~nm}$. This is interpreted in the literature as the result of excitonic coupling $(70,122-125)$, where the $400 \mathrm{~nm}$ band is the allowed component of the $s_{0} \rightarrow s_{2}$ transition of the porphyrin dimer $(11,54)$. Another interpretation might be that the soret band is weakened and the $400 \mathrm{~nm}$ band is strengthened. This is a subject which is beyond the scope of this work, but deserves further attention.

\section{The Effect of Substituents, Solvents, and pH}

The Effect of solvents. The information and interpretations in previous sections of this chapter are based on widely accepted principles coupling porphyrin core symmetry with spectral patterns. since the biologically significant porphyrins are all chelated to metals, and since the metal species is critical to optimize the functional role of biological metalloporphyrins, it is not surprising that nearly all of the spectroscopic studies focus on the effect of metal or ligand substitution on spectral patterns. For example, the effect of solvent is viewed as the influence of axial ligation on the spectral pattern of metalloporphyrins.

In this section, we cross the frontier from data interpretation, by analogy to the literature, to speculative data analysis in areas that have not been addressed. One area which remains unexplored is the effect of functional groups not directly bonded to the porphine core and attached in a manner such that the symmetry $\left(D_{4 h}\right.$ or $\left.D_{2 h}\right)$ is not affected. The principles introduced in the second section of this chapter predict that this type of modification should not perturb the observed spectrum, i.e. the spectrum of the porphyrin modified as described above should resemble the parent unmodified porphyrin. For example, the addition of phenyl groups to the porphine core does not perturb the porphine spectral pattern. In addition, the presence of carboxylate or sulfonate groups in the phenyl para positions also does not change the spectral pattern. This section explores why the presence of amino groups in the 
phenyl para positions is an exception.

In this discussion we categorize $\mathrm{H}_{2} \mathrm{TCPP}^{4-}$ and $\mathrm{H}_{2} \mathrm{TSPP}^{4-}$ as "well behaved" and $\mathrm{H}_{2} \mathrm{TAPP}^{0}$ as "not well behaved". What is meant here by "well behaved" is that the substitution of functional groups in the phenyl para positions do not perturb the spectral pattern observed for the parent molecule, $\mathrm{H}_{2} \mathrm{TPP}$. We define two criteria which are met by $\mathrm{H}_{2} \mathrm{TCPP}^{0}$ as illustrated in Figures 4 and 6 , but not met by $\mathrm{H}_{2}$ TAPP $^{0}$ as illustrated in Figures 7 and 8 . These criteria are: 1) no perturbation in the tetraphenylporphyrin spectrum observed upon the addition of functional groups in the phenyl para positions and 2) a spectral pattern which is independent of solvent (although, as is to be expected, the wavelengths of the peak maxima are solvent dependent).

The spectrum of $\mathrm{H}_{2} \mathrm{TCPP}^{4-}$ in aqueous solutions (Chapters IV and V) near neutral $\mathrm{pH}$ values does not differ significantly from $\mathrm{H}_{2} \mathrm{TCPP}^{\mathrm{O}}$ in organic solvents (Figures 4 and 6 ). In addition, the spectral pattern for $\mathrm{H}_{2} \mathrm{TCPP}^{0}$ is not dependent upon the identity of the organic solvent as is observed in THF (Figure 4), and in chloroform and dimethylsulfoxide (Figure 6).

Whereas the spectra of $\mathrm{H}_{2} \mathrm{TPP}$ and $\mathrm{H}_{2} \mathrm{TCPP}^{0}$ are completely analogous, a very different situation is observed in comparing the spectra of $\mathrm{H}_{2} \mathrm{TPP}$ with $\mathrm{H}_{2}$ TAPP $^{0}$ (Figure 7). The substitution of amino groups in the phenyl para positions specifically affects the absorption pattern of the vibrational bands. This can be seen by contrasting the spectra of $\mathrm{H}_{2} \mathrm{TCPP}^{0}$ in THF (Figure 4) with the spectra of $\mathrm{H}_{2}$ TAPP $^{0}$ in THF (Figure 7 ). For $\mathrm{H}_{2} \mathrm{TCPP}^{0}$ the absorbance of the vibrational bands $(1 \leftarrow 0, \mathrm{X}$ and $\mathrm{Y}$; $\left.s_{0} \rightarrow s_{1}\right)$ is greater than the absorbance of the corresponding $0 \leftarrow 0$ electronic transition. This pattern is reversed for $\mathrm{H}_{2} \mathrm{TAPP}^{0}$, as the vibrational components $\left(1 \leftarrow 0, X\right.$ and $\left.Y_{;} S_{0} \rightarrow S_{1}\right)$ have a lower absorbance than the corresponding $0 \leftarrow 0$ electronic transition. In addition, the $\mathrm{H}_{2}$ TAPP $^{0}$ vibrational Q-bands are very sensitive to solvent (Figures 7 and 8), a situation not observed for $\mathrm{B}_{2} \mathrm{TCP}^{0}$. 

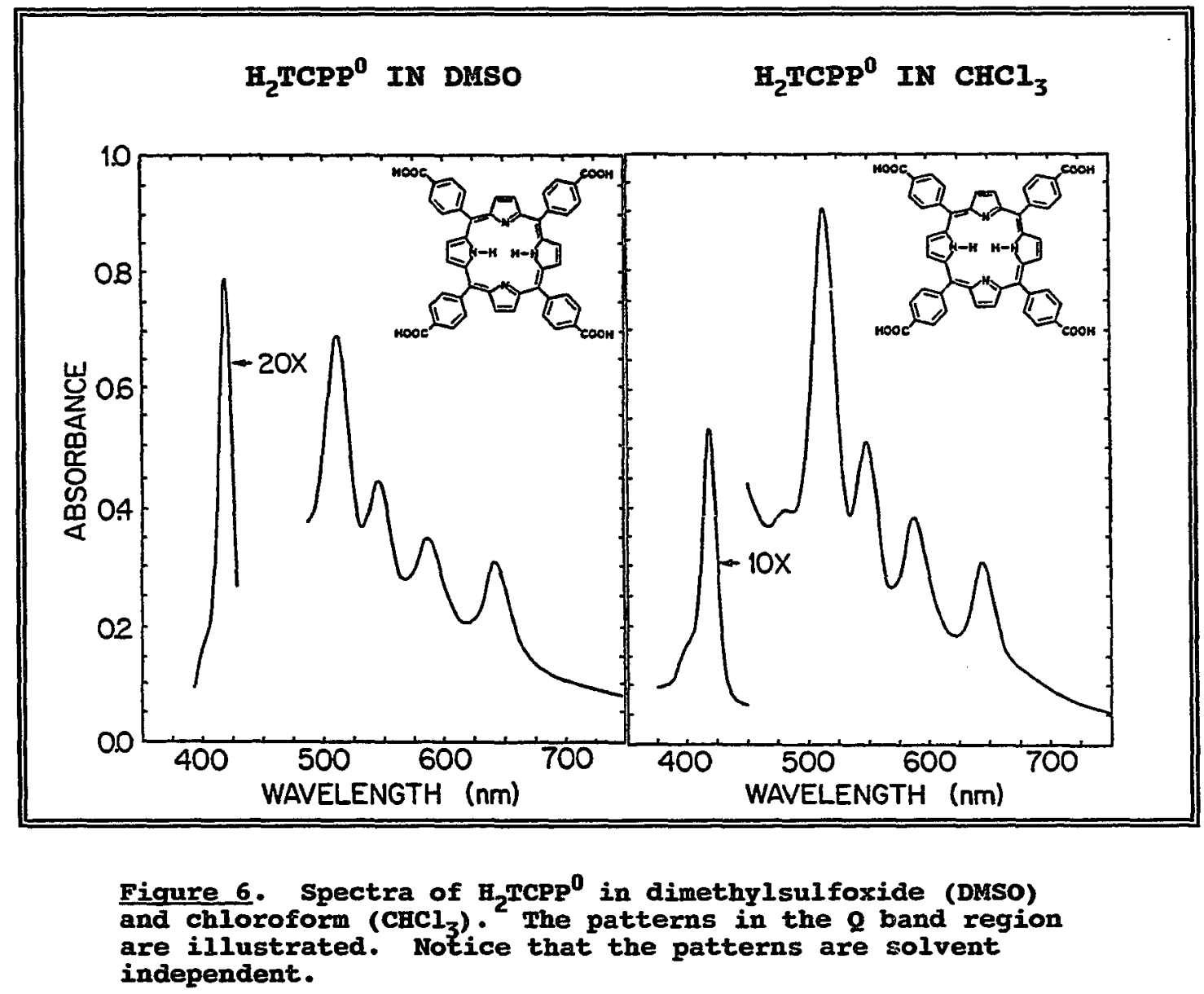

The difference between the spectra of $\mathrm{H}_{2} \mathrm{TCPP}^{0}$ and $\mathrm{H}_{2} \mathrm{TAPP}^{0}$ must be due to some characteristic present in the amino group and not present in the carboxylate group: In addition, the important characteristic must involve interaction with solvent as loss in intensity of vibrational bands is normally assigned to solvent-solute interactions.

The effect of solvent on vibrational bands is well known (126). If the solvent cage around the solute can form strong interactions with that solute, for example through hydrogen bonding, then the solvent will act to damp the molecular vibrations of the solute. Vibrational band broadening and weakening of absorbance is observed as the result of solvent-solute interactions; the stronger the interaction, the more pronounced is the observed effect. 
The solvents DMSO, THF and $\mathrm{CHCl}_{3}$ are all electron pair donors (Lewis bases): their relative strength is in the order of (DMSO> $\mathrm{THF} \mathrm{CHCl}_{3}$ ). The relative damping of the vibrational bands in $\mathrm{H}_{2}$ TAPP $^{0}$ is also in the order of $\mathrm{DMSO}_{\mathrm{THF}}>\mathrm{CHCl}_{3}$. Both the amino and carboxylate functional

\section{B TAPP $^{0}$ IN THF}

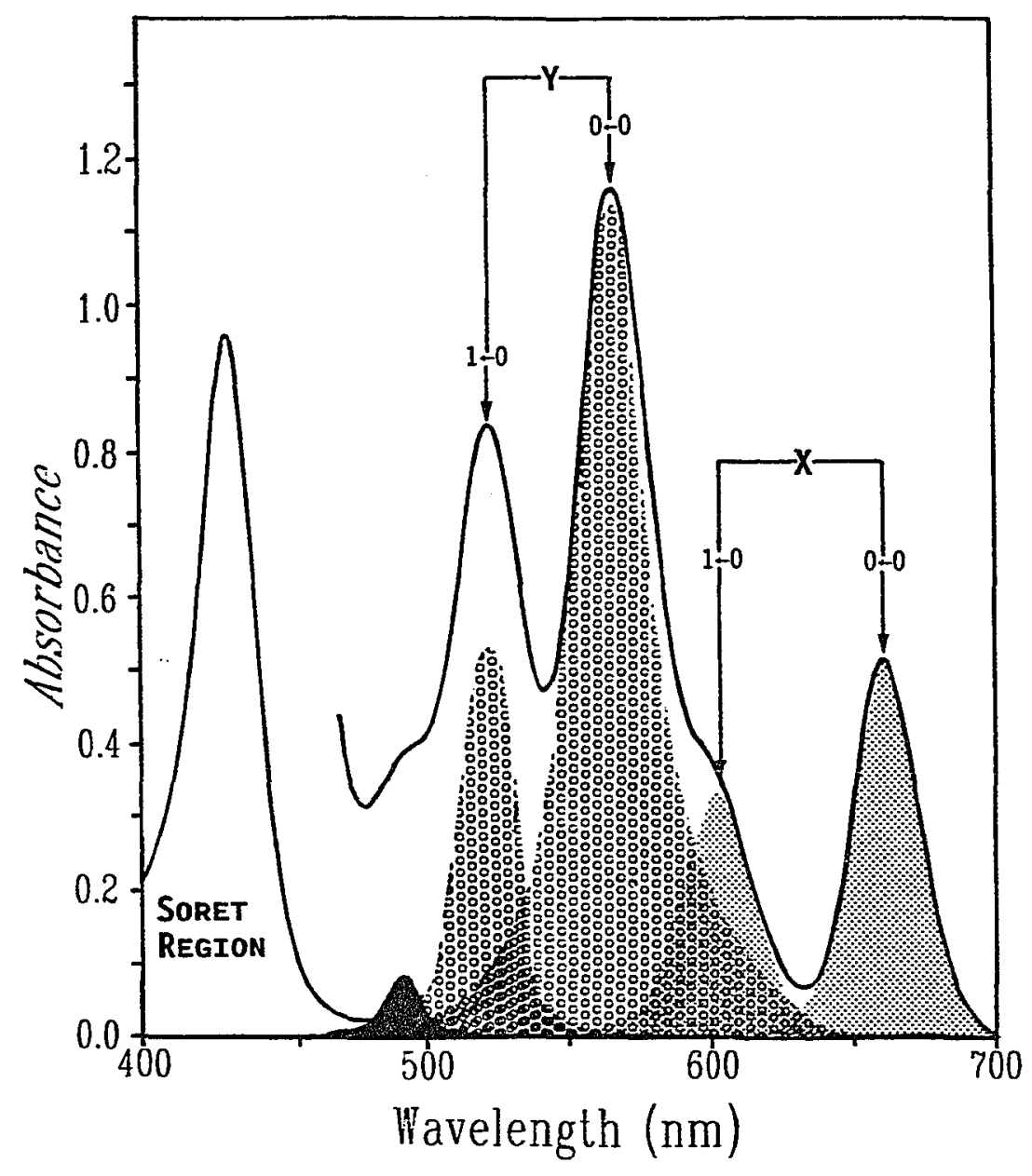

Figure 7. Spectrum of $B_{2}$ TAPP $^{0}$ in tetrahydrofuran. The $Z$ and $Y$ splitting of the $Q$ band region is shown. The $X$ components are shaded (保), the $Y$ components indicated by a pattern of (0); the peak areas and shapes are approximate. The black peak is unassigned. The $\left[\mathrm{B}_{2} \mathrm{TAPP}^{0}\right.$ ] in the soret region is $\approx 10 \mathrm{x}$ less than the concentration in the $Q$ band region. 


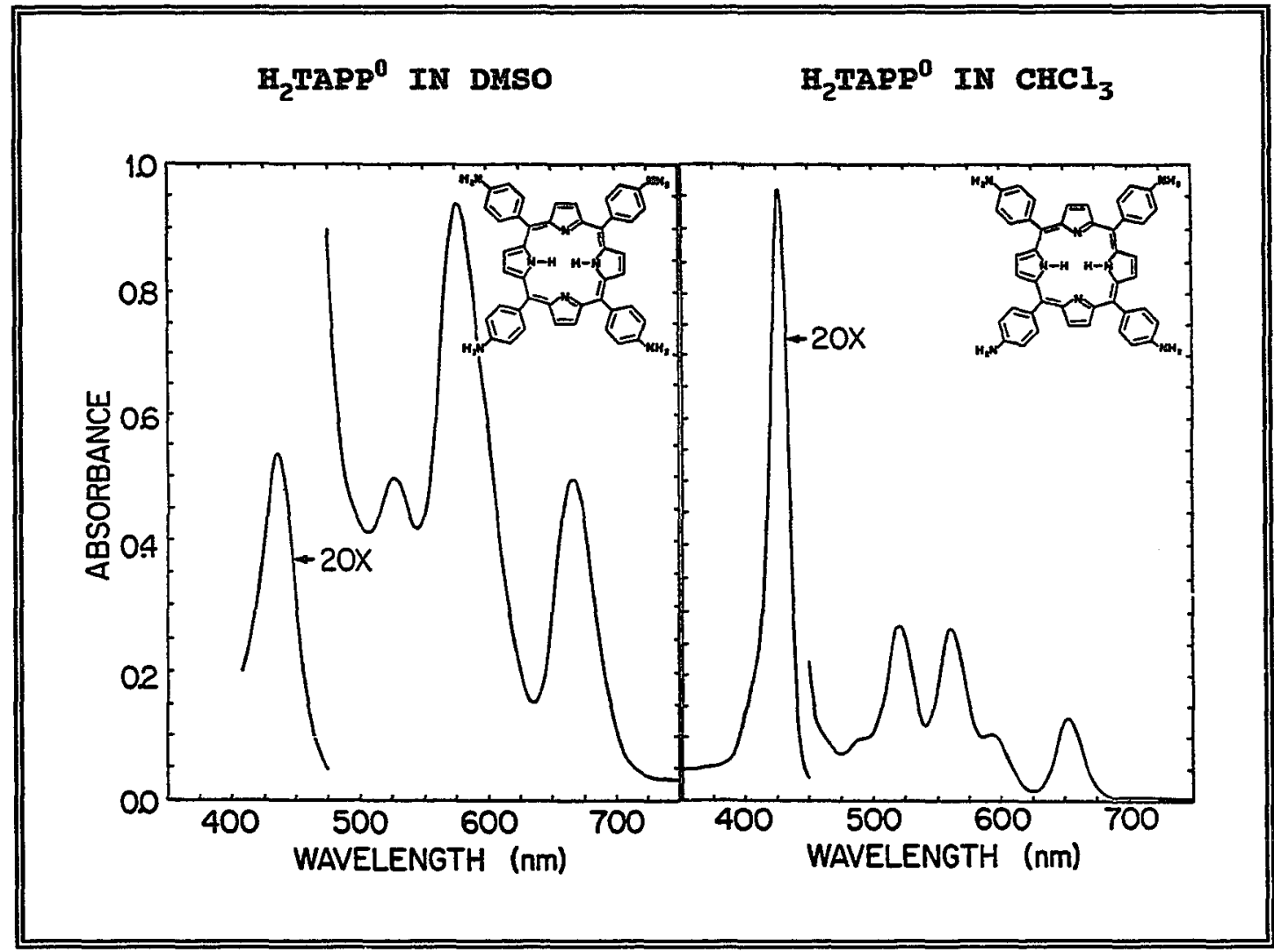

Figure 8. Spectra of H $_{2}$ TAPP $^{0}$ in dimethylsulfoxide and chloroform. The patterns in the $Q$ band region are shown. Notice that the patterns are solvent dependent.

carboxylate functional groups have polar bonds to hydrogen, thus should form hydrogen bonds with solvents which are electron pair donors (i.e. Lewis bases). These two porphyrins may differ in 1) the number of hydrogen bonds possible and 2) the stabilization of the hydrogen bonds. The electron-donating characteristics of the amino group result in a shift of electron density away from the nitrogen, leaving the group with a partial positive charge as compared to the carboxylate group which is electron withdrawing and more electronegative. Therefore, the amino group should stabilize hydrogen bonds more effectively than the carboxylate group. Figure 9 shows how resonance of the p-aminophenyl group, as compared with the p-carboxyphenyl group, could stabilize hydrogen bonding with the solvent DMSO. 
The Effect of $\mathrm{pH}\left(\mathrm{H}_{2} \mathrm{TAPP}^{0}\right)$. We conclude this section on porphyrin absorbance spectroscopy by comparing two spectral patterns; one which we can, and one which we connot explain. In Figure 10 a typical "dication" spectrum is shown $\left(\mathrm{H}_{4} \mathrm{TAPP}^{6+}\right.$ in $1 \mathrm{M} \mathrm{HCl}$, identical to Figure 2). The spectrum obtained at lower acid concentration shows a novel spectral pattern. Moreover, a similar effect is observed in organic

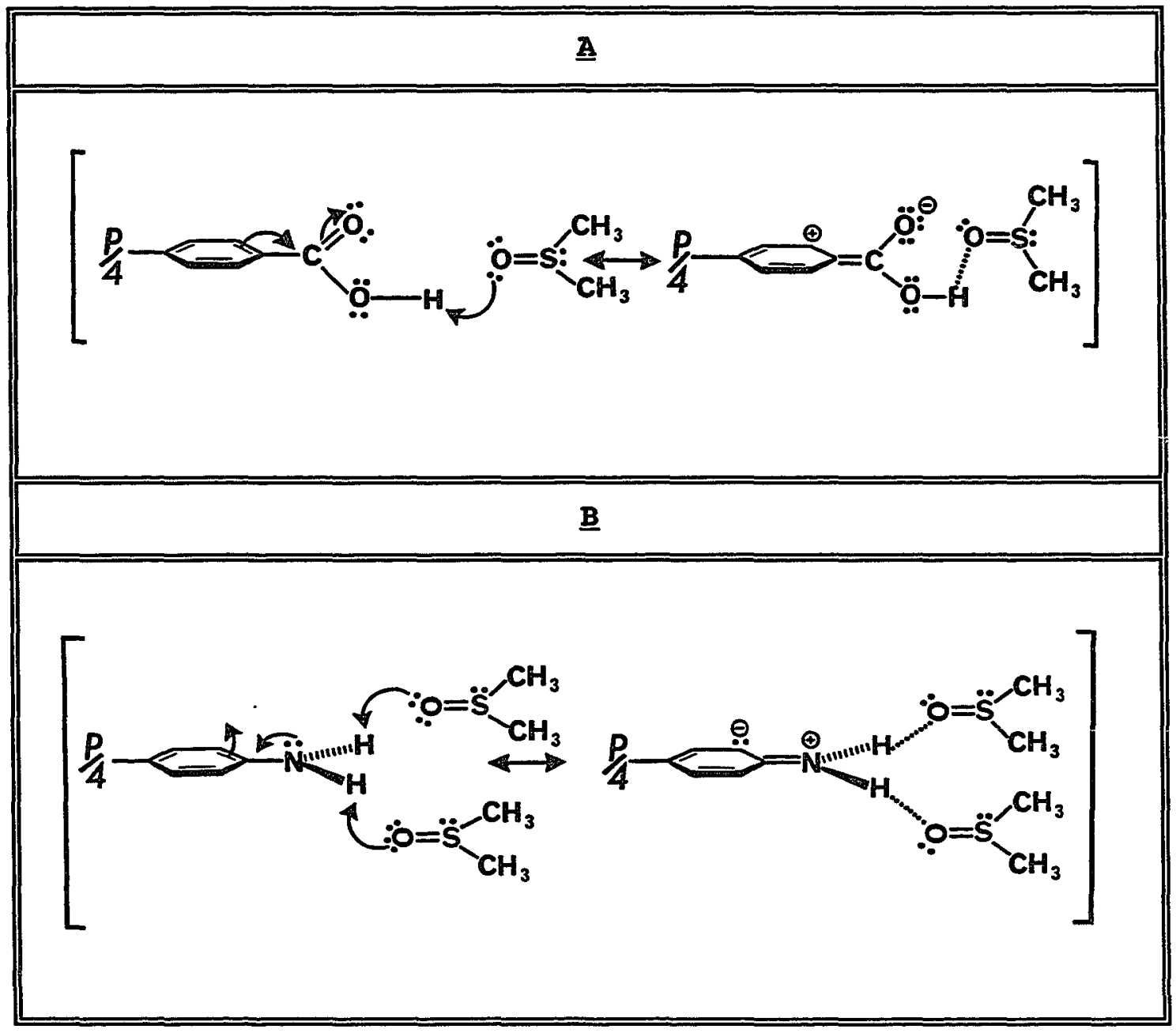

Figure 9. Possible rationalization for the solvent effects observed in $\mathrm{H}_{2} \mathrm{TAPP}^{0}$ but not in $\mathrm{H}_{2} \mathrm{TCPP}^{4-}$. P/4 indicates the porphine core and the three additional functional groups which are not shown. A) diagrams the effect for $\mathbf{H}_{2}$ TCPP $^{(}$, B) for $H_{2}$ TAPP 0 .

solvents as shown in Figure 11, where two novel peaks appear upon acid 
addition, these are shaded for emphasis.

If $1 \mathrm{M} \mathrm{HCl}$ produces the acidic "dication" $\left(\mathrm{H}_{4} \mathrm{TAPP}^{6+}\right)$, then $0.1 \mathrm{M}$ HCl would be expected to produce the "monocation" ( $\mathrm{H}_{3} \mathrm{TAPP}^{5+}$ ). The spectral pattern of the "monocation" has been variously reported by different authors. For hematoporphyrin, the soret band is blue-shifted and weakened (28). Three peaks are observed in the $Q$ band region; the lowest energy band usually observed at approximately $650 \mathrm{~nm}$ is blueshifted by approximately $40 \mathrm{~nm}$. Obviously, this does not fit the

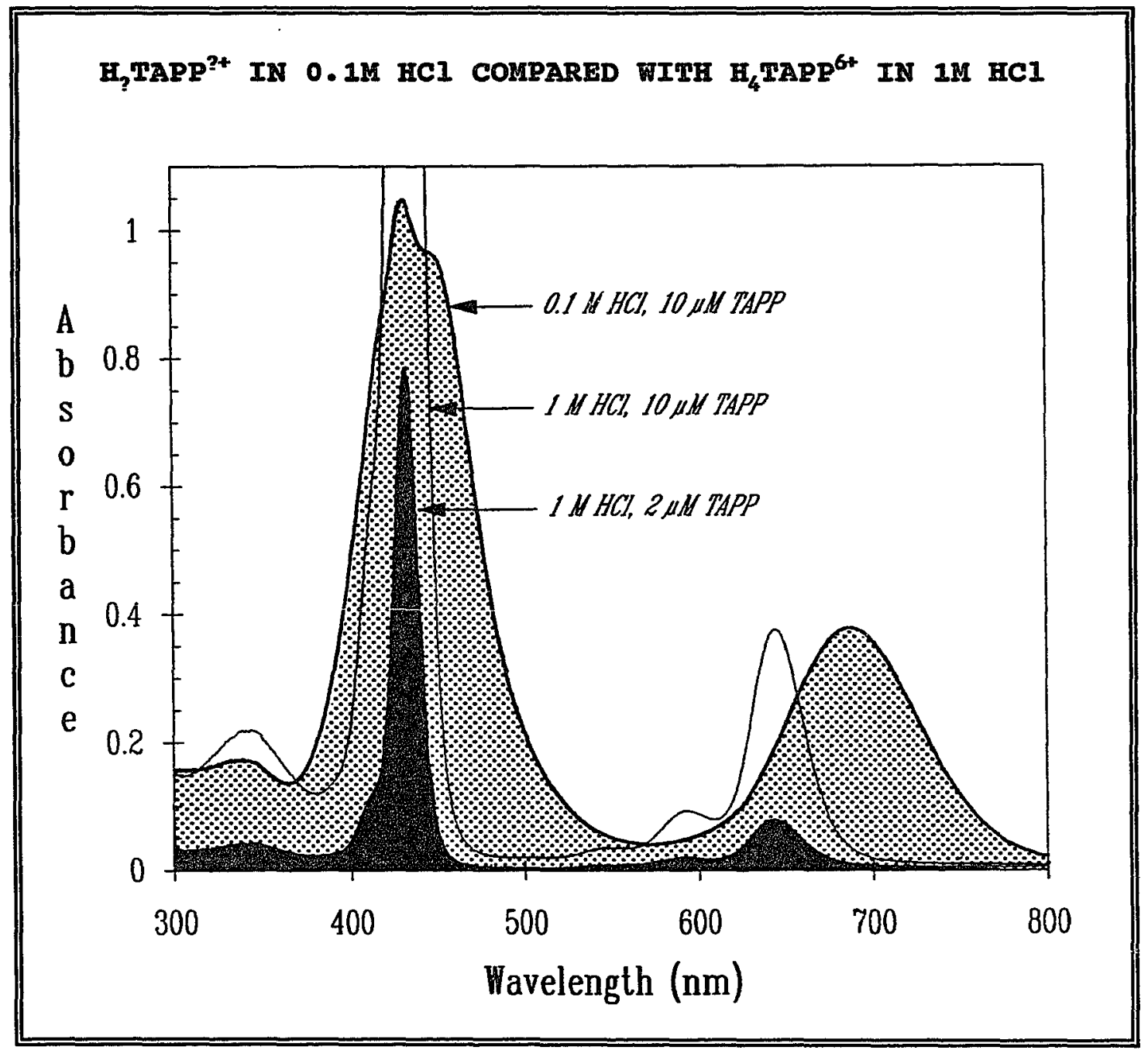

Figure 10. Spectra of $\mathrm{B}_{4}$ TAPP $^{6+}$ and $\mathrm{B}_{3}$ TAPP $^{\text {?t }}$ in acidic aqueous solution... The unexplained spectrum observed at 0.1 MCl is shaded (保), the normal acid spectrum $\left(D_{4 h}\right)$ in $1 M$ HCL is darkly shaded (㮁). 


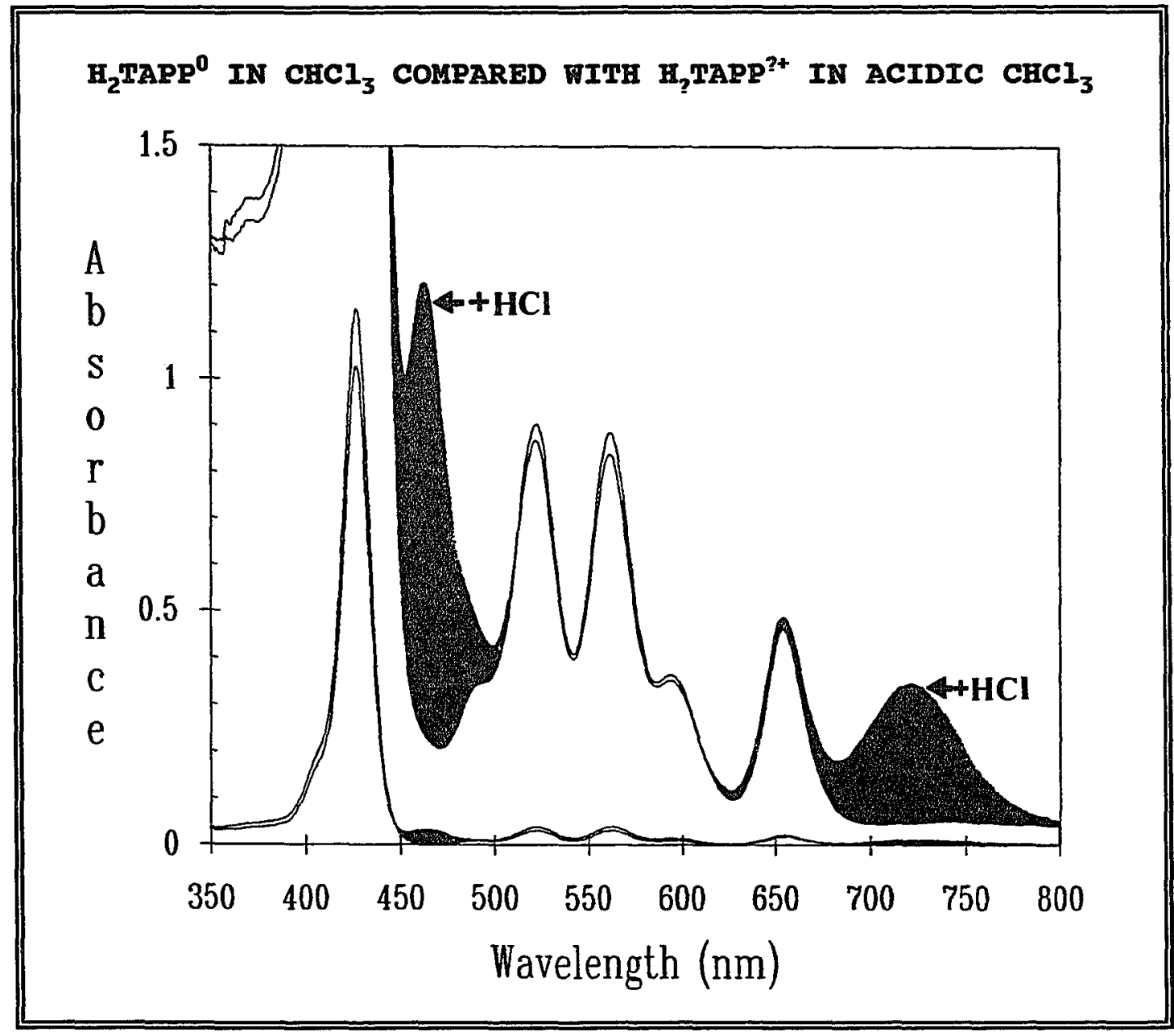

\footnotetext{
Figure 11. Spectra of $\mathrm{H}_{2}$ TAPP $^{0}$ in chloroform and $\mathrm{H}_{2}$ TAPP $^{2+}$ in acidic chloroform. For the set of spectra showing the $2-$ band region, the [porphyrin]=30 $\mu \mathrm{M}$; for the set showing the Soret region, [porphyrin] $=3 \mu \mathrm{M}$. The two novel peaks observed in acidic solution are shaded (․ㅜ‥
}

spectral pattern observed in $0.1 \mathrm{M} \mathrm{HCl} \mathrm{(Figure} \mathrm{10).}$

Rau and Longo (87) report the monocation spectrum for an analogous porphyrin, $\mathrm{H}_{2} \mathrm{TPyP}^{4+}$. These authors observe an absorbance loss for both the 517 and $638 \mathrm{~nm}$ bands upon formation of the monocation from the free base (they did not include a study of the soret band region). In addition, no band near $700 \mathrm{~nm}$ was observed. Thus, neither description of the monocation spectra fits the spectral patterns observed in Figures 10 and 11. 
It has been reported that the addition of a third proton into the central cavity to form the monocation is rapidly followed by the formation of the dication or by ring reduction (127). Reduced species called phlorins or chlorins (28) have very characteristic spectra (chlorophyll is an example of a chlorin). Typically, the soret band is red-shifted and weakened, and the $s_{0} \rightarrow s_{1}(0 \leftarrow 0)$ electronic transition is strengthened. Reduction should be favored by acidic conditions and facilitated if the p-aminophenyl group is conjugated with the porphine core, since resonance should increase the electron density in the ring. Even without reduction, a major perturbation would be expected if conjugation were to exist between the porphine core and the phenyl rings. This would affect the principal resonance path of the porphine ring by disrupting the Hückel aromatic system. An electron pair donated into the porphine core would result in an increase from 18 to $20 \pi$ electrons which is no longer an aromatic system by the Hückel criteria ( $20 \pi$ electrons is a $4 \mathrm{n}$ system, which is antiaromatic). The $20 \pi$ electron system is electronically similar to the chlorin. However, the spectrum in aqueous solutions at $0.1 \mathrm{M} \mathrm{HCl}$ and $1.0 \mathrm{M} \mathrm{HCl}$ appear to be distinct species rather than partial $(0.1 \mathrm{M})$ to complete $(1.0 \mathrm{M})$ conversion to a reduced species as would be expected.

The spectra in Figures 10 and 11 resemble the spectra of the $\pi$ anion $(36,38)$ and $\pi$ cation radicals $(78,128,129)$. Certain porphyrins have stable porphine ring cation and anion radicals; however, these are always measured under anoxic conditions. The solutions used to obtain the spectra in Figures 10 and 11 were in tightly closed (but not degassed) solutions making $\pi$ radical observation improbable. We do not know the solution species responsible for the unusual spectra shown in Figures 10 and 11; they do not appear to be the "monocation" species. The novel peaks immediately disappear upon the addition of base, thus if the chlorin or some electronically similar species is formed, it is stable only in acidic solutions. 
The Effect of $\mathrm{pH}\left(\mathrm{H}_{2} \mathrm{TCPP}^{4-}\right)$. We end this discussion of porphyrin absorbance spectroscopy by comparing the spectrum of free base $\mathrm{H}_{2} \mathrm{TSPP}^{4-}$ with the spectrum of its monocation $\left(\mathrm{H}_{3} \mathrm{TSPP}^{3-}\right)$. In Figure 12 the crosshatched spectrum shows $\mathrm{H}_{2} \mathrm{TSPP}^{4-}$ in equilibrium with the monocation in deionized water and minimal ionic strength at a pH of approximately 5 . The peak at $412 \mathrm{~nm}$ is the soret band of the free-base spectrum, the peak

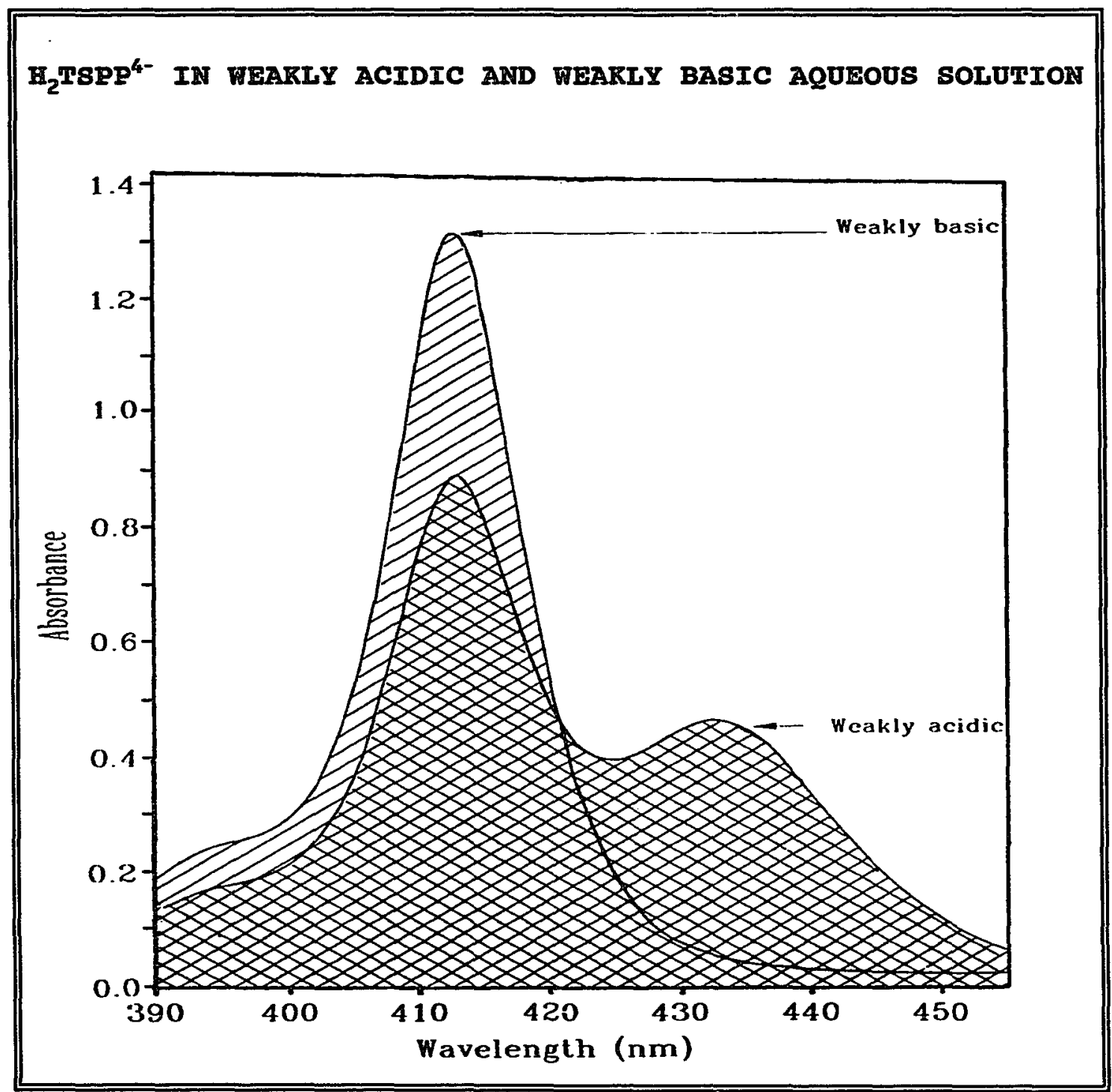

Figure 12. Spectra of H,TSPP ${ }^{4-}$ in weakly acidic and weakly basic aqueous solutions. The cross-hatched spectrum shows the equilibrium of the free base with the monocation; the striped spectrum is of the free base. 
at approximately $435 \mathrm{~nm}$ is the soret band of the monocation spectrum which is converted to the free-base upon the addition of base (Figure 12, striped spectra). The monocation spectrum has also been observed for $\mathrm{H}_{2} \mathrm{TCPP}^{4-}$ upon dilution of the basic stock solution; it is similarly abolished by the addition of base.

Iavalee (13) reported that the $\mathrm{pk}_{3}$ for the protonation of a third imino nitrogen (formation of the monocation) could be correlated to the rate of formation of water soluble porphyrin complexes of Cu(II). The estimated $\mathrm{pK}_{3}$ for the $\mathrm{H}_{2} \mathrm{TSPP}^{4-} / \mathrm{H}_{3} \mathrm{TSPP}^{3-}$ was approximately 5 , explaining the presence of both the free-base and the monocation species at $\mathrm{pH}$ values of approximately 5 as shown in Figure 12 .

Schmehl and coworkers (77) observe similar spectral changes between $\mathrm{pH}=3$ and $\mathrm{pH}=6.5$, but associate these changes with the protonation of the peripheral sulfonate groups. Their interpretation is consistent with the reported $\mathrm{pK}_{\mathrm{a}}$ (49) for the sulfonate groups, but I am not aware of any additional studies which correlate spectral changes with protonation of peripheral functional groups. Usually the acid/base chemistry of the functional groups is associated with porphyrin monomer/dimer equilibria.

\section{NUCLEAR MAGNETIC RESONANCE SPECTROSCOPY}

\section{Porphyrin ${ }^{1}$ H MMR Spectra}

As in the availability of literature concerning the absorbance spectroscopy of porphyrins, most of the nuclear magnetic resonance (NMR) studies have similarly focused on the metalloporphyrins $(130,131)$. However, NMR studies have been invaluable in clarifying the solubility of certain substituted tetraphenylporphyrins. For example, it was thought initially that $\mathrm{H}_{2} \mathrm{TMPyP}^{4+}$ existed in monomer form into the $\mathrm{mM}$ concentration range in aqueous solution $(62,83-86)$; recently this porphyrin has been shown to exist as a dimer into the $0.1 \mu \mathrm{M}$ concentration regions by Kano et al. via fluorescence (63) and $400 \mathrm{MHz}$ 
$1_{\mathrm{H}}$ NMR (58), which was confirmed by Harriman et al. (87) by absorbance spectroscopy. In addition, Corsini and Herrmann (89) have extended the investigation of the speciation of $\mathrm{H}_{2} \mathrm{TSPP}^{4-}$ into the $100 \mathrm{mM}$ concentration range by the application of NMR methodology. We have used the assignments for $\mathrm{H}_{2} \mathrm{TSPP}^{4-}$ (89) to make our assignments for $\mathrm{H}_{2} \mathrm{TCPP}^{4-}$ (Figure 13).

The resonance at a particular energy allows identification of protons in unique internal magnetic environments. The peaks in NMR are separated by very small energy increments, thus are reported on the energy scale as parts-per-million (ppm) termed "chemical shift" or " $\delta "$, relative to an arbitrary standard, tetramethylsilane (TMS), defined as 08. The range of the NMR spectra of free base porphyrins is generally from -3 to $+10 \mathrm{ppm}(130)$. The peak resolution is dependent upon the strength of the external magnetic field, $\mathrm{H}_{0}$, thus higher magnetic fields are often able to resolve peaks which are superimposed at lower magnetic field strengths. The integrated intensity is proportional to the number of protons in a particular internal magnetic environment. In this study we are more interested in $\delta$ than in the integrated intensity. The NMR spectra of porphyrins are very sensitive to solvent, concentration, and instrumental differences making comparisons of data from different laboratories difficult. Despite the apparent differences, several general trends in the ${ }^{1} \mathrm{H}$ NMR spectra of free base porphyrins are observed; thus assignments by analogy to the literature are often possible for substituents on the porphyrin macrocycle (130). Ring current effects contribute most to the character of the chemical shifts observed; thus, as for any annulene, the protons on the interior of the ring (on the imino nitrogens) are shifted upfield above TMS, and the protons on the exterior are shifted downfield. For the tetraphenylporphyrins, the phenyl protons are influenced by a combination of the porphine and phenyl ring currents. The phenyl ortho protons are in closest proximity to the porphine core, but resonate 
upfield relative to the phenyl meta protons as these are near the electronegative carboxylate group. In $\mathrm{H}_{2}$ TPP the relative resonances of the phenyl meta and ortho protons are reversed (130).

Figure 13 shows the $300 \mathrm{MHz}^{1} \mathrm{H}$ NMR spectrum of $0.50 \mathrm{mM} \mathrm{H} \mathrm{TCPP}^{4-}$ in $\mathrm{D}_{2} \mathrm{O}$ at $\mathrm{pD} \approx 7$ in $\mathrm{KD}_{2} \mathrm{PO}_{4} / \mathrm{NaKDPO}_{4}$. There are four sets of protons in different magnetic environments; the labels and descriptions which follow correspond to the illustration of the chemical structure of $\mathrm{H}_{2} \mathrm{TCPP}^{4-}$ in the figure below: B) $8 \mathrm{~B}$-pyrrole protons, $m$ ) 8 protons in the meta position on the carboxyphenyl groups, 0) 8 protons in the ortho position on the carboxyphenyl groups, and $\alpha) 2$ protons on the inner imino nitrogens (which do not appear in the NMR spectrum in $D_{2} O$ as these protons exchange with deuterium). The resonance at approximately 7 ppm is due to the $\beta$-pyrrole protons of the face-to-face porphyrin dimer (89).

In addition to information on the identification of organic molecules, $1_{H}$ NMR spectroscopy gives excellent information concerning association chemistry, either dimerization or complexation. The strong porphine ring currents can act at short distances to alter the chemical shifts of molecules involved in association complexes with porphyrins. In addition, information concerning the orientation of the molecule associated with the porphyrin can be acquired, since the shifts will be upfield if the molecule is over the interior of the porphine core and downfield if the molecule is over the exterior. From ${ }^{1} \mathrm{H}$ NMR it was learned that porphyrins can form slightly off-set face-to-face dimers (68).

In Chapter IV evidence is shown via $300 \mathrm{MHz}$ NMR that $\mathrm{MV}^{2+}$ dissociates $\mathrm{H}_{2} \mathrm{TCPP}^{4-}$ dimers by correlating the sharpening of the resonance of the phenyl ortho protons to face-to-face porphyrin dimer dissociation $(68,89)$. The peak from the aggregated form is shifted downfield and broadened relative to the monomer NMR spectra. The sensitivity of this technique is too low to detect $\mathrm{H}_{2} \mathrm{TCPP}^{4-}$ within the 


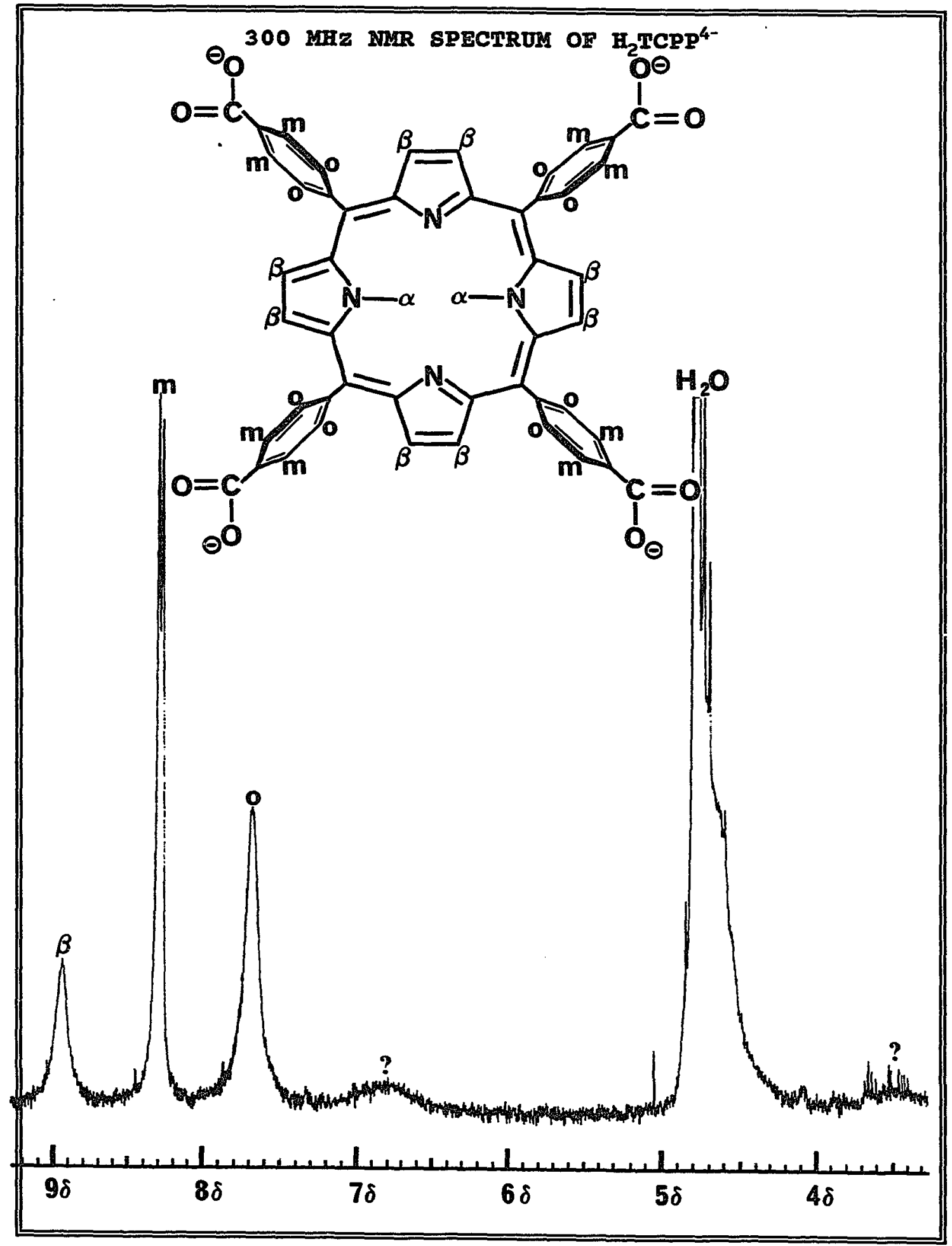

Fiqure 13. $300 \mathrm{MHz}{ }^{1} \mathrm{H}$ NMR spectrum of $\mathrm{H}_{2} \mathrm{TCPP}^{4-}$ in $\mathrm{D}_{2} \mathrm{O}$ at $\mathrm{pD}=7$, buffered with $5 \mathrm{mM} \mathrm{KD}_{2} \mathrm{PO}_{4} / \mathrm{NaKDPO}_{4} \cdot$ 
concentration range where it is in monomer form only in aqueous solution at neutral pH values. Even with the use of new technologies, we predict that the sensitivity will probably be extended only into the $10 \mu \mathrm{M}$ concentration range, an order of magnitude higher than that most useful to the study of $\mathrm{H}_{2} \mathrm{TCPP}^{4-} /$ viologen complexation.

\section{VIOLOGEN ${ }^{1}$ H NMR SPECTRA}

The ${ }^{1}$ H NMR spectra of the four viologens listed in Table II are shown in Figures 14 and 15. For the symmetrically $\mathrm{N}$-substituted bipyridyls there are two sets of four equivalent pyridyl protons yielding an NMR spectrum of two doublets shifted well downfield relative to the resonance(s) of the $\mathrm{N}$-substituent (132). The asymmetrically Nsubstituted bipyridyls have four sets of two equivalent pyridyl protons yielding four doublets downfield from the N-substituent peaks.

Although the sensitivity of ${ }^{1} \mathrm{H}$ NMR is too low to detect $\mathrm{H}_{2}$ TCPP $^{4-}$ in the concentration region used for the porphyrin/viologen titrations described in chapter $v$, the minimal relevant viologen concentration is an order of magnitude higher than the porphyrin. Therefore, it is possible that equilibrium constants could be determined via NMR if the changes in the chemical shifts of the viologen were followed during the progress of the titration. Since a $400 \mathrm{MHz}$ NMR spectrophotometer is now available at PSU, we may explore this technique in later studies. 


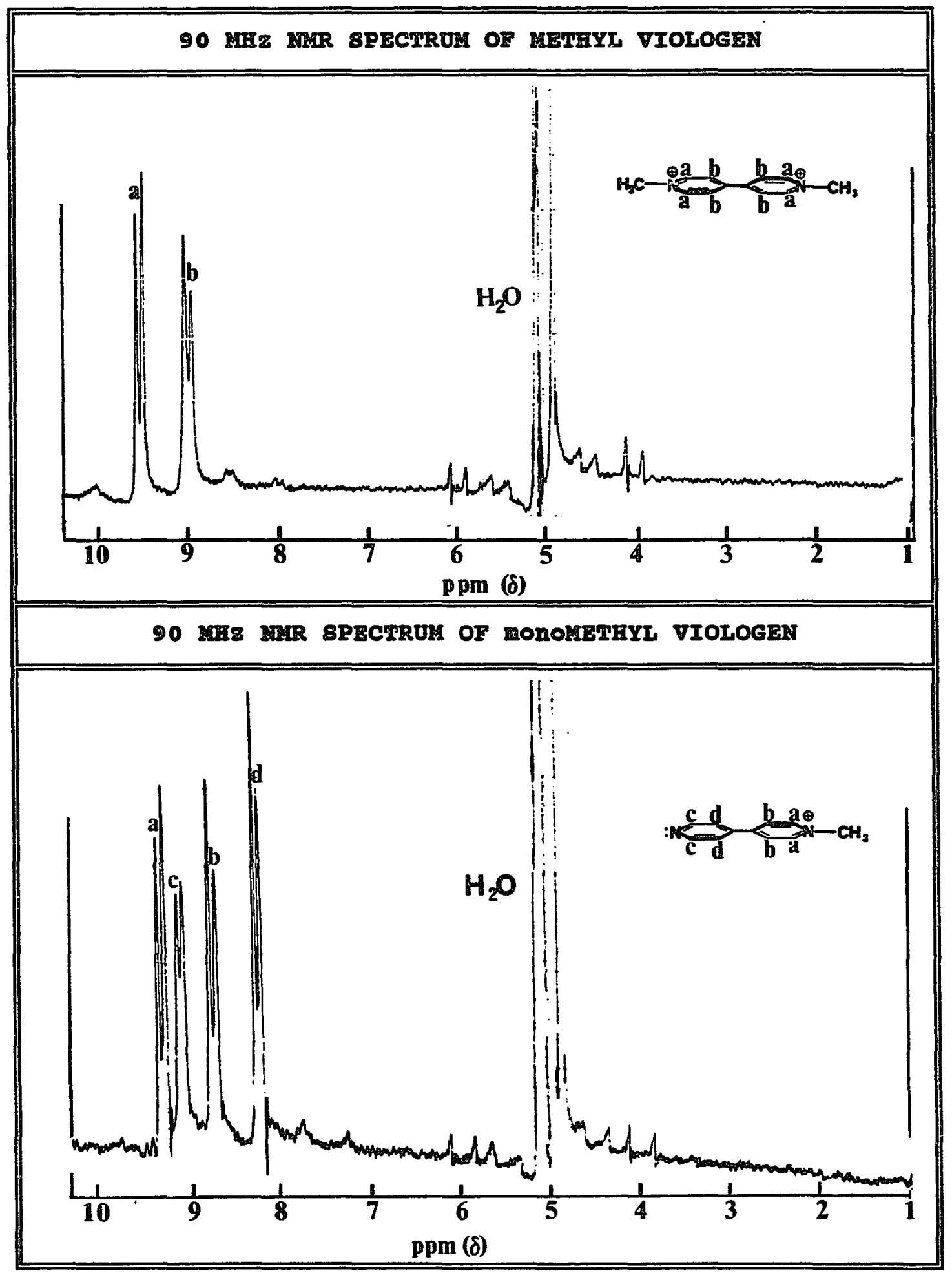

Figure 14. $90 \mathrm{mHz}^{1}{ }^{1 \mathrm{H}} \mathrm{MMR}$ spectra of $\mathrm{Mv}^{2+}$ and $\mathrm{mMV}^{1+}$ in $\mathrm{D}_{2} \mathrm{O}$. 


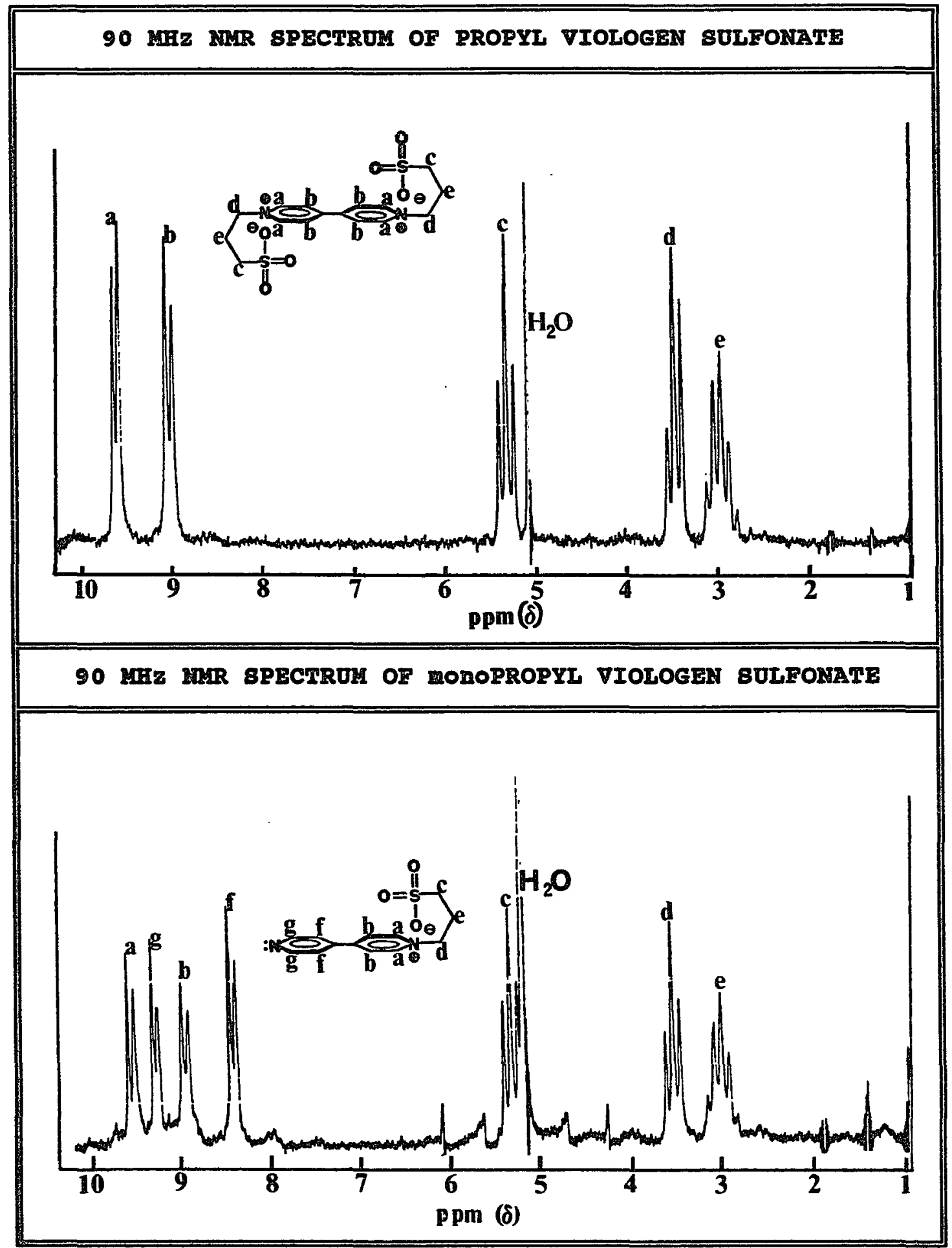

Fiqure 15. $90 \mathrm{MHz}{ }^{1} \mathrm{~B}$ MMR spectra of $\mathrm{PVS}^{0}$ and mPVs ${ }^{0}$ in $\mathrm{D}_{2} \mathrm{O}$. 
ChAPTER III

MATERIAIS AND METHODS

\section{INTRODUCTION}

This section contains all syntheses, purification schemes, instrumental methods, and mathematical methods for data analysis.

\section{SYNTHESIS AND/OR PURIFICATION OF MATERIAIS}

\section{Purification of Commercial Porphyrins}

Several authors have noted that porphyrins co-crystallize $(133,153)$ with many organic solvents, yet few examples exist where these trapped solvents are removed, even though it is a simple procedure. It has been shown that small concentrations of certain organic solvents, such as pyridine, cause the faces of linked dimers to move apart (61) and induces dimer dissociation (68). In order to avoid concern that the porphyrin/viologen equilibria might be affected by small amounts of organic solvents, the solvents were removed as described below.

Purification of $\mathrm{H}_{2} \mathrm{TCPP}^{4-}$. The purity of $\mathrm{H}_{2} \mathrm{TCPP}^{4-}$ (acid form) purchased from both Strem Chemicals and Porphyrin Products was compared by high pressure liquid chromatography (HPLC) for the presence of multiple porphyrin species (experiment performed by Robert Ransdell). $\mathrm{H}_{2} \mathrm{TCPP}^{4-}$ purchased from Porphyrin Products eluted as a single band, thus was considered not to be contaminated by other porphyrin species.

The sample was checked for organic impurities via $90 \mathrm{MHz}{ }^{1} \mathrm{H}$ NMR (Varian model EM390) in $d_{5}$-pyridine and found to be contaminated with dimethylformamide (DMF) and acetic acid. Residual solvents were removed by volatilization under high vacuum (approximately $10^{-5}$ Torr) by the following procedure: 
The one gram sample was ground in an agate mortar before division into approximately $250 \mathrm{mg}$ aliquots. Each aliquot was placed into a small glass container and covered with filter paper (secured by copper wire). The vials and a small thermometer were placed into a roundbottom flask fitted with a heating mantle and half filled with ottawa sand (to increase thermal conduction). The flask was protected from light and connected to the high vacuum line; after 24 hours at $20^{\circ} \mathrm{C}$ the high vacuum was reestablished indicating that the most volatile solvents had been removed. The temperature was gradually increased until the vacuum was observed to drop; when high vacuum was reestablished the cycle was repeated until a large temperature increment (ca. $25^{\circ} \mathrm{C}$ ) did not result in a loss of vacuum. The maximum temperature in the sand bath was $100^{\circ} \mathrm{C}$; the procedure took four days. The (near) absence of contaminants was confirmed by $1_{H}$ NMR in $d_{5}$-pyridine and $d_{6}$-dimethylsulfoxide.

The sample was found not to contain detectable quantities of trace metals by atomic emission spectroscopy using silicon as a standard. The concentration of metals other than the standard was estimated by comparison of the silicon intensity with the intensity of the other species. The intensities were manipulated using a table of relative intensities of various metals at specific wavelengths (134) to yield the approximate concentration of a series of trace metals in the porphyrin sample.

Purification of $\mathrm{H}_{2}$ TSPP $^{4-} \cdot \mathrm{H}_{2} \mathrm{TSPP}^{4-}$ was purchased from Porphyrin Products as the sodium salt. The purity was checked by HPLC and the solvent impurities were removed as described for $\mathrm{H}_{2} \mathrm{TCPP}^{4-}$ except that the porphyrin was not heated above $40^{\circ} \mathrm{C}$ due to concern that the sulfonate groups might be lost as $\mathrm{so}_{3}$.

Synthesis of $\mathrm{H}_{2}$ TAPP4+

This porphyrin is currently commercially available although very expensive (ca. $\$ 150.00 /$ gram); however, during the first year of the 
artificial photosynthesis project in our lab (71), we had to rely on our own synthesis product. To the best of our knowledge there is still no published synthesis protocol which includes all the steps necessary for successful synthesis of $\mathrm{H}_{2} \mathrm{TAPP}^{0}$. For this reason we have described our synthesis protocol in some detail.

After several unsuccessful attempts at following the synthesis method reported by Thomas and Martel $(17,18)$, who emphasize the difficulty and low yields associated with this synthesis, and after incorporating suggestions from other authors (19-26), we were generously given an excellent and detailed synthesis protocol by Peter Hambright (135). The synthesis was performed in two steps: 1) synthesis of tetrakis $(p-n i t r o p h e n y l)$ porphine $\left(\mathrm{H}_{2} \mathrm{TNPP}^{0}\right)$ from the condensation of pyrrole and $p$-nitrobenzaldehyde, 2) reduction of $\mathrm{H}_{2} \mathrm{TNPP}^{0}$ to $\mathrm{H}_{2} \mathrm{TAPP}^{4+}$ using $\mathrm{SnCl}_{2}$.

step 1 was complicated by both the reactivity of pyrrole, which yields pyrrole polymers of various lengths (black tar), and the multiple products formed by side reactions. The overall reaction and several possible side-reactions are shown in Figure 16 below. We also show a plausible mechanism (Figure 17) to illustrate that within each major step in the reaction mechanism, side-reactions are possible which lower the yield.

Step 2 was complicated by the need to separate the reducing agent from the porphyrin product; this step was difficult as both have similar solubilities.

In our hands, the final yield of $\mathrm{H}_{2} \mathrm{TAPP}^{4+}$, after the associated solvents had been removed, was never greater than $10 \%$ and averaged closer to $7 \%$.

Step 1. Synthesis of HTHPP ${ }^{0}$. Unfortunately, this step could not, to our knowledge, be done on a larger scale as the yields drop. We think the reason for the drop in yield is that although more porphyrin is produced, the ratio of product to tar drops, thus less is recovered 
due to loss during the DMF washing step.

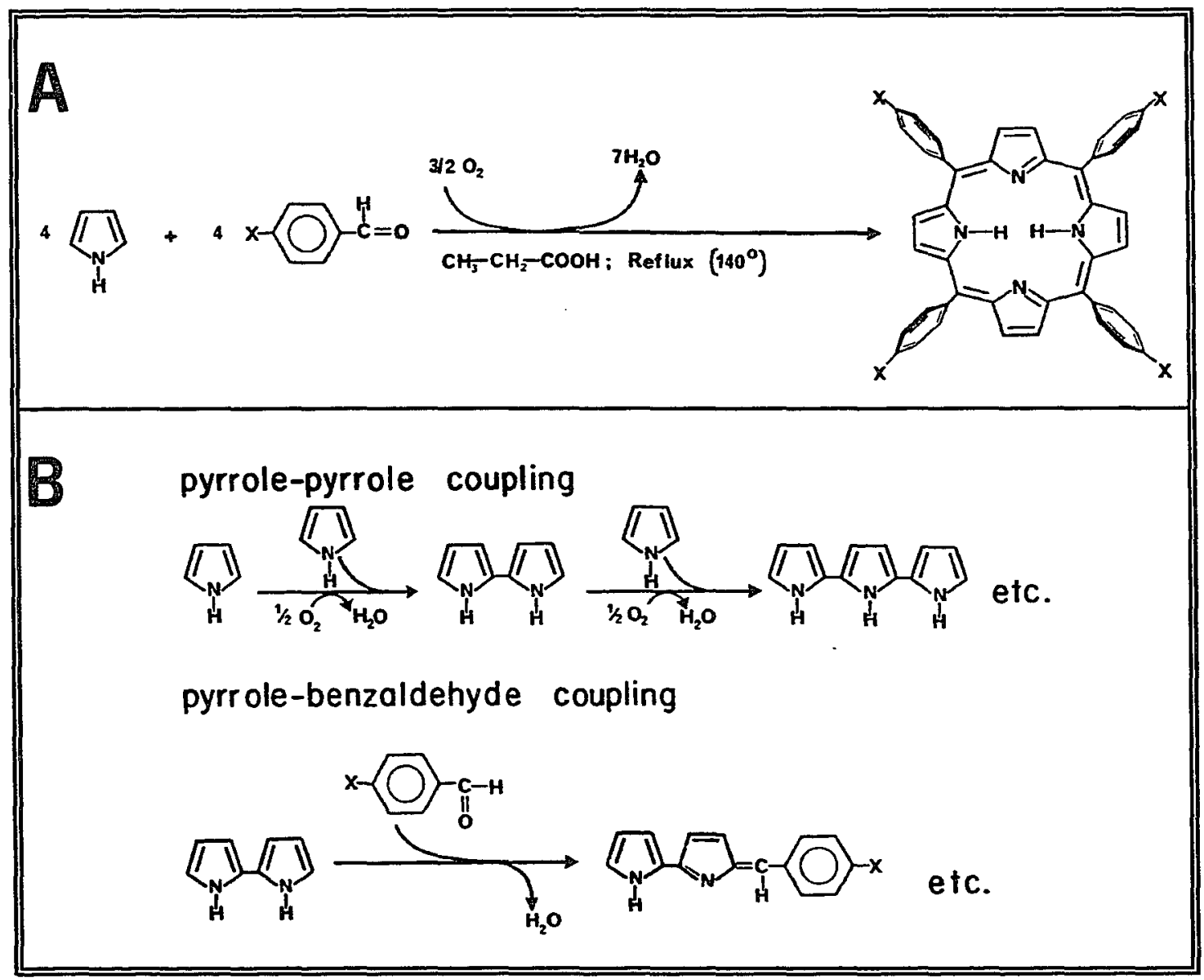

Figure 16. Overall reaction in the synthesis of a substituted tetraphenylporphyrin and two of the possible side-reactions. "A" shows the overall reaction between pyrrole and $p-$ nitrobenzaldehyde, where $\mathrm{Z}=\mathrm{NO}_{2}$. "B" shows two specific sidereactions.

Synthesis Protocol. The following was brought to reflux in a $500 \mathrm{ml}$ round-bottom flask: $5.5 \mathrm{~g}$ of p-nitrobenzaldehyde, $6 \mathrm{ml}$ of acetic anhydride, and $150 \mathrm{ml}$ of propionic acid. The mixture was stirred using a mechanical mixer. Freshly distilled (colorless) pyrrole $(2.5 \mathrm{ml})$ was added under the surface of the liquid in the flask to reduce exposure of the pyrrole to acid vapor before it has a chance to contact $p$-nitrobenzaldehyde. The mixture was refluxed for 30 minutes and left to cool to 


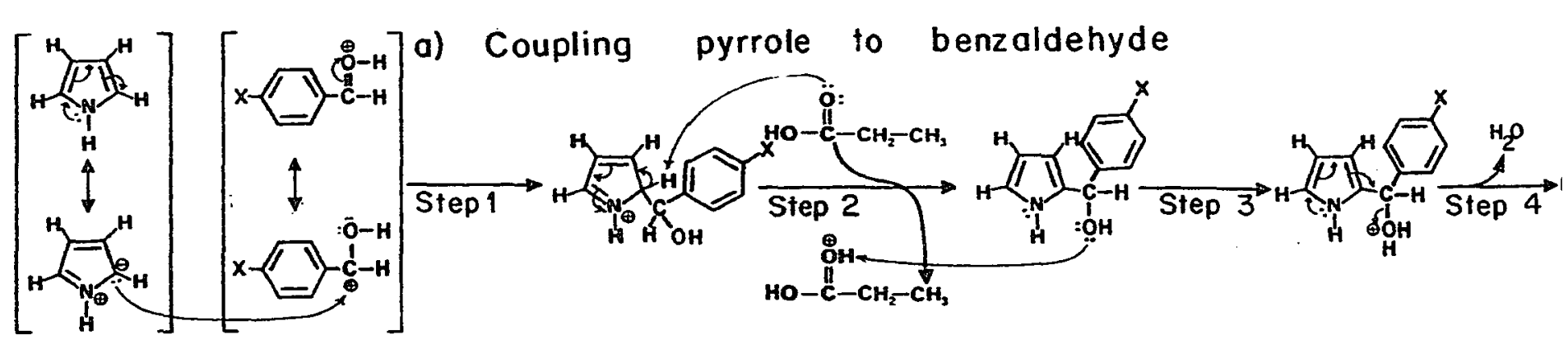

c) Coupling of athird pyrrole and benzaldehyde

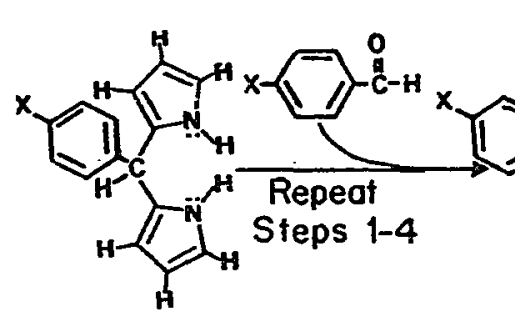

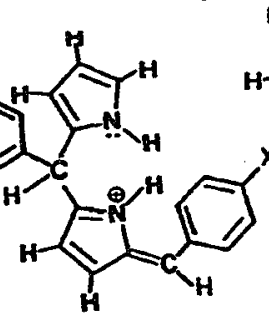

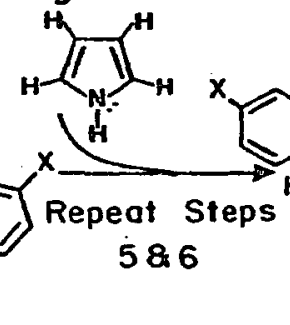

e) Ring Closure

e)

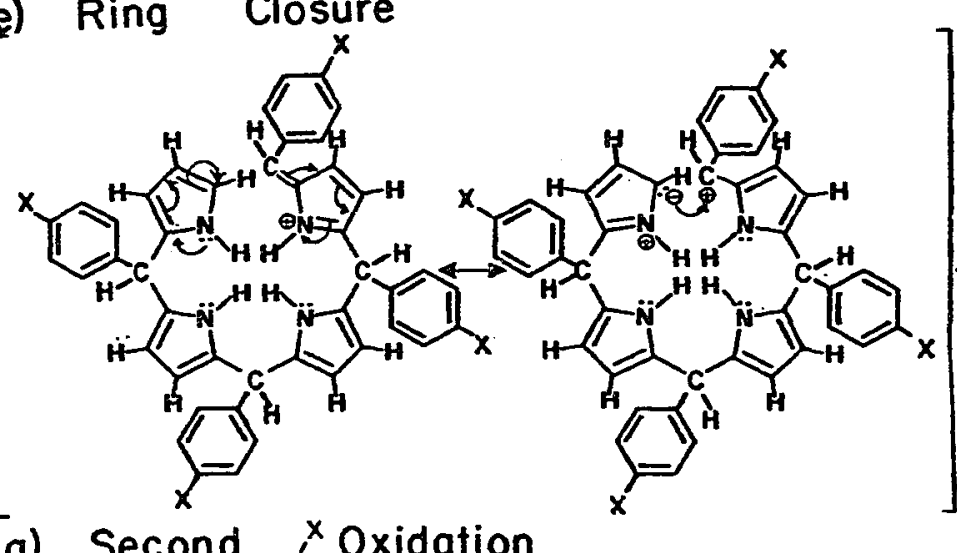

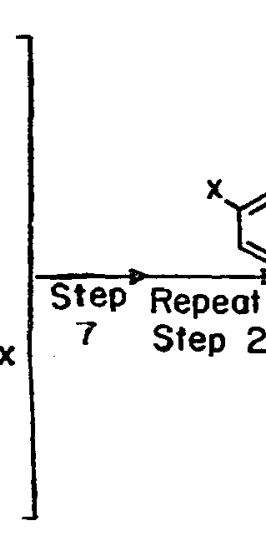

g) Second $x^{x}$ Oxidation

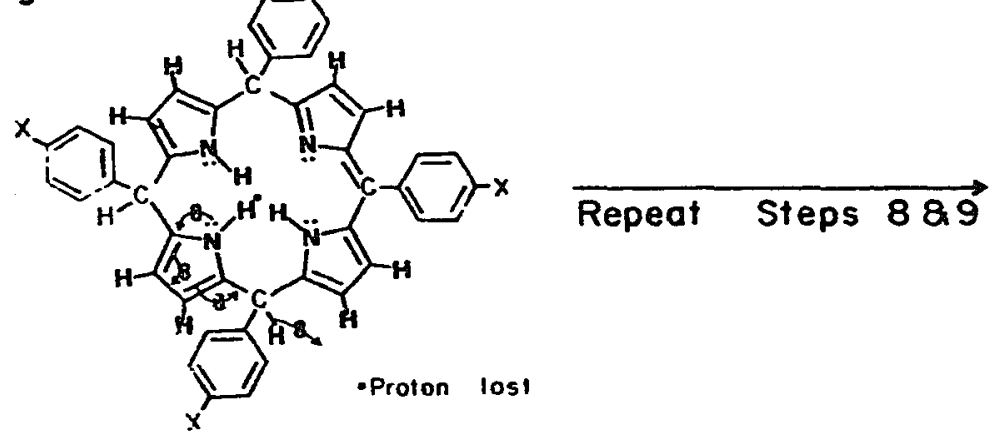

h) Third $x$ Oxidation

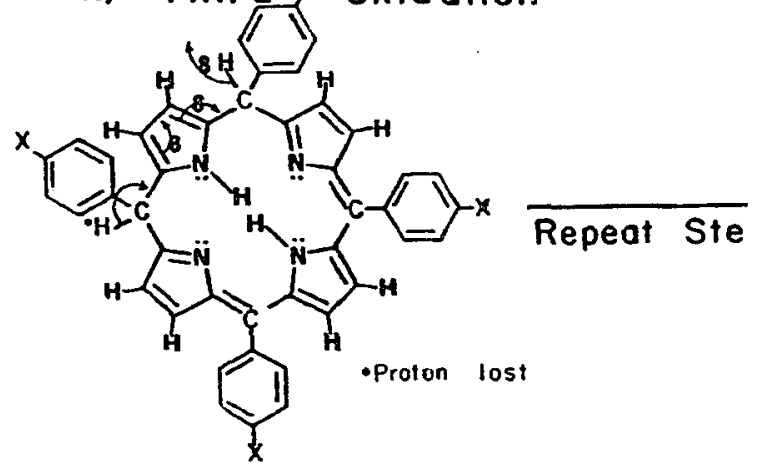

Figure 17. A plausible mechanism for the synthesis of Hit TAPP ${ }^{4+}$ The initial condensation reaction can be described as a nucleophilic substitution of pyrrole onto the carbonyl carbon of $p$-nitrobenzaldehyde. 


$=$



room temperature overnight. The cooled reaction mixture was a black, sticky, semiliquid with barely discernable dark purple crystals.

Purification of HIMPP ${ }^{0}$. The by-products and tars were removed at this step as $\mathrm{H}_{2} \mathrm{TNPP}^{0}$ was more readily purified than $\mathrm{H}_{2} \mathrm{TAPP}^{4+}$. This protocol took advantage of the extreme insolubility of the product in aqueous solution versus the solubility of many of the polar by-products. In addition, $\mathrm{H}_{2}$ TNPP $^{0}$ was sparingly soluble in DMF in contrast to the tars which were quite soluble in this solvent. Peter Hambright (135) recommended harvesting the tar and product via vacuum filtration in a sintered glass funnel, but we found this to be an extremely slow process. We harvested the insoluble material via centrifugation. The mixture was then rapidly washed in hot water and recovered by centrifugation as many times as was needed to remove the water-soluble colored by-products.

After drying on a watch glass, the sample was mixed with $20 \mathrm{ml}$ of DMF and the insoluble fraction recovered via centrifugation. This was repeated as necessary until the product was a bright dark purple. The amorphous purple precipitate was transferred to a sintered glass funnel for a final water wash.

The air-dried product was recrystallized from pyridine and washed with hot water followed by hexane. An accurate calculation of the yield required that the entrapped solvents be removed by volatilization, as described for previously for $\mathrm{H}_{2} \mathrm{TCPP}^{4-}$. From a single synthesis the yield of $\mathrm{H}_{2}$ TNPP $^{0}$ was calculated to be $12.7 \%$ after removal of entrapped solvents. This was compared with 6 subsequent syntheses not dried under vacuum where the combined yield of $17 \%$ was higher.

Characterization of $\mathrm{H}_{2}$ INPP ${ }^{0}$. The product was identified as $\mathrm{H}_{2}$ TNPP $^{0}$ due to an $\mathrm{R}_{\mathrm{f}}=0.54$ via thin layer chromatography (TLC) on silica plates developed in 1:1 pyridine:decane which agreed with the published value (17). In addition, the product yielded UV/VIS spectra in chloroform which agreed with published values: experimental 
absorption maxima $=646,590,551,515$, and $423 \mathrm{~nm}$ as compared with published values $(17)=646,590,551,516$, and $424 \mathrm{~nm}$, respectively.

Step 2. Synthesis of $\mathrm{F}_{2}$ TAPP $^{4+}$ from H$_{2}$ THPP ${ }^{0}$. The product from six syntheses of $\mathrm{H}_{2}$ TNPP $^{0}$ (two syntheses performed by Brian Johnson) yielding $6.94 \mathrm{~g}$ (which was an over estimate since the solvents were not removed) was combined for the scaled-up reduction described below.

Reduction of HTWPP $_{2}$. The reduction was performed as follows: in a stirred round-bottom flask fitted with a reflux condenser, $315 \mathrm{ml}$ of concentrated $\mathrm{HCl}, 27.3 \mathrm{~g}$ of stannous chloride (dihydrate), and $6.6 \mathrm{~g}$ $\mathrm{H}_{2} \mathrm{TNPP}^{0}$ were combined. The mixture was quickly heated to $80^{\circ} \mathrm{C}$ in a hot water bath and the reaction was allowed to proceed for 30 minutes. After cooling to room temperature, $315 \mathrm{ml}$ of deionized water was added. When too little water was added the porphyrin did not precipitate; when too much water was added the stannous (and stannic) chloride precipitated and much of the porphyrin redissolved.

This step relied on slight changes in pH combined with salting-out to precipitate $\mathrm{H}_{2} \mathrm{TAPP}^{4+}$ and has not been optimized.

The product was recovered via suction filtration on a sintered glass funnel and washed with deionized water. The sample was redissolved in water and neutralized with aqueous ammonium hydroxide until the solution changed from green (acidic) to brown (flocculent precipitate, this porphyrin was insoluble above $\mathrm{pH}=2$ ). We did not try using NaOH to neutralize the acid, this base was recommended over ammonium hydroxide by Dr. Ann Walker (personal communication). The porphyrin was recovered by suction filtration and washed two times with water before being left to dry in a vacuum desiccator. At this point the product was still contaminated with tin and partially reduced $\mathrm{H}_{2} \mathrm{TNPP}^{0}$. The amount of the contamination appeared to vary from experiment to experiment.

Purification of $\mathrm{H}_{2}$ TAPP $^{0}$ Via Recrystallization. Due to the low solubility of $\mathrm{H}_{2} \mathrm{TAPP}^{0}$, the recrystallization was performed in small 
batches. The porphyrin was dissolved in a minimal amount of hot chloroform and allowed to cool under vacuum. The crystals were then harvested via suction filtration. Alternatively, pure $\mathrm{H}_{2} \mathrm{TAPP}^{0}$ was obtained by dissolving in hot pyridine, cooling, and forcing out of solution by the addition of a few drops of hexane. These recrystallization (or precipitation) methods may not remove the partially reduced $\mathrm{H}_{2} \mathrm{TNPP}^{0}$. The entrapped solvents were removed $\mathrm{via}$ volatilization as described for $\mathrm{H}_{2} \mathrm{TCPP}^{4-}$.

Purification ox H TAPP $^{0}$ Via Column Chromatography. Column chromatography (not optimized) on silica gel developed in chloroform removed the metal and partially reduced $\mathrm{H}_{2} \mathrm{TNPP}^{4+}$, but resulted in large losses of porphyrin as the cationic porphyrin bound to anionic sites on the gel. Evidence from IR (spectra taken and analyzed by Dr. Ray Bard) implied that the initial fractions eluted off the column were incompletely reduced $\mathrm{H}_{2} \mathrm{TNPP}^{\mathrm{O}}$ (red-purple). Later yellow and purple fractions were pure $\mathrm{H}_{2} \mathrm{TAPP}^{0}$ but yielded slightly different spectra. These were normalized when diluted or treated with base. These abnormal spectra appeared to be due either to acidic solvent due to lightcatalyzed decomposition of $\mathrm{CHCl}_{3}$ to $\mathrm{HCl}$ (see Figure 11), or to the presence of aggregated porphyrin.

Purification of HIAPP ${ }^{0}$ by sublimation. I was able to obtain very pure product via sublimation of $\mathrm{H}_{2} \mathrm{TAPP}^{0}$ under high vacuum. However this porphyrin decomposed at temperatures close to its sublimation temperature of $400^{\circ} \mathrm{C}$, resulting in very low yields (2\%). Vacuums higher than $10^{-5}$ Torr would be necessary to make this method of purification practical.

Purification of Commercial Viologens

Many authors report using commercial viologens without purification. However, we have not been able to obtain viologens free of colored impurities from chemical suppliers.

Purification of Commercial $\mathrm{MV}^{2+}$. $\mathrm{MV}^{2+}$ was purchased from Pfaltz and 
Bauer $(98 \%)$ as the chloride salt and recrystallized to remove the yellow contaminant. The ${ }^{1} \mathrm{H}$ NMR before recrystallization is shown in Figure 14. When the sample was recrystallized under room lights the concentration of the yellow contaminant increased with each successive recrystallization as judged from the appearance of the product. Therefore, the sample was protected from room light and oxygen by performing the purification under an argon or nitrogen atmosphere in a glove bag, using only a safelight for illumination. The sample was first treated with decolorizing charcoal as it was found that the colored impurity recrystallized with sample. Three successive recrystallizations from $95 \%$ ethanol were performed. The sample was dried in a vacuum oven in the dark under nitrogen to remove water: 12 hours at room temperature followed by ca. 8 hours at $40^{\circ} \mathrm{C}$. Until use the sample was stored in a vacuum desiccator under nitrogen and protected from light. The sample was checked for signs of organic impurities or decomposition products by UV/VIS absorbance spectroscopy; the NMR spectra of both the monosubstituted $\mathbf{M V}^{1+}$ synthesized by the author following the method described by Braun and coworkers (93), and the disubstituted $\mathrm{MV}^{2+}$ were shown for the purpose of comparison in Chapter II, Figure 14.

Glassware Contamination. The recrystallization described above was performed several times. One 4 gram batch, when tested following recrystallization, was found to be contaminated with a small amount of $\mathrm{H}_{2} \mathrm{TCPP}^{4-}$ which was easily recognized in the absorbance spectrum of concentrated $\mathrm{MV}^{2+}$ as a peak at approximately $420 \mathrm{~nm}$. This was confirmed as the porphyrin peak since it grew upon the addition of an aliquot of $\mathrm{H}_{2} \mathrm{TCPP}^{4-}$. The source of this contamination was traced to the sintered glass filters and points to a flaw in our glassware processing. This problem was solved by rinsing the glassware with dilute base before use to dissolve residual adsorbed anionic tetraphenylporphyrin. 
Synthesis and Purification of PVs

A plausible mechanism for the synthesis of PVs ${ }^{0}$ from $4,4^{\prime}$-bipyridyl and 1,3-propanesultone is illustrated in Figure 18. There are

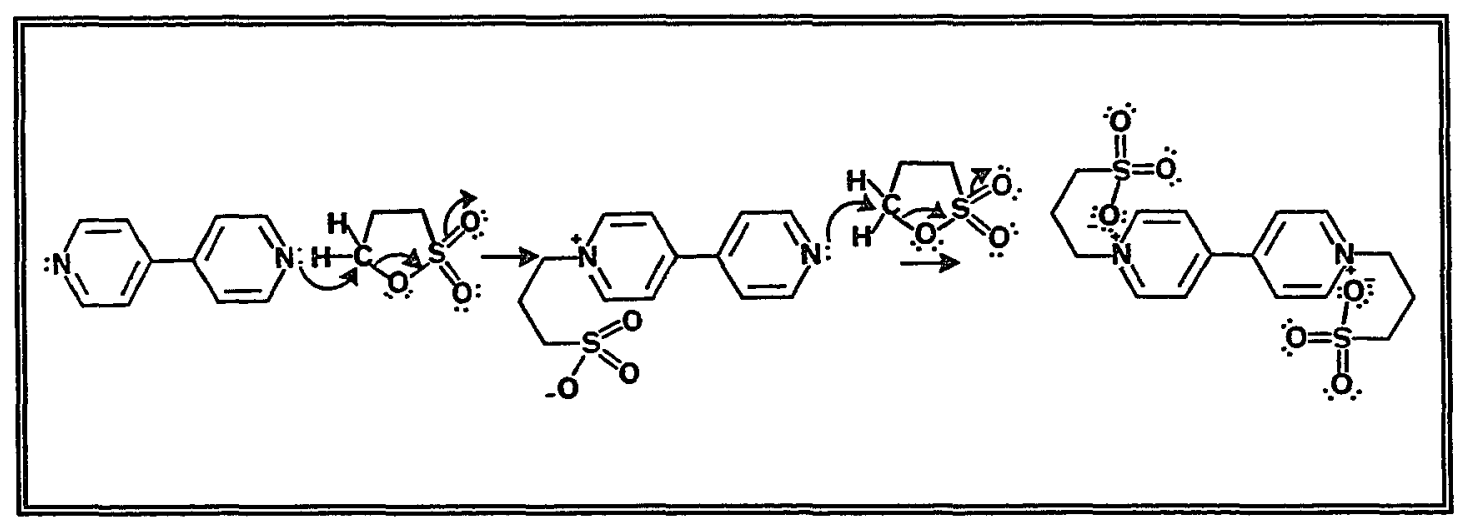

\section{Figure 18. Plausible mechanism for the synthesis of PVs ${ }^{0}$.}

several published methods for the synthesis of $\operatorname{PVs}^{0}(94,97,100,102)$. In my experience the method published by Willner and Degani $(94$,$) yielded$ monosubstituted product. Their protocol described performing the synthesis in acetone (volume of acetone not reported); the monosubstituted product precipitated in acetone, effectively stopping the reaction at this step. Another problem encountered was that any water in the acetone resulted in the hydrolysis of the sultone. The sultone hydrolysis products "oiled out" trapping much of the product in the oil. I was able to extract pure monosubstituted PVs $^{0}$ from the oil into acetonitrile. The $90 \mathrm{MHz}{ }^{1} \mathrm{H}$ NMR spectrum of this compound was shown in Chapter II, Figure 15.

For numerous reasons the use of DMSO as solvent (102) was avoided. In addition to the obvious toxicological problems of handling the severe poisons (sultone and viologen product) in a solvent which readily permeates most laboratory gloves in approximately 5 seconds, DMso is very difficult to remove from the product. We describe a modified version of the method recommended by Ford and Tollin (100) which minimized exposure to the toxins, yielded very pure product and was 
extremely dependable.

Synthesis of PVs $^{0}$. We have obtained the best yields from highly purified bipyridyl. Six successive recrystallizations of 4,4'-bipyridyl (Aldrich, 98\%) from deionized water yielded white needles which, after drying overnight in a vacuum oven gave a sharp melting point at $114{ }^{\circ} \mathrm{C}$ which is the melting point of the anhydrous product. (I found that bipyridyl sublimes in the vacuum oven at temperatures above approximately $\left.30^{\circ} \mathrm{C}.\right)$ The product was stored in a desiccator and protected from light until use.

The synthesis and purification were performed in a glove bag under argon with illumination from a safelight. A dewar half-filled with liquid nitrogen was used as a dehydrating agent, as water vapor freezes on the inner surface of the dewar. The reaction was performed neat, in a $250 \mathrm{ml}$ round-bottom flask fitted with a mechanical stirrer (glass), and immersed in an oil-bath heated to $50{ }^{\circ} \mathrm{C}$ : 4.270 grams of $4,4^{\prime}-$ bipyridyl was slowly added to $25 \mathrm{ml}$ of freshly opened 1,3-propanesultone (Aldrich, gold label). The mole ratio of sultone to bipyridyl was 10.6:1. The mixture was allowed to react for two hours at between 50-70 ${ }^{\circ} \mathrm{C}$, then allowed to cool to room temperature.

The product was collected on a sintered glass filter and washed with dry acetone to remove the unreacted sultone. The product was dissolved in water and precipitated with acetone two times and dried at ambient temperature.

Purification of Pvs $^{0}$. The product was recrystallized and dried under vacuum following the protocol previously described for $\mathrm{Mv}^{2+}$. The average yield was ca. 6.34 grams of white precipitate (58\% yield).

Characterization of PVS $^{0}$. I attempted to ascertain the melting point in a sealed capillary; however, the product behaved in an unusual manner. Before decomposition, several cycles of melting and recrystallization were observed similar to that observed for liquid crystals. It seems reasonable that the product could crystallize in 
several orientations of the propylsulfonate group. Above ca. $250^{\circ} \mathrm{C}$ the product decomposed with the evolution of gas (probably $\mathrm{sO}_{2}$ or $\mathrm{sO}_{3}$ ).

symmetrically substituted bipyridyls yield characteristic ${ }^{1} \mathrm{H}$ NMR spectra; the spectra of the monosubstituted and disubstituted product were shown in Figure 15, Chapter II.

\section{Salts, Buffers, and Detergents}

Buffer. The buffer was exclusively $\mathrm{KH}_{2} \mathrm{PO}_{4} / \mathrm{NaKHPO}_{4}$ at $\mathrm{pH}=7.0$ and was prepared from monobasic potassium phosphate (Aldrich Chemical Company, 99.9998) and adjusted to $\mathrm{pH}=7.0$ with ultrapure sodium hydroxide (Alfa Chemicals).

Inorganic Salts. The chloride salts of the alkali metal ions were all reagent grade.

Organic Salts, Detergents and Crown Ethers. The tetraalkylammonium salts (Southwestern Analytical Chemicals) were dried in the vacuum oven and stored over $\mathrm{P}_{2} \mathrm{O}_{5}$ until use. The detergent, cetyltrimethylammonium bromide (the chloride salt is volatile and toxic) was extracted into petroleum ether twice, dried, and recrystallized twice from ethanol (137). The crown ether, 15-crown-5, was used as received (Aldrich:; its concentration was calcuiated from the density listed in the manufacturer's catalogue.

Organic Solvents. All organic solvents were spectrometric or HPLC grade.

Water. The water was at least distilled and deionized via a commerical system (Corning or Millipore).

\section{INSTRUMENTATION}

\section{UV/VIS Absorbance Spectrophotometer}

All spectra were acquired on a Shimadzu UV260 recording spectrophotometer fitted with extended memory. All titration data were recorded using $0.5 \mathrm{~mm}$ slit width and the monochrometer drive set to the slowest speed (except where noted) allowing for more accurate recording 
of the absorbance near the maxima and more precise positioning of the monochrometer. The spectrophotometer has sufficient memory for the storage of three spectra plus the baseline and simple mathematical capabilities; it will also give a hard copy of the absorbance at six previously specified wavelengths and can run short programs (15 steps). I took advantage of these features during titration experiments where $\mathbf{3 0}$ to 100 spectra at increasing viologen concentrations were acquired, to obtain hard copies of spectra, difference spectra, and the absorbance at six wavelengths for each.

In order to obtain greater than six absorbance values per spectrum, we connected the Shimadzu with a PC-AT microcomputer (80286 with 80287 math coprocessor, 20 megabyte hard drive) via a IEEE-488 interface. The acquisition and data processing software were programmed by Harry Bell.

\section{Nuclear Magnetic Resonance Spectrometers}

90 MHz NMR. The $90 \mathrm{MHz}{ }^{1} \mathrm{H}$ NMR were obtained on a Varian model EM 390 recording NMR using TMS as an external standard.

$300 \mathrm{MHz}$ MMR. The $300 \mathrm{MHz}$ NMR data were obtained on a pulsed GE model QE-300 (Lewis and clark College). The resultant data from 176 scans were processed internally by Fourier transform analysis. Water was used as an internal standard.

\section{Temperature Regulation}

The design of the three temperature regulated cuvette holders will be described in a later section. The temperature of these aluminum heat exchangers was controlled by connecting a Lauda K-2/RD heating/cooling water bath such that the temperature controlled water circulated through the bores within the exchangers. The output rate of the water bath was 1.5 gallons/minute, the temperature stability near room temperature was better than $\pm 0.05{ }^{\circ} \mathrm{C}$ as reported by the manufacturer. 


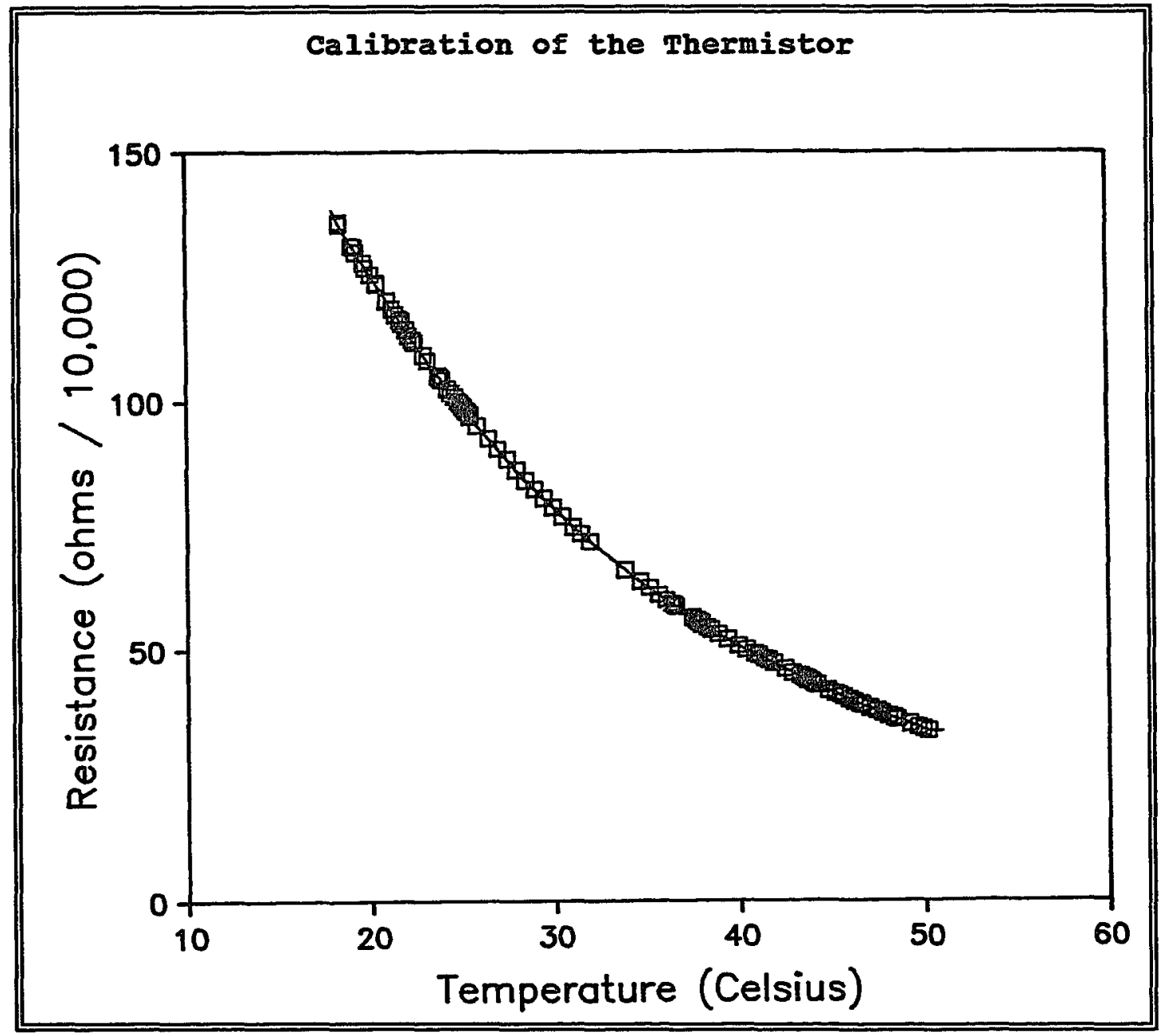

Ficure 19. Thermistor Calibration curve. The points (a) represent the raw data after removal of outliers. The line is the cubic spline fit to the data which was subsequently used as a standard curve to convert $\Omega$ to ${ }^{\circ} \mathrm{C}$.

\section{Temperature Measurement}

The temperature of the solution within the cuvettes was measured with a calibrated thermistor $\left(100 \mathrm{k} \Omega\right.$, nominal $\mathrm{R}$ at $\mathrm{RT}$ of $\left.-4.6 \% /{ }^{\circ} \mathrm{C}\right)$ whose resistance was read on a Hewlett Packard $3490 \mathrm{~A}$ digital output multimeter. The thermistor was calibrated between $19^{\circ} \mathrm{C}$ and $50^{\circ} \mathrm{C}$ using a calibrated thermometer read through a periscope to minimize parallax. The data were fit (Figure 19) using a cubic spline program where $\mathrm{s}=0.3$ (described in a later section), and the calculated curve was used to 
prepare a table relating $\Omega$ to ${ }^{\circ} \mathrm{C}$. The standard deviation of the resistance measurements is thought to be better than $0.07^{\circ} \mathrm{C}$ since standard deviations of the fit using piece-wise quintic polynomials gave this value.

\section{ABSORBANCE MEASUREMENTS: METHODOLOGY}

\section{Introduction}

The effect of a variable such as salt concentration on the UV/VIS spectrum of a porphyrin can be obtained either 1) by the preparation of separate solutions whose spectra are measured individually or 2 ) as a spectrophotometric titration. The advantages and disadvantages of each method are described in Table IV below.

TABLE IV

\section{COMPARISON OF TWO METHODS USED IN THE ABSORBANCE STUDIES}

\begin{tabular}{||c|l|l||}
\hline Method & \multicolumn{1}{|c|}{$\begin{array}{c}\text { Individual ly Prepared } \\
\text { Solutions }\end{array}$} & \multicolumn{1}{|c|}{ Spectrophotometric Titrations } \\
\hline Advantages & $\begin{array}{l}\text { 1. The error in the concentration of } \\
\text { titrant and solution is random rather } \\
\text { than systematic. This means that the } \\
\text { error in concentration is decreased } \\
\text { with the increasing number of data } \\
\text { points measured. }\end{array}$ & $\begin{array}{l}\text { 1. Precision is high as random error } \\
\text { 2. Error associated with cuvette } \\
\text { repositioning is el iminated. } \\
\text { 3. Convenient as titration is rapid } \\
\text { and solutions are al ready thermally } \\
\text { equilibrated. }\end{array}$ \\
\hline Disadvantages & $\begin{array}{l}\text { 1. Random error introduced as cuvette } \\
\text { must be removed and repositioned. } \\
\text { 2. Random error in porphyrin and } \\
\text { titrant concentration introduced with } \\
\text { the preparation of each solution. } \\
\text { 3. Inconvenient as the experiment is } \\
\text { prolonged by time needed for sample } \\
\text { preparation and thermal equilibration. }\end{array}$ & $\begin{array}{l}\text { 1. Accuracy is lower due to higher } \\
\text { probability of systematic error. }\end{array}$ \\
\hline
\end{tabular}

\section{Stock solutions}

Glassware Preparation. The importance of this subject was learned progressively during the course of this study. The silicate surface of glassware can be viewed as a cation exchanger. As porphyrins are 
powerful tetradentate cation chelators, it was recognized from the begining that all traces of heavy metal ions, particularly zinc, copper, iron and cadmium $(72,73)$ must be absent from the surface of the glass. To accomplish this the glassware was treated with EDTA and rinsed with distilled, deionized (DI) water. The glassware was also treated with base to remove residual traces of anionic porphyrins. During the course of the study two previously unrecognized factors were learned:

1) The cationic porphyrin, $\mathrm{H}_{2} \mathrm{TAPP}^{4+}$, binds strongly to the surface of glassware where it can persist through exposure to alcoholic KOH and basic detergents. When adsorbed at low concentrations onto glass surfaces they are not readily detected. If $\mu \mathrm{M}$ concentrations of this porphyrin are present on the surface of a cuvette where the spectrum of $\mathrm{H}_{2} \mathrm{TCPP}^{4-}$ is being studied, what is observed is the time dependent formation of a new absorbance peak to the red of the $\mathrm{H}_{2} \mathrm{TCPP}^{4-}$ Soret band. We have learned (Chapter IV) that this new band is due to the formation of $\mathrm{H}_{2} \mathrm{TAPP}^{0} / \mathrm{H}_{2} \mathrm{TCPP}^{4-}$ heterodimers. It appears that the association complex formed between these two porphyrins is so stable that the presence of the anionic porphyrin effectively removes $\mathrm{H}_{2}$ TAPP $^{0}$ from the surface of the glass or quartz.

Both the porphyrin and heavy metals can be removed by treatment of all glassware with acid. Thus the cleaning procedure was altered midstudy to a cleaning protocol containing an acid washing step.

2) As was discussed in Chapter $I$, the solubility of $\mathrm{H}_{2} \mathrm{TCPP}^{4-}$ is extremely sensitive to $\mathrm{pH}$ and precipitates if the $\mathrm{pH}$ falls below a value of approximately 5 or 6 . To ensure that the anionic sites were not in the acid form on the glass or quartz cuvette surfaces, the cuvettes were rinsed with dilute ultrapure NaOH followed by DI water. This effectively loads the sites with sodium ions. We now think it possible that the variation in sodium ion concentration from experiment to experiment, due to variations in the number of active sites on the cuvette surfaces, may be responsible for the variation in the values 
determined for the $\mathrm{H}_{2} \mathrm{TCPP}^{4-} / \mathrm{MV}^{2+}$ association constants. This subject is explored further in Chapters IV and V.

H$_{2} \mathrm{TCPP}^{4-}$ Aqueous stock Solutions. $\mathrm{H}_{2} \mathrm{TCPP}^{4-}$ was weighed to at least three significant figures. Contrary to the solubility reported by Hofstra et al. (67), workers in both our lab and that of Dr. Martin Gouterman (121) have observed that $\mathrm{H}_{2} \mathrm{TCPP}^{4-}$ does not dissolve readily once the entrapped organic solvents are removed by volatilization. It was found that the $\mathrm{H}_{2} \mathrm{TCPP}^{4-}$ would dissolve if first exposed to strong base; therefore, a minimal volume of $0.1 \mathrm{M} \mathrm{NaOH}$ was added to the weighed porphyrin before the sample was brought to volume with (DI) water. The final $\mathrm{pH}$ of the $100 \mu \mathrm{M}$ stock solution was 10.2 . The sample was prepared under illumination of a safelight and stored in the dark. The stock solution was found to be stable for months as judged by the unchanged absorptivity of the soret band at $414.2 \mathrm{~nm}$ after dilution to a concentration of $1 \mu \mathrm{M}$.

B $_{2}$ ISPP $^{4-}$ Aqueous Stock Solutions. The sodium salt was weighed to at least three significant figures and adjusted to the desired volume with DI water. The solution was prepared under illumination of a safelight and stored in the dark.

Viologen Stock Solutions. The viologens were weighed to 5 significant figures and dissolved in DI water in a volumetric flask. The concentration was either calculated from the weight and volume or from the published absorptivity (96) as either method yielded the same value to three significant figures. The sample was weighed and dissolved under a safelight. All viologen stock solutions were used within 24 hours.

Phosphate Buffer Stock Solutions. Anhydrous monopotassium phosphate was weighed to 5 significant figures, dissolved in DI water, and the $\mathrm{pH}$ adjusted to 7.0 with NaOH using an Orion Research (model 811) digital pH meter which had been calibrated with commercial pH standard solution. After adjusting the buffer to the desired final volume in a 
volumetric flask, the solution was passed through a $0.45 \mu \mathrm{m}$ Millipore filter into a sterile serum bottle to avoid bacterial contamination.

Alkali Metal Salt Stock Solutions. The chloride salts of the alkali metal ions were weighed to a minimum of 5 significant figures and dissolved in a minimal volume of DI water. Contaminating heavy metal salts were removed by passing the solutions through a chelex 100 column (Bio-Rad Laboratory) prior to bringing the solutions to the desired volume in a volumetric flask.

\section{Method 1. Individually Prepared Solutions}

All phases of the experiments described below were performed under the illumination of a safelight.

Solution Preparation. Solutions were prepared in volumetric flasks by dilution of stock solutions and equilibrated to the desired temperature in a dewar in line with the circulating water bath. All absorbance measurements were made within 3 hours after solution preparation.

Absorbance Measurements. The absorbance was measured in order of increasing $\mathrm{H}_{2} \mathrm{TCPP}^{4-}$ concentration in the appropriate cuvette lof pathlengths $5,2,1,0.1$, or $0.05 \mathrm{~cm}$ ) which yielded an absorbance between 0.1 to 1.0. Before each measurement the cuvette was rinsed two times with the solution to be measured. After filling and placement of the cuvette into the instrument the temperature of the solution was monitored with a thermistor until thermal equilibrium was reached before the absorbance spectrum was taken.

Heat Exchanger for Variable Pathlength Cells. Figure 20 illustrates the anodized aluminum block, designed by the author and built in-house by Garo Arakelyan, used to control the temperature of the sample during absorbance measurements. The anodized block was slotted to fit two types of cell adapters: 1) adapters with a rectangular slot which accommodate cells of width of approximately $1 \mathrm{~cm}$ and pathlengths up to $10 \mathrm{~cm}$ and 2) curved bore adaptors which accommodate cylindrical 
cells with pathlengths up to $10 \mathrm{~cm}$. In order to prevent light scattering from the curved edge of the cylindrical cells a mask was fitted to the front surface of each adaptor.

The block was positioned such that the sample cuvette was centered in the light beam. The slot for the reference cuvette adapters was machined wide to allow centering of the adaptor in the reference beam.After optimal positioning the reference adaptor was secured with the aid of metal shims.

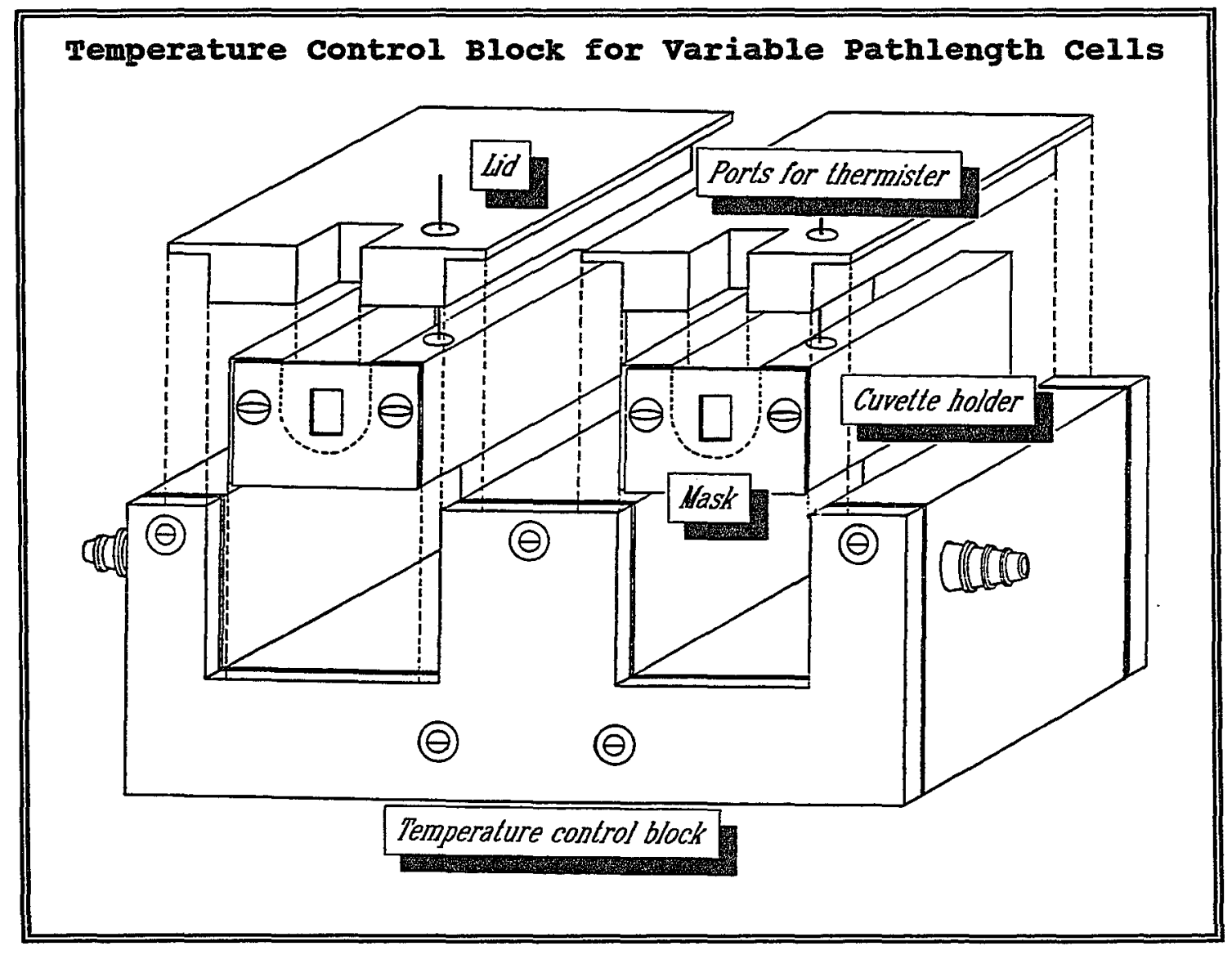

\section{Figure 20. Diagram of Heat Exchanger for Variable Pathlength Cells.}

An anodized aluminum cover prevents thermal equilibrium with the environment. The lid was bored to allow passage of the thermistor through the cover and into the port in the cuvette. 


\section{Method 2. Spectrophotometric Titrations}

One technical problem encountered when using the method of titration is that each incremental addition of titrant results in dilution of the concentration of the solution in the cuvette. To elaborate, in any experiment one component is chosen as the "variable" while, ideally, the concentrations of all other components in the solution remain constant. In this way the effect of each variable can be studied independently; this premise is not valid if the concentration of the "constants" are, in fact, not constant because of dilution. The problem of dilution of the sample with each addition of titrant is usually handled by using the sample volume incremental increase to determine a correction factor which is used to normalize the absorbance value. The correction process necessarily makes assumptions concerning the solution behavior of the components, a situation which the author considers unwise.

In order to avoid the correction process, the titrant was prepared with the same concentration as the "constant" species, so that no correction for dilution was necessary. The method is summarized in Table vI. This table can also be used to describe all titration experiments performed in this study. The following paragraphs give information concerning the methodology in addition to an overview of the types of experiments performed.

Four categories of experiments are defined and can be grouped into two sets. The first set ("A" and "B") describe the study of the effect of salts on the porphyrin absorptivity. "A" defines the "Beer's law" experiments, where the porphyrin concentration is variable. (In order to simplify the table, $\mathrm{pH}$ and temperature are omitted.) What is emphasized is that the concentration of the salt in the buret (Gilmont ultraprecision micrometer buret) was matched to the concentration in the cuvette such that the only variables are the porphyrin concentration and the ionic strength. 
Type "B" is analogous to "A" except that the porphyrin concentration is constant and the salt concentration and ionic strength vary.

The second set ("C" and "D") describes the titration of porphyrin with viologen. The data from these experiments were used to calculate the porphyrin:viologen association constants. The difference between

TABLE $\mathbf{V}$

SPECTRORHOTOMETRIC TITRATIONS: [BURET] VERSUS [CUVETPE]

\begin{tabular}{|c|c|c|c|c|c|}
\hline & & \multicolumn{4}{|c|}{ 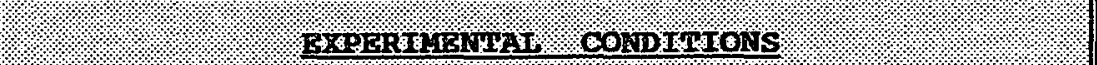 } \\
\hline & & I. & B. & c & D \\
\hline \multirow[t]{5}{*}{$g / 2=1$} & PopPIRTI & high & $\begin{array}{l}\text { matched to } \\
\text { curette }\end{array}$ & $\begin{array}{l}\text { matched } \\
\text { to } \\
\text { cuvette }\end{array}$ & matched to cuvette \\
\hline & GWFE & matched to cuvette & high or $5 \mathrm{~mm}$ & $5 \mathrm{~mm}$ & $5 \mathrm{mM}$ \\
\hline & S11. & absent or present & zero or high & zero & zero \\
\hline & No10es & zero & zero & high & high \\
\hline & $\begin{array}{l}\text { TOHOH } \\
\text { STRETH }\end{array}$ & $\begin{array}{c}\text { depends upon the } \\
\text { experiment, low to } \\
\text { high }\end{array}$ & $\begin{array}{l}\text { depends upon } \\
\text { experiment, } \\
\text { low to high }\end{array}$ & high & calculated as $I_{V}+I_{\text {buffer }}$ \\
\hline \multirow{5}{*}{ WY ATE } & popporirin & variable & constant & constant & constant \\
\hline & BUFFE & constant & $\begin{array}{l}5 \mathrm{~m} \text { or } \\
\text { variable }\end{array}$ & constant & $\begin{array}{l}5 \mathrm{mH} \text { or variable if chosen to } \\
\text { maintain I }\end{array}$ \\
\hline & satr & constant or zero & $\begin{array}{l}\text { zero or } \\
\text { variable }\end{array}$ & zero & $\begin{array}{l}\text { zero or variable if chosen } \\
\text { to maintain I }\end{array}$ \\
\hline & yiotoser & zero & zero & variable & variable \\
\hline & STrREGTH & variable & variable & variable & $\begin{array}{c}\text { constant } I=I_{\text {buffer }}+I_{V} \\
\text { or I } I=I_{\text {salt }}+I_{\text {buffer }}+I_{V}\end{array}$ \\
\hline
\end{tabular}

$\mathbf{P}=$ porphyrin; $\mathbf{V}=$ viologen; $I=$ ionic strength $\left(\xi_{\Sigma} \cdot \sum c_{i} \cdot z_{i}^{2}\right.$, where $c_{i}$ is the ith component concentration and $z_{i}$ is the ith charge integer)

the two columns is that in " $C$ " the variables are viologen concentration 
and ionic strength as opposed to " $D$ " where the variables are viologen concentration and salt (or buffer) concentration. Note that if the ionic strength is constant, the salt or buffer concentration varies. If the salt or buffer concentration is held constant the ionic strength must consequently vary. There is no way to vary the viologen concentration (if the viologen is $\mathrm{MV}^{2+}$ ) and hold both the ionic strength and salt concentration constant. The zwitterionic viologen, PVs $^{0}$, is an exception since the addition of this compound does not affect the ionic strength. We address this subject in detail in Chapters IV and V.

Solution Preparation. The porphyrin solutions were prepared by dilution of the $100 \mu \mathrm{M}$ stock solutions; the accuracy of the final dilutions was increased by using a micrometer buret to dispense the porphyrin stock solutions. All solutions were prepared in volumetric flasks. The solutions were adjusted to the desired volume with distilled deionized water which had been filtered through a $0.45 \mu \mathrm{m}$ Millipore filter to remove particulates which interfere with absorbance measurements.

A known volume of solution was dispensed into the cuvette via: 1) a calibrated volumetric pipette ( $25 \mathrm{ml}$ into a $2 \mathrm{~cm}$ pathlength cuvette), or 2) a micrometer buret (up to $3 \mathrm{ml}$ into a $1 \mathrm{~cm}$ pathlength cuvette). The bageline was run versus DI water in the reference cuvette.

All solutions were used within 5 hours of preparation unless otherwise stated. The porphyrin and viologen solutions were prepared under the illumination of a safelight to avoid photodecomposition.

Solution Preparation Type "A" (Constant Salt, Variable Porphyrin).

Cuvette: Contains buffer, and/or salt solutions. The concentrations and constituents are defined in the specific experiments whose results are presented in Chapter IV.

Ruret: Contains porphyrin, buffer and/or salt solutions. The concentration of the salts and/or buffer are exactly matched to the cuvette. 
Solution Preparation Type "B" (Constant Porphyrin, Variable Salt). Cuvette: Contains porphyrin solution. The concentrations and constituents (buffer, salt, $\mathrm{pH}$, etc.) are defined in the specific experiments whose results are presented in Chapter IV. Buret: Contains concentrated salt solution plus porphyrin whose concentration is exactly matched to the cuvette. Solution Preparation Type "c" (Constant Porphyrin, Variable Viologen, Variable Ionic Strength).

Cuvette: Contains porphyrin solution buffered with $5 \mathrm{mM}$ $\mathrm{KH}_{2} \mathrm{PO}_{4} / \mathrm{NaKHPO}_{4}$.

Buret: Contains concentrated viologen solution. In addition, the solution contains porphyrin and buffer whose concentrations are exactly matched to the concentrations in the cuvette.

Solution Preparation Type "D" (Constant Porphyrin, Constant Ionic Strength, Variable viologen, Variable salt or Buffer).

Cuvette: Contains buffered porphyrin solution whose ionic strength is exactly matched to the viologen solution in the buret with either buffer $\left(\mathrm{KH}_{2} \mathrm{PO}_{4} / \mathrm{NaKHPO}_{4}\right)$ or the chloride salt of an alkali metal ion.

Buret: Contains concentrated viologen. In addition, the solution contains porphyrin and buffer whose concentrations are exactly matched to the concentrations in the cuvette.

Absorbance Measurements. The absorbance was zeroed electronically in the wavelength range to be measured during the titration experiment with filtered DI water in the sample and reference cuvettes. The sample cuvette was then dried, positioned in the instrument, and filled as described above. The sample was allowed to come to thermal equilibrium before the first spectrum was taken. Aliquots of titrant were added by the following procedure:

1) The stirring motor was turned off.

2) The buret tip was lowered a predetermined amount such that the 
tip protruded just under the surface of liquid in the cuvette.

3) A measured amount of titrant was added.

4) The buret tip was raised.

5) The stirring was resumed for 1-2 minutes to allow mixing of the added solution and for chemical equilibrium to be reached.

6) The spectrum was taken.

Control experiments confirmed the chemical stability of porphyrin over this period of exposure to the light beam and the ability of the sample to reach chemical equilibrium during the cycling period between titrant additions.

In a typical titration experiment, 30-100 spectra were taken, each representing a different concentration of titrant. Each spectrum required 5 to 6 minutes for the instrument to run the spectrum depending upon the wavelength range studied. The absorbance data were acquired either as a hard copy by running an internal spectrophotometer program or by the microcomputer through an IEEE-488 interface.

Heat Exchanger for Stirred $2.2 \mathrm{~cm} \times 4.2 \mathrm{~cm}$ Cell. The cell holder consists of an anodized aluminum block with channels to permit circulation of the temperature controlled water bath. Two circular ports allow passage of the light beam through a central rectangular chamber which accomodates a $30 \mathrm{ml}(5.1 \mathrm{~cm} \times 2.2 \mathrm{~cm} \times 4.2 \mathrm{~cm})$ quartz cuvette. The mechanism has a connected double-lid system; an inner Teflon lid is spring-loaded to fit tightly over the cuvette, while an outer metal lid bolts to the block. The glass propeller-type stirrer and the glass tip of the Gilmont ultra-precision micrometer buret enter the chamber through two cylindrical Teflon ports and emerge on either side of the light beam. Thus the sample is in contact with only Teflon or glass and can be stirred without interfering with the measurement of the absorbance. Moreover, aliquots of titrant can be added to the solution in the cuvette without disturbing the cuvette. Figure 21 shows a diagram of this apparatus which was generously loaned by Dr. Dennis 


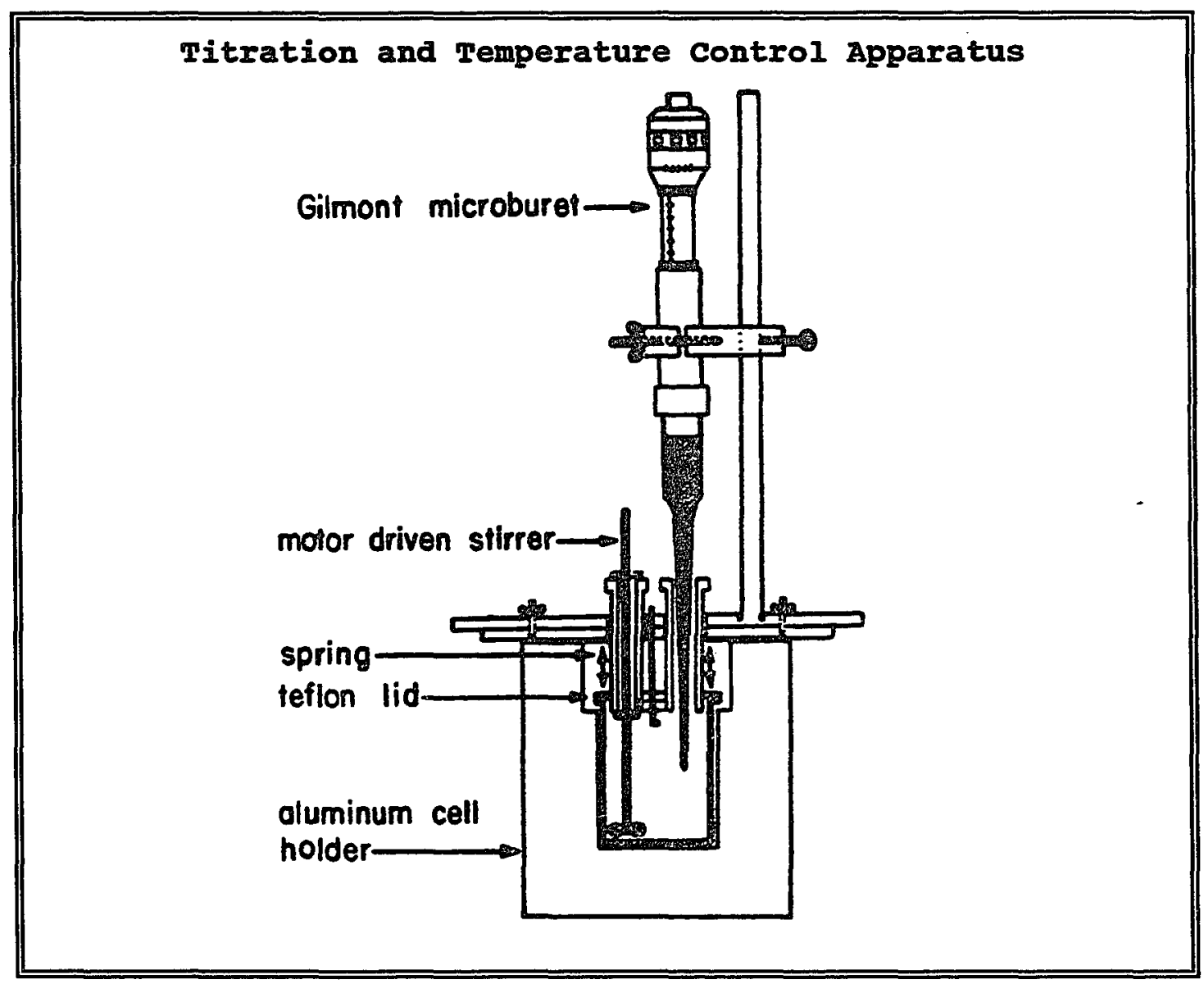

Figure 21. Diagram of the titration apparatus which inserts into the spectrophotometer.

Barnum.

Eeat Exchanger for stirred $1 \mathrm{~cm} \times 1 \mathrm{~cm}$ Cells. The temperaturecontrolled, magnetically stirred cuvette holder supplied with the Spex Fluorolog 2 fluorometer was adapted to fit into the sample chamber of the Shimadzu UV/VIS spectrometer. The temperature was controlled by connecting the cell holder to the Lauda circulating heating/cooling water bath.

A quartz fluorometer cell with a circular small bore top fitted with a Teflon stopper was adapted for the titration experiments by precision drilling three holes through the stopper, one of the precise 
size to allow passage of capillary tubing. The micrometer buret is supplied with an alternate glass tip with a syringe-type tip which fits an adapter connected to capillary tubing. Thus titrant can be added through the tubing (the other ports allow entrance and exit of an inert gas). The cell is stirred by placing a Teflon-coated magnetic stir bar into the cuvette.

\section{MUCLEAR MAGNETIC RESONANCE MEASUREMENTS: METHODOLOGY}

\section{Preparation of solutions}

Chemical Identification. The NMR spectra used for confirmation of the chemical structure of synthesized viologens were obtained by dissolving an unknown quantity of sample in deuterated solvent and taking its NMR spectrum using an external TMS standard. The peaks were integrated to confirm the chemical structure. This technique was critical as viologens have nearly identical UV/VIS spectra but characteristic NMR spectra.

Titration of $\mathrm{MV}^{2+}$ with $\mathrm{H}_{2} \mathrm{TCPP}^{4-} \cdot \mathrm{KD}_{2} \mathrm{PO}_{4} / \mathrm{NaKDPO}_{4}$ was prepared from $\mathrm{KH}_{2} \mathrm{PO}_{4} / \mathrm{NaKHPO}_{4}$ by lyophilizing $5.00 \mathrm{ml}$ of $0.0500 \mathrm{M}$ phosphate $(\mathrm{pH}=7.0$ ) and redissolving in $3.00 \mathrm{ml}$ of freshly opened $\mathrm{D}_{2} \mathrm{O}$. The procedure was repeated before dissolving the sample ( 4 hours before the experiment) in $1.00 \mathrm{ml}$ of $\mathrm{D}_{2} \mathrm{O}$ to yield a final $\mathrm{KD}_{2} \mathrm{PO}_{4} / \mathrm{NaKDPO}_{4}$ concentration of $0.250 \mathrm{M}$. The $\mathrm{PD}$ was tested after the experiment and found to equal $7 \pm 0.2 \mathrm{pH}$ units.

A $0.625 \mathrm{mM} \mathrm{H} \mathrm{HCPP}^{4-}$ stock solution in $\mathrm{D}_{2} \mathrm{O}$ was prepared by lyophilizing $18.75 \mathrm{ml}$ of $0.100 \mathrm{mM} \mathrm{H}_{2} \mathrm{TCPP}^{4-}$ stock solution. The sample was redissolved in $3.00 \mathrm{ml}$ of freshly opened $\mathrm{D}_{2} \mathrm{O}$ and the process was repeated. Four hours before the experiment the porphyrin was dissolved in $3.00 \mathrm{ml}$ of freshly opened $\mathrm{D}_{2} \mathrm{O}$.

The dried viologen was weighed $(0.1285 \mathrm{~g})$ into a $10 \mathrm{ml}$ volumetric flask and stored in a desiccator until dissolving in $D_{2} \mathrm{O} 4$ hours before the experiment. The final concentration of the viologen stock solution 
was $50.0 \mathrm{mM}$.

The solutions to be measured were prepared by dilution of the stock solutions with $\mathrm{D}_{2} \mathrm{O}$ in a $2.0 \mathrm{ml}$ volumetric flask minutes before their NMR spectra were taken.

DATA ANALYSIS

Cubic Spline Calibration Curve for Thermistor Temperature Measurements

The data were smoothed using a cubic spline program (138) that incorporates measurement error by means of a smoothing parameter, $S$, that reflects the global smoothness-accuracy tradeoff. A value for $s$ was found by inspection to provide a sufficiently uncorrelated distribution of residuals while maintaining a relatively non-oscillating curve.

Determination of Solution Parameters by Least-Squares Methods

Linear Least-squares. The infinite dilution Beer's Law absorptivity was estimated using linear regression on the set of data which gave the highest correlation coefficient $\left(r^{2}\right)$. This was determined by systematically increasing the number of low concentration data used until a maximum $r^{2}$ value was found.

Nonlinear Ieast-Squares Fitting to Gaussians. The decomposition of spectral line shapes into Gaussian curves is often of intrinsic interest. We found that three Gaussians and a linear component could generally fit the soret spectral region satisfactorily.

We used the Marquardt-Levenburg algorithm as implemented by Press, et al. in Numerical Recipes ((138) their sample test function happens to be a linear combination of Gaussians). We found it necessary to include a linear component which could be interpreted as either instrumental drift and/or an approximation of the tail of a very large Gaussian.

It was found to be very important to obtain fairly good estimates of the Gaussian parameters in order for the algorithm to "lock in" to a solution. 
Nonlinear Least-Squares. Provided the self-aggregation of TCPP is insignificant we can model the solution equilibria in terms of the two reactions (the charges are omitted for simplicity):

$$
\begin{aligned}
\mathrm{TCPP}+\mathrm{MV} & =\mathrm{TCPP}-\mathrm{MV} \\
\mathrm{TCPP}-\mathrm{MV}+\mathrm{MV} & \neq \mathrm{MV}-\mathrm{TCPP}-\mathrm{MV}
\end{aligned}
$$

with equilibrium constants $k_{1}$ and $k_{2}$ respectively.

For notational convenience let us now define the following:

$$
\begin{aligned}
\mathbf{A} & =[\mathrm{TCPP}] \\
\mathbf{B} & =[\mathrm{MV}] \\
\mathbf{C} & =[\mathrm{TCPP}-\mathrm{MV}] \\
\mathbf{D} & =[\mathrm{MV}-\mathrm{TCPP}-\mathrm{MV}] \\
\mathbf{A}_{\text {ex }} & =\text { Absorptivity of TCPP } \\
\mathbf{C}_{\text {ex }} & =\text { Absorptivity of TCPP-MV } \\
\mathbf{D}_{\text {ex }} & =\text { Absorptivity of MV-TCPP-MV } \\
\mathbf{A}_{\mathbf{t}} & =\text { Total concentration of TCPP } \\
\mathbf{B}_{\mathbf{t}} & =\text { Total concentration of MV } \\
\mathbf{Y} & =\text { Absorbance }
\end{aligned}
$$

With the two mass balance equations:

$$
\begin{aligned}
& A_{t}=A+C+D \\
& B_{t}=B+C+2 \cdot D
\end{aligned}
$$

and the two equilibrium equations:

$$
\begin{aligned}
& \mathrm{C}=\mathrm{K}_{1} \cdot \mathrm{A} \cdot \mathrm{B} \\
& \mathrm{D}=\mathrm{K}_{2} \cdot \mathrm{B} \cdot \mathrm{C}
\end{aligned}
$$

solving the above nonlinear system of equations (with unknowns A, B, C, and D) can be accomplished by reduction to a single equation with one unknown, B:

$K_{1} \cdot K_{2} \cdot B^{3}+\left(2 \cdot K_{1} \cdot K_{2} \cdot A_{t}-K_{1} \cdot K_{2} \cdot B_{t}+K_{1}\right) \cdot B^{2}+\left(K_{1} \cdot A_{t}-K_{1} \cdot B_{t}+1\right) \cdot B-B_{t}=0$ The absorbance equation is linear with respect to solution concentration (Beer's Law):

$$
\mathbf{Y}=\mathrm{A}_{\mathrm{ex}} \cdot \mathrm{A}+\mathrm{C}_{\mathrm{ex}} \cdot \mathrm{C}+\mathrm{D}_{\mathrm{ex}} \cdot \mathrm{D}
$$

Hence, the trial and error methodology is as follows:

1. Obtain data for absorbance $\left(Y_{i}\right)$ vs. total concentration of $\mathrm{MV}^{2+}$ $\left(B_{t}\right)$. The absorbance data were sampled near the $\lambda_{\max }$ of the soret band of monomer porphyrin $(414.2 \mathrm{~nm})$ for each of the concentrations used 
(Chapter V, experiment 1); two additional absorbance values near the $\lambda_{\max }$ 's of the two porphyrin/viologen complexes may also have been used (Chapter $V$, experiments 2 and 3). A weighting factor equal to the incremental $\left[\mathrm{MV}^{2+}\right.$ ] between successive concentrations was used in order to even out the uneven sampling with respect to $\mathrm{MV}^{2+}$ concentration.

2. Pick candidate values for the parameters $K_{1}, K_{2}, C_{e x}$ and $D_{\text {ex }}$.

3. Solve the cubic equation above for $B$ followed by solving for $A$, $C$, and $D$.

4. Evaluate the resulting absorbance ( $Y$ ) and compare it with the $\operatorname{data}\left(Y_{i}\right)$.

5. If no other parameter set can be found with a smaller difference between predicted and measured absorbance for all the data in the least-squares sense we consider this set to be the most likely one; otherwise repeat the process from step 2 with a new parameter set.

Uncertainty in the parameters obtained by this method is estimated using a Monte Carlo error analysis technique. In this technique a series of psuedo data sets are constructed based upon measured data and the apparent overall noise level in the data. More precisely, each datum is generated by summing the measured data via a random Gaussian deviate with a standard deviation equal to the measurement noise level. Each psuedo data set is then used in the above nonlinear least-squares procedure to make an independent set of parameter estimates. The uncertainty is then simply estimated as the standard deviation in the resulting parameter estimates for all the psuedo data sets.

Speciation Determination Technique Using Principle Component Analysis

Principal component analysis (PCA) has been used extensively in solution speciation studies (104-112). We present a novel extension of the two-component principal component analysis method of Lawton and Sylvestre (105) to systems in which multiple, disjoint concentration regions are known to have only two components. Such concentration regions can be identified by isosbestic points (wavelengths at which 
absorbance does not change with changing titrant concentrations). Following are the steps, described below and in the flow chart in Figure 22, involved with this speciation technique (abbreviations for this section will be: $P=$ porphyrin, $V=$ viologen; thus $P-V=$ the $1: 1$ and $\mathrm{V}-\mathrm{P}-\mathrm{V}$ the $1: 2$ porphyrin:viologen association complexes):

1) Form a data matrix, A, with rows corresponding to absorbances at different wavelengths and columns corresponding to absorbances at different titrant concentrations (an RxC matrix, where $R=$ number of wavelengths and $\mathbf{c}=$ number of concentrations).

2) Find all eigenvalues, $a_{i}$, and the corresponding eigenvectors, $\mathbf{x}_{\mathbf{i}}$ of the data covariance matrix, $A \cdot \mathrm{A}^{\mathrm{t}}$.

3) Plot $\log \left(a_{i}\right)$ vs. $i$ and note the number of eigenvalues which are substantially larger than the rest. This is the number of components (chemical species which have distinct absorbance spectra). In the $\mathrm{P}-\mathrm{V}$ titrations we found three substantial components.

4) Find groups of concentrations in which the absorbance does not change at some particular wavelength with changing concentration (isosbestic points). Repeat steps 1-3 for each of these new reduced data matrices (where A will now have a number of columns equal to the number of spectra passing through the isosbestic point). There should be two components (i.e. two substantially larger eigenvalues) for each isosbestic point. Retain the eigenvectors corresponding to the largest two eigenvalues for each isosbestic point.

5) We model the system as having three components: P, P-V, and VP-V. With this assumption, as the titration proceeds (concentration of $\mathrm{V}$ increases) we can expect the number of components present to be, in succession: one $(P)$, two $(P$ and $P-V)$, three $(P, P-V$, and $V-P-V)$, two ( $P-$ $V$ and $V-P-V)$, and finally one $(V-P-V)$. We observe that one of the components, $\mathrm{P}-\mathrm{V}$, is in common with the 2 two-component regions. This fact should give us an added element of perspective in the estimation of its spectral line shape. 
6) We now follow the clearly developed procedures for twocomponent PCA (105) in order to determine the spectral line shapes in the 2 two-component isosbestic regions. In this procedure, a linear combination of the two eigenvectors saved in step 4 is sought for each of the two components such that the resulting two line shapes are nonnegative at all wavelengths. Furthermore, these resulting line shapes must be such that they not require negative coefficients (concentrations) in order to fit the measured absorbances. These requirements are met in what is often represented graphically as two wedge-shaped regions in the two-dimensional space of eigenvector coefficients $(105,108,111)$.

We choose as the spectral line shape for the first component, P, the absorbance spectrum without any viologen added; this absorbance spectrum when divided by the known concentration of $P$ gives its absorptivity.

since the second component line shape of the first isosbestic region should be congruent with the first component line shape of the second isosbestic region we can use this congruency condition to uniquely determine the normalized spectral line shape for $P-V$. In practical terms, there is a free parameter for the second component of the first isosbestic and a free parameter for the first component of the second isosbestic. We choose the pair of parameters which result in the best matching of the two spectral line shapes in the least-squares sense. The $P-V$ absorptivity curve is determined by forcing it to cross the $P$ absorptivity curve at the first isosbestic wavelength point. The V-P-V spectral line shape is less easily obtained. If the second isosbestic point has a clear ending concentration this spectrum can be used directly as its spectral line shape. Otherwise, a most plausible line shape is chosen among those possible within the wedgeshaped region in the space of eigenvalue coefficients $(108,111)$. The VP-V absorptivity is determined such that it crosses the P-V absorptivity 
curve at the second isosbestic wavelength point.

7) Now that the three absorptivity spectra have been determined, solution concentrations can be determined by multivariable linear regression.

8) The equilibrium constants can then be determined from the solution concentrations.

\section{COMPUTER RROGRAMS}

We have omitted listings of the computer programs used in the data analysis but the author can furnish them upon request. The basis for many of the mathematical subroutines (available on diskettes in Fortran or c) can be found in the extremely useful reference, "Numerical Recipes" (138). 


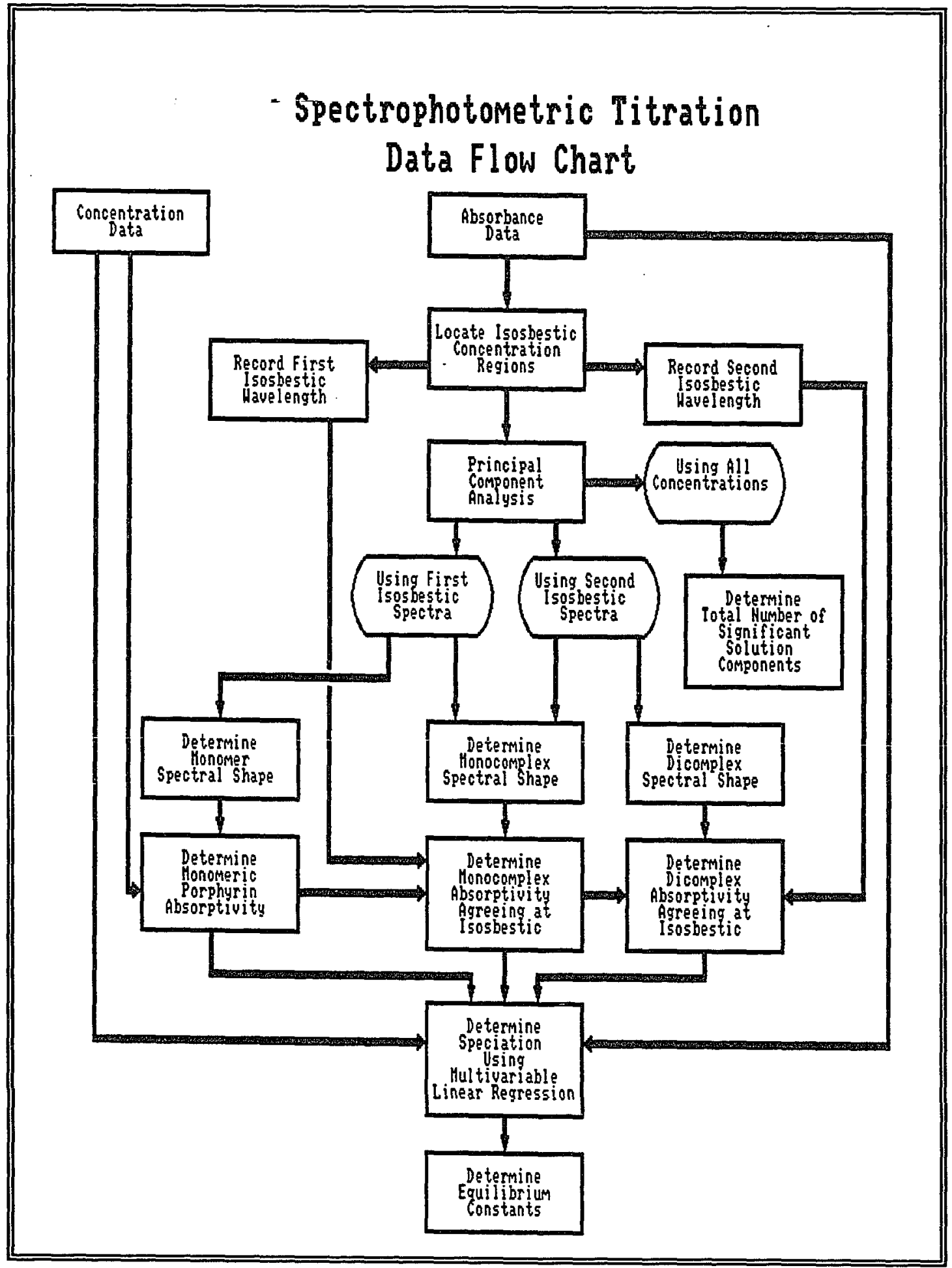

Figure 22. Flow diagram illustrating the steps involved in principal component analysis. 
Chapter IV

THE EFFECI OF SAITS AND SOLVENTS ON THE SPECTRUM AND SPECIATION OF $\mathrm{H}_{2} \mathrm{TCPP}^{\mathrm{O}}\left(\mathrm{H}_{2} \mathrm{TCPP}^{4-}\right.$ )

INTRODUCIION

The goal of this work was the quantification of $\mathrm{H}_{2} \mathrm{TCPP}^{4-} / \mathrm{viologen}$ association constants, a subject covered in Chapter $v$. A preview of the spectral patterns observed in the titrations, along with the corresponding absorbing species in solution, is illustrated in Figure 23. In this chapter we present the control experiments necessary to both define a set of appropriate experimental conditions for determination of the equilibrium constants and to correlate the observed spectral changes with specific porphyrin species in solution.

Each experiment in this chapter was performed in order to clarify some aspect of the titration data of $\mathrm{H}_{2} \mathrm{TCPP}^{4-}$ with either $\mathrm{MV}^{2+}$ or $\mathrm{PVS}^{0}$; this required collecting data concerning the subjects listed below:

1) Both the soret band absorptivity and the wavelength of the Soret maxima of the $\mathrm{H}_{2} \mathrm{TCPP}^{4-}$ monomer were determined.

2) The conditions where only monomer is in solution were determined in order to neglect the dimer in the mathematical analysis of the spectral data used in the calculation of the porphyrin:viologen association constants.

3) We confirmed that the red-shifted and weakened soret band observed upon the addition of viologen was due to the formation of porphyrin/viologen association complexes and not to some other phenomenon such as porphyrin dimerization.

4) Also investigated were the spectrum of the porphyrin dimer and the conditions under which dimerization could be induced. 
The importance of these subjects can be best illustrated by contrasting the titration of $\mathrm{H}_{2} \mathrm{TCPP}^{4-}$ with $\mathrm{MV}^{2+}$ versus the titration of $\mathrm{H}_{2}$ TCPP $^{4-}$ with PVS ${ }^{0}$. In Figure 23 the viologen concentration-dependent $\mathrm{H}_{2}$ TCPP $^{4-}$ spectral changes are presented. The two individual experiments were performed as titrations, where the buffered ( $5 \mathrm{mM}$ phosphate)

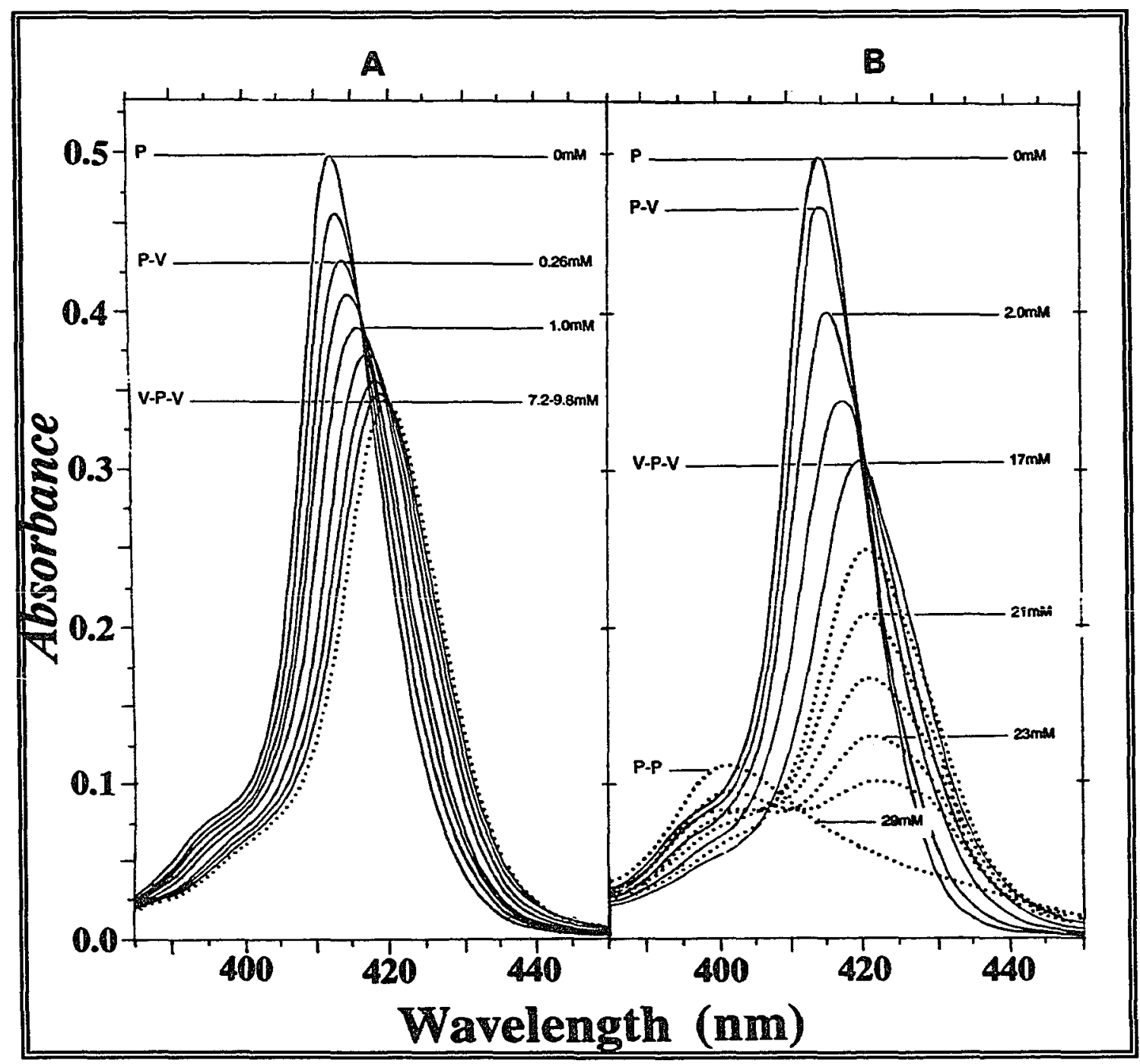

Figure 23. Titration of $\mathrm{H}_{2} \mathrm{TCPP}^{4-}$ with A) $\mathrm{MV}^{2+}$ and B) $\mathrm{PVS}^{0}$. Selected spectra from titrations of $1 \mu \mathrm{M} \mathrm{H}_{2} \mathrm{TCPP}^{4-}$ with $\mathrm{MV}^{2+}$ (50 pts. taken) or Pvs $^{0}$ (120 pts. taken): $\mathrm{pH}^{\mathrm{H}}=7.0$, [phosphate] $=5 \mathrm{mM}, T=20^{\circ} \mathrm{C} . \mathrm{P}, \mathrm{P}-\mathrm{P}, \mathrm{P}-\mathrm{V}$, and $\mathrm{V}-\mathrm{P}-\mathrm{V}$ indicate monomer porphyrin, dimer porphyrin, $1: 1$ and $1: 2$ porphyrin: viologen association complexes, respectively. selected concentrations of titrant are indicated on the graph; "..." indicate high [titrant]. 
$\mathrm{H}_{2} \mathrm{TCPP}^{4-}$ concentration was constant and the viologen concentration varied. In each case the viologen concentration was increased past the point where additional viologen caused no further change in the observed absorbance. Within the lower viologen concentration range the two experiments show similar spectral changes; the porphyrin soret band is progressively red-shifted and weakened with each incremental viologen addition. Within this concentration range $\mathrm{H}_{2} \mathrm{TCPP}^{4-}$ : viologen association complexes with stoichiometries of $1: 1$ and $1: 2$ are observed for both $\mathrm{MV}^{2+}$ and $\mathrm{PVS}^{\mathrm{O}}$ (described in detail in Chapter V).

The two viologens induce dramatically different spectra at higher viologen concentrations. In Figure 23A, the titration of $\mathrm{H}_{2} \mathrm{TCPP}^{4-}$ with $\mathrm{MV}^{2+}$, the final spectrum is consistent with viologen-complexed porphyrin (red-shifted and weakened spectrum) and the spectrum at the viologen concentration where additional aliquots of viologen induce no further changes in the porphyrin absorbance (leveling-off point) is consistent with the complete conversion of monomer porphyrin to viologen-complexed porphyrin. In Figure 23B, the titration of $\mathrm{H}_{2} \mathrm{TCPP}^{4-}$ with $\mathrm{PVS}^{0}$, the final spectrum is consistent with a porphyrin dimer (blue-shifted and severely weakened) and the leveling-off is consistent with the complete conversion of porphyrin monomer to porphyrin dimer (the dimer may or may not be complexed with viologen, the severe blue shift may indicate that it is not).

In order make the interpretations described above, the author had to be able to associate the observed spectral changes with specific solution phenomena. This chapter contains the studies which allowed these correlations. The data complexity required a large number of control experiments; as a result, this chapter contains the most comprehensive study of the behavior of any free base porphyrin in aqueous solution. Consequently, we have discovered several subjects which need to be explored as they question fundamental theories of the behavior of porphyrins (and perhaps the larger subject of the behavior 
of organic dyes) in aqueous solution.

\section{SUMMARY OF THE DATA PRESENTED IN THIS CHAPTER}

This is an overview of the data and conclusions presented in this chapter.

1) $\mathrm{H}_{2} \mathrm{TCPP}^{4-}$ was found to exist in monomer form only below a concentration of $2 \mu \mathrm{M}$ in aqueous solution buffered to $\mathrm{pH}=7$ with $5 \mathrm{mM}$ phosphate buffer at $20^{\circ} \mathrm{C}$. The $\mathrm{H}_{2} \mathrm{TCPP}^{4-}$ monomer soret band has an absorbance maximum at $414.2 \mathrm{~nm}$ and an absorptivity of approximately $480,000 \mathrm{M}^{-1} \mathrm{~cm}^{-1}$. The absorptivity was found to be a function of the alkali metal ion concentration.

2) Porphyrin dimerization was induced by: a) $3 \mathrm{M} \mathrm{NaCl}$ (Figure

28); b) high concentrations of the zwitterionic viologen, PVS ${ }^{0}$ (Figure 23B); c) equimolar concentrations of a nitrogen $8+$ macrocycle, $1,5,9,13,17,21,25,29$-octaazacyclodotricontane $[32]-\mathrm{N}_{8}\left(\mathrm{~N}_{8} \mathrm{H}_{8}{ }^{8+}\right)(69) ;$ d) equimolar concentrations of the porphyrin $\mathrm{H}_{2} \mathrm{TAPP}^{0}$ (or $\mathrm{H}_{2} \mathrm{TAPP}^{4+}$, the exact charge on the porphyrin is not known under the experimental conditions) (Figure 27) $(64,67)$ and e) approximately equimolar concentrations of the cationic tetraalkylammonium detergent cetyltrimethylammonium bromide (CTAB). From these experiments it was learned that the porphyrin dimer has an absorbance maximum at approximately $400 \mathrm{~nm}$ and an absorptivity of approximately $100,000 \mathrm{M}^{-1} \mathrm{~cm}^{-1}$.

3) Under specific conditions defined in each experiment, porphyrin dimer dissociation was induced by: a) methyl viologen (Figure 29), b) the crown ether 15-crown-5 (Figure 28), a) the detergent Crab (Figure 27) and d) inorganic salts (69).

4) Although the effect of organic solvents was not studied comprehensively, it appears that aprotic organic salts and solvents yield a red-shifted porphyrin soret band combined with an absorbance weakening. However, with protic mixed-solvent solutions, under certain 
conditions, we observed a red shift with no loss in absorbance. The red shift is consistent with spectral changes observed for other polar organic chromophores in that less polar solvent environments result in a red-shifted absorbance relative to the maxima observed in water $(126,139)$. The solvent dependent loss in absorptivity is not understood but may indicate association of the salt or solvent with the hydrophobic porphine core, as in the case of the porphyrin absorbance changes observed upon addition of viologen. We would like to find a theoretical basis for this model; unfortunately, the theory concerning the effect of solvent on the absorptivity of a chromophore is not well developed $(70,139)$.

5) The addition of inorganic salts to aqueous solutions of $1 \mu \mathrm{M}$ $\mathrm{H}_{2} \mathrm{TCPP}^{4-}$ resulted in a weakened and broadened soret band. At high concentrations of inorganic salts a blue shift is observed and the soret is substantially weakened, conditions found to be characteristic of the dimer. At $0.5 \mathrm{M} \mathrm{NaCl}$, increased concentration of $\mathrm{H}_{2} \mathrm{TCPP}^{4-}$ into the 0.1 $\mathrm{mM}$ concentration range results in a blue-shifted (approximately $4 \mathrm{~nm}$ ) and weakened Soret but the spectral shape of the monomer is preserved (the high energy shoulder is still present).

THE EFFECT OF ORGANIC SOLVENTS ON THE H $\mathbf{2}^{\text {TCPPO }}$ SPECTRUM

All of the spectra of $\mathrm{H}_{2} \mathrm{TCPP}^{0}$ in organic solvents showed both a red-shifted (from $\approx 414 \mathrm{~nm}$ in water to $417-422 \mathrm{~nm}$ in organic solvents, Chapter II, Figures 4 and 6 ) and weakened soret relative to the spectrum of $\mathrm{H}_{2} \mathrm{TCPP}^{4-}$ in aqueous solution. The absorptivity of $\mathrm{H}_{2} \mathrm{TCPP}^{\mathrm{O}}$ is 400,000 $\mathrm{M}^{-1} \mathrm{~cm}^{-1}$ in THF where the wavelength maximum of the soret band is 417.5 nm.

When the porphyrin is dissolved in different mixtures of ethylene glycol:water, what is observed is a red-shifted but not weakened soret above $50 \%$ ethylene glycol or at lower ethylene glycol concentrations in the presence of NaOH (Figure 24). Below $50 \%$ ethylene glycol or in the 


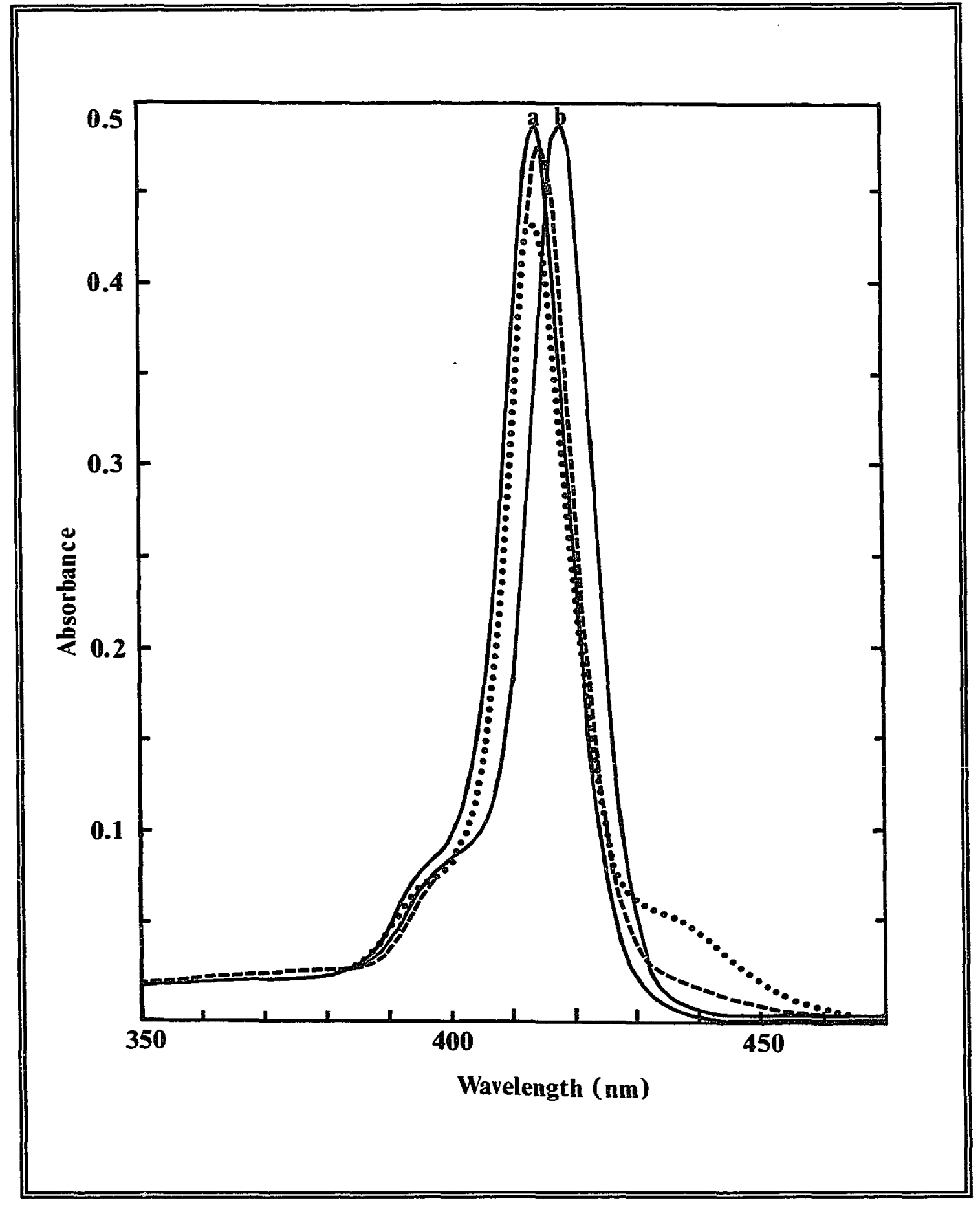

Figure 24. The spectrum of $\mathrm{H}_{2} \mathrm{TCPP}^{4-}$ in different water:ethylene glycol mixtures. The "...." and solid" line "a" show $1.00 \mu \mathrm{M}$ HCPP" in DI water and DI water containing $0.5 \mathrm{mM}$ NaOH, respectively. The line "--" is 108 ethylene glycol (no base) the solid line "b" shows 90\% ethylene glycol (no base). The solutions were prepared by method "1" (Chapter III). 
absence of base the weakening of the soret resembles the spectra observed with other organic solvents. Table VI and Figure 24 show the effect of different ratios of mixed ethylene glycolswater in the presence and absence of base. Notice that the additional peak at higher wavelength is analogous with that observed in Figure 12. Therefore this peak was identified as the monocation in equilibrium with the dication. The results for $\mathrm{H}_{2}$ TSPP $^{4-}$ were similar.

Table VI

THE EFFECT OF ETHYLEME GLYCOL:WATER SOLUTIONS ON THE PORPHYRIN SPECTRUM

\begin{tabular}{|c|c|c|c|c|}
\hline \multirow[t]{2}{*}{$8 \quad E$} & \multicolumn{2}{|c|}{$\begin{array}{c}\text { ethylene glycol:water } \\
\text { (at } 0.5 \mathrm{mM} \text { ) NaOH }\end{array}$} & \multicolumn{2}{|c|}{$\begin{array}{c}\text { ethylene glycol:water (no } \\
\text { base) }\end{array}$} \\
\hline & $\lambda_{\max } \underset{(\mathrm{nm})}{\text { soret }}$ & $\begin{array}{c}\text { Absorptivity } \\
\left(\mathrm{M}^{-1} \mathrm{Cm}^{-1}\right)\end{array}$ & $\lambda_{\max } \underset{(\mathrm{nm})}{\text { soret }}$ & $\begin{array}{l}\text { Absorptivity } \\
\qquad\left(M^{-1} \mathrm{Cm}^{-1}\right)\end{array}$ \\
\hline 0 & 414.0 & 484,000 & 414.0 & 431,000 \\
\hline 10 & 415.0 & 482,000 & 414.9 & 472,000 \\
\hline 50 & 416.7 & 481,000 & 416.6 & 480,000 \\
\hline 90 & & & 418.0 & 488,000 \\
\hline
\end{tabular}

Schmehl and coworkers (90) have observed a similar shift in the monocation f free base equilibrium as a function of the increased concentration of an organic molecule, poly(vinylpyrrolidone) (PVP) in mixed water/PVP solutions. They interpret this as the result of a less acidic porphyrin environment which occurs as a result of porphyrin association with the organic polymer. Ethylene glycol is less acidic than water, thus the increase of ethylene glycol shifts the equilibrium toward the free base.

THE EFFECT OF ORGANIC SALTS ON THE PORPHYRIN SPECTRUM

Two alkylammonium chloride salts were tested in the search for a 
non-interacting salt to maintain the ionic strength in the titration of $\mathrm{H}_{2} \mathrm{TCPP}^{4-}$ with viologens. As can be seen in Figure 25, the addition of

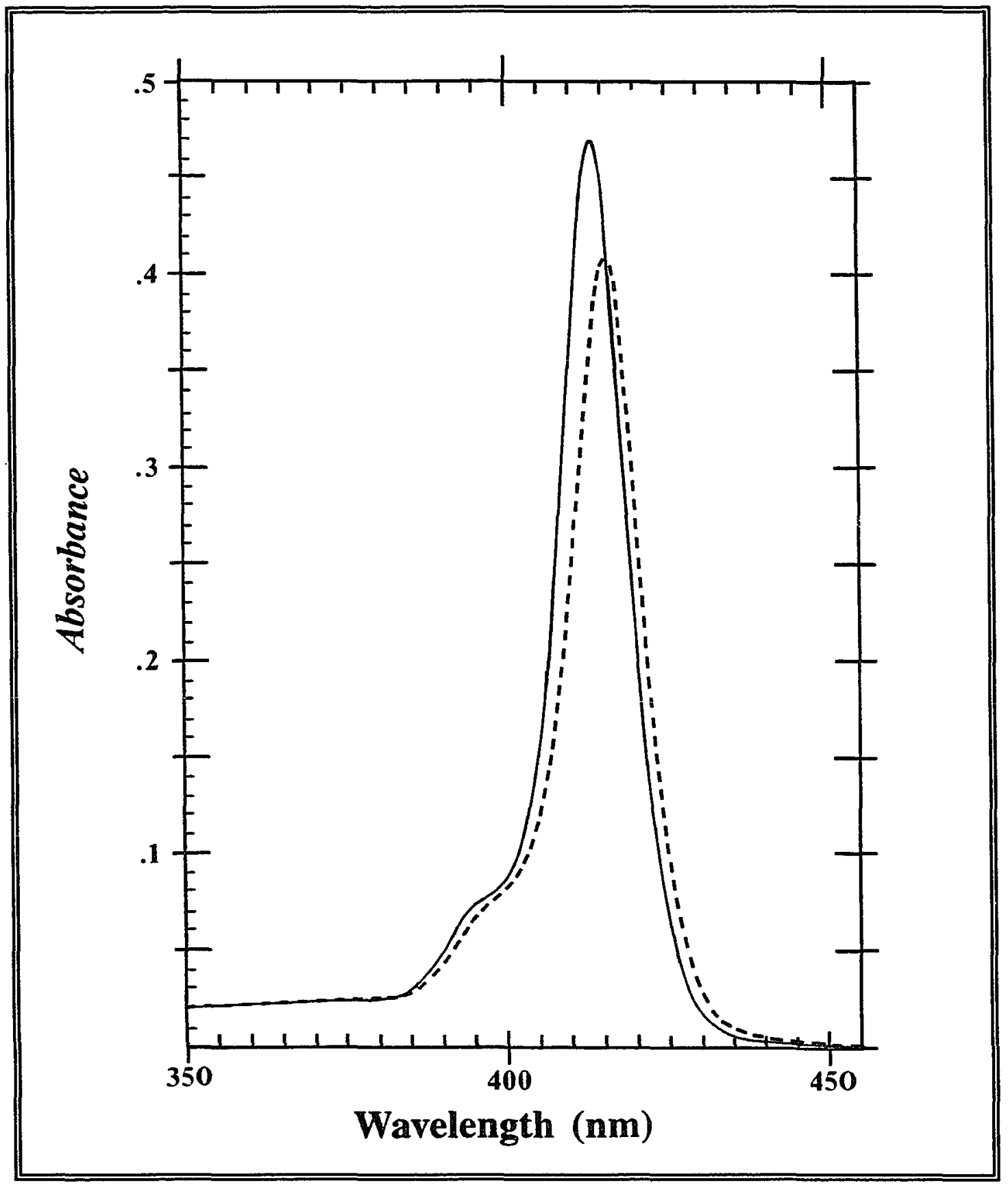

Ficure 25. The effect of tetramethylammonium perchlorate on the spectrum of $1 \mu \mathrm{M} \mathrm{H}_{2} \mathrm{TCPP}^{4-}$. $\mathrm{T}=20^{\circ} \mathrm{C}$, $\mathrm{pH}=7.0^{\circ}$ and [phosphate]=5 mM: (-) porphyrin, (--) porphyrin in tetramethylammonium perchlorate ( $80 \%$ of saturated concentration). 
alkylammonium salts induces spectral changes similar to that observed in Figure 23 with viologens (red-shifted and weakened soret). This has also been observed for ZnTSPP ${ }^{4-}$ by Schmehl and Whitten (44). Since a possible explanation for the loss in absorptivity is the formation of porphyrin/salt association complexes, the tetraalkylammonium salts were not used to maintain the ionic strength in the titration experiments. Method 1 described in the experimental section was used to acquire the data in the figure above. The experiments were more qualitative than quantitative, thus the precise concentration of the salt was less important than the effect of the salt on the porphyrin spectrum. Because the perchlorate salts may be explosive when dry, the author was reluctant to heat the salt to dryness. A saturated solution of the salt was prepared and the concentration is expressed as the percent of the saturated solution. Both the chloride and perchlorate salts of tetramethyl and tetraethylammonium salts were tested and found to yield similar results.

The Effect of Organic Salts on the Induced Dimerization and Dimer Dissociation of the Anionic Tetraphenylporphyrins

In Figure 23 the induced dimerization of $\mathrm{H}_{2} \mathrm{TCPP}^{4-}$ by high concentrations of PVS $^{0}$ was illustrated; in addition, we cited the induced dimerization of $\mathrm{H}_{2} \mathrm{TSPP}^{4-}$ by a cationic nitrogen macrocycle (69). In this section other examples of induced anionic tetraphenylporphyrin dimerization are presented. This phenomenon is not limited to an effect of cationic organic salts on anionic tetraphenylporphyrin solution speciation as will be shown by citing examples from the xanthene dye literature.

Porphyrin dimer spectra are described in the literature by excitonic coupling theory, where the resultant peak is thought to be the allowed transition of the dimer. The theory $(70,122-125)$ predicts that the presence of a second porphyrin will split the absorbance into twice the number of peaks observed in the monomer, one peak being blue-shifted 
and one peak being red-shifted relative to the porphyrin monomer. The absence of a red-shifted peak is explained by assuming that this peak is not allowed. Although the use of excitonic coupling theory to explain the dimer spectrum has not been questioned, there is some controversy concerning its interpretation $(70,123,125)$.

Eximer theory has been extensively used to explain the spectra of the xanthene dye dimers (140-147). The predominant peak in the visible spectrum of the xanthene dye monomer is the $s_{0} \rightarrow s_{1}$ electronic transition occurring at approximately $600 \mathrm{~nm}$. This peak (including a higher energy shoulder) exactly resembles the shape of the porphyrin soret band. The dimer shows two peaks, one red, and one blue-shifted relative to the dye monomer (the shoulder disappears and is neglected in the theoretical analysis). The splitting of the monomer band upon formation of the xanthene dye dimer is used to calculate the angle made by the two faces of the dye dimer and the face-to-face dimer separation distance.

It is of interest to the author that the most cited paper on xanthene dye excimers (140) involves interpretation of data from an experiment where an anionic xanthene dye (eosin Y) was bound to specific cationic sites on a viologen polymer. The authors do not consider the possibility of association complexes with viologen (and omit the control experiment of addition of monomer viologen which would rule out association); instead, they consider only that the spectra are the result of xanthene dye excimers. However, the spectra exactly resemble the titration of $\mathrm{H}_{2} \mathrm{TCPP}^{4-}$ with $\mathrm{MV}^{2+}$. The author hopes to pursue the validity of interpretation of the xanthene dye experiments in future work by titrating eosin $Y$ with viologen. It may be that excimer theory has been misused in this case.

\section{The Effect of CMAB on the spectra and speciation of HTSPP $_{2}{ }^{4-}$.}

Another example of induced dimerization, shown in Figure 26 , demonstrates that organic cation-induced dimerization of anionic 
porphyrins is not unique to $\mathrm{PVS}^{0}$ and $\mathrm{H}_{2} \mathrm{TCPP}^{4-}$. Titration of $\mathrm{H}_{2} \mathrm{TSPP}^{4-}$ (or $\mathrm{H}_{2} \mathrm{TCPP}^{4-}$, with the cationic detergent, cetyltrimethylammonium bromide (CTAB), results in porphyrin dimerization at nearly equimolar detergent concentrations analogous to the induced dimerization of $1 \mu \mathrm{M} \mathrm{H}_{2} \mathrm{TCPP}^{4-}$ ( or $\mathrm{H}_{2} \mathrm{TSPP}^{4-}$ ) by $\mathrm{ca}$. equimolar concentrations of a cationic nitrogen macrocycle $\mathrm{N}_{8} \mathrm{H}_{8}^{8+}(69)$. At higher CTAB concentrations the dimer is dissociated (probably via solubilization into detergent micelles). Similarly, schmehl and Whitten (44) observed CTAB concentration dependent spectral changes (below the critical micelle concentration (CMC) but well above the porphyrin concentration) for TSPP ${ }^{4-}$ which resembled those obtained with viologens. The concentrations used were in the range where we observe an increasing absorbance and red-shifted Soret band.

Further examples from the literature show the effect is neither limited to porphyrins nor to the effect of cations on anionic dyes. The xanthene dye, eosin $Y$, showed spectral changes similar to Figure 26 when titrated with poly-I-lysine (144), as did acridine orange (a cationic dye) with poly (styrenesulfonic acid) (142).

The data shown in Figure 26 were collected by titration method 2 described in chapter III. $\mathrm{H}_{2} \mathrm{TSPP}^{4-}$ was used as its behavior has been found to be identical to $\mathrm{H}_{2} \mathrm{TCPP}^{4-}(69)$ and $\mathrm{H}_{2} \mathrm{TSPP}^{4-}$ dissolves readily in DI water allowing the inorganic salt concentration to be minimized.

The Formation of $\mathrm{H}_{2} \mathrm{TAPP}^{4+} / \mathrm{H}_{2} \mathrm{TCPP}^{4-}$ Heterodimers. The formation of porphyrin cationic/anionic heterodimers has been reported in the literature for both CuTMPyP $\mathrm{P}^{4+} / \mathrm{H}_{2} \mathrm{TCPP}^{4-}$ in 758 sucrose $\mathrm{w} / \mathrm{w}$ in water (67) and for $\mathrm{H}_{2} \mathrm{TMPyP}^{4+} / \mathrm{H}_{2} \mathrm{TSPP}^{4-}(64)$ in $50 \%$ acetone:water. In Figure 27 we show the spectra in DI water of $\mathrm{H}_{2} \mathrm{TCPP}^{4-}$ and $\mathrm{H}_{2} \mathrm{TAPP}^{4+}$ (aggregated, actual formal charge unknown) and of the heterodimer formed from equimolar concentrations of each. The experiments described in the literature used mixed solvents, thus the anionic tetraphenylporphyrin monomer spectra were red-shifted relative to the monomer spectra shown in Figure 


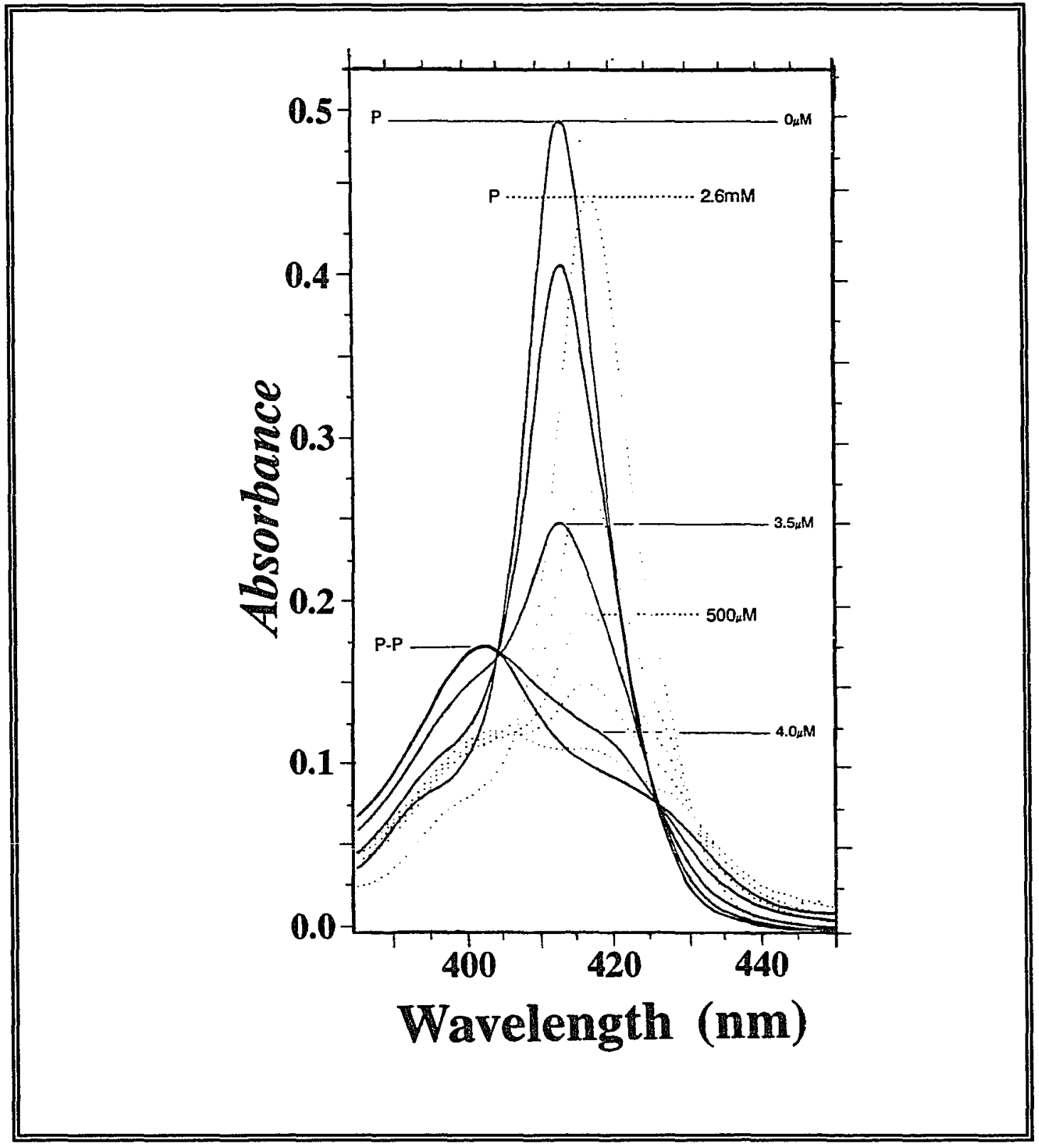

Figure 26. Titration of $1 \mu \mathrm{M} \mathrm{B}_{2}$ TSPP ${ }^{4-}$ with CTAB. Selected spectra from the titration are shown ( 45 points were taken). Selected concentrations of titrant are indicated on the graph. "...." indicates high [titrant]. The $\mathrm{pE} \approx 6$, the solution was not buffered. $\mathrm{P}=$ porphyrin $\mathrm{P}-\mathrm{P}=$ porphyrin dimer.

27. This resulted in a greater reported blue shift upon formation of the heterodimer relative to our data. In this experiment the heterodimer spectrum resembles the sum of the cationic and anionic dimer. 
The heterodimers were not dissociated either by high temperature $\left(50^{\circ} \mathrm{C}\right)$ or by the addition of $\mathrm{MV}^{2+}$ up to $40-100 \mathrm{mM}$. Viologen was added to investigate the possibility that the observed spectrum was the result not of heterodimer formation, but of $\mathrm{H}_{2} \mathrm{TCPP}^{4-}$ and $\mathrm{H}_{2} \mathrm{TAPP}^{4+}$ dimers. Since we have observed that the addition of $\mathrm{MV}^{2+}$ induces dissociation of $\mathrm{H}_{2}$ TCPP $^{4-}$ dimers (shown in a later section), and since the addition of viologen caused no change in the observed spectrum, we conclude that the observed spectrum is the result of heterodimer formation.

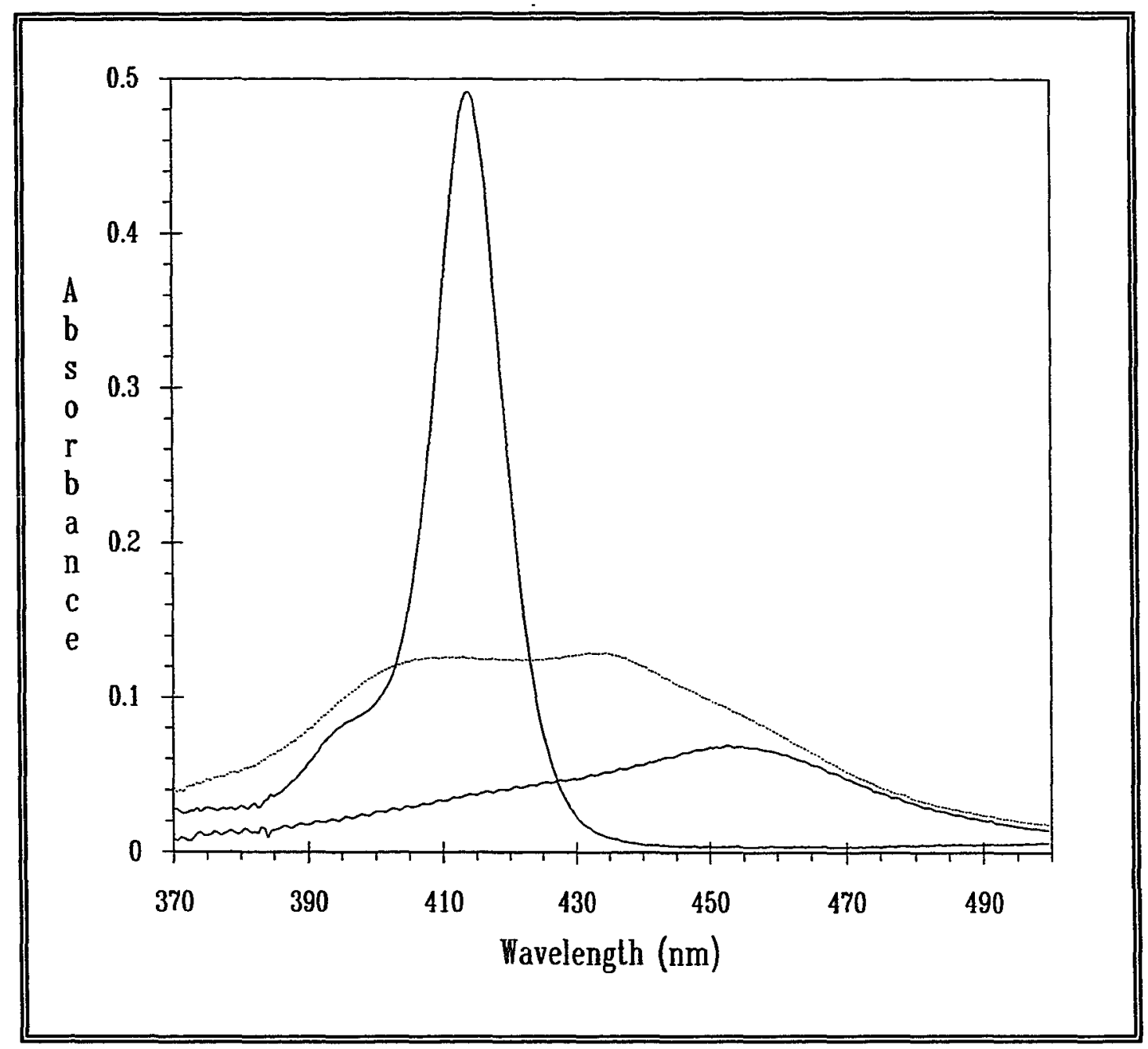

Figure 27. Spectrum of $\mathrm{H}_{2} \mathrm{TCPP}^{4-} /$ heterodimers. Spectrum of the $\mathrm{H}_{2}$ TCPP $^{4-}$ monomer and aggregated $\mathrm{H}_{2}$ TAPP $^{4+} ; \lambda_{\text {ax }} \approx 414$ and $455 \mathrm{~nm}$, respectively. Spectrum of the heterodimer is shown as "..." . The concentration of each porphyrin $=1 \mu \mathrm{M}$. 
H$_{2}$ TSPP4- Dimer Formation by $3 \mathrm{M}$ Nacl: Dissociation Induced by Crown Ether. Porphyrin dimerization in response to inorganic cations has long been reported in the literature (83-85); both the salt concentration needed to observe dimerization and the resultant dimer spectra differ from my observations. The weakened (but not shifted) Soret band upon addition of inorganic cations was attributed to dimerization.

Our observations, along with those of Firman et al. (69), identify the spectrum of the anionic tetraphenylporphyrin dimer (induced by organic salts) as severely weakened (80\% loss) and blue-shifted by approximately 7-14 $\mathrm{nm}$. The $\mathrm{H}_{2} \mathrm{TSPP}^{4-}$ dimer spectrum induced by $3 \mathrm{M} \mathrm{NaCl}$ is blue-shifted by $6 \mathrm{~nm}$ and weakened by $80 \%$ relative to the monomer spectrum. The dimer spectrum induced by inorganic cations was broadened and less blue-shifted than the dimer spectrum observed with organic cations. The subject of spectral line shape change in response to alkali metal cations is investigated in the last section of this chapter.

Porphyrins are often assumed to behave as normal ions. To be explicit, anionic tetraphenylporphyrin/cation interactions should be limited to electrostatic attraction. This restricts the salt interaction to the carboxylate site. Cation-induced anionic tetraphenylporphyrin dimerization $\left(\mathrm{H}_{2} \mathrm{TCPP}^{4-}, \mathrm{H}_{2} \mathrm{TSPP}^{4-}\right)$ is thus attributed to reduced charge repulsion between $\mathrm{H}_{2} \mathrm{TCPP}^{4-}$ molecules via coulombic shielding of the charged carboxylate groups. This theory does not consider the hydrophobic nature of the porphine core.

In Figure 28 we show $\mathrm{H}_{2} \mathrm{TSPP}^{4-}$ dimers induced by $3 \mathrm{M} \mathrm{NaCl}$. These dimers are dissociated by $0.1 \mathrm{mM}$ concentrations of the crown ether 15crown-5. Assuming that each crown ether chelates a single sodium ion, the concentration of the chelator is too low to explain the dimer dissociation by the model that the sodium is removed from the sodium sulfonate "ion pairs" of the tetraphenylporphyrin. 
The experimental data were collected by titration method $2 \mathrm{~B}$ described in chapter III. The sodium salt of $\mathrm{H}_{2} \mathrm{TSPP}^{4-}$ was chosen for

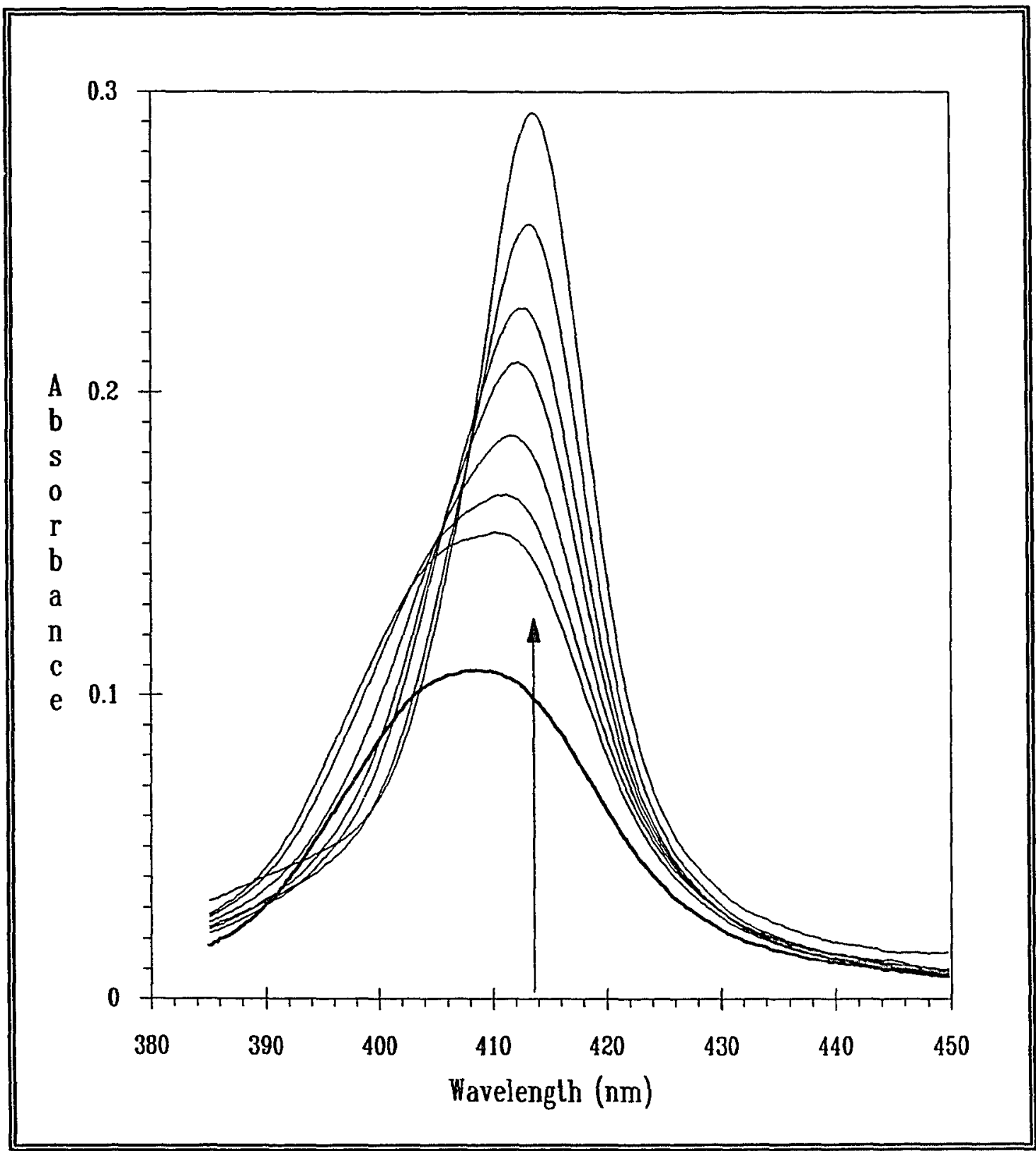

Figure 28. Dissociation of dimerized H ISPP $^{4-}$ by the addition of 15-crown-5 ether. The porphyrin dimerization was induced by $3 \mathrm{M} \mathrm{NaCl}$ (dark line) at $\mathrm{pH} \approx 6$. The $\mathrm{pH}$ was adjusted to $\approx 9$ with NaOH; no spectral change was observed. The arrow $T$ indicates crown ether induced spectral changes, concentrations (mM) of crown ether are: $1.7,8.4,16.7$, $33.4,50.0,66.3,100 ;\left[\mathrm{H}_{2} \mathrm{TSPP}^{4-}\right]=1 \mu \mathrm{M}, \mathrm{T}=20^{\circ} \mathrm{C}, \mathrm{pH} \approx 9$. 
this experiment since this porphyrin dissolves in DI water, thus the sodium concentration was known (as compared with $\mathrm{H}_{2} \mathrm{TCPP}^{4-}$ where the porphyrin must be dissolved in base and buffered in order to avoid precipitation).

MMR Evidence for Association of HeTCPP ${ }^{4-}$ with MV ${ }^{2+}$ : Dissociation of H$_{2}$ TCPP ${ }^{4-}$ Dimers with MV $^{2+}$

With the help of Dr. David Peyton we were able to confirm the presence of $\mathrm{H}_{2} \mathrm{TCPP}^{4-} / \mathrm{MV}^{2+}$ association complexes via $300 \mathrm{MHz}{ }^{1} \mathrm{H} \mathrm{NMR}$ in buffered aqueous solution. This evidence allowed us to correlate the red-shifted and weakened UV/VIS absorbance spectrum with porphyrin/viologen association complexes, thus was critical to the interpretation of the data used in determination of the equilibrium constants. Moreover, we learned that the addition of $\mathrm{MV}^{2+}$ dissociated porphyrin dimers (although this evidence is less strong), which was important due to the theory favored in the literature that an increase in ionic strength leads to induced porphyrin dimerization.

The preparation of the solutions used in this experiment are explicitly described in chapter III and in Table VII below. Due to the limits of the sensitivity of NMR, the porphyrin concentration had to be in the range where we expect dimerization (or aggregation). This is confirmed by contrasting the NMR spectrum of $\mathrm{H}_{2} \mathrm{TCPP}^{4-}$ in organic solvents (spectrum not shown) with the relatively broadened peaks of the spectra in buffered aqueous solvent as shown in the top NMR spectrum in Figure 29 and in Chapter II, Figure 13.

The experiment was designed to observe shifts in the viologen NMR peaks as a function of $\mathrm{H}_{2} \mathrm{TCPP}^{4-}$ concentration (Figure 29). Therefore the viologen concentration was kept constant and the porphyrin concentration varied. We used equilibrium constants calculated from experiments described in Chapter $\mathrm{V}$ to estimate the porphyrin:viologen concentration ratios needed to give the percent of complexed viologen 
TABIE VII

SOLUTIONS USED IN THE $300 \mathrm{MHZ}$ ' $\mathrm{H}$ MMR STUDY OF $\mathrm{H}_{2} \mathrm{TCPP}^{4-} / \mathrm{MV}^{2+}$ COMPLEXATION

\begin{tabular}{|c|c|c|}
\hline $\begin{array}{c}\text { 8 of } \mathrm{Mv}^{2+} \text { Complexed to } \\
\mathrm{H}_{2} \mathrm{TCPP}^{\mathrm{s}}-\end{array}$ & {$\left[\mathrm{Mv}^{2+}\right]$} & {$\left[\mathrm{H}_{2}^{\mathrm{TCPP}^{4-}}\right]$} \\
\hline 0 & $0.50 \mathrm{mM}$ & $0.0 \mathrm{mM}$ \\
\hline 7.4 & $0.50 \mathrm{mM}$ & $0.050 \mathrm{mM}$ \\
\hline 17.8 & $0.50 \mathrm{mM}$ & $0.125 \mathrm{mM}$ \\
\hline 25.7 & $0.50 \mathrm{mM}$ & $0.188 \mathrm{mM}$ \\
\hline 0 & $0.0 \mathrm{mM}$ & $0.625 \mathrm{mM}$ \\
\hline
\end{tabular}

given in Table VII. The calculated solution concentration of each species is given in table form on the lower half of the figure.

The bottom spectrum in the Figure is of viologen only, the next

three higher spectra show the addition of progressively higher concentrations of porphyrin. Focusing on the porphyrin peaks, which are shaded, notice that they are sharper than those in the spectrum where viologen is absent (top). In the absence of $\mathrm{MV}^{2+}$ a broad peak was observed for the porphyrin phenyl ortho and pyrrole $B$ protons due to the extensive aggregation which is expected at this high porphyrin concentration. However, the phenyl ortho doublet was sharpened in the presence of $\mathrm{MV}^{2+}$ (the pyrrole $B$ protons are further broadened and partially obscured by the viologen) as the porphyrin concentration increased which we interpret as evidence for viologen induced porphyrin dimer dissociation.

Studies with molecular models show that two viologens can easily be accommodated across the face of a single porphyrin molecule; this interaction is illustrated in Figure 30, top.

If the porphyrin/viologen association is driven primarily by coulombic attraction, we would expect that the viologen would be 


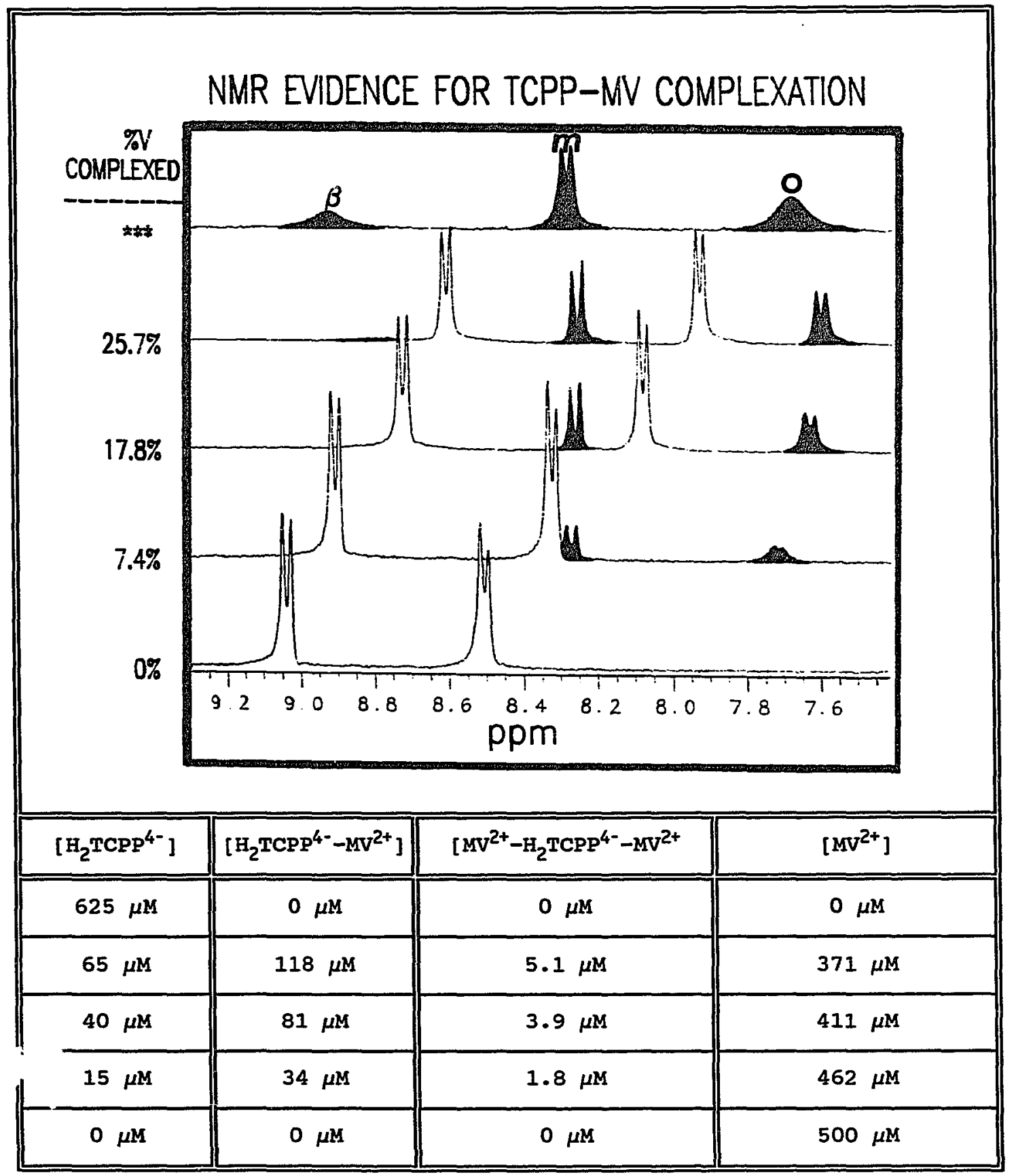

Figure 29. $300 \mathrm{MHz}{ }^{1_{H}}$ NMR study of $\mathrm{B}_{2} \mathrm{TCPP}^{4-} / \mathrm{MV}^{2+}$ association. $\left[\mathrm{MV}^{2+}\right]=$ constant; $q$ of viologen complexed is shown at the left. From bottom to top: Mv ${ }^{2+}$ only, next 3 spectra are of increasing [H,TCPP ${ }^{4-}$ ] at constant [MV ${ }^{2+}$ ], top spectra is of H TCPP $^{4-}$ only. The actual total concentrations are given in Table VII. The calculated solution concentration of each species is given in table form below the graph. 
oriented with its positive charges in close proximity to the negative carboxylate groups. The viologen ring currents would then most affect the chemical shifts of the meta protons. What is actually observed, is that the meta protons are the least affected (Figure 30, bottom). The changes in the chemical shifts are consistent with the viologen oriented over the inner edge of the face of the porphine ring. This is shown by the upfield shift of the resonance absorbance of the viologen protons along with the porphyrin's ß-pyrrole and ortho protons. These observations are analogous to a 400-MHz $\mathrm{H}^{1}$ NMR study of the complexation of $\mathrm{H}_{2}$ TMPyP $^{4+}$ dimer with 9,10-anthraquinone-2-sulfonate ${ }^{1-}$ (AQßS ${ }^{1-}$ ) in $\mathrm{D}_{2} \mathrm{O}$ which also showed concentration dependent resonance shifts for both species (58). As in the complexation of $\mathrm{H}_{2} \mathrm{TCPP}^{4-}$ by $\mathrm{MV}^{2+}$, the shifts were consistent with a face-to-face porphyrin/quencher complex. Also analogous to the data presented here, is that the stoichiometry for the porphyrin/quencher complex was greater than 1:1 (however, the authors were unable to determine the stoichiomstry of the higher complex). The magnitude of the changes in the $M V^{2+}$ chemical shifts correlated well with the estimated concentration of complexed $\mathrm{MV}^{2+}$ (calculated from equilibrium constants whose determination is described in Chapter V) as is evidenced by the linear fit of the chemical shifts when plotted versus the percent of complexed viologen (Figure 30 , bottom) .

\section{SUMRARY}

From this collection of examples it appears that coulombic shielding by ions cannot, by itself, explain salt induced porphyrin dimerization. of the two viologens $\mathrm{MV}^{2+}$ is a better candidate to induce porphyrin dimerization than $\mathrm{PVS}^{0}$ by the coulombic model. The addition of $\mathrm{MV}^{2+}$ increases the ionic strength (predicting induced dimerization via coulombic shielding) in contrast to zwitterionic Pvs $^{0}$ whose addition represents the increasing concentration of a strong dipole. The major 


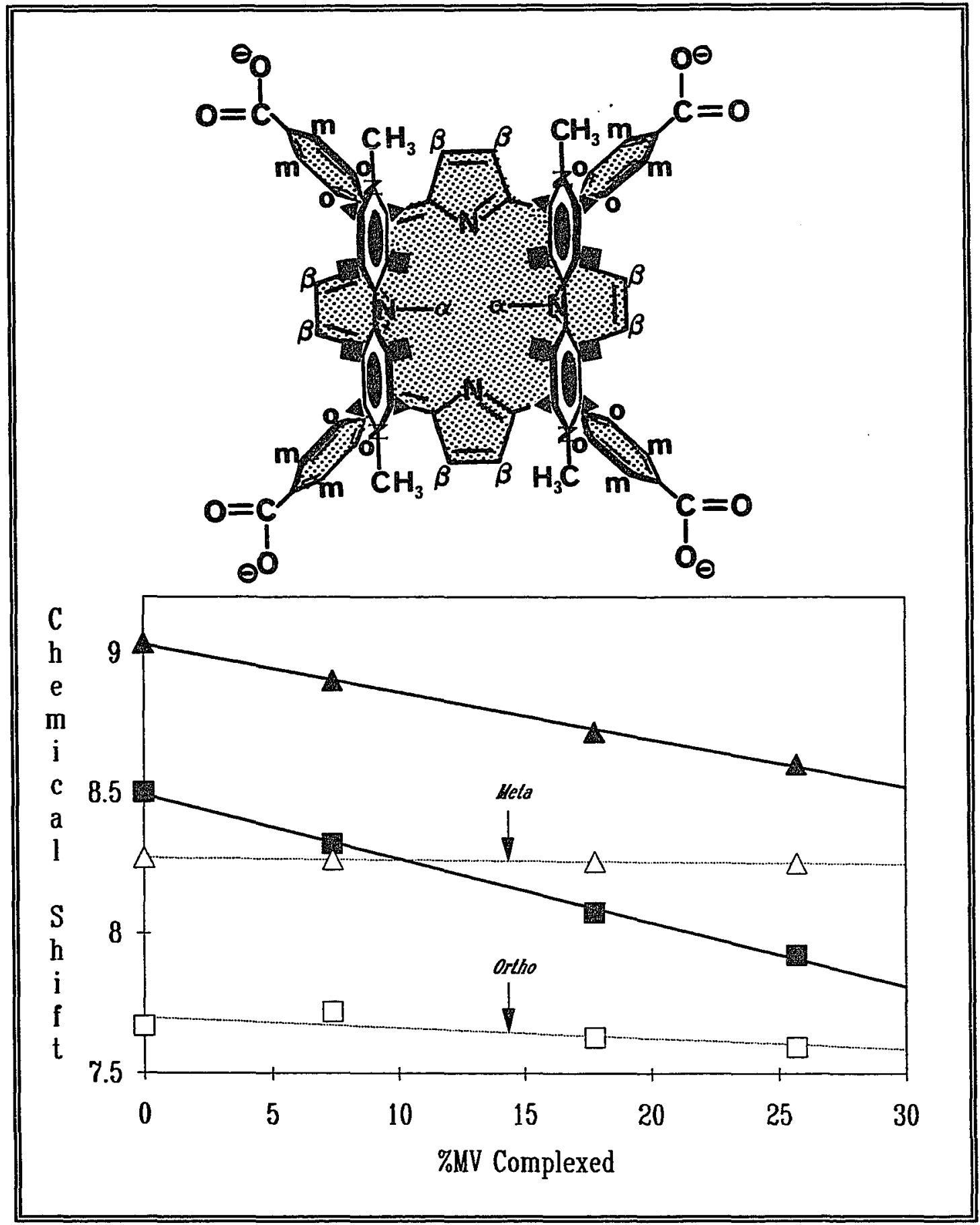

Eigure 30. Correlation of the 8 of $\mathrm{H}_{\mathrm{TCPP}} \mathrm{TC}^{4-}$-complexed $\mathrm{MV}^{2+}$ with the change in the chemical shifts. Top; illustration of the orientation of $\mathrm{MV}^{2+}$ molecules over H,TCPP ${ }^{4-}$ consistent with the MMR data. Bottom; plot of the chemical shifts versus the calculated concentration of porphyrin-complexed viologen. $\Delta$ and on $\mathrm{Mv}^{2+}$ (top) correspond to graph markers. 
driving force for the dimerization appears to be hydrophobic, i.e.solvent exclusion.

The "hydrophobic effect" has long been invoked by biochemists to explain the phenomenon of self-assembly of biological structures such as DNA, membranes and proteins. This concept describes the tendency of nonpolar solutes to adhere to each other in aqueous solution. Recently, Ben-Naim (148) has suggested that this effect might better be explained as solvent-induced intramolecular hydrophilic interaction, a relevant example being solvent-induced intramolecular hydrogen bonding. This concept is attractive as it is easily translated to visual images of solute-solute interactions. In the specific case of $\mathrm{H}_{2} \mathrm{TCPP}^{4-}$ dimerization, the four carboxylate groups of one porphyrin molecule could hydrogen bond through a water bridge with the corresponding carboxylate groups of a second porphyrin. This theory might help to explain the unusually low solubility of $\mathrm{H}_{2} \mathrm{TCPP}^{4-}$. It is more difficult to rationalize the dimerization induced by large organic molecules such as detergents and organic salts such as the nitrogen macrocycle $\mathrm{N}_{8} \mathrm{H}_{8}{ }^{8+}$; it may be that the solubility of the porphyrin is lowered by the presence of additional organic molecules with low solubility.

The author has a working hypothesis to explain the novel induced porphyrin dimerization observed with $\mathrm{PVS}^{0}$ but not with $\mathrm{MV}^{2+}$. The model involves two observations. The first contrasts the structure of the two viologens and considers their accommodation across the face of the porphyrin macrocycle (Figures 30 and 31 ). The drawings shown in Figure 31 below attempt to illustrate the possible spacial orientation of the propylsulfonate groups in the $\mathrm{H}_{2} \mathrm{TCPP}^{4-} / \mathrm{PVS}^{0}$ complex. In the upper drawing the dark line represents the plane of the porphyrin macrocycle, and indicates that the porphyrin is viewed from the edge. In order for the aromatic rings of the porphyrin and viologen to interact, the . propylsulfonate groups must be twisted up out of the porphyrin plane where the negatively charged sulfonate groups repel one another. If the 
groups are twisted outward and away from a second viologen molecule, as shown in the lower drawing, then they might be repelled by the carboxylate groups on the porphyrin. In the lower arawing, the crosshatched region shows the projection of the porphyrin onto the plane of the paper with the two viologens oriented above the plane. By

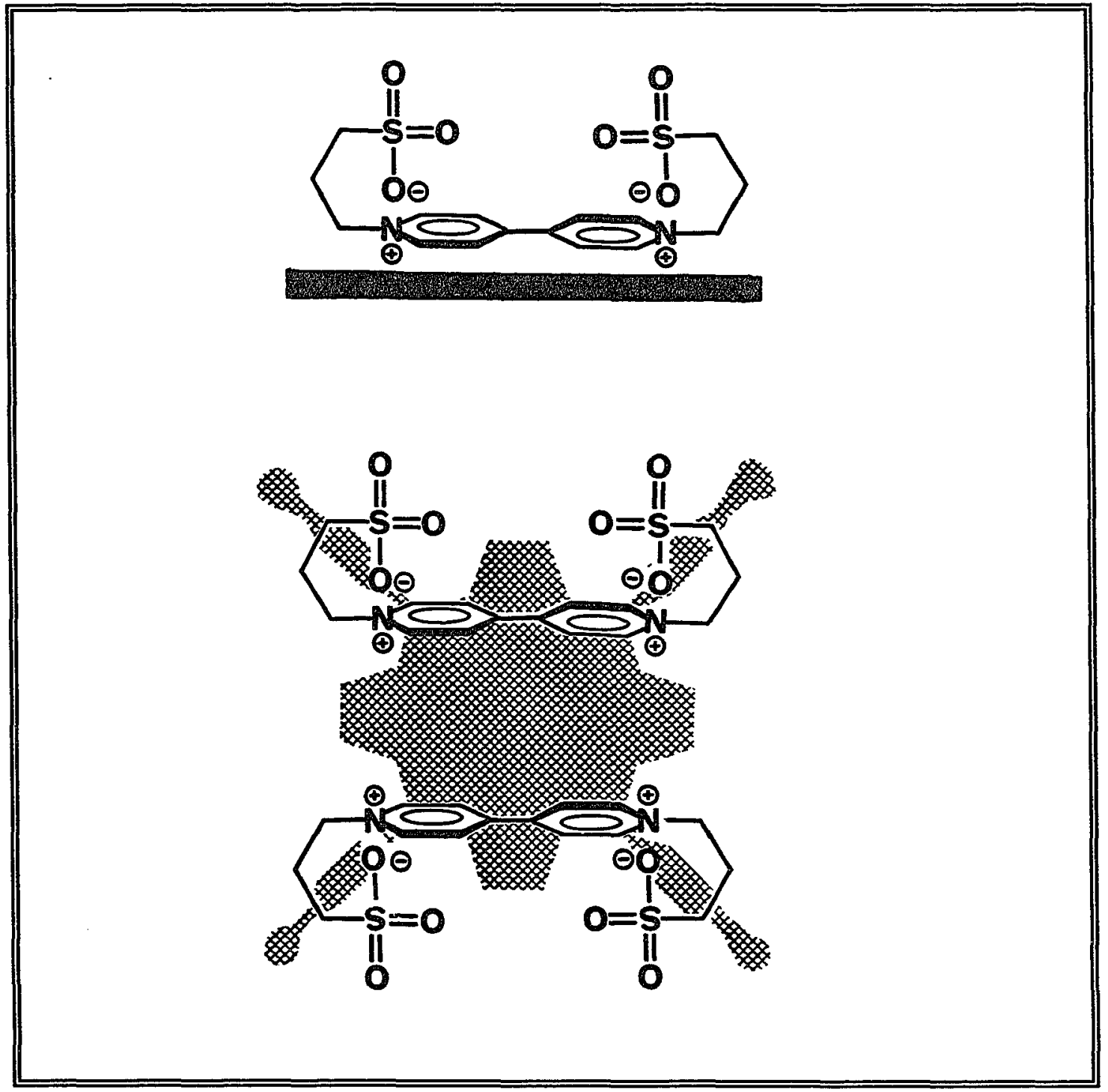

Figure 31. Illustration of two possible orientations for the propy isulfonate group in the $\mathrm{H}_{2}$ TCPP $^{4-} /$ PVS $^{0}$ association complex.

comparing Figures 30 and 31 one can see that PVS ${ }^{0}$ is less easily 
accommodated by one face of the porphyrin than $\mathrm{MV}^{2+}$.

The second piece of information used to develop this model is that porphyrins appear to form face-to-face associations with only one face $(61,68)$. Thus, the addition of pyridine to face-to-face linked porphyrins dimers causes the faces to move apart (or dimers to dissociate). This is the model the author favors to explain the $\mathrm{MV}^{2+}$ induced $\mathrm{H}_{2} \mathrm{TCPP}^{4-}$ dimer dissociation and why $\mathrm{I}$ have shown the viologens complexed to a single face of the porphyrin.

In Figure 23B, porphyrin dimerization is induced in the region of the titration where the $1: 1$ complex is in equilibrium with the $1: 2$ $\left(\mathrm{H}_{2} \mathrm{TCPP}^{4-}: \mathrm{PVS}^{0}\right)$ complex. It may be more favorable for the porphyrin to associate with another porphyrin, thereby releasing the very soluble viologens, than to from a $1: 2$ complex.

Similarly, the concentration-dependent effect of the detergents and the nitrogen macrocycle on porphyrin speciation in aqueous solution cannot be explained without considering how the addition of the organic salt affects the porphyrin solubility. Induced dimerization of organic dyes might be explained by separating the free energy changes (from equilibrium constants) into $\Delta \mathrm{H}$ and $\Delta \mathrm{s}$ terms by NMR and/or absorbance temperature studies.

Although new questions arise from these data, our objectives of correlating the weakened and blue-shifted porphyrin spectrum with porphyrin dimers and the red-shifted and weakened porphyrin spectrum with complexation (by viologen) was achieved. In addition, we are now better able to predict conditions where dimer formation may be induced.

THE EFFECT OF INORGANIC CATION CONCENTRATION ON THE BEER'S LAW PLOTS

Pasternack and coworkers (83-86) found that salts decreased the linear region of the Beer's law plots, which was interpreted as saltinduced $\mathrm{H}_{2} \mathrm{TCPP}^{4-}$ dimerization. However, it was necessary to perform the $\mathrm{H}_{2} \mathrm{TCPP}^{0} / \mathrm{viologen}$ complexation studies in buffered solution to eliminate 
error due to $\mathrm{pH}$ induced spectral changes. Therefore conditions where only monomer (free base) exists in buffered solution had to be carefully defined. This was accomplished in the normal way by performing a series of Beer's law experiments under different conditions of temperature, pH, and sait concentration. From these experiments we found that $\mathrm{H}_{2} \mathrm{TCPP}^{4-}$ is in monomer form in buffered solution (5 mM KH $2 \mathrm{PO}_{4} / \mathrm{NaKHPO}_{4}$ ) only at surprisingly low porphyrin concentrations, below $2 \mu \mathrm{M}$. We judged the monomer concentration range to be defined as the linear region of the Beer's law plots.

Beer's law simply states that the absorbance is proportional to the product of the concentration $(M)$ of the absorbing species and the distance the light beam travels through the solution being measured (the pathlength in $\mathrm{cm}$ ). Thus a proportionality constant can be determined, termed the "absorptivity" in units of $\mathrm{M}^{-1} \mathrm{~cm}^{-1}$. The Beer's law experiment holds all parameters constant except the concentration of the absorbing species. What is expected is that at low concentrations the data can be described as a linear function, where the slope of the line is the proportionality constant, the absorptivity. At high concentration Beer's law no longer holds due to some interaction between the absorbing species. The concentration at which this effect becomes significant is usually expected to be in the 10 to $100 \mathrm{mM}$ range. Thus $\mathrm{H}_{2}$ TCPP $^{4-}$ is very unusual since deviation from Beer's law occurs 4 to 5 orders of magnitude earlier than expected.

However, the premise that deviation from linearity of the Beer's law plot corresponds to the formation of dimers (or aggregates) in solution may not be correct and in future experiments we would like to test this assumption by the method presented in the last section of this chapter. Dimerization is not the only phenomenon which can give rise to loss of linearity in the Beer's law plots, and it appears that we have data which bring the assumption into question.

Presented in Table VIII and IX are the experimental conditions and 
a summary of the results of the Beer's law experiments contained within this section, respectively. The experiment number corresponds to the numbers within the figure legends on the following pages. The method

TABLE VIII

BEER'S LAW EXPERIMGNTS: SUMMARY OF EXPERIMIANTAI CONDITIONS

\begin{tabular}{|c|c|c|c|c|c|c|c|c|c|}
\hline \multirow{2}{*}{$\underset{\#}{\text { Expmt }}$} & \multirow{2}{*}{$\begin{array}{l}\text { Method } \\
\text { (Chapt } \\
\text { III) }\end{array}$} & \multirow{2}{*}{$\begin{array}{l}\text { Porphyrin } \\
\text { Studied }\end{array}$} & \multirow{2}{*}{$\begin{array}{l}\text { Porphyrin } \\
\text { Concentration } \\
\text { Range Studied }\end{array}$} & \multirow{2}{*}{$\begin{array}{c}\lambda_{\max }{ }^{*} \\
\lambda_{i} \rightarrow \lambda_{f}\end{array}$} & \multicolumn{5}{|c|}{ Experimental Conditions } \\
\hline & & & & & pH & Burfer & Temp. & tiont & {$[+i o n] \star$} \\
\hline $1 A$ & $2 A$ & $\mathrm{H}_{2} \mathrm{TSPP}^{4-}$ & $0.08 \rightarrow 1.9 \mu \mathrm{M}$ & 414,435 & $\approx 5$ & None & $20^{\circ} \mathrm{C}$ & $\mathrm{H}^{+}$ & $\approx .01 \mathrm{~mm}$ \\
\hline 1B & 2B & $\mathrm{H}_{2} \mathrm{TSPP}^{4-}$ & $1.9 \mu \mathrm{M}$ & 414,435 & $\approx 5$ & None & $20^{\circ} \mathrm{C}$ & $\mathrm{Na}^{+}$ & $\approx 2 \mathrm{mM}$ \\
\hline $1 \mathrm{C}$ & 28 & $\mathrm{H}_{2} \mathrm{TSPP}^{4-}$ & $1.9 \mu \mathrm{M}$ & $435-414$ & $\approx 8$ & None & $20^{\circ} \mathrm{C}$ & $\mathrm{Na}^{+}$ & $\approx 2 \mathrm{~mm}$ \\
\hline 2 & 1 & $\mathrm{H}_{2} \mathrm{TCPP}^{4-}$ & $0.01+60 \mu \mathrm{M}$ & $414.6 \rightarrow 414.5$ & $\approx 10$ & None & $35^{\circ} \mathrm{C}$ & $\mathrm{Na}^{+}$ & $\approx 0.1 \mathrm{mH}$ \\
\hline 3 & 1 & $\mathrm{H}_{2} \mathrm{TCPP}^{4-}$ & $0.01+40 \mu \mathrm{M}$ & $414.3 \rightarrow 414.3$ & $=10$ & None & $20^{\circ} \mathrm{C}$ & $\mathrm{Na}^{+}$ & $\approx 0.1 \mathrm{mot}$ \\
\hline \multirow[t]{2}{*}{4} & \multirow[t]{2}{*}{1} & \multirow[t]{2}{*}{$\mathrm{H}_{2} \mathrm{TCPP}^{4-}$} & \multirow[t]{2}{*}{$0.08 \rightarrow 80 \mu \mathrm{M}$} & \multirow[t]{2}{*}{$414+414$} & \multirow[t]{2}{*}{7.0} & \multirow[t]{2}{*}{$5.0 \mathrm{~mm}$ Phos. } & \multirow[t]{2}{*}{$20^{\circ} \mathrm{C}$} & $\mathrm{Na}^{+}$ & $\approx 2.5 \mathrm{~m}$ \\
\hline & & & & & & & & $k^{+}$ & $5.0 \mathrm{mH}$ \\
\hline \multirow[t]{2}{*}{5} & \multirow[t]{2}{*}{$2 A$} & \multirow[t]{2}{*}{$\mathrm{H}_{2} \mathrm{TCPP}^{4-}$} & \multirow[t]{2}{*}{$0.004 \rightarrow 1.8 \mu \mathrm{M}$} & \multirow[t]{2}{*}{$414.2 \rightarrow 414.2$} & \multirow[t]{2}{*}{7.0} & \multirow[t]{2}{*}{$5.0 \mathrm{~mm}$ Phos. } & \multirow[t]{2}{*}{$21^{\circ} \mathrm{C}$} & $\mathrm{Na}^{+}$ & $\approx .25 \mathrm{~mm}$ \\
\hline & & & & & & & & $\mathrm{K}^{+}$ & $5.0 \mathrm{mM}$ \\
\hline \multirow[t]{2}{*}{6} & \multirow[t]{2}{*}{1} & \multirow[t]{2}{*}{$\mathrm{H}_{2} \mathrm{TCPP}^{4-}$} & \multirow[t]{2}{*}{$0.06 \rightarrow 80 \mu \mathrm{M}$} & \multirow[t]{2}{*}{$414 \rightarrow 410$} & \multirow[t]{2}{*}{7.0} & \multirow[t]{2}{*}{$5.0 \mathrm{~mm}$ Phos. } & \multirow[t]{2}{*}{$20^{\circ} \mathrm{C}$} & $\mathrm{Na}^{+}$ & $503 \mathrm{~mm}$ \\
\hline & & & & & & & & $k^{+}$ & $5.0 \mathrm{mM}$ \\
\hline
\end{tabular}

* where i=initial and $f=f$ inal, wavelength in $\mathrm{mm}_{;}$Phos. $=\mathrm{KH}_{2} \mathrm{PO}_{4} / \mathrm{NaKHPO}_{4} ; *+i o n=$ alkali metal cation.

refers to the descriptions in Chapter III, where " 1 " corresponds to individually prepared solutions whose absorbance is read in cells of varying pathlength and normalized to a $1 \mathrm{~cm}$ pathlength cell.

Method "2A" corresponds to the titration method also described in Chapter III where the absorbance data is measured in a 1 or $2.2 \mathrm{~cm}$ pathlength cell and normalized to $1 \mathrm{~cm}$. The linear regression method was also described in Chapter III. The Beer's law experiments are 
presented in the order of the most to least favorable conditions for extension of the linear range to "high" porphyrin concentrations. The results are consistent with the accepted literature model: high temperature, high $\mathrm{pH}$, and minimal cation concentrations extend the linear region of the Beer's law plot. With the exception of experiments 1A-C, the data are presented without further explanation.

The reader will notice that there is an inconsistency between the precision and the accuracy of the absorptivity determinations shown in Table IX. This is real and its source is explained by the unexpected results in the following section where we show that the absorptivity of $\mathrm{H}_{2} \mathrm{TCPP}^{4-}$ is, surprisingly, a function of the alkali metal cation concentration.

TABLE IX

BEER'S IAW EXPERIMENTAL RESULTS

\begin{tabular}{|c|c|c|c|c|c|c|}
\hline $\begin{array}{c}\text { Experiment } \\
\#\end{array}$ & Absorptivity* & $\begin{array}{c}\text { Standard } \\
\text { Deviation } \\
\text { (Absorptivity) }\end{array}$ & $r^{2}$ & $\begin{array}{c}\text { Degrees } \\
\text { of } \\
\text { Freedom }\end{array}$ & $\begin{array}{c}\text { Total Mumber of } \\
\text { Data Points }\end{array}$ & $\begin{array}{c}\text { Maximum H2TCPP4- } \\
\text { Concentration in } \\
\text { Linear Region }\end{array}$ \\
\hline 2 & 485,000 & 1,340 & 0.9998 & 27 & 30 & $40.0 \mu \mathrm{M}$ \\
\hline 3 & 508,000 & 1,520 & 0.9998 & 24 & 31 & $8.0 \mu \mathrm{M}$ \\
\hline 4 & 484,000 & 2,380 & 0.9997 & 12 & 23 & $2.0 \mu \mathrm{M}$ \\
\hline 5 & 472,000 & 660 & 0.99997 & 14 & 29 & $0.7 \mu \mathrm{M}$ \\
\hline 6 & 404,000 & 15,100 & 0.995 & 4 & 23 & $\approx 0.3 \mu \mathrm{MM}$ \\
\hline
\end{tabular}

* in units of $\mathrm{M}^{-1} \mathrm{~cm}^{-1}$ and corrected for the density of water at the reported temperature. 2) There may not be a linear region.

\section{Experiment \#1: The Effect of Minimal Alkali Metal Ion}

Concentration. This experiment studies the effect of minimal ionic strength and pH on the porphyrin spectrum. The spectral series beginning with the spectrum with the lowest absorbance (at $414 \mathrm{~nm}$ ) and ending with the spectrum indicated by a dotted line was described as experiment 1A in Table VIII. This is a "Beer's law" type of experiment, 
where each curve in the figure illustrates the effect of an incremental increase in the porphyrin concentration. All other parameters are held constant as described in Table V, column A (designated as method 2A). The spectral series indicated by dark lines and showing higher absorbance at $414 \mathrm{~nm}$ than the spectrum designated by a dotted line is defined as experiment $1 \mathrm{C}$ in Table VIII. This experiment studied the effect of NaOH addition on the porphyrin spectrum at constant porphyrin concentration. The methodology was described by Table V, column B (method 2B).

Experiment 1B (Table VIII) is not shown. The incremental increase of $\mathrm{NaCl}$ at constant porphyrin concentration induced no change in the porphyrin spectrum $\left(\left[\mathrm{H}_{2} \mathrm{TSPP}^{4-}\right]=1.9 \mu \mathrm{M}\right.$, dotted line in Figure 32$)$.

Alkali metal salts were eliminated from the anionic sites on the glassware by exchanging with protons as follows: any glassware to come in contact with the porphyrin was rinsed with ultrapure $\mathrm{HCl}$ and exchanged 5 times over a period of 30 hours with DI water to remove any remaining acid followed by drying in an oven. The porphyrin solution (titrant 1) was weighed, dissolved in DI water and loaded into the buret as described in the methods section (Chapter III). The salt (titrant 2) and the base (titrant 3 ) were prepared as a dilution from concentrated solutions as described previously. Saturated (50\%, to minimize contamination from carbonate) ultrapure NaOH was diluted to a final concentration of approximately $10 \mathrm{mM}$ immediately before use.

The spectral changes observed in this experiment were interpreted in Chapter II. Briefly, relative absorptivities of the two bands in the Soret region indicate the equilibrium concentration ratio of the free base (414 $\mathrm{nm}$ band) and monocation species (435 nm band). Each incremental increase in the porphyrin concentration represents the addition of a weak base which raises the pH (slightly). This results in a shift in the equilibrium towards the free base. This subtle acid/base chemistry is observed as a relative gain in $414 \mathrm{~nm}$ band (free base) and 


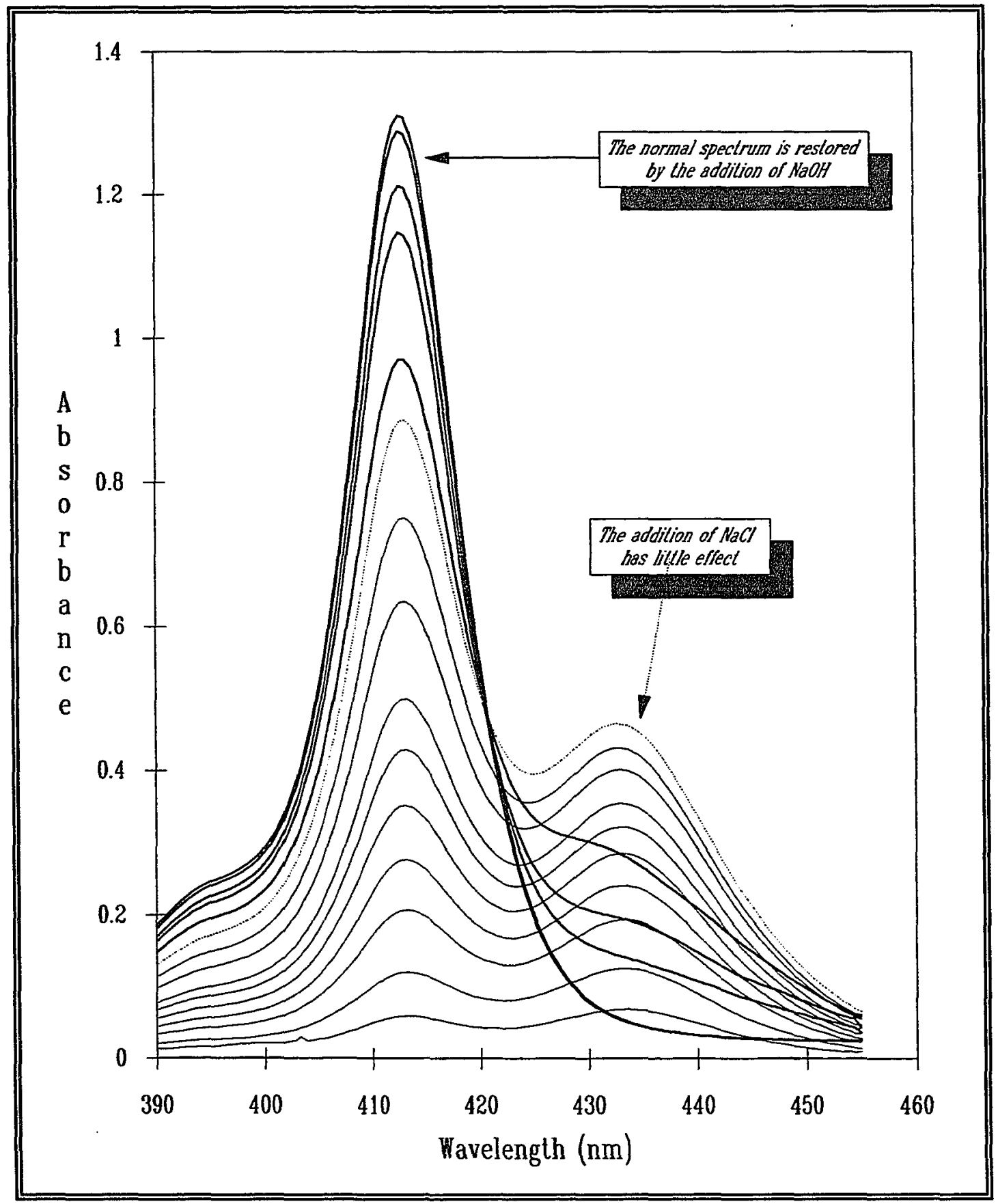

Figure 32. The effect of minimal ionic strength and low pE on the Beer's law spectra of $\mathrm{H}_{2} \mathrm{TSPP}^{4-}$. The $\left[\mathrm{H}_{2} \mathrm{TSPP}^{4-}\right.$ ] corresponding to spectra of increasing absorbance are, respectively: $0.08,0.2,0.4,0.6,0.76,0.9,1.3,1.5$ and $1.7 \mu \mathrm{M}$. ".." indicates $1.9 \mu \mathrm{M}$ F.TSPP ${ }^{4-}$, where $\mathrm{NaCl}$ addition had little effect (result not shown). The solid heavy lines indicate the titration of $1.9 \mu \mathrm{M} \mathrm{H}$ TSPP ${ }^{4-}$ with NaOH of approximate concentrations $(\mu \mathrm{M}): 1,20,30$ and 50 . 
a relative loss in the $435 \mathrm{~nm}$ band (monocation). When the porphyrin concentration reached $1.9 \mu \mathrm{M}$ (represented by a dotted line in the figure) the titrant was changed to $\mathrm{NaCl}$ (containing $1.9 \mu \mathrm{M}$ porphyrin). The addition of $\mathrm{NaCl}$ induced no sigrificant spectral change (this is not shown). The titrant was then changed to NaOH containing $1.9 \mu \mathrm{M}$ porphyrin; the addition of base (indicated by heavy lines in the figure) shifted the equilibrium to total conversion to the free base. These experiments confirmed that the $435 \mathrm{~nm}$ band was the result of acid/ base chemistry, and emphasized the need for the presence of a pH buffer.

Experiments \#2-6: The Effect of Differing Experimental Conditions on the Beer's Law Plots. It was mentioned in the glassware cleaning section that the presence of alkali metal ions bound to the active sites on the glassware caused an uncontrolled variable in our experiments. In the course of the project we began to suspect that this variable was responsible for the loss in accuracy in our determination of the $\mathrm{H}_{2} \mathrm{TCPP}^{4-} / \mathrm{MV}^{2+}$ equilibrium constants. Another source of alkali metal ion concentration variability is the $\mathrm{KH}_{2} \mathrm{PO}_{4} / \mathrm{NaKHPO}_{4}$ buffer. As was described in the methods section, the pH of the buffer was adjusted to 7.0 with NaOH. Since we were adjusting the pH to a value very close to the second $\mathrm{pk}_{\mathrm{a}}$ of phosphate we would expect that the amount of sodium added would not be constant from experiment to experiment; as the concentration of buffer is 3 orders of magnitude greater than the porphyrin, the variability in the sodium ion concentration may be significant. What follows is a key to the figures and their corresponding experiment numbers from Table IX:

Figure $33 \mathrm{~A}$ (experiment 2 ) shows the linear range of the Beer's law plot and the porphyrin absorptivity for $\mathrm{H}_{2} \mathrm{TCPP}^{4-}$ under "ideal" conditions, i.e. high pH $(\approx 10)$, high temperature $\left(35^{\circ} \mathrm{C}\right)$, and low ionic strength $(\approx$ $5 e-4$ e.g. $0.5 \mathrm{mM} \mathrm{NaCl}$ ).

Figure 33B (experiment 3) shows the linear region of the Beer's law plot under "nearly ideal" conditions, identical to experiment 2 except for 
lower temperature $\left(20^{\circ} \mathrm{C}\right)$.

Figure 34A (experiment 4) shows the linear region of the Beer's law plot under the same conditions used in the $\mathrm{H}_{2} \mathrm{TCPP}^{4-} / \mathrm{viologen}$ experiments. The method utilized individually prepared solutions as described in method 1 of Chapter III $\left(5.0 \mathrm{mM} \mathrm{KH}{ }_{2} \mathrm{PO}_{4} / \mathrm{NaKHFO}_{4}, \mathrm{pH}=7.0, \mathrm{~T}=20^{\circ} \mathrm{C}\right)$.

Figure 34B (experiment 5) shows the results under identical conditions to experiment $4\left(5.0 \mathrm{mM} \mathrm{KH} \mathrm{PO}_{4} / \mathrm{NaKHPO}_{4}, \mathrm{pH}=7.0, \mathrm{~T}=20^{\circ} \mathrm{C}\right)$; however this experiment was performed as a titration (using method 2A, Table V in Chapter III). Fiqure 35 (experiment 6) shows the linear range of the Beer's law plots and the porphyrin absorptivity under "worst case" conditions, i.e. pH = 7. high ionic strength $(0.5 \mathrm{M} \mathrm{NaCl})$ and low temperature. Too few data points were taken at sufficiently low concentration to know if any linear region exists.

The results from this experimental series allowed us to define the conditions where anionic tetraphenylporphyrins exist in aqueous solution as the free base monomer (linear region of Beer's law). The source of the variability of the absorptivity determinations is investigated in the final section of this chapter.

SPECTRAL IINE SHAPE CHANGES INDUCED BY LOW BUFFER CONCENTRATION

In order to evaluate the $\mathrm{H}_{2} \mathrm{TCPP}^{4-} / \mathrm{viologen}$ complexation data by nonlinear least-squares analysis, the number of equilibrium equations must be known, i.e. least-squares methodology is "model-dependent". In order for the "model" to be meaningful, it was necessary to know if the porphyrin dimerization equation needed to be included. The linear region of the Beer's law experiments (Figure $34 \mathrm{~A}$ and B) showed $1 \mu \mathrm{M}$ $\mathrm{H}_{2} \mathrm{TCPP}^{4-}$ to be present only as a monomer under the conditions of $5 \mathrm{mM}$ phosphate and $20^{\circ} \mathrm{C}$. This allowed us to neglect the dimer equilibrium in

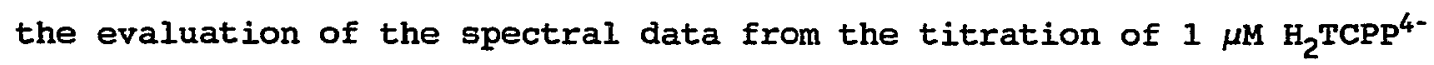
with $\mathrm{MV}^{2+}$ in $5 \mathrm{mM} \mathrm{KH} \mathrm{PO}_{4} / \mathrm{NaKHPO}_{4}, \mathrm{pH}=7.0$ and $\mathrm{T}=20^{\circ} \mathrm{C}$. However, 


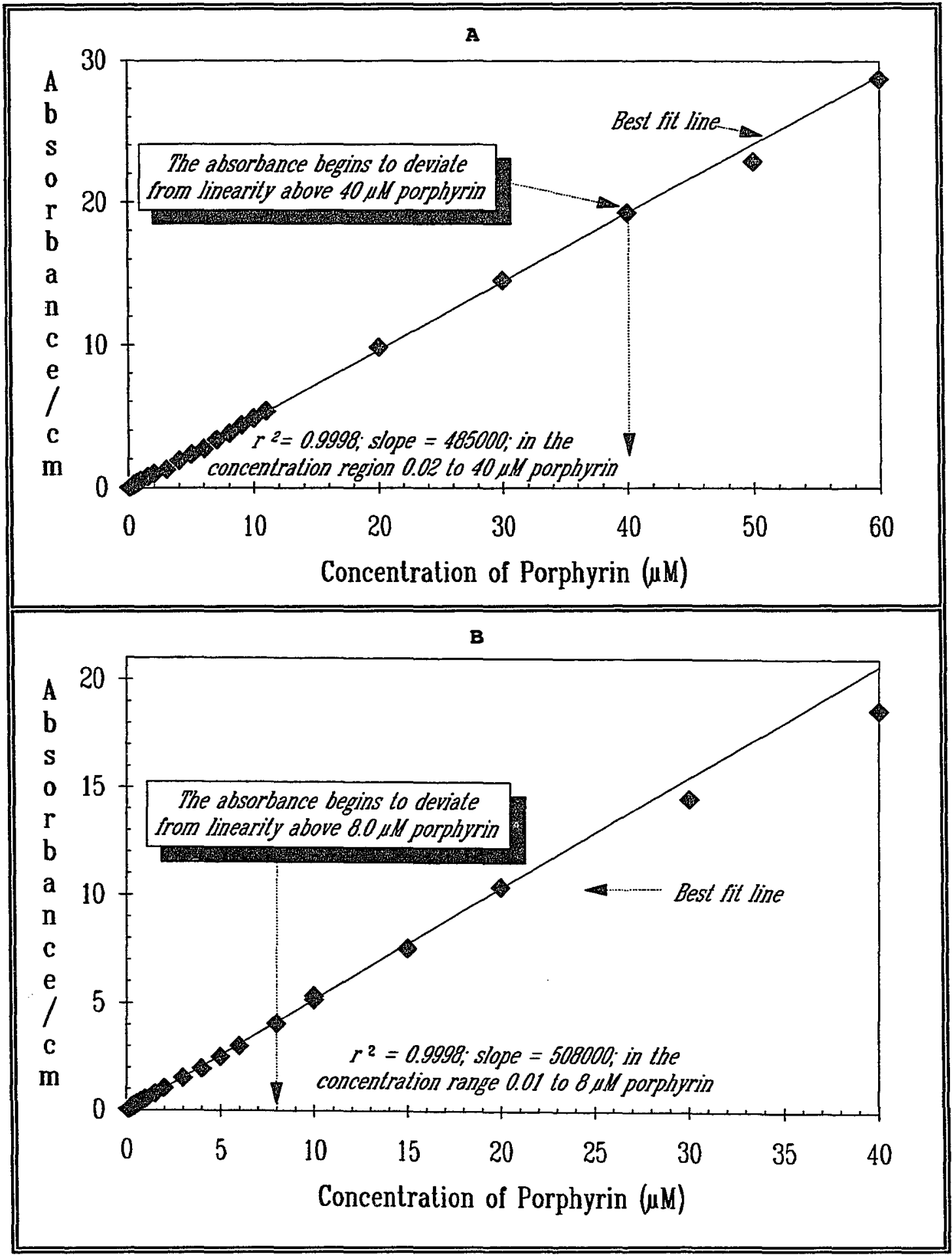

Figure 33. Beer's law plots of increasing concentration of H,TCPP $^{4-}$ at $\mathrm{pH}=10$ and minimal ionic strength. Absorbance normalized to $1 \mathrm{~cm}$ pathlength. "A": $T=35^{\circ} \mathrm{C}$ and "B": $T=20^{\circ} \mathrm{C}$. 


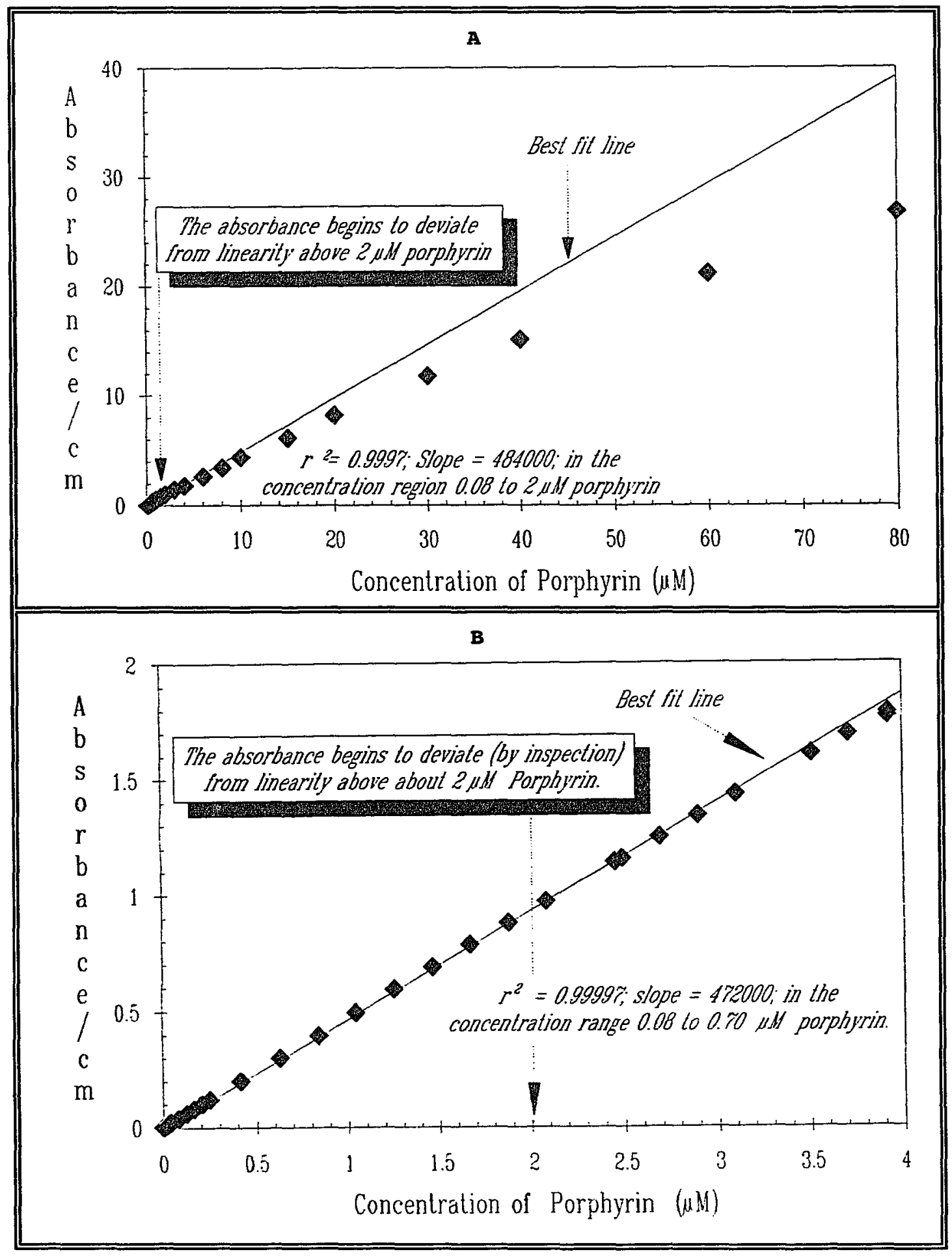

Figure 34. Beer's law plots of increasing concentration of H,TCPP $^{4-}$ at $\mathrm{pH}=7.0,5 \mathrm{mM}$ phosphate, $20^{\circ} \mathrm{C}$. Absorbance normalized to $1 \mathrm{~cm}$ pathlength ("A" variable "B" fixed). 


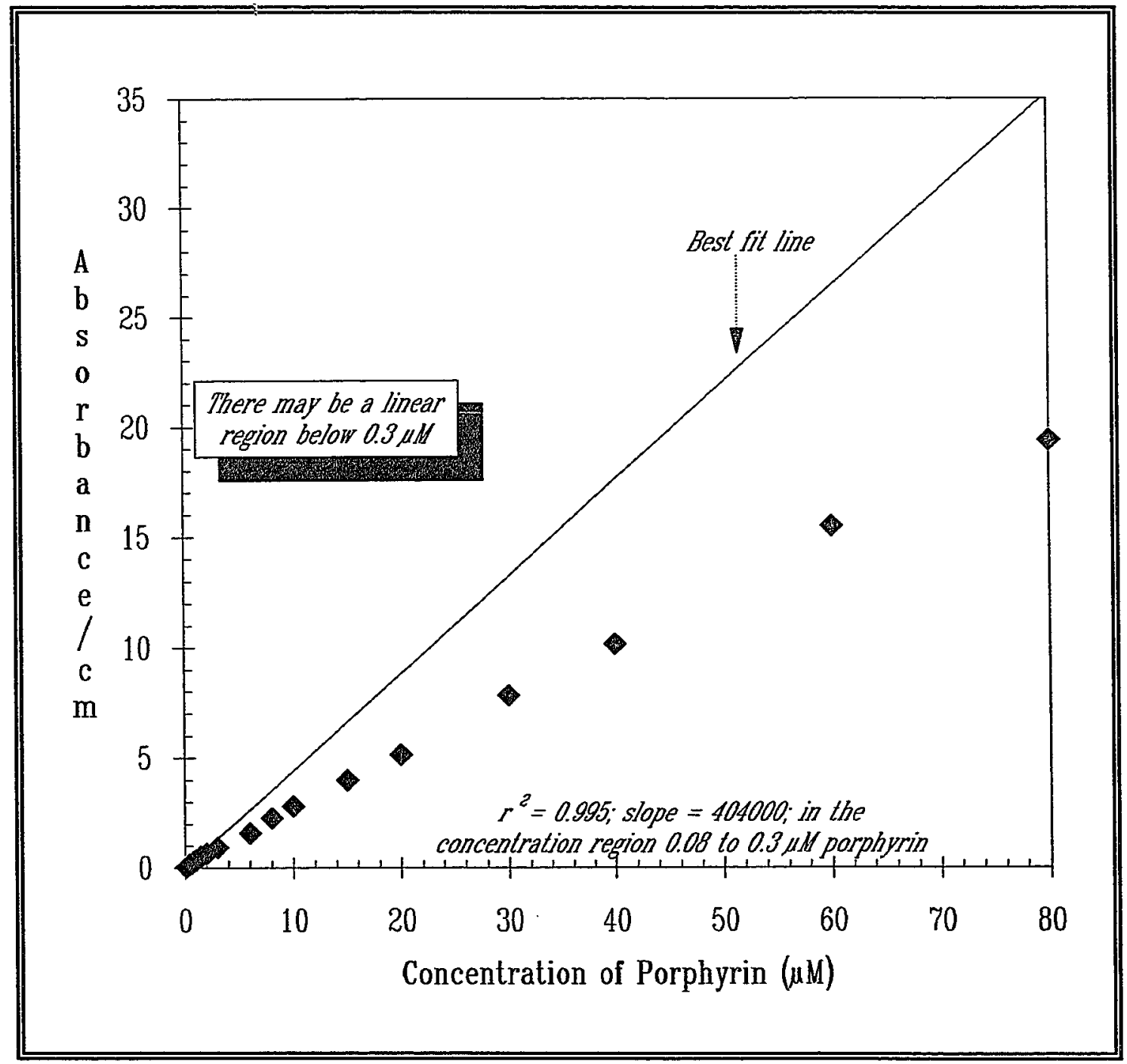

Fiqure 35. Beer's law plot of $\mathrm{H}_{2} \mathrm{TCPP}^{4-}$ at $\mathrm{pH} \approx 10, \mathrm{~T}=20^{\circ} \mathrm{C}$ and $0.50 \mathrm{M} \mathrm{NaCl}$.

because of the variance in the absorptivity measurements, it was not clear how best to determine the exact concentration of the porphyrin: either as the calculated concentration from the absorbance of the diluted stock solution and an absorptivity from the Beer's law experiments, or from the known dilution of the stock solution at known concentration. We chose the latter method since it was found that absorbance was not dependent upon porphyrin concentration alone (even at 
extremely dilute concentrations) but was dependent on the salt in solution.

The paragraph above outlines a strategy for evaluating spectral data performed at constant buffer concentrations; a strategy was needed for evaluating data under conditions of constant ionic strength, i.e. under variable salt conditions. We performed two such experiments (shown in chapter $V$ ) one using the buffer to maintain the ionic strength, and one using $\mathrm{KCl}$ to maintain the ionic strength. Focusing on the experiment using buffer to maintain the ionic strength, the initial concentration of phosphate buffer was $62 \mathrm{mM}$. The increased buffer concentration had a profound effect on both the absorptivity and the line shape of the soret band which is shown in Figure 36 below.

The body of porphyrin literature uniformly correlates such a change with the presence of porphyrin dimers in solution. However, nonlinear least-squares analysis of the titration data using the model of porphyrin dimerization plus the equations for porphyrin/viologen association gave unreasonable values for at least one of the parameters (for example, an absorptivity of one of the components as $1 \times 10^{-8} \mathrm{M}^{-1} \mathrm{~cm}^{-1}$ ) thereby indicating an incorrect model.

In an effort to understand the effect of buffer concentration (or alkali metal cation concentration) on the the porphyrin dimerization, we performed the control experiment which has been omitted in the literature; we evaluated the effect of salt concentration at constant porphyrin concentration within the linear range of the Beer's law plot. The unexpected results show the absorptivity of monomer to be a function of the buffer concentration. In Figure 38 we show the region in the buffer dependence experiment (Figure 37) at buffer concentrations below $5 \mathrm{mM}$ to show the initial region of the graph where the loss in absorbance may be due to the drop in $\mathrm{pH}$. Notice that the slope changes between approximately 0.2 and $0.6 \mathrm{mM}$ buffer.

The experiment was performed as a titration by method $2 \mathrm{~B}$ described 


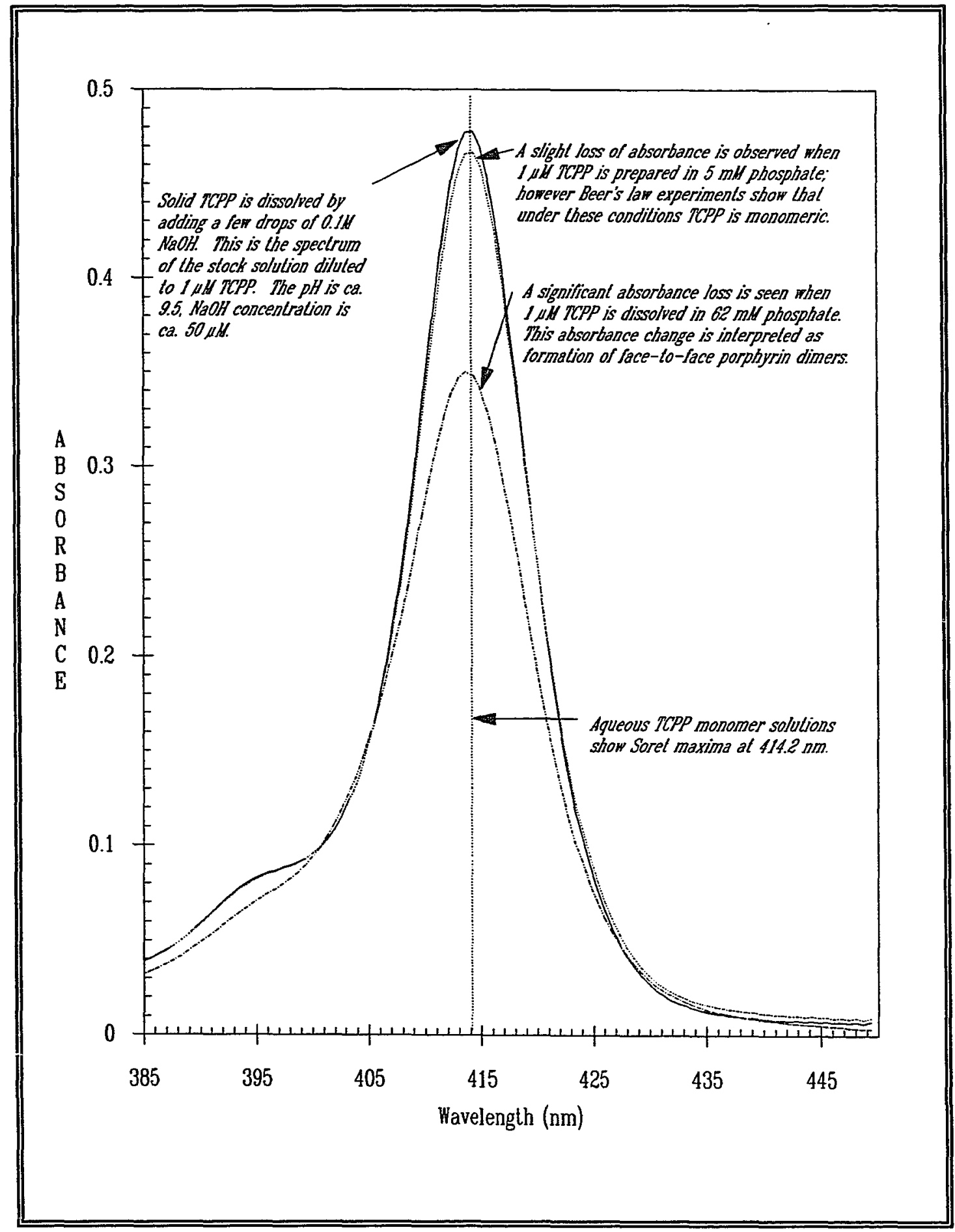

Figure 36. Comparison of the spectra of $1 \mu \mathrm{M} \mathrm{H}_{2} \mathrm{TCPP}^{4-} 0.5$ mM salt ( $\mathrm{pH} \approx 9.5), 5$ and $62 \mathrm{mM}$ phosphate ( $\mathrm{pH}=7.0$ ). TCPP $=\mathrm{H}_{2} \mathrm{TCPP}^{4}$, phosphate $=\mathrm{KH}_{2} \mathrm{PO}_{4} / \mathrm{NaKHPO}_{4}$. The three spectra are described by the annotation on the graph. 


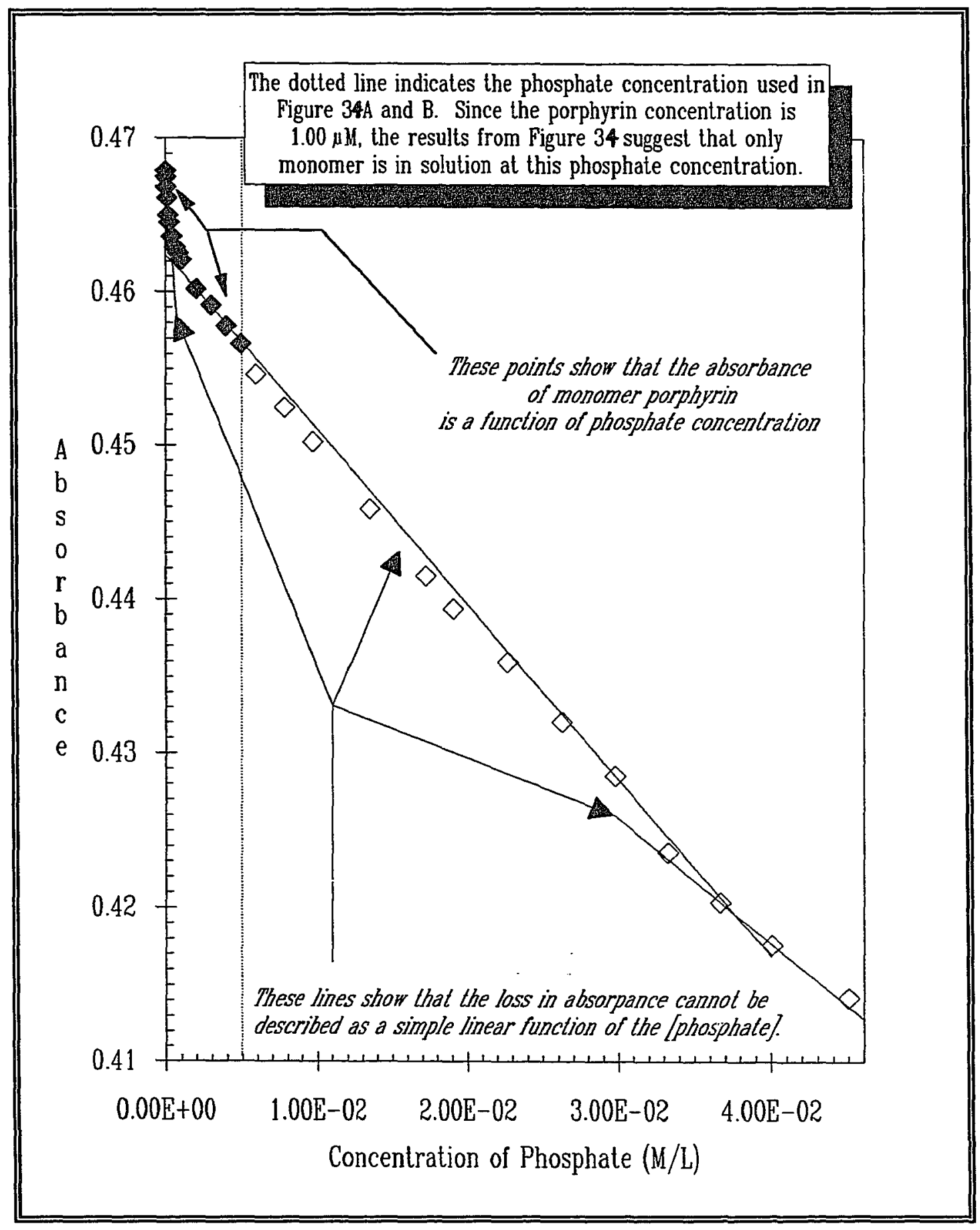

Figure 37. The effect of increasing phosphate concentration on the absorbance of $1.00 \mu \mathrm{M} \mathrm{B}_{2} \mathrm{TCPP}^{\mathrm{F}}$ at $414.2 \mathrm{~nm}$. The points indicate, respectively, the porphyrin absorbance at phosphate concentrations at or below (0), or above (0) that studied in the Beer's law experiments (Figure 34). The lines are included to show that the effect is not simple. 
in Chapter III. The porphyrin concentration was held constant in the experiment by adjusting the phosphate (buffer) titrant to $1 \mu \mathrm{M} \mathrm{H}_{2} \mathrm{TCPP}^{4-}$. The ionic strength of an aliquot of the $\mathrm{H}_{2} \mathrm{TCPP}^{4-}$ solution was increased with NaCl before the initial $\mathrm{pH}$ was measured.

The results from this experiment explain the disagreement between

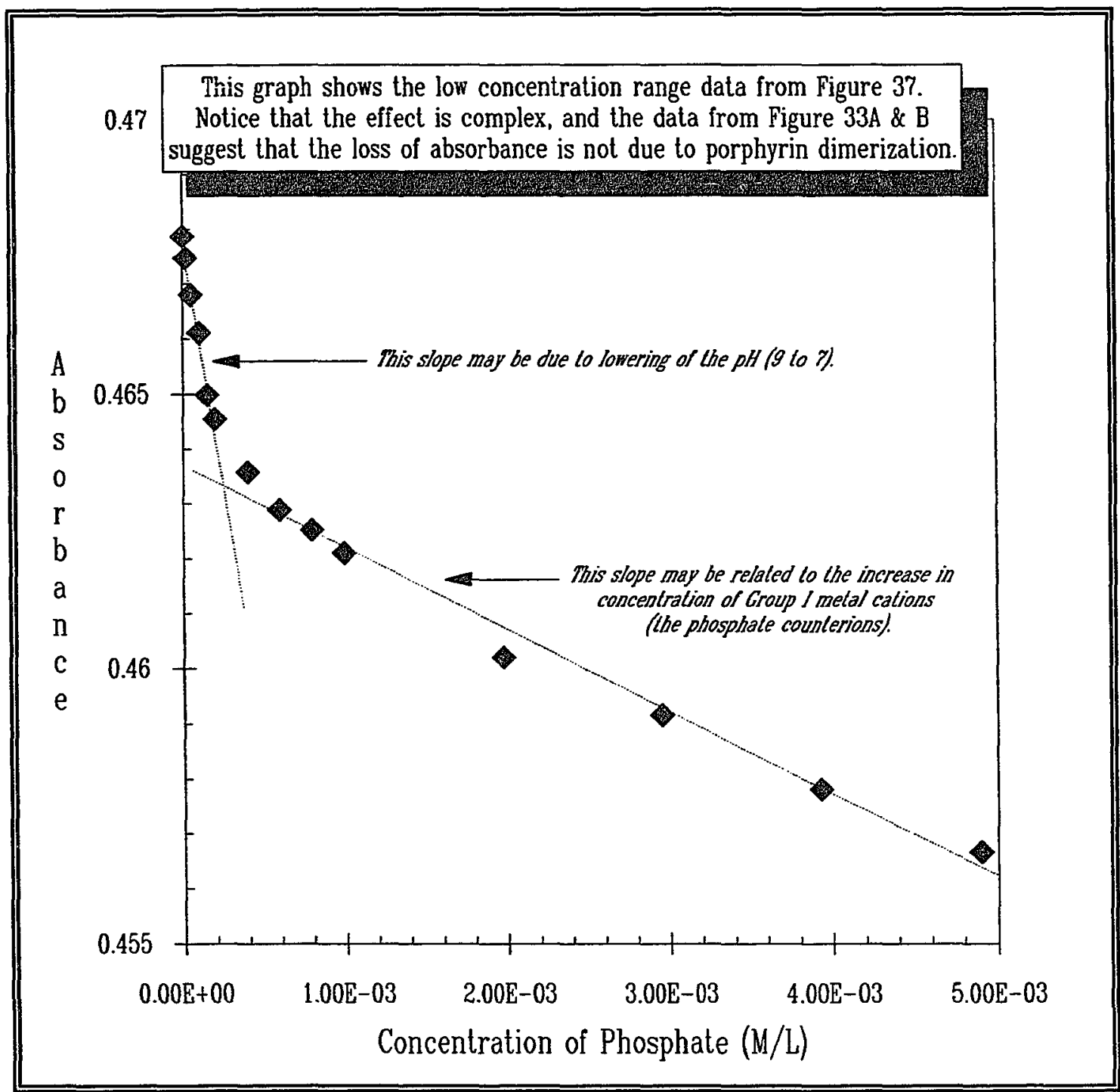

Figure 38. The effect of low phosphate concentration on the E $_{2}$ TCPP ${ }^{4-}$ absorbance at $414.2 \mathrm{~nm}$. This graph shows the low concentration region described in Figure 37 . The concentration of $\mathrm{B}_{2} \mathrm{TCPP}^{4^{-}}=1 \mu \mathrm{M}$, the initial concentration of $\mathrm{NaOH} \approx 0.5 \mathrm{mM}$ and the initial $\mathrm{pH}=9$. We expect the $\mathrm{pH}$ at $1 \mathrm{mM}$ phosphate to be approaching 7 . 
the precision and accuracy in the Beer's law plots, especially those performed at $5 \mathrm{mM}$ phosphate (Figure 34, experiments 4 and 5). It was explained previously that the alkali metal concentration varied from experiment to experiment due to variabilities inherent in the pH adjustment and glassware treatment. This may also explain the variability in the reported absorptivities for various porphyrins in the literature.

By inspection of the spectral change induced by $62 \mathrm{mM}$ buffer, as shown in Figure 36, we noticed that the drop in absorbance was accompanied by a broadening in the peak. Evaluation of the absorptivity for a single solution species by using the absorbance at the wavelength of the peak maximum is only valid in the absence of spectral line shape change. The more rigorous Beer's law equation uses the area under the peak. We calculated the area under the curve by first plotting the data shown in Figure 37 on an energy scale, decomposing each spectrum into its Gaussian components (plus an energy baseline) and calculating the total area as the sum of the Gaussian components (Gaussian decompositions done by Harry Bell). The sum of the Gaussians and the Gaussian decompositions are shown in Figure 39; the relative areas are plotted in Figure 40. In the buffer concentration region from 0 to 44 $\mathrm{mM}$, the absorbance at the wavelength maximum was reduced by approximately $13 \%$ compared to a $7 \%$ drop in the integrated absorbance. Correlating the results with a specific solution phenomenon is hampered by the numerous phenomena which are known to induce spectral line shape changes (inhomogeneous broadening, collisional broadening, pressure broadening etc.) (149-152). It also appears that the decrease in area does not unambiguously indicate a specific phenomenon, such as complexation.

Although this phenomenon has not been reported for porphyrins, similar results have recently been reported for the xanthene dye, rose bengal (147). The authors were always able to find an alkali metal ion 


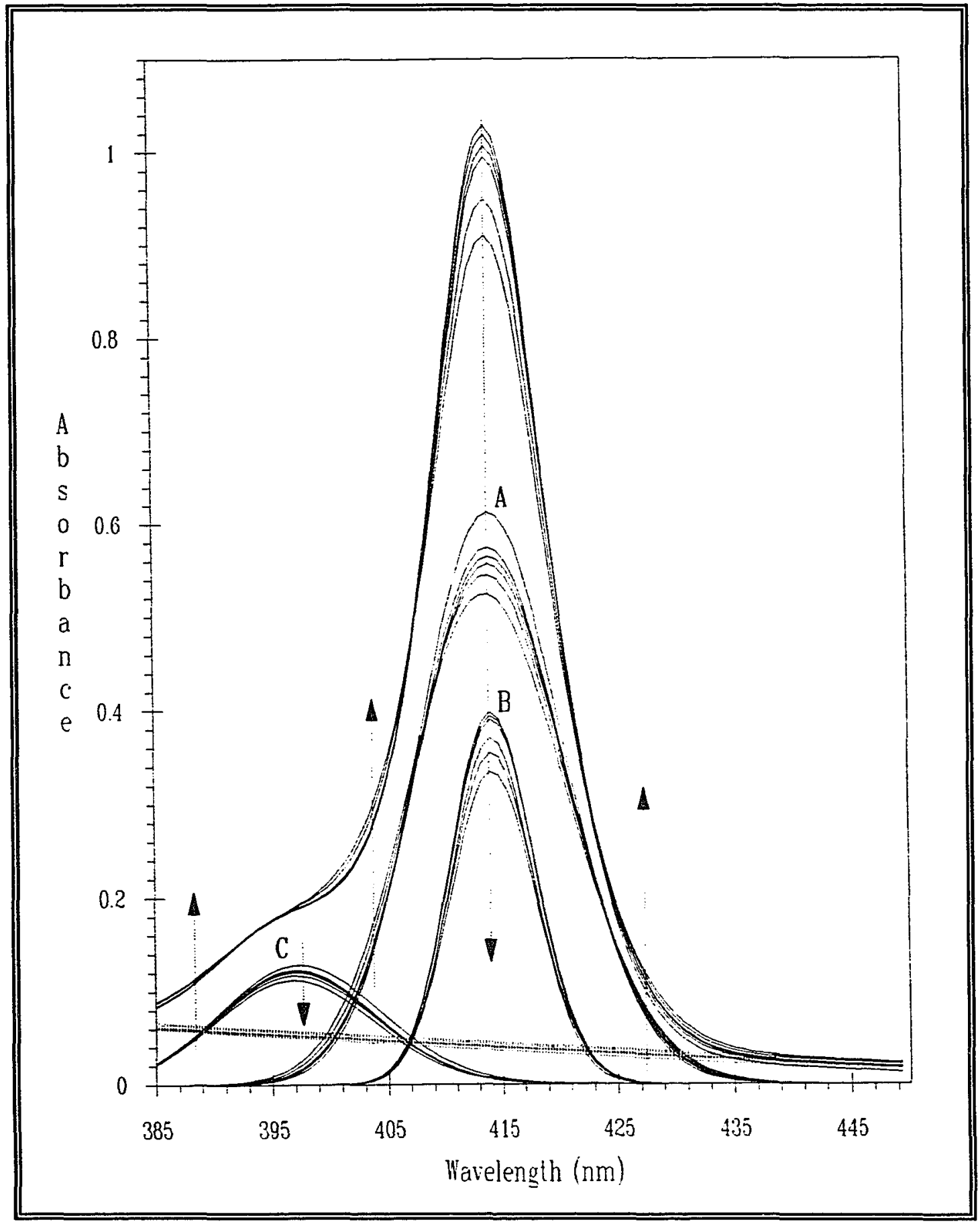

Figure 39. Gaussian decomposition of the phosphate dependence spectral data. Every fourth spectrum is shown from the data set in Figure 37. The highest curve shows the sum of $A, B$ and $C$. The arrows indicate the spectral changes

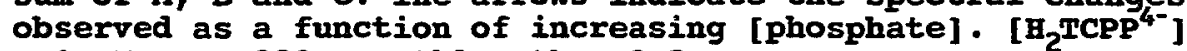
$=1 \mu \mathrm{M}, \mathbf{T}=20^{\circ} \mathrm{C}$, pathlength $=2.2 \mathrm{~cm}$. 


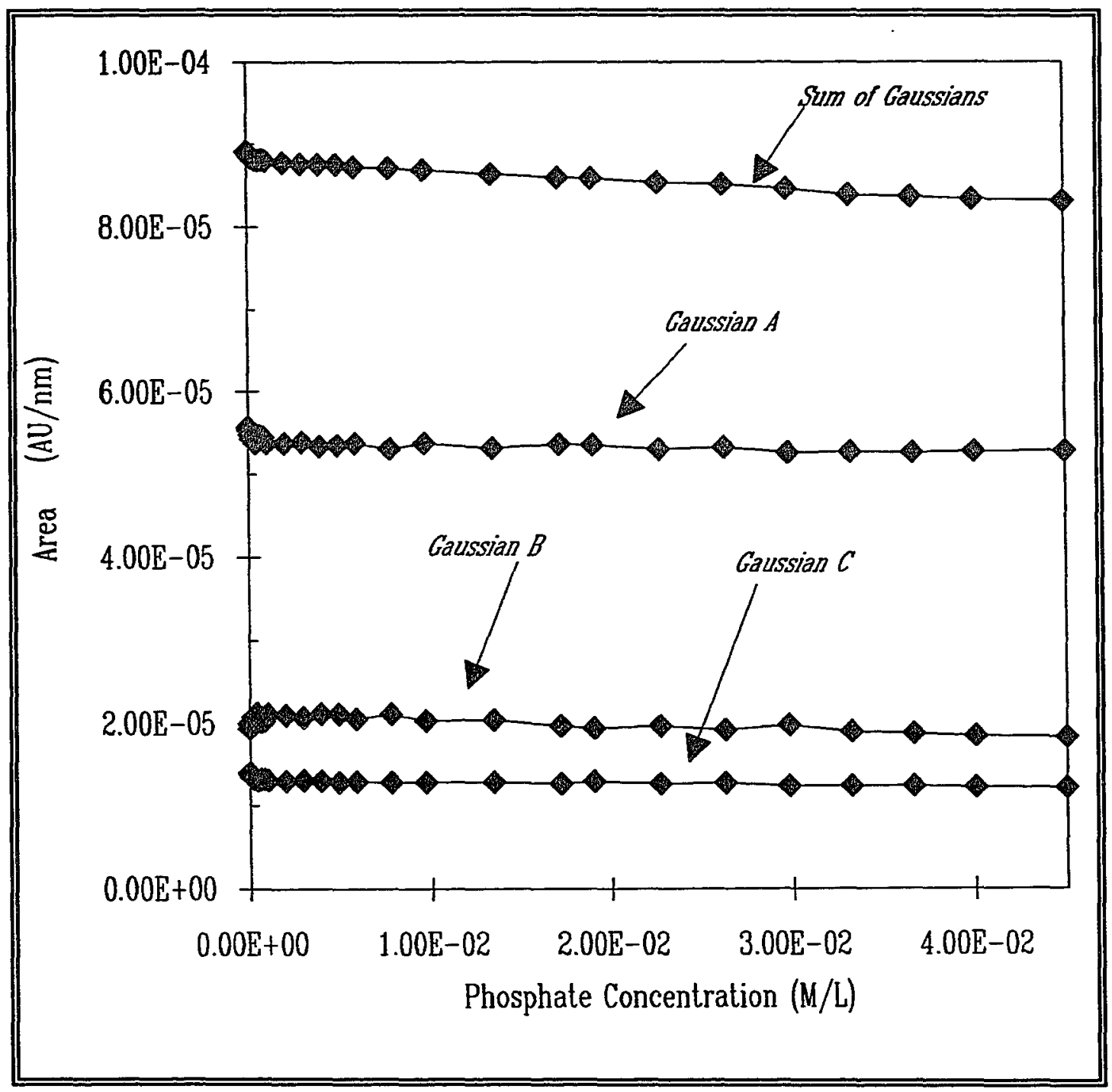

Figure 40. Plot of the integrated decomposed Gaussian absorbance components from Figure 39 versus phosphate concentration. $A, B$, and $C$ refer to the Gaussian decompositions shown in Figure 39.

concentration region, below the concentration which yielded the typical dimer spectrum, where the absorptivity of the monomer was a function of the cation concentration. Moreover, this effect was specific to the alkali metal cation species studied, the greatest effect produced by the ion with the highest charge-to-radius ratio (the most polarizing cation). The authors attributed this effect to chelation (or 
association) of the cation by the dye.

Unlike free base porphyrins, xanthene dyes lack a multidentate chelation site (although there is the possibility of ion-pair formation in rose bengal as in the anionic tetraphenylporphyrins). Since alkali metal chelation in neutral aqueous solution has been investigated for free base porphyrins with negative results (121), chelation is even less probable for the xanthene dyes. It may be that the transition dipole moment is affected by the proximity of polarizing alkali metal cations for these extremely sensitive oscillators. Cation-induced porphyrin spectral line shape changes cause serious complications with respect to the utilization of absorbance data to calculate association constants; this subject is discussed further in the following chapter. 
CEAPTER $v$

DETERMIMATION OF THE STOICEIOMETRY AND ASSOCIATION CONSTANTS FOR THE COMPLFXATION OF $\mathrm{H}_{2} \mathrm{TCPP}^{4-}$ WITH $\mathrm{MV}^{2+}$

\section{INTRODUCTION}

In Chapter IV we presented qualitative evidence which allowed us to correlate the spectral changes observed in the titration of $\mathrm{H}_{2} \mathrm{TCPP}^{4-}$ with viologens to specific porphyrin species in aqueous buffered solution at neutral $\mathrm{pH}$ values. In summary, $1 \mu \mathrm{M} \mathrm{H}_{2} \mathrm{TCPP}^{4-}$ exists only in monomer form with the $\lambda_{\max }$ of its soret band at $414.2 \mathrm{~nm}$ and its absorptivity between $480,000 \pm 10,000 \mathrm{M}^{-1} \mathrm{~cm}^{-1}$ under the following conditions: $5 \mathrm{mM}$ phosphate, $\mathrm{pH}=7.0$, and $\mathrm{T}=20^{\circ} \mathrm{C}$. The reason for the large uncertainty in the absorptivity of the monomer is due to its strong dependence upon the alkali metal ion concentration $\left(\mathrm{KH}_{2} \mathrm{PO}_{4} / \mathrm{NaKHPO}_{4}\right)$. Therefore, the loss of absorbance observed upon increased buffer concentration (between 2 and $40 \mathrm{mM}$ ) was explained not by dimer formation, but most plausibly by an unexplained sensitivity of the monomer absorptivity to the presence of strongly polarizing (high charge-to-radius ratio) inorganic salts. This information was helpful in developing an appropriate set of equilibrium equations to model titration experiments (porphyrin + viologen) performed at buffer concentrations higher than $5 \mathrm{mM}$ as we were led to question the presence of the monomer/dimer equilibrium.

The $\mathrm{H}_{2} \mathrm{TCPP}^{4-}$ dimer spectrum exhibited a severely weakened and blue-shifted soret band relative to the monomer spectrum. The Soret was shifted by approximately 7 to $10 \mathrm{~nm}$ depending upon the conditions used to induce the dimer. At high alkali metal ion concentration $(\approx 3 \mathrm{M}$ NaCl, Figure 28) the dimer soret band was broader and less blue-shifted 
relative to the dimer spectra induced by organic salts such as PVs ${ }^{0}$ or CTAB (Figures 23 and 26).

When $\mathrm{H}_{2} \mathrm{TCPP}^{4-}$ was complexed by $\mathrm{MV}^{2+}$ or $\mathrm{PVS}^{0}$, the soret band was progressively weakened and red-shifted as a function of increasing viologen concentration. Therefore, the complexation could be quantified by correlating the spectral changes with the viologen concentration (at constant porphyrin concentration). The quantification was more difficult when the complexation was studied at constant ionic strength due to the dependence of porphyrin absorptivity upon both the buffer and viologen concentration.

Figure 36 illustrated the spectral change induced by 5 versus 62 MM phosphate (the initial concentration of buffer in the titration performed at constant ionic strength). Analysis of the data from experiments at constant ionic strength required a method which was "model independent" $i . e$. where the number of equilibrium equations was not specified, to allow the possibility of the presence of the porphyrin dimer, or some other unforeseen equilibrium. We developed a method using principal component analysis (104-112) to analyze these data. This method (described in chapter III) yields information concerning the number of principal components in solution (the number of absorbing species) which could be used to determine the number of equilibrium equations; in addition, under conditions where only two components are in solution (viologen concentration regions in the titration where an isosbestic point is observed), this method may be used to determine the spectral line shape and absorptivity of the individual components. This is very useful when one of the components is not resolved, as in the case of the porphyrin:viologen 1:1 complex shown in Figures 23 and 4143.

In the following section we present a table summarizing the published values for porphyrin/viologen association constants followed by a detailed review of seven publications $(44,47,48,52,53,55,57)$ which 
study tetraphenylporphyrin/viologen complexation. In order to put the work by these authors in perspective, it should be mentioned that all of these studies were "goal oriented", the goal being to optimize photocatalyzed charge transfer. This explains why fluorescence or lifetime methods were used and why the authors did not study the effect of very high or very low viologen concentrations. Our data and data analysis follow this review.

\section{REVIEW OF THE LITERATURE}

The critical portion of this review is limited to the use of UV/VIS absorbance spectral data to quantify association constants. It is interesting that in general the authors relied on less direct

TABLE $\mathbf{X}$

SUMMARY OF REPORTED EQUIIIBRIUM CONSTANTS

\begin{tabular}{|c|c|c|c|c|}
\hline Porphyrin & viologen & $\mathbf{K}_{\text {eq }}$ & [Salt] & $\underset{N}{\text { Reference }}$ \\
\hline ZnTSPP ${ }^{4-}$ & $\mathrm{MV}^{2+}$ & 9,300 & 0 & 56 \\
\hline ZnTSPP ${ }^{4-}$ & $\mathrm{MV}^{2+}$ & 15,000 & $\approx 0$ & 45 \\
\hline ZnTSPP ${ }^{4-}$ & $\mathrm{MV}^{2+}$ & 5,200 & $1 \mathrm{mM} \mathrm{KCl}$ & 54 \\
\hline ZnTSPP ${ }^{4-}$ & $\mathrm{MV}^{2+}$ & 1,600 & $100 \mathrm{mM} \mathrm{KCl}$ & 45 \\
\hline ZnTSPP ${ }^{4-}$ & $\mathrm{MV}^{2+}$ & 1,500 & $100 \mathrm{mM}$ Phosphate & 46 \\
\hline $\mathrm{ZnTS}_{3} \mathrm{PP}^{3-}$ & $\mathrm{MV}^{2+}$ & $2,100(?)$ & $20 \mathrm{mM}$ TRIS + $\mathrm{KCl} *$ & 51 \\
\hline $\mathrm{ZnTS}_{3} \mathrm{PP}^{3-}$ & $\mathrm{MV}^{2+}$ & 1,700 & $200 \mathrm{mM}$ TRIS + NaCl* & 57 \\
\hline $\mathrm{H}_{2} \mathrm{TSPP}^{4-}$ & $\mathrm{MV}^{2+}$ & 647 & $50 \mathrm{mM} \mathrm{NaCl}$ & 44 \\
\hline $\mathrm{H}_{2} \mathrm{TS}_{3} \mathrm{PP}^{3-}$ & $\mathrm{MV}^{2+}$ & 810 & $50 \mathrm{mM}$ Phosphate & 52 \\
\hline $\mathrm{H}_{2} \mathrm{TS}_{3} \mathrm{PP}^{3-}$ & $\mathrm{MV}^{2+}$ & 170 & $1,200 \mathrm{mM} \mathrm{NaCl}$ & 52 \\
\hline ZnTSPP $^{4-}$ & PVS $^{0}$ & 3,000 & $\approx 0$ & 45 \\
\hline ZnTSPP ${ }^{4-}$ & PVS $^{0}$ & 15 & $1 \mathrm{mM} \mathrm{KCl}$ & 54 \\
\hline ZnTSPP ${ }^{4-}$ & PVs $^{0}$ & 700 & $100 \mathrm{mM} \mathrm{KCl}$ & 45 \\
\hline $\mathrm{ZnTS}_{3} \mathrm{PP}^{3-}$ & PVS $^{0}$ & 730 & $200 \mathrm{mM}$ TRIS + NaCl* & 57 \\
\hline
\end{tabular}

* Indicates that the experiment was performed at constant ionic strength. 
methods, such as fluorescence quenching, to examine porphyrin/viologen association. This probably explains why the stoichiometries were not determined, although it appeared to be of considerable interest to the various authors whose work is reviewed here. Methods such as fluorescence quenching or lifetime studies are mentioned only briefly, although these methods may comprise the central experimental method.

In 1981 schmehl and whitten (44) investigated the complexation of $\mathrm{H}_{2} \mathrm{TSPP}^{4-}$ (and PdTSPP ${ }^{4-}$ ) by $\mathrm{MV}^{2+}$ and benzyl viologen $\left(\mathrm{BV}^{2+}\right.$ ) in unbuffered aqueous solution at $25^{\circ} \mathrm{C}$ containing $50 \mathrm{mM}$ NaCl. They were unable to quantify porphyrin:viologen association constants from their UV/VIS absorbance data using a linearization method (113) but did report constants estimated from fluorescence quenching data:

$$
\begin{aligned}
& \mathrm{H}_{2} \mathrm{TSPP}^{4-}+\mathrm{MV}^{2+} \rightarrow \mathrm{H}_{2} \mathrm{TSPP}^{4-}-\mathrm{MV}^{2+} \quad \mathrm{K}_{\mathrm{eq}}=647 \mathrm{M}^{-1} \\
& \mathrm{H}_{2} \mathrm{TSPP}^{4-}+\mathrm{BV}^{2+} \leftarrow \mathrm{H}_{2} \mathrm{TSPP}^{4-}-\mathrm{BV}^{2+} \quad \mathrm{K}_{\mathrm{eq}}=4,783 \mathrm{M}^{-1}
\end{aligned}
$$

Studies both by us and by Wilkins and coworkers (69) have shown that the complexation behavior of the two anionic tetraphenylporphyrins, $\mathrm{H}_{2} \mathrm{TCPP}^{4-}$ and $\mathrm{H}_{2} \mathrm{TSPP}^{4-}$ are completely analogous; therefore we would predict that the association constants for $\mathrm{H}_{2} \mathrm{TSPP}^{4-}$ with $\mathrm{MV}^{2+}$ should be very close to those determined by us for $\mathrm{H}_{2} \mathrm{TCPP}^{4-}$ with $\mathrm{MV}^{2+}$ (Table XI). The low values reported by Schmehl and whitten are probably due to the high electrolyte concentration. These authors attempted to correlate electrochemical and photochemical studies, which explains the presence of electrolyte and illustrates the complications encountered in electrochemical studies of porphyrins in aqueous solution (salt must be present as a supporting electrolyte). In the course of their very useful study, they performed preliminary investigations of the effect of other electrolytes, such as the alkylammonium salts, and found results similar to ours (reported in Chapter IV).

In 1982 Richoux and Harriman (48) developed a rationale for choosing the specific substituted zinc tetraphenylporphyrin that would yield maximal charge transfer to $\mathrm{Mv}^{2+}$ in solution. They showed that a 
favorable (more negative) porphyrin reduction potential was not as important as minimal complexation by considering the rate of electron transfer versus the rate of dissociation of the charge transfer pair. However, they estimated the strength of the porphyrin/Mv ${ }^{2+}$ association complexes solely on the basis of coulombic attraction, which later studies by other authors showed to be inaccurate in most cases.

Richoux and Harriman reasoned that cationic zinc tetraphenylporphyrins have unfavorable reduction potentials, but should not complex with $\mathrm{MV}^{2+}$ and therefore should give larger quantum yields for reduced viologen. They concluded that although $\mathrm{ZnTCPP}^{4-}$ had the most favorable reduction potential, it would give the lowest quantum yield for (net) reduced $\mathrm{MV}^{2+}$ due to the formation of (relatively) strong association complexes. In addition, Richoux and Harriman addressed the question of how to take advantage of the very favorable reduction potentials of the anionic tetraphenylporphyrins and suggested the strategy of using porphyrins with lower net negative charge (the trisubstituted anionics, e.g. $\mathrm{ZnTS}_{3} \mathrm{PP}^{3-}$ ). Another strategy discussed was to change the charge characteristics of the viologen, which led to the interest in the zwitterionic viologen, $\mathrm{PVS}^{0}$.

In 1983 kano et al. (52) found that $\mathrm{H}_{2} \mathrm{TS}_{3} \mathrm{PP}^{3-}$ formed ground-state association complexes with both positively charged methyl viologen and negatively charged anthraquinone disulfonate (AQDS ${ }^{2-}$ ). They calculated association constants from both UV/VIS absorbance and fluorescence quenching data in aqueous solution at $25^{\circ} \mathrm{C}$ buffered to $\mathrm{pH}=8.0$ with 50 $\mathrm{mM}$ phosphate $\left(\left[\mathrm{H}_{2} \mathrm{TS}_{3} \mathrm{PP}^{3-}\right]=25 \mu \mathrm{M}\right)$ using a linearization method (113).

$$
\begin{aligned}
& \mathrm{H}_{2} \mathrm{TS}_{3} \mathrm{PP}^{3-}+\mathrm{MV}^{2+}-\mathrm{H}_{2} \mathrm{TS}_{3} \mathrm{PP}^{3-}-\mathrm{MV}^{2+} \mathrm{K}_{\text {eq(Abs })}=810 ; \mathrm{K}_{\text {eq(Fl) }}=740 \mathrm{M}^{-1} \\
& \mathrm{H}_{2} \mathrm{TS}_{3} \mathrm{PP}^{3-}+\mathrm{AQDS}^{2-} \Rightarrow \mathrm{H}_{2} \mathrm{TS}_{3} \mathrm{PP}^{3-}-\mathrm{AQDS}^{2-} \mathrm{K}_{\text {eq(Abs })}=4300 ; \mathrm{K}_{\text {eq(Fl }}=4600 \mathrm{M}^{-1}
\end{aligned}
$$

When the experiments were repeated in solutions containing $1.2 \mathrm{M}$ NaCl the authors calculated the following association constants from their fluorescence data: 


$$
\begin{aligned}
& \mathrm{H}_{2} \mathrm{TS}_{3} \mathrm{PP}^{3-}+\mathrm{MV}^{2+} \Rightarrow \mathrm{H}_{2} \mathrm{TS}_{3} \mathrm{PP}^{3-}-\mathrm{MV}^{2+} \quad \mathrm{K}_{\text {eq(Fl })} 170 \mathrm{M}^{-1} \\
& \mathrm{H}_{2} \mathrm{TS}_{3} \mathrm{PP}^{3-}+\mathrm{AQDS}^{2-} \Rightarrow \mathrm{H}_{2} \mathrm{TS}_{3} \mathrm{PP}^{3-}-\mathrm{AQDS}^{2-} \quad \mathrm{K}_{\text {eq(Fl) }} 7800 \mathrm{M}^{-1}
\end{aligned}
$$

It is interesting that these authors report a greater than $1: 1$ stoichiometry (but were unable to quantify the higher stoichiometry) for the $\mathrm{ZnTS}_{3} \mathrm{PP}^{3-}: \mathrm{ADQS}^{2-}$ system from fluorescence quenching data, but report a 1:1 stoichoimetry for $\mathrm{ZnTS}_{3} \mathrm{PP}^{3-}: \mathrm{MV}^{2+}$ (the author suspects a stoichiometry of $1: 2$ for the $\mathrm{ZnTS}_{3} \mathrm{PP}^{3-}: \mathrm{MV}^{2+}$ system by analogy to our data for $\mathrm{H}_{2} \mathrm{TCPP}^{4-}: \mathrm{MV}^{2+}$ complexation). Complex formation resulted in static quenching of the porphyrin fluorescence in both examples. ("Static quenching" describes the observation that fluorescence quenching occurs at a rate faster than diffusion, implying the presence of association complexes.) They concluded that hydrophobic effects explained the higher association constants for the $\mathrm{ADQS}^{2-} / \mathrm{H}_{2} \mathrm{TS}_{3} \mathrm{PP}^{3-}$ system by studying the temperature and salt dependence of the association complexes. The $\mathrm{MV}^{2+}$ concentrations studied by Kano et al. were: $0.07,0.29$, 0.48 , and $0.8 \mathrm{mM}$. By analogy to our data these authors studied the formation of the 1:1 porphyrin:viologen complex. The salt concentration used in this experiment (50 mM phosphate) does not explain the low association constants observed by these authors (Table $\mathrm{X}$ and XII). In 1984 Sato, Ogawa and Kano (53) extended the previous study to include nine additional anionic aromatic salts. They correlated the net charge with the magnitude of the association constants. Of interest was the study of $\mathrm{H}_{2} \mathrm{TS}_{3} \mathrm{PP}^{3-} /$ quencher association constants by comparing the quencher, $A D Q S^{2-}$, with its analogue $A Q B S^{1-}$. The decrease in negative charge reduced the coulombic repulsion between the porphyrin and the quencher resulting in a doubling of the association constant.

In 1985 Aono and Okura (57) showed that the efficiency of charge separation was improved by increasing the electrostatic repulsion between porphyrin and acceptor by using a neutral zwitterionic viologen $\left(\mathrm{PVS}^{0}\right)$ that becomes negatively charged after reduction. They compared the $\mathrm{ZnTS}_{3} \mathrm{PP}^{3-} / \mathrm{MV}^{2+}$ system with the $\mathrm{ZnTS}_{3} \mathrm{PP}^{3-} / \mathrm{PVS}^{0}$ system and found that 
the quantum yield for charge separation was dependent upon the viologen concentration and was larger for $\mathrm{PVS}^{0}$ than for $\mathrm{MV}^{2+}$. In addition, the greater reduction rate of $\mathrm{PVS}^{0}$ under steady-state irradiation was explained by finding that the complexation constant for $\mathrm{ZnTS}_{3} \mathrm{PP}^{3-} / \mathrm{PVS}^{0}$ was smaller than that for $\mathrm{ZnTS}_{3} \mathrm{PP}^{3-} / \mathrm{Mv}^{2+}$. Aono and Okura concluded that only the uncomplexed $\mathrm{ZnTS}_{3} \mathrm{PP}^{3-}$ acts as a photosensitizer.

These authors studied association in aqueous solution by UV/VIS absorbance spectroscopy in both the soret and Q-band region but did not use these data to determine association constants. The conditions used were $\mathrm{pH}=7.0$ (adjusted with $200 \mathrm{mM}$ TRIS buffer, $\mathrm{pk}_{\mathrm{a}}=8.2$, therefore they adjusted the $\mathrm{pH}$ nearly out of the buffering range), $\mathbf{T}=25^{\circ} \mathrm{C}$, and constant ionic strength adjusted with Nacl. Their titration spectral data in the soret region resemble our data at constant ionic strength where the initial $\mathrm{KCl}$ concentration $=1.5 \mathrm{M}$ (Figure 46$)$. They calculated association constants by a linearization method from data obtained by a complex technique using flash photolysis:

$$
\begin{array}{ll}
\mathrm{ZnTS}_{3} \mathrm{PP}^{3-}+\mathrm{MV}^{2+}-\mathrm{ZnTS}_{3} \mathrm{PP}^{3-}-\mathrm{MV}^{2+} & \mathrm{K}_{\mathrm{eq}}=1,700 \mathrm{M}^{-1} \\
\mathrm{ZnTS}_{3} \mathrm{PP}^{3-}+\mathrm{PVS}^{0}-\mathrm{ZnTS}_{3} \mathrm{PP}^{3-}-\mathrm{PVS}^{0} & \mathrm{~K}_{\mathrm{eq}}=730 \mathrm{M}^{-1}
\end{array}
$$

In 1985 Nahor and Rabani (56) studied the association of $\mathrm{ZnTSPP}^{4-}$ with $\mathrm{MV}^{2+}$. These authors calculated association constants from fluorescence data, then used the simple Debye-Hückel equation to calculate a constant at zero ionic strength.

$$
\mathrm{ZnTSPP}{ }^{4-}+\mathrm{MV}^{2+} \rightarrow \mathrm{ZnTSPP}^{4-}-\mathrm{MV}^{2+} \mathrm{K}_{\mathrm{eq}(\mathrm{Fl})} 9,300 \mathrm{M}^{-1}
$$

Porphyrins are neither point charges nor spherical, conditions for description of an ion by this theory. In light of our results on the dependence of the absorptivity on the alkali cation concentration, and the nature of the porphyrin anion, we question if the extrapolation to zero ionic strength using the Debye-Hückel equation is valid.

In 1987 Kano, Nakajima, and Hashimoto (58) studied the fluorescence quenching of a cationic porphyrin, $\mathrm{H}_{2} \mathrm{TMPy}^{4+}$, by both cationic and anionic aromatic molecules. This porphyrin is unique as it 
has been shown to exist in dimer form in aqueous solutions in concentrations as low as $0.1 \mu \mathrm{M}(58,66)$. The authors demonstrated via $400 \mathrm{MHz}^{1} \mathrm{H}$ NMR that, analogous to the anionic tetraphenylporphyrins, $\mathrm{H}_{2}$ TMPYP $^{4+}$ can form association complexes with organic molecules of like or opposite charge.

They suspected higher stoichiometries for complexation of $\mathrm{H}_{2}$ TMPYP $^{4+}$ by the anionic dye, AQßS ${ }^{1-}$, but were unable to determine the number of AQßS ${ }^{1-}$ ions associated with the $\mathrm{H}_{2} \mathrm{TMPY}^{4+}$ dimer.

Kano et al. used UV/VIS absorbance spectroscopy to demonstrate the existence of an isosbestic point in the spectral study of $\mathrm{H}_{2}$ TMPYP $^{4+}(100$ $\mu \mathrm{M})$ upon addition of $0,5,10,15,20,25,30$, and $35 \mathrm{mM} \mathrm{3,6-}$ dimethylacridinium cation $\left(\mathrm{PFL}^{1+}\right)$. However, they were unable to calculate association constants from these data by either of two linearization methods attempted $(113,114)$.

\section{DETERMINATION OF ASSOCIATION CONSTANTS}

Our work was centered around the determination of association constants for titration of $\mathrm{H}_{2} \mathrm{TCPP}^{4-}$ with $\mathrm{MV}^{2+}$ from $\mathrm{UV} / \mathrm{VIS}$ absorbance data. We will show how powerful such studies can be when combined with modern methods for data analysis.

It is well known that even under ideal conditions there is considerable error associated with the calculation of equilibrium constants (113-118). To illustrate, we contrast the porphyrin/viologen case with the simplest case involving two hypothetical absorbing species $A \& B$, each having no self-interactions. Further criteria should include: well resolved spectra of each monomer and the A-B association complex (minimal spectral overlap), known absorptivities for each, and 1008 conversion observed from $A$ to $A-B$ during the titration of $A$ with $B$ : $A+B \rightarrow A-B$ (a clear region where further addition of $B$ induces no further increase in the $A-B$ absorbance). In addition, the conversion from $A$ to $A-B$ should involve the formation of a strong association 
complex. This "well behaved" case is readily solved by fitting the loss in absorbance of $A$ or the increase in absorbance in A-B by linear regression (as a function of increasing B).

$\mathrm{H}_{2} \mathrm{TCPP}^{4-} / \mathrm{viologen}$ association is neither simple nor "well behaved" for the following reasons:

1) We have a multicomponent rather than a simple two-component equilibrium problem (by analogy to the hypothetical equilibrium described above, we have the formation of both $A-B$ and $B-A-B$ ). Molecular models show that doubly complexed porphyrins are reasonable, as two viologens could easily be accommodated by one face of a single $\mathrm{H}_{2}$ TCPP $^{4-}$ molecule (one across each side of the planar macrocycle). clear evidence for the formation of singly and doubly complexed porphyrin was the observation of two viologen concentration regions containing isosbestic points. The titration with $\mathrm{PVS}^{0}$ included, in addition to complexes with stoichiometries of $1: 1$ and 1:2, the formation of a porphyrin dimer (Figure 23B).

2) The spectra of each of the two viologen-associated $\mathrm{H}_{2} \mathrm{TCPP}^{4-}$ complexes were shifted by only a few nanometers to the red of the monomer porphyrin spectrum (Figure 23); therefore, the three components were not spectroscopically well resolved.

3) The association between porphyrins and viologens is weak. Association constants are most accurate when the equilibrium concentration is equal to the lowest concentration species $(116,117,119)$. In this case, the equilibrium concentration of the $1: 1$ association complex is approximately 3 orders of magnitude greater than the concentration of the porphyrin and the equilibrium concentration of the 1:2 species is approximately 5 orders of magnitude greater than the porphyrin concentration.

4) Porphyrins are not "well-behaved". Consider the equilibrium:

$$
\mathrm{H}_{2} \mathrm{TCPP}^{4-}+\mathrm{H}_{2} \mathrm{TCPP}^{4-} \Rightarrow\left(\mathrm{H}_{2} \mathrm{TCPP}^{4-}-\mathrm{H}_{2} \mathrm{TCPP}^{4-}\right)^{8-}
$$

Coulombic repulsion should make the forward reaction highly 
improbable; however, $\mathrm{H}_{2} \mathrm{TCPP}^{4-}$ appears to dimerize at $\mu \mathrm{M}$ concentrations as judged by the loss of linearity in the Beer's law plots (Figures 34 and 35 ). If $\mathrm{H}_{2} \mathrm{TCPP}^{4-}$ were a "classical" polyanion, then increased ionic strength should push the equilibrium to the left; the opposite effect is observed. This very limited solubility in aqueous solution is due, most probably, to the hydrophobic character of the porphyrin core.

If the driving force responsible for association can be ascribed to other than electrostatic factors, then the effect is usually explained as "entropy-driven" and considers the Gibbs free energy of the entire system, i.e. solute(s) plus solvent. Mixed entropic /electrostatic processes are more difficult to predict (i.e. equilibrium shifts in response to perturbations such as variations in solvent, temperature and ionic strength) and are probably responsible for many of our unexpected observations, such as the organic molecule-induced $\mathrm{H}_{2} \mathrm{TCPP}^{4-}$ dimerization and dimer dissociation illustrated in chapter IV. Calculating equilibrium constants for multicomponent, weakly complexing, spectroscopically unresolved systems is a challenge. The calculations are possible by nonlinear least-squares analysis (NLLS) if the set of associated equilibrium equations is known, if the error is minimal and if there are sufficient numbers of degrees of freedom. In any case, the solutions contain greater uncertainty than for a simple well-behaved case.

Strategy for calculating equilibrium constants

Considering the rather formidable problems outlined in the previous section, extensive studies were necessary in order to quantify the $\mathrm{H}_{2} \mathrm{TCPP}^{4-} / \mathrm{MV}^{2+}$ association constants and complex stoichiometry. In order to solve this multicomponent equilibrium problem we used the following strategy:

a) The degrees of freedom were maximized by taking an unusually large number of data points (we obtained between 30 and 120 points per 
titration).

b) The precision within each data set was optimized by using a titration method rather than by the method of individually prepared solutions.

c) The accuracy was checked by 1) repetition of each experiment 2) data analysis using two independent methods: nonlinear least-squares analysis and principal component analysis.

d) The number of variables in the NLLS analysis was minimized by reducing the number of absorbing solution species. This was accomplished by choosing experimental conditions where only monomer porphyrin is in solution thereby eliminating the porphyrin monomer/dimer equilibrium equation. In addition, the absorptivity of the highest complex formed (1:2) was determined directly from the spectral data. This was accomplished by titrating to sufficient viologen concentrations where further incremental viologen addition induced no change in the spectra. These criteria indicate complete conversion to the highest complex formed.

\section{SPECTROPHOTOMETRIC TITRATION OF $\mathrm{H}_{2} \mathrm{TCPP}^{4-}$ WITH $\mathrm{MV}^{2+}$}

In this section data from four titration experiments are presented. Each spectrophotometric titration of $1.00 \mu \mathrm{M} \mathrm{H}_{2} \mathrm{TCPP}^{4-}$ in aqueous solutions $\left(\mathrm{pH}=7.0,20^{\circ} \mathrm{C}\right)$ at either constant buffer concentration or constant ionic strength included 30 to 120 incremental viologen concentrations.

\section{Methods and Results of MLIS Analyses, Constant Buffer Concentration}

In the titration at constant buffer concentration, the only variable is the viologen concentration (neglecting the inadvertent alkali metal cation concentration variability); this technique, where the titrant contains porphyrin $(1.00 \mu \mathrm{M})$ and buffer $(5.00 \mathrm{mM}$ $\left.\mathrm{KH}_{2} \mathrm{PO}_{4} / \mathrm{NaKHPO}_{4}\right)$ concentrations identical to the solution in the cuvette, was defined in Table $\mathrm{V}$, method $2 \mathrm{C}$. Therefore, the spectral changes are 
only a function of increments in viologen concentration.

Experiment 1. In the first experiment the effect of both very low $(.10 \mu \mathrm{M})$ and intermediate to high (10 $\mathrm{mM})$ viologen concentrations are studied by spectrophotometric titration. Because of the large number of points taken, the data were collected over two days using dilutions of the same viologen stock solution. Data between 385 and $450 \mathrm{~nm}$ were collected.

This experiment was performed prior to installation of the computer interface, thus we were limited by the capacity of the instrument to store and give hard copy outputs of the absorbance at 6 wavelengths. For each incremental viologen addition, data at 414.1 and $414.2 \mathrm{~nm}$ were averaged and analyzed by nonlinear least-squares (NLLS) as described in Chapter III. The raw absorbance data and the best fit curve from the NLLS analysis are shown in Figure 41 . Notice that the two curves do not overlap precisely even though these experiments were performed using the same stock solutions. This variability was present in comparing all our titration data sets and was similar to that discussed in Chapter IV where, by a process of elimination, we attributed the lower than expected accuracy between Beer's law experiments to the concentration of alkali metal ions released from the glassware. In this set of experiments two different cuvettes (glass, pathlength $=2.08$ and quartz, pathlength $=2.2$ ) were used. It is logical that they may differ either in the number of anionic sites or by inadvertent unequal loading of the sites with alkali metal cations during the washing procedure.

We obtained a hard copy overlay of the absorbance and difference spectra at each viologen concentration. The isosbestic points were obscured by the large number of incremental concentration points with their respective spectra; therefore, an analogous experiment was performed using viologen concentrations which best illustrate these regions (Figure 44). For notational convenience we will refer to these 
three data sets as "experiment 1 ".

Experiment 2. In an additional titration (Figure 42) we investigated the high viologen concentration region and confirmed that there exists a limiting viologen concentration where additional viologen

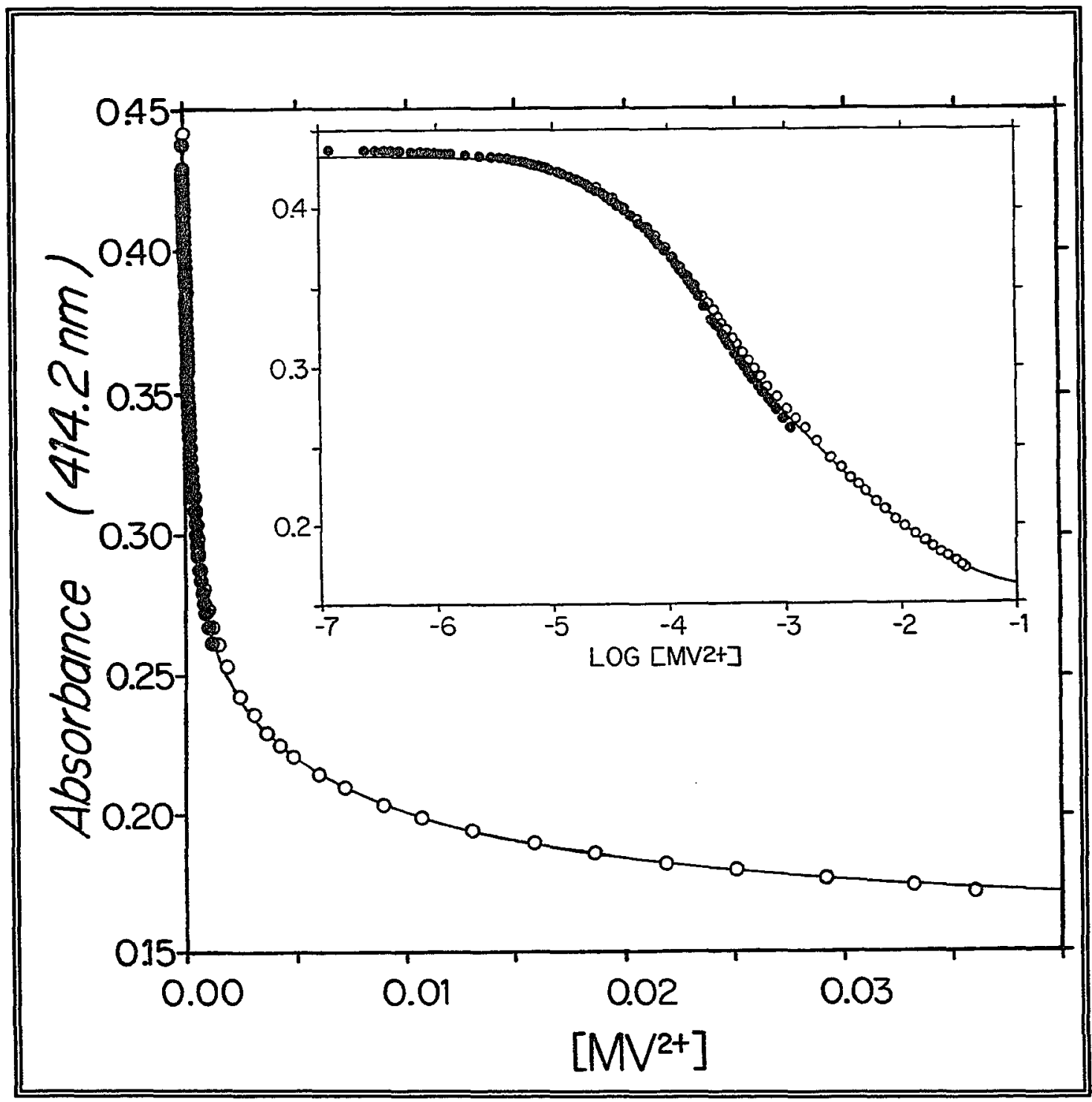

Figure 41. Spectrophotometric titration of $\mathrm{H}_{2} \mathrm{TCPP}^{4-}$ with $\mathrm{MV}^{27}$, experiment 1. Each point represents the porphyrin absorbance measured at $414.2 \mathrm{~nm}$ for one incremental viologen addition ( $\left[\mathrm{MV}^{2+}\right]$ is Molar): 0 shows the study at low and 0 at intermediate to high viologen concentrations, respectively. The data are plotted on both linear and semilog (inset) scale. The line is the nonlinear least-squares fit. 

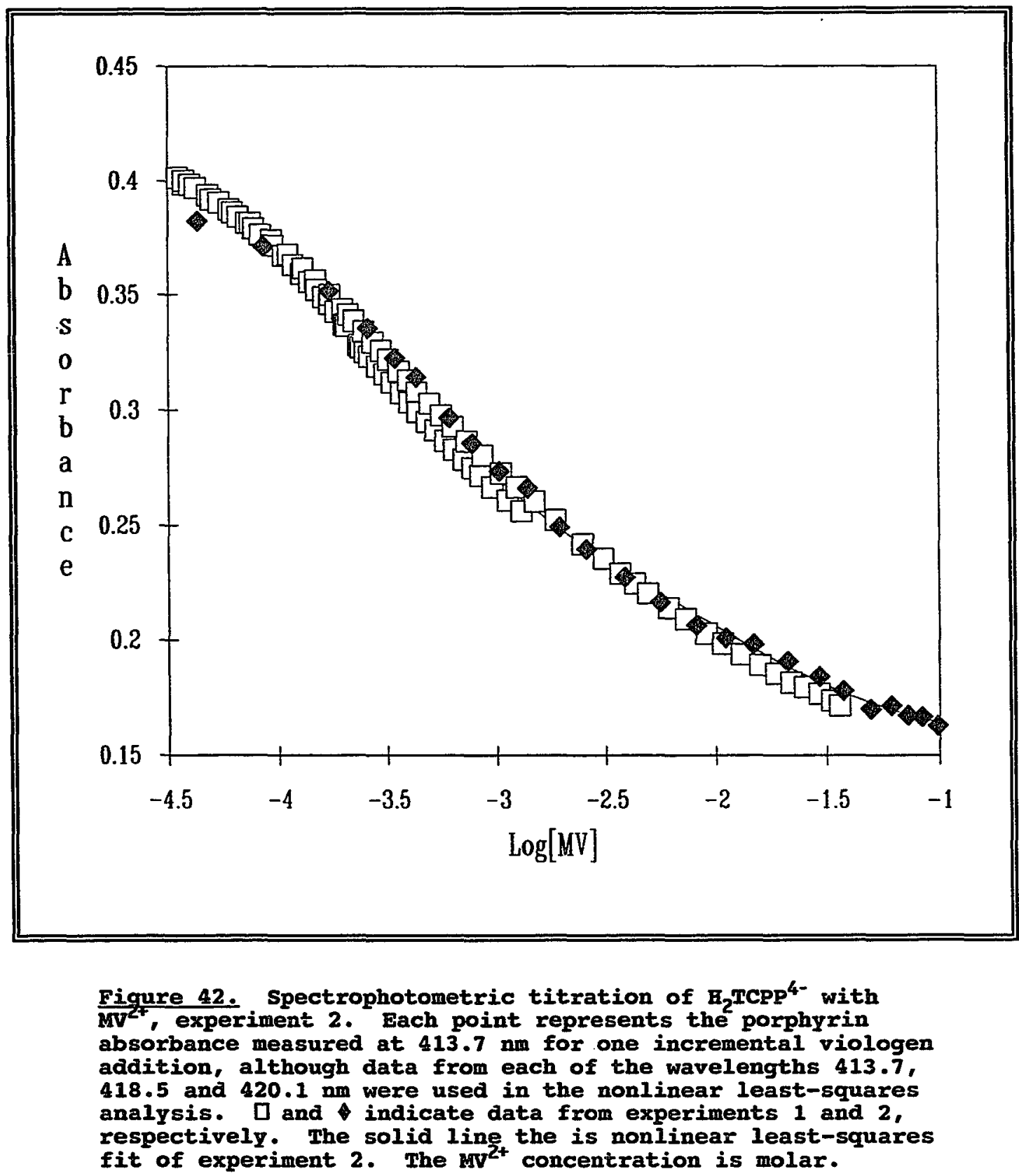

caused no further drop in the absorbance. We also confirmed that no net viologen was being reduced by collecting absorbance data from 380 to 700 $\mathrm{nm}$. We observed no increase in the absorbance at $602 \mathrm{~nm}$, an absorbance maximum of the reduced viologen. This spectrophotometric titration was performed after the spectrophotometer was interfaced with the 
microcomputer which allowed us to select representative concentration points and wavelength regions to illustrate patterns. Selected spectra from this titration were shown in Figure $24 \mathrm{~A}$.

since the spectral data were stored on the microcomputer (we acquired 3,200 absorbance data points per viologen concentration increment), we were also able to analyze this data set by both NLIS (at three wavelengths) and PCA. Figure 42 shows the raw titration absorbance data at $413.7 \mathrm{~nm}$ and the best fit line (partially obscured by data points) from the NLIS analysis versus the $\mathrm{MV}^{2+}$ concentration. For the purpose of comparison the data from experiment 1 are included, notice that the curves overlay satisfactorly (with the exception of the point at lowest $\left[\mathrm{MV}^{2+}\right]$ taken].

The treatment of the data by PCA is detailed in a later section of this chapter. For notational convenience we refer to this data set as "experiment $2 "$.

\section{Methods and Results of MLIS Analysis, Constant Ionic Strength}

In Chapter IV we discussed in detail the problems associated with increased alkali metal ion concentration; earlier in this chapter the uncertainties associated with the analysis of data containing two variables with competing effects were mentioned. Increased alkali metal ion concentation induces a spectral line shape change at concentrations below $40 \mathrm{mM}$ and at higher concentrations there are blue shifts in addition to changes in the line shape. Viologens induce concentration dependent red-shifts and weakening. In experiments performed at constant ionic strength, the alkali metal ion concentration must decrease as the viologen concentration increases in order to fix the ionic strength at a constant value: hence, a simultaneous study of the effect of two variables. Each variable effects the parameter being measured, the absorbance.

Experiment 3. In the experiment illustrated in Figure 43, we used method 2D described in Chapter III, Table $V_{;}$the experimental conditions 


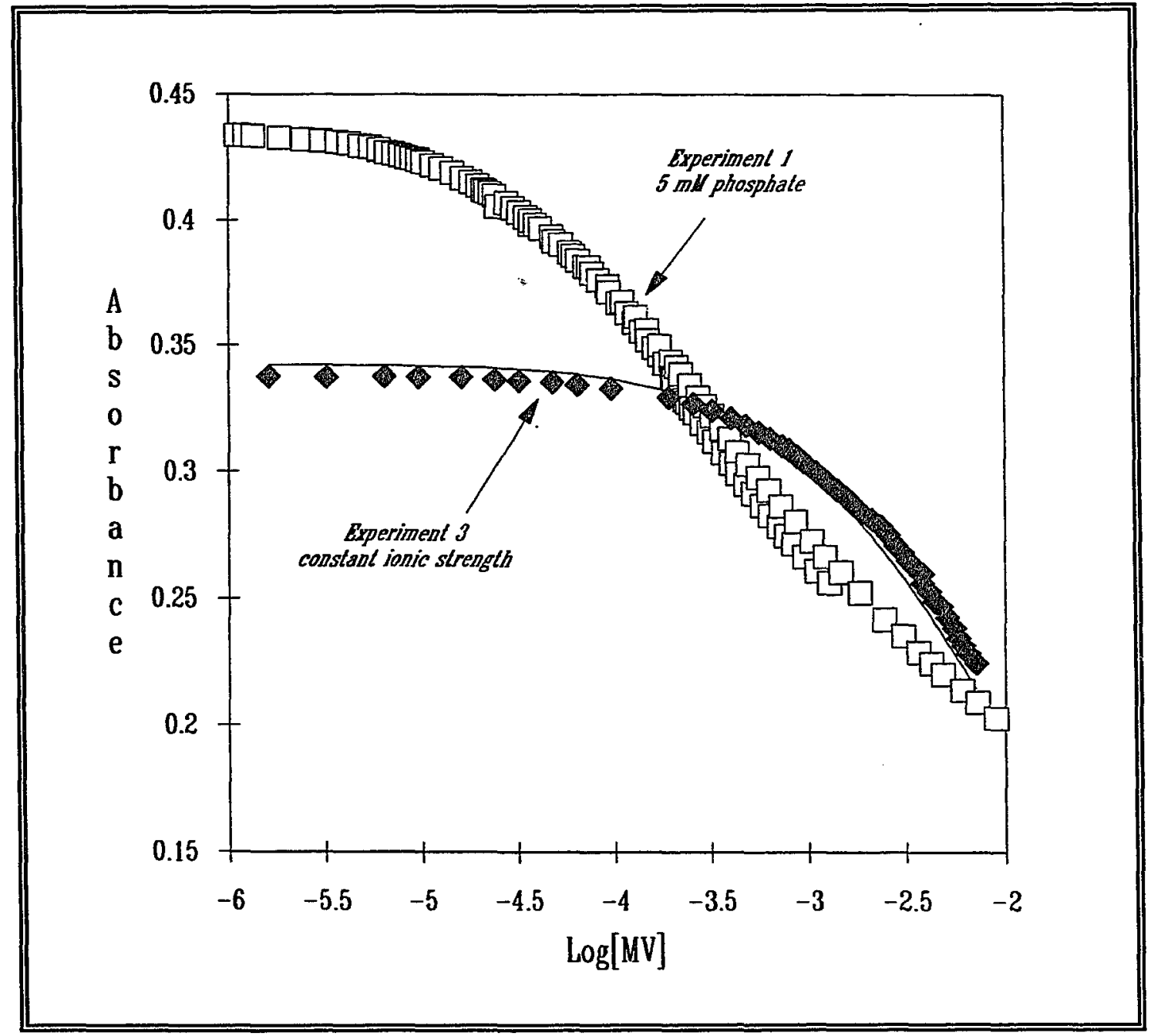

Figure 43. Spectrophotometric titration of H,TCPP $_{2}^{4-}$ with MV ${ }^{2 t}$, experiment 3 . Each point represents the porphyrin absorbance measured at $413.6 \mathrm{~nm}$ for one incremental viologen addition, although data from each of the wavelengths 413.6 , 416.2 and $419.8 \mathrm{~nm}$ were used in the nonlinear least-squares analysis. $\square$ and indicate data from experiments 1 and 3 , respectively. The solid line is least-squares fit of experiment 3 . The viologen concentration is molar.

were $\mathrm{pH}=7.0$ and $\mathrm{T}=20^{\circ} \mathrm{C}$. In experiment 3 the ionic strength was maintained at 0.15 with $\mathrm{KH}_{2} \mathrm{PO}_{4} / \mathrm{NaKHPO}_{4} ;$ the ionic strength was 1.5 maintained with $\mathrm{KCl}$ in experiment 4 , where the phosphate concentration was held constant (at $5.00 \mathrm{mM}$ ) by the previously described technique of adjusting the titrant and solution in the cuvette to identical 
concentrations. As in all the titration experiments, the porphyrin concentration was $1.00 \mu \mathrm{M}$. Figure 36 illustrated the spectral change induced by $62 \mathrm{mM}$ phosphate, which was the initial phosphate concentration used to obtain an ionic strength of 0.15 . This effect can also be observed in Figure 43, which compares the viologen concentration dependent porphyrin absorbance at 414.2 (experiment $1,5.00 \mathrm{mM}$ phosphate) with the porphyrin absorbance at 413.6 (experiment 3 , initial phosphate concentration $=62 \mathrm{mM}$ ).

As previously discussed, we interpreted this phosphate-induced change in the soret region of $\mathrm{H}_{2} \mathrm{TCPP}^{4-}$ as a spectral line shape change, which was contrary to the accepted explanation. Other authors accept the model of salt-induced porphyrin dimerization. In light of our controversial data interpretation, and the uncertainties associated with correlating line shape changes with solution phenomena, we were prompted to analyze the data via a method which was "model independent". The use of PCA to study these data was suggested by Dr. Martin Gouterman; the details of the analysis are extensively discussed in a later section. The results of the analysis imply that the dimer equilibrium should not be included.

For this set of data, the NLLS analysis always yielded at least one unreasonable parameter (the optimization was performed at least 10 times), this was usually an unreasonably low absorptivity value for the 1:2 porphyrin:viologen complex. This was most likely due to the fact that insufficiently high viologen concentrations were used to obtain complete conversion to the doubly complexed species. The highest viologen concentration used was limited by the ionic strength chosen.

Experiment 4. In experiment 4 we increased the ionic strength in order to be able to use high enough viologen concentration to obtain complete conversion, but were not successful. It appears that increased ionic strength shifts the equilibria to higher viologen concentrations; although there may be an optimal ionic strength within which there 
exists a viologen concentration region where complete conversion to the doubly complexed species can be observed, we were unable to find such conditions.

The spectral data observed as a function of increased viologen concentration differed from that observed in experiments 1-3. Since isosbestic points were absent, we were unable to analyze the data by NLLS analysis or PCA.

This experiment was performed three times with the same result, twice with $\mathrm{H}_{2} \mathrm{TCPP}^{4-}$ and once with $\mathrm{H}_{2} \mathrm{TSPP}^{4-}$. The spectral patterns are shown in Figure 46.

Evidence for $\mathrm{H}_{2} \mathrm{TCPP}^{4-}: \mathrm{NV}^{2+}$ complexes With Stoichiometries of $1: 1$ and $1: 2$

The spectral data from experiment 1 shown in Figure 44 illustrate the presence of two clear isosbestic points separated by a viologen concentration region where no isosbestic region exists. We use this experiment to illustrate the spectral patterns observed in each of the experiments 1-3. Following this discussion we compare the patterns observed in experiments 1-3 with that observed in experiment 4.

Each titration shows five distinct regions labeled a-e in Figure 44; our interpretations are detailed in the set of equations in Table XI which define the "model" used in the NLLS analysis. In Table XII we correlate each equation in the model with specific $\mathrm{MV}^{2+}$ concentration ranges.

In Table XI, "....." in equations 1 and 4 , implies only that the addition of $\mathrm{MV}^{2+}$ results in spectral changes inconsistent with simple complexation; therefore, the phenomena responsible for the spectral change described in Table 2, region "a" may be due to "wall effects" (equation 1 ) and region " $e$ " to "mixed-solvent" effects (equation 4). The addition of $\mathrm{MV}^{2+}$ represents the increasing concentration of an organic molecule, and may affect the porphyrin spectrum in a manner analogous with all organic molecules. As was described in chapter IV, the addition of organic salts or solvents results in a red-shifted, but 
not necessarily weakened, spectrum.

Two species connected by "-", represent association in the conventional sense, thus equations 2 and 3 represent equilibria.

TABLE XI

MODEL USED IN THE NLLS ANALYSIS

\begin{tabular}{|c|c|}
\hline EQUATION & EQUILIBRIUM EQUATIONS (MODEI) \\
\hline \hline 1 & $\mathrm{H}_{2} \mathrm{TCPP}^{4-}+\mathrm{MV}^{2+}-\mathrm{H}_{2} \mathrm{TCPP}^{4-} \ldots . . . \mathrm{MV}^{2+}$ \\
\hline 2 & $\mathrm{H}_{2} \mathrm{TCPP}^{4-}+\mathrm{MV}^{2+}-\left(\mathrm{H}_{2} \mathrm{TCPP}^{4-}-\mathrm{MV}^{2+}\right)^{2-}$ \\
\hline 3 & $\left(\mathrm{H}_{2} \mathrm{TCPP}^{4-}-\mathrm{MV}^{2+}\right)^{2-}+\mathrm{MV}^{2+}-\left(\mathrm{MV}^{2+}-\mathrm{H}_{2} \mathrm{TCPP}^{4-}-\mathrm{MV}^{2+}\right)^{0}$ \\
\hline 4 & $\left(\mathrm{MV}^{2+}-\mathrm{H}_{2} \mathrm{TCPP}^{4-}-\mathrm{MV}^{2+}\right)^{0}+\mathrm{MV}^{2+}-\left(\mathrm{MV}^{2+}-\mathrm{H}_{2} \mathrm{TCPP}^{4-}-\mathrm{MV}^{2+}\right)^{0} \ldots \ldots \cdot \mathrm{MV}^{2+}$ \\
\hline
\end{tabular}

Table XII

DATA SUMAARY: TITRATION OF $\mathrm{H}_{2} \mathrm{TCPP}^{4-}$ WITH $\mathrm{Mv}^{2+}$

\begin{tabular}{|c|c|c|c|c|c|c|c|c|c|c|}
\hline \multirow[t]{2}{*}{ Region } & \multirow{2}{*}{$\begin{array}{l}\text { Equation } \\
\text { Table } x\end{array}$} & \multicolumn{3}{|c|}{ Isosbestic $\lambda$} & \multicolumn{3}{|c|}{$\left[M V^{2+}\right]$ Range } & \multicolumn{3}{|c|}{ Equilibrium Constant } \\
\hline & & 1 & 12 & 3 & If $1(\mathrm{~m} / \mathrm{H})$ & N 2 (m) & $3(\mathrm{mM})$ & 1 & 12 & 3 \\
\hline $\mathbf{a}$ & 1 & none & none & none & $.1 \mu \mathrm{M} \rightarrow .043$ & $<0.043$ & $.0016-.049$ & $=80,000$ & not done & not done \\
\hline b & 2 & 418.6 & 418.0 & 417.5 & $.043 \rightarrow 0.34$ & $.043 \rightarrow 0.35$ & $0.048 \rightarrow 1.28$ & $3171 \pm 47$ & 3350 & 594 \\
\hline c & $2 \& 3$ & none & none & none & $0.34 \rightarrow 14.0$ & $0.35 \rightarrow 14.9$ & $1.28+5.16$ & N.A. & N.A. & H.A. \\
\hline d & 3 & 421.4 & 420.7 & 420.7 & $14.0 \rightarrow 85.0$ & $14.9-97.9$ & $5.16 \rightarrow 7.17$ & $108 \pm 5$ & 68 & 38 \\
\hline e & 4 & none & none & none & $>85.0$ & $>97.9$ & not done & N.A. & N.A. & N.A. \\
\hline
\end{tabular}

Where $W^{2}=$ Experiment 1,2 , or 3 as described in the text; N.A. means not applicable. 


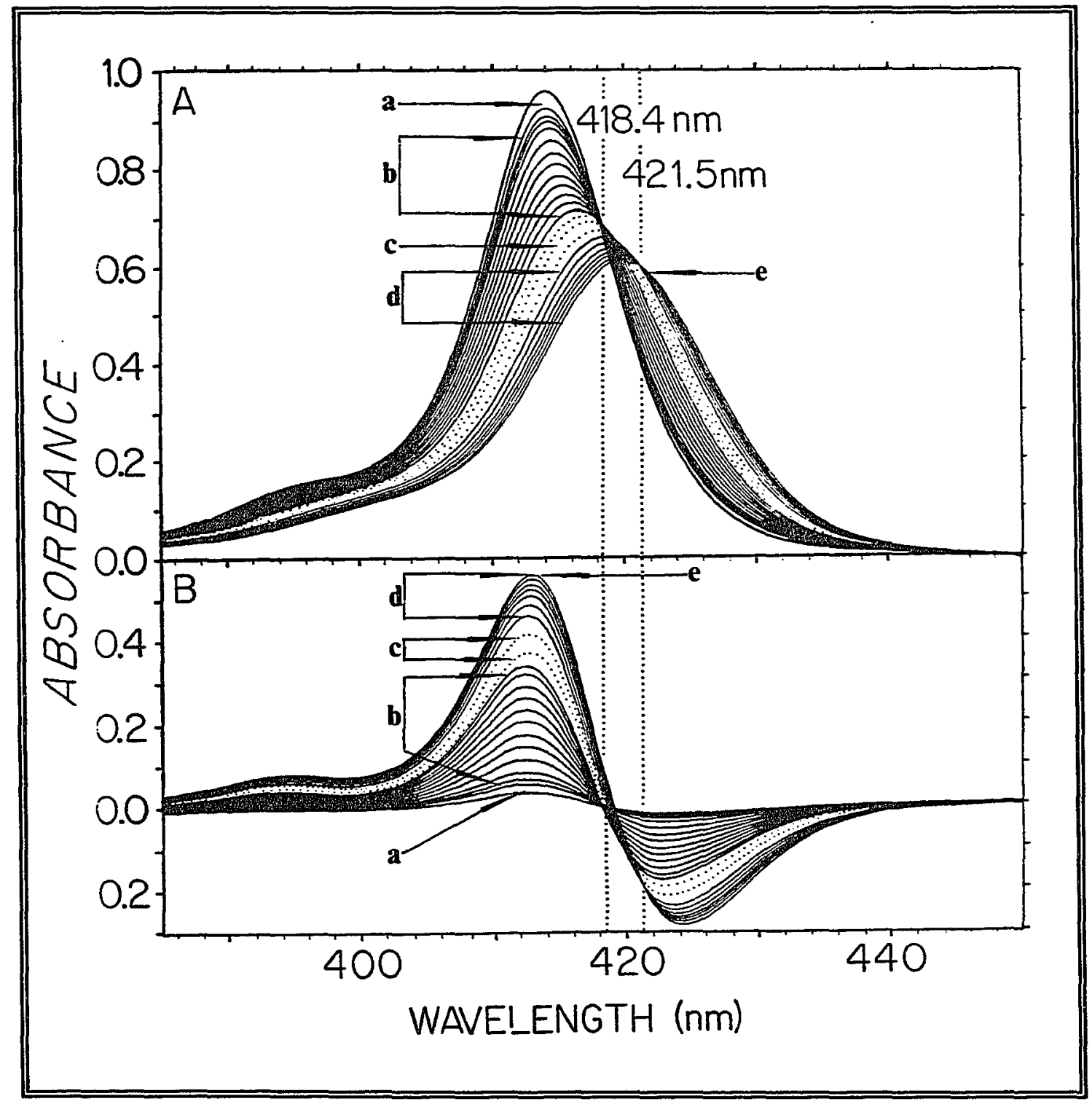

Figure 44. Titration of $\mathrm{H}_{2} \mathrm{TCPP}^{4-}$ with $\mathrm{MV}^{2+}$, experiment 1 . A) Selected spectral data Irom the titration. B) Difference spectra obtained by subtracting each spectrum in the titration from the spectrum of monomer porphyrin (the initial spectrum). The regions in the spectra labeled "a""e" correspond to Tables XI, XII and the discussion in the text. The spectra obtained at viologen concentrations where no isosbestic point was observed are indicated by "....."

Region a) In each titration there was an initial viologen concentration region where no isosbestic point was observed; it was particularly noticeable when the low concentration region was studied 
(as in experiment 1), otherwise it was observed as the failure of the spectrum of the porphyrin only (no viologen) to pass through the isosbestic point (experiment 2).

The viologen concentration dependence of this region varies from one experiment to another and is probably related to the number of anionic sites on the surface of the cuvette, or more specifically, to the number of alkali metal cations which were loaded onto these sites. We do not think that this initial anomaly is due to dissociation of porphyrin dimers since porphyrin dimers should not be present under these conditions (Beer's law data, Chapter IV).

Region b) At some threshold viologen concentration, a concentration range containing isosbestic points began, indicating the highest viologen concentration where "wall effects" were observed, leaving monomer and its $1: 1$ complex as the only species in solution. Compared to monomer $\mathrm{H}_{2} \mathrm{TCPP}^{4-}$, the $1: 1$ porphyrin:viologen complex had a lower absorptivity and a red-shifted $\lambda_{\max }$. This explains the spectral changes observed as a function of increased viologen concentration: the concentration of $\mathrm{H}_{2} \mathrm{TCPP}^{4-}$ decreased and the concentration of $\mathrm{H}_{2} \mathrm{TCPP}^{4-}-\mathrm{MV}^{2+}$ (1:1 complex) increased.

Region c) The first isosbestic point was lost at the lowest viologen concentration where the presence of the electrically neutral $\mathrm{MV}^{2+}-\mathrm{H}_{2} \mathrm{TCPP}^{4-}-\mathrm{MV}^{2+}(1: 2)$ complex was observed. Since three absorbing species were present throughout this region, (porphyrin monomer, 1:1 and 1:2 complexes) no isosbestic was observed. The porphyrin monomer was detected to very low concentrations due to its high absorptivity.

Region d) At very high viologen concentrations a second region containing isosbestic points was observed; its first appearance marked the viologen concentration where all porphyrin was present as either the 1:1 or 1:2 complex. The spectral changes resulted from the conversion of $\left(\mathrm{MV}^{2+}-\mathrm{H}_{2} \mathrm{TCPP}^{4-}\right)^{2-}$ to $\left(\mathrm{MV}^{2+}-\mathrm{H}_{2} \mathrm{TCPP}^{4-}-\mathrm{MV}^{2+}\right)^{0}$ which has, relative to the $1: 1$ complex, a lower absorptivity and red-shifted absorbance maximum. 
Region e) A point was reached in the titration which indicated complete conversion to the $1: 2$ species. What was observed spectroscopically is that further increase in the viologen concentration caused no significant spectral change (a slight red-shift was observed). Increasing the temperature (from $20^{\circ} \mathrm{C}$ to $50^{\circ} \mathrm{C}$ ), at viologen concentrations well above this point, caused a slight red shift $1 \approx 0.5$ nm) which may indicate some change in the organization of the complex.

The set of spectra obtained for experiment 2 resemble those of experiment 1. These spectra were used in the PCA analysis and are shown in Figure 48 in the following section.

With the exception of the weakened Soret band, the spectra obtained in experiment 3 also resemble experiments 1 and 2 . Selected spectra from this titration along with the associated difference spectra are presented in Figure 45. The labels a-e on the graph refer to the spectral regions and associated equilibria described in Tables XI and XII and are exactly analogous to Figure 44 .

Figure 46 shows the unusual spectral patterns observed in the titration of $\mathrm{H}_{2} \mathrm{TCPP}^{4-}$ with $\mathrm{MV}^{2+}$ at high constant ionic strength. The experimental data set contained 80 spectra; representative spectra were chosen from this set to illustrate the unusual trends which resemble those observed by other authors $(46,51,57)$ who interpret the increase in absorptivity at $\approx 422 \mathrm{~nm}$ as the appearance of the 1:1 porphyrin:viologen complex. We question this interpretation as we find that an increase in the ionic strength results in a blue-shifted spectra for the $1: 1$ and $1: 2$ porphyrin/viologen complexes (Figure 55). The 1:1 complex has a $\lambda_{\max }$ at approximately $417 \mathrm{~nm}$.

It appears that $1.5 \mathrm{M} \mathrm{KCl}$ induces porphyrin dimerization as the spectral patterns resemble, in their complexity, organic moleculeinduced porphyrin dimer dissociation, analogous to the dissociation of $\mathrm{H}_{2}$ TSPP $^{4-}$ dimers by crown ether illustrated in Figure 28. One plausible interpretation considered that at this high alkali metal ion 


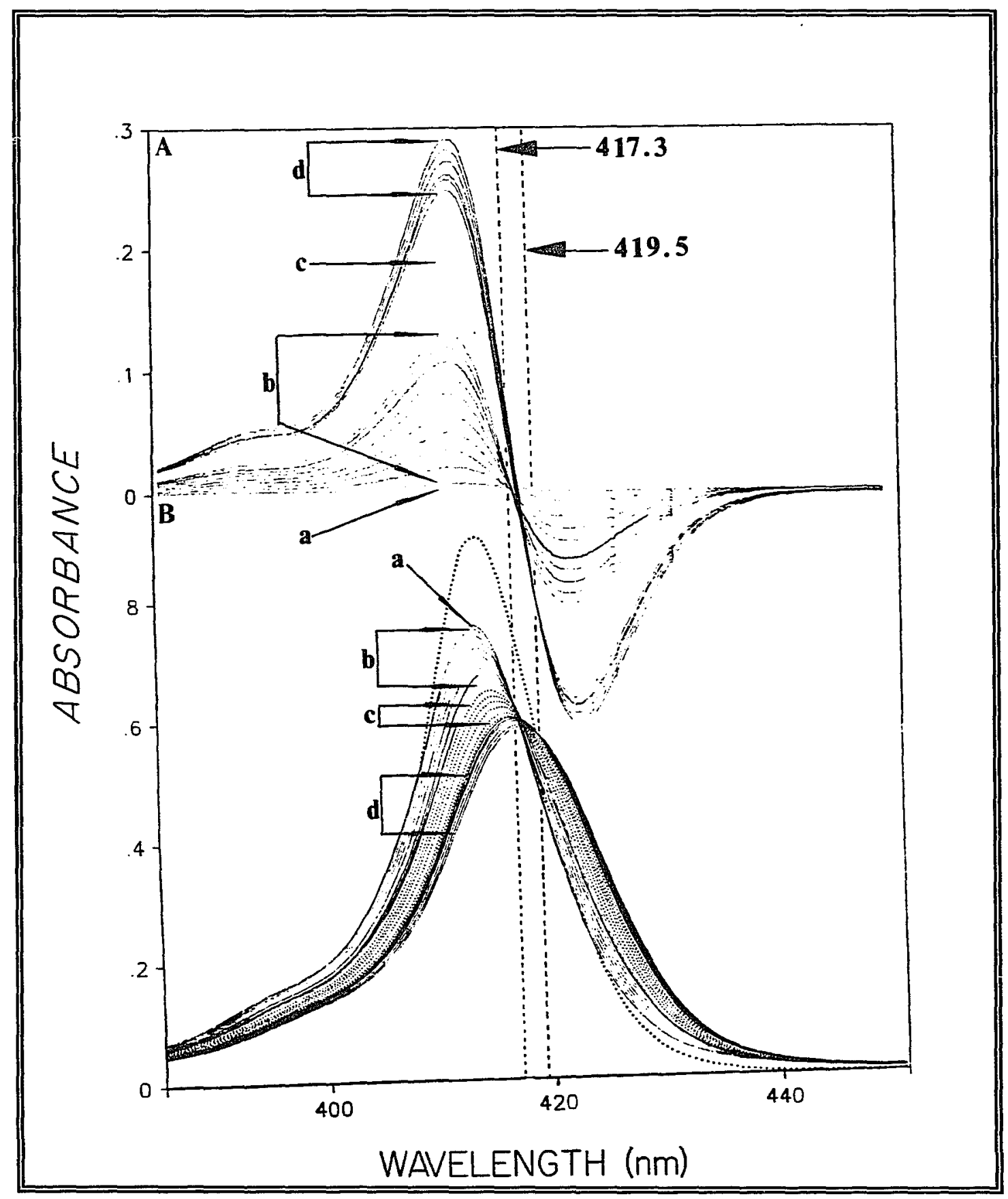

Ficure 45. Titration of H TCPP $^{4-}$ with $\mathrm{Mv}^{2+}$ experiment 3; Spectra and difference spectra. "..." indicates low ionic strength, "...." indicates spectra between isosbestic regions. A) Difference spectra (spectrum of uncomplexed porphyrinobserved spectrum); onlY spectra which pass through an isosbestic point are shown. B) Selected spectral data from the titration. The regions in the spectra labeled "a"-"e" correspond to Tables XI, XII and text. 
concentration porphyrin dimers are in equilibrium with monomers; evidence for this was the broadened and blue-shifted initial spectra. The progressive red shift and weakening of the soret $\lambda_{\max }$ as a function of incremental viologen addition could be attributed to the formation of porphyrin:viologen association complexes. The absorbance minimum may be the lowest viologen concentration before dimer dissociation is induced; higher viologen concentration would induce further dimer dissociation, hence the sharpened spectrum and the increase in absorbance at the soret $\lambda_{\max } \cdot$

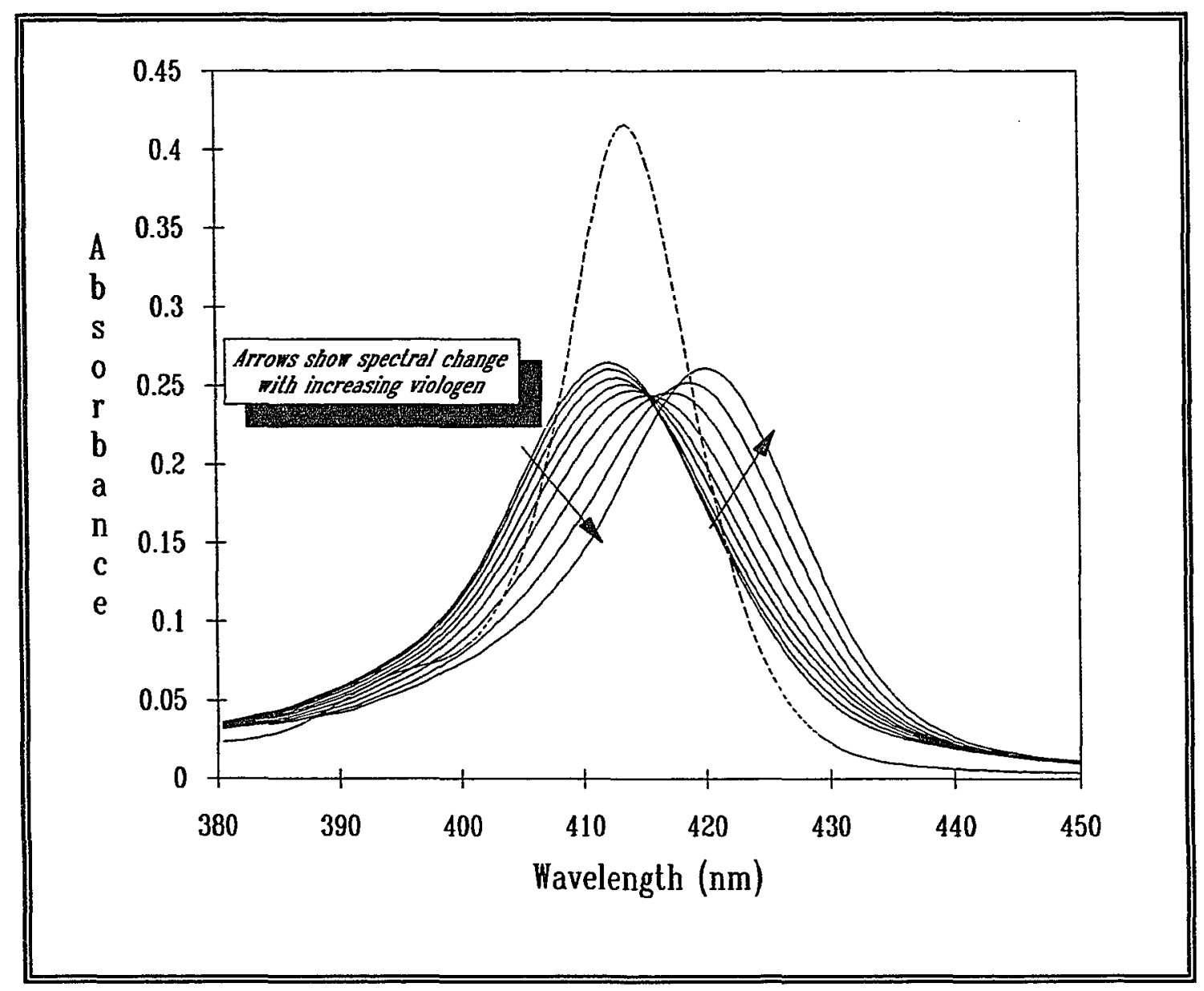

Figure 46. Titration of $\mathrm{H}_{3} \mathrm{TCPP}^{4-}$ with $\mathrm{MV}^{2+}$; experiment 4." "-" indicates $5.00 \mathrm{mM}$ phosphate at $\mathrm{pH}=7.0$. Each spectrum illustrated as a solid line indicates an incremental increase in the viologen concentration at $I=1.5$ maintained with $\mathrm{KCl}$ and $\mathrm{MV}^{2+},\left[\mathrm{KH}_{2} \mathrm{PO}_{4} / \mathrm{NaKHPO}_{4}\right]=5.00 \mathrm{mM}, \mathrm{T}=20^{\circ} \mathrm{C}$. 
Methods and Analysis by PCA, Constant Buffer Concentration

Experiment 2. As was mentioned earlier, PCA gives information concerning the number of absorbing species in solution $i . e$. is "modal independent". However, it does assume Beer's law, meaning that the resultant "observed spectra" must be a linear combination of the spectral line shapes of the components.

The flow diagram in Figure 23 outlined the PCA process which we illustrate step-by-step for experiment 2 below. The first step was to determine the number of unique absorbing species in the data set which, after eliminating certain spectra due to monochrometer slip error, comprised 25 spectra each corresponding to a different viologen concentration. In each spectrum, which contained 3200 absorbance measurements in the wavelength range $385-700 \mathrm{~nm}$, we selected 200 absorbance data points equally spaced along the wavelength scale. A 200 x 25 matrix was constructed from the data and multiplied by its transpose to give the 200 x 200 data covariance matrix. The eigenvectors and eigenvalues were calculated from the data covariance matrix using a published FORTRAN program (138). The subroutines were adapted and the mathematical analysis was performed by Harry Bell. In this first part of the process we were interested in the eigenvalues, shown in Figure 47, which can be related to the number of principal components (104-112). We have explained our spectrophotometric titration data by two equilibrium equations ( 2 and 3 in Table XI) which means that we assume three principal components (absorbing species).

While there have been studies of methods of determining the "true" number of components in the presence of noise $(106,107,110,112)$, in practice it is usually sufficiently easy to distinguish the signal from noise by visual inspection. Each eigenvalue has the dimensions of absorbance variance and the data well below the instrumental noise level are clearly noise. Notice that three eigenvalues are above the "noise 


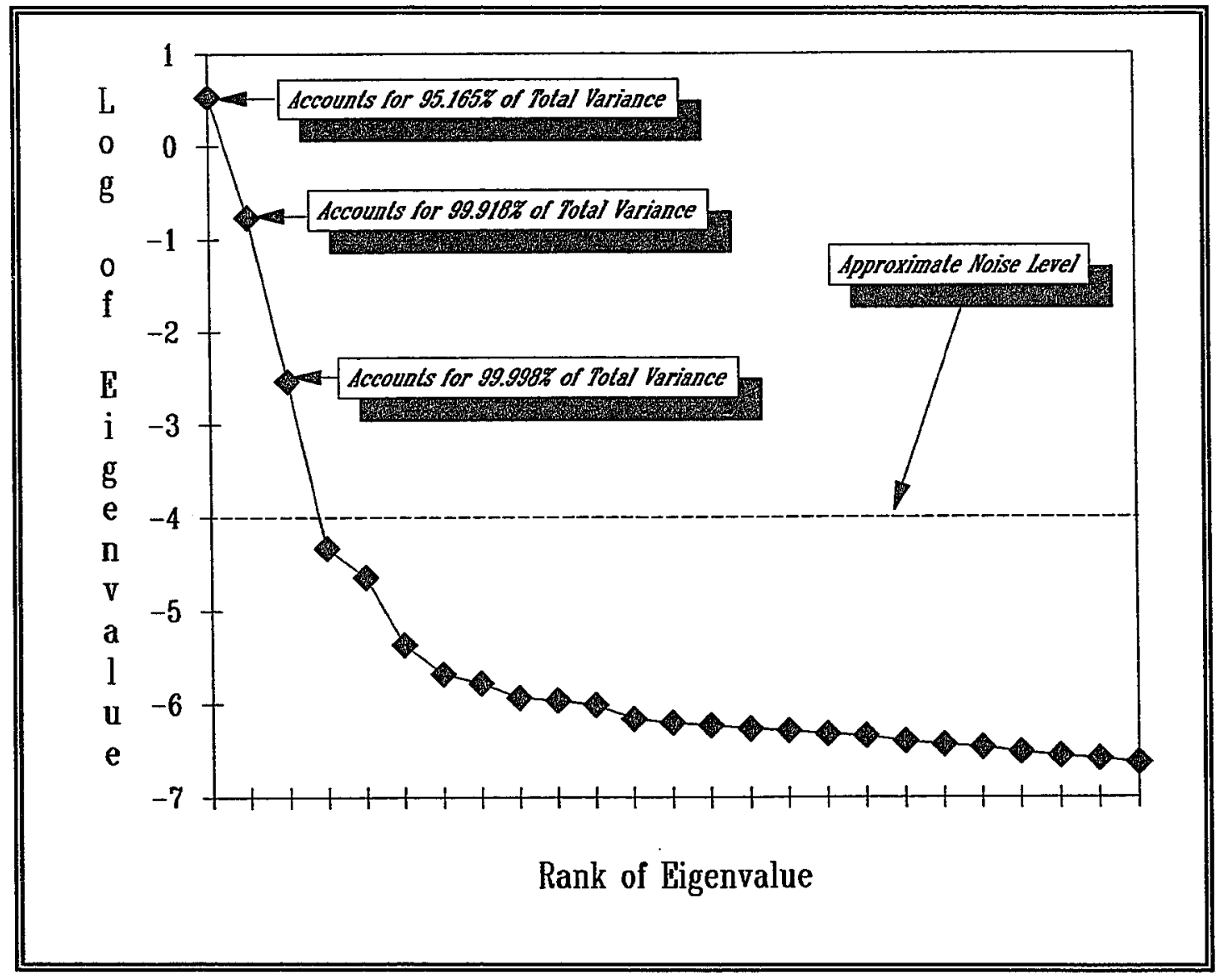

Figure 47. Three principal components in the spectral data from experiment 2. Results from the PCA analysis of 25 spectra, 200 absorbance data points/spectra. Absorbance data points at equally spaced wavelengths were selected in the wavelength region from 700 to $350 \mathrm{~nm}$. The "cumulative" variance was calculated as: (top), the $\{$ (largest

eigenvalue)/( $\Sigma$ eigenvalues) \}; (second), $\{$ ( $\Sigma$ two largest eigenvalues)/( $\Sigma$ eigenvalues) $\} ;($ third), $\{(\Sigma$ three largest eigenvalues $) /(\Sigma$ eigenvalues $)\}$.

level" in Figure 47 indicating three principal components which confirmed our model of two equilibrium equations and three unique absorbing species. A "cumulative" variance is shown in Figure 47. These values are interpreted as follows:

The largest eigenvalue: This means that if you assumed that there was only one component in solution you could account for 95.28 of the variance. 
The two largest eigenvalues: If you assumed that there were two components in solution you could account for 99.98 of the variance.

The three largest eigenvalues: If you assumed that there were three components in solution you could account for 1008 (99.998) of the variance.

If the spectra of each unique component were well resolved from the spectra of all other unique components, one would expect the variances associated with the largest eigenvalues to be well resolved and to unambiguously reflect the number of absorbing species in solution. However, we have just described a case which could be interpreted without using PCA. The three absorbing species $\mathrm{H}_{2} \mathrm{TCPP}^{4-}$, $\mathrm{H}_{2} \mathrm{TCPP}^{4-}-\mathrm{MV}^{2+}$, and $\mathrm{MV}^{2+}-\mathrm{H}_{2} \mathrm{TCPP}^{4-}-\mathrm{MV}^{2+}$ are not spectroscopically well resolved; therefore, we expect to observe eigenvalues that reflect the fact that the spectra of the three components are separated by only a few nanometers. If more spectra had been included in the analysis the noise level would have dropped, this would have eliminated the ambiguity of having two eigenvalues with values between $1 e-4$ and $1 e-5$ (see second PCA analysis, Figure 52). Under the experimental conditions of this specific example (low and constant buffer concentration) we know that no porphyrin dimer exists in solution; therefore, this is a "test" case in which we already know that there are three, and only three, species in solution.

In the analysis of spectroscopic data, the noise level is usually set between $1 e-4$ and $1 e-5$ depending upon the number of data points, the signal-to-noise ratio of the absorbance readings and the reproducibility of the wavelength. In this case the monochrometer drive was beginning to slip (a problem which became progressively worse), and only 25 spectra were used, which explains the relatively high noise level. In step 2 we derived the spectral line shape and the absorptivities for each of the three components. To the best of the author's knowledge this has not been done for a three-component system 
as it involves manipulations in three-dimensional space. In what we believe to be a novel method, developed by Harry Bell and the author, we were able to reduce the problem to two two-dimensional manipulations by using spectral data which passed through an isosbestic point. Figure 48 (top) shows the spectrophotometric titration data in experiment 2 which passed either through the first or second isosbestic point. This represents the data used in the two following analyses where the process illustrated in Figure 47 is repeated for the spectra passing through the first isosbestic point (Figure 48, bottom left) and for data passing through the second isosbestic point (Figure 48, bottom right). By this process we confirmed that the data within each viologen concentration region containing an isosbestic point is the linear combination of the absorptivities of only two absorbing species.

In step 3 we illustrate how the spectral line shapes and absorptivities are derived from the eigenvalues and eigenvectors by a "visual" optimization process.

Figure 49 (top) shows the preliminary results of the principal component analysis of experiment 2 using only those spectra at viologen concentration increments which pass through the first isosbestic point. The coordinates are the coefficients of the first two principal eigenvectors of the data covariance matrix. What must be determined are two points in this plane (two pair of coefficients). In this graph, any ray emanating from the origin defines a spectral line shape; each $x, y$ point along a ray defines both the line shape and the absorptivity. The upper and lower wedge regions are simply domains in which the resulting component spectral shapes are nonnegative at all wavelengths. One component must be located in the upper wedge region and the other component in the lower wedge region (defining the line shape and absorptivity of each component). The two data matrices ( $200 \times 5$ and 200 $x$ 9) were used to find the domains where all absorptivities are nonnegative (to locate the two wedge-shaped regions). Between the wedge 


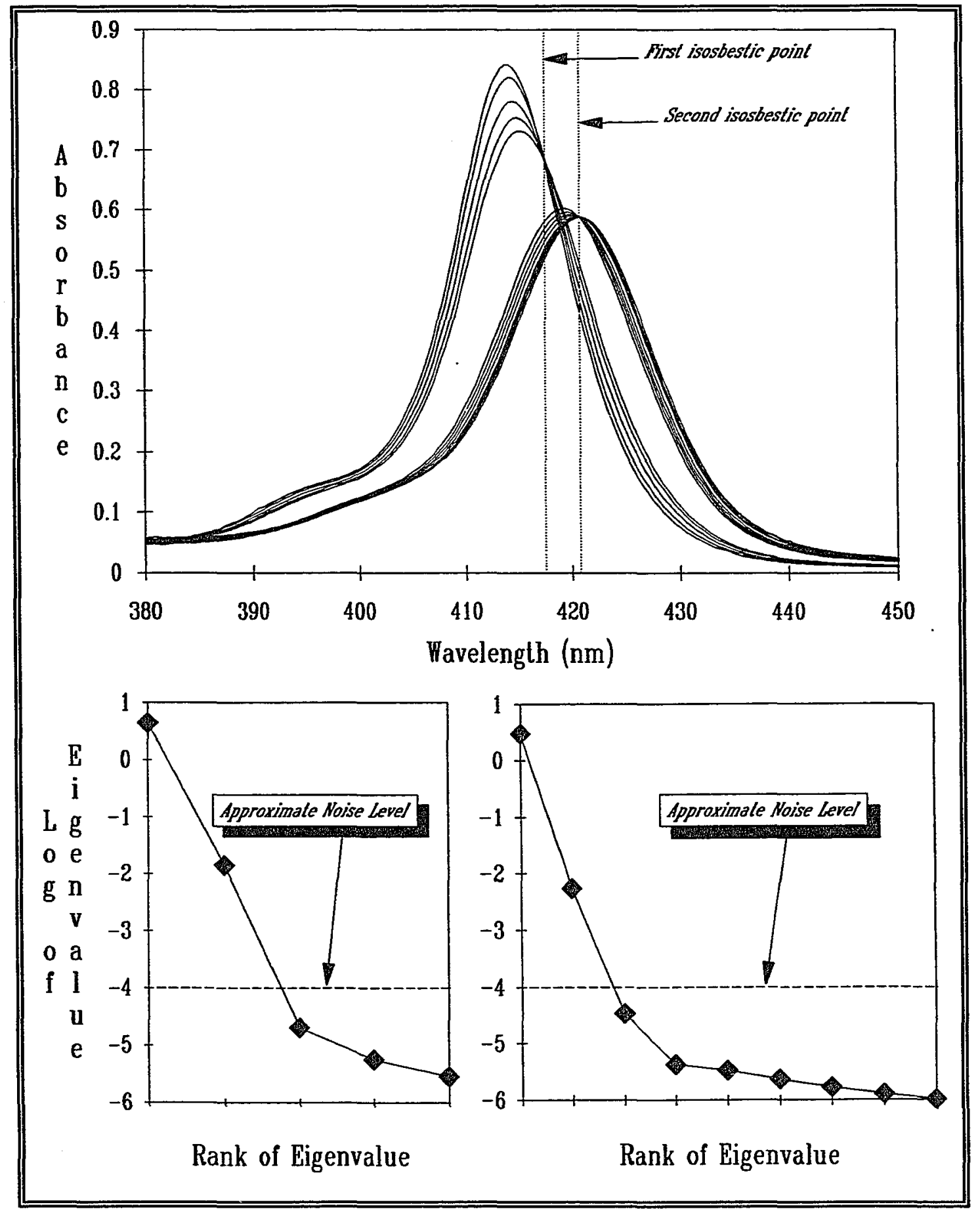

Figure 48. Spectra which pass through the first and second isosbestic points with their corresponding eigenvalue plots. top, selected spectral data, bottom left and right, eigenvalue plot from the first and second isosbestic regions, respectively. Data are selected from experiment 2 . 
regions are projections of the data onto this plane with coordinates $\left(v_{i} \cdot e_{1}, v_{i} \cdot e_{2}\right)$, where $v_{i}$ is the ith absorbance spectrum, $e_{1}$ is the eigenvector associated with the largest eigenvalue, and $e_{2}$ is the eigenvector associated with the second largest eigenvalue. Also indicated is the "most likely" locus for the two components. The first component $\left(\mathrm{H}_{2} \mathrm{TCPP}^{4-}\right)$ must be very near the most dilute spectrum hence near the inner edge of the wedge, i.e. the spectrum and absorptivity of monomer $\mathrm{H}_{2} \mathrm{TCPP}^{4-}$ is nearly resolved; however, remember that the initial spectrum (no viologen) does not pass through the isosbestic thus cannot be used in this analysis. The second component should have a spectral line shape which most closely matches the corresponding component from the second isosbestic point (since they are one and the same). Thus, the pair of data sets with isosbestic points offers a binocular view on the second component spectral line shape. Indeed, we found that a pair of rays could be located for the second component so as to achieve a remarkable congruence of the monocomplex spectral line shapes as shown in Figure 50 (top).

Figure 49 (bottom) shows the corresponding plot for the second isosbestic point indicating the locus of the upper "most likely" component whose spectral shape most closely matches the second component of the first isosbestic mentioned above. The lower component is thought to be near the spectrum of largest concentration (near the inner edge of the wedge).

Figure 50 (top) shows the resulting spectral line shapes of the three components normalized to unit area. The wavelength scale was selected to illustrate the match of the middle component which corresponds to the spectroscopically unresolved $\mathrm{H}_{2} \mathrm{TCPP}^{4-}: \mathrm{MV}^{2+} 1: 1$ association complex.

Absorptivities can be derived from the spectral line shapes of the components if the spectrum of monomeric $\mathrm{H}_{2} \mathrm{TCPP}^{4-}$ (the spectrum containing no $\mathrm{MV}^{2+}$, and its concentration are known. The other two 


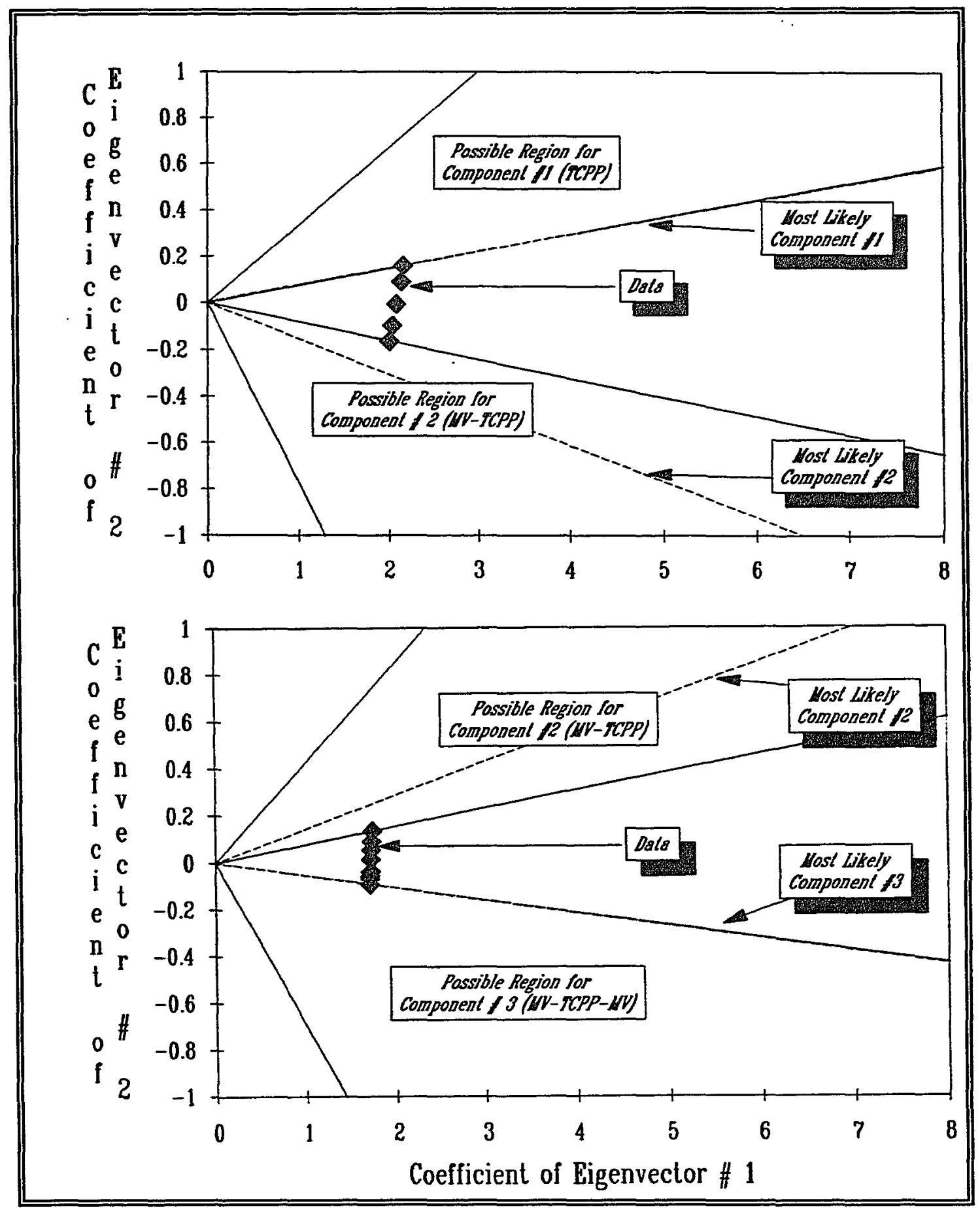

Fiqure 49 Eigenvector coefficient plot used to derive the line shapes and absorptivities for $\mathrm{H}_{2} \mathrm{TCPP}^{4-}$ and the $\mathrm{B}_{2} \mathrm{TCPP}^{4-}$ $: \mathrm{MV}^{2+}$ complexes. The data are from experiment 2 , analysis of spectra passing through the first isosbestic point (top) and the second isosbestic point (bottom) are shown. 


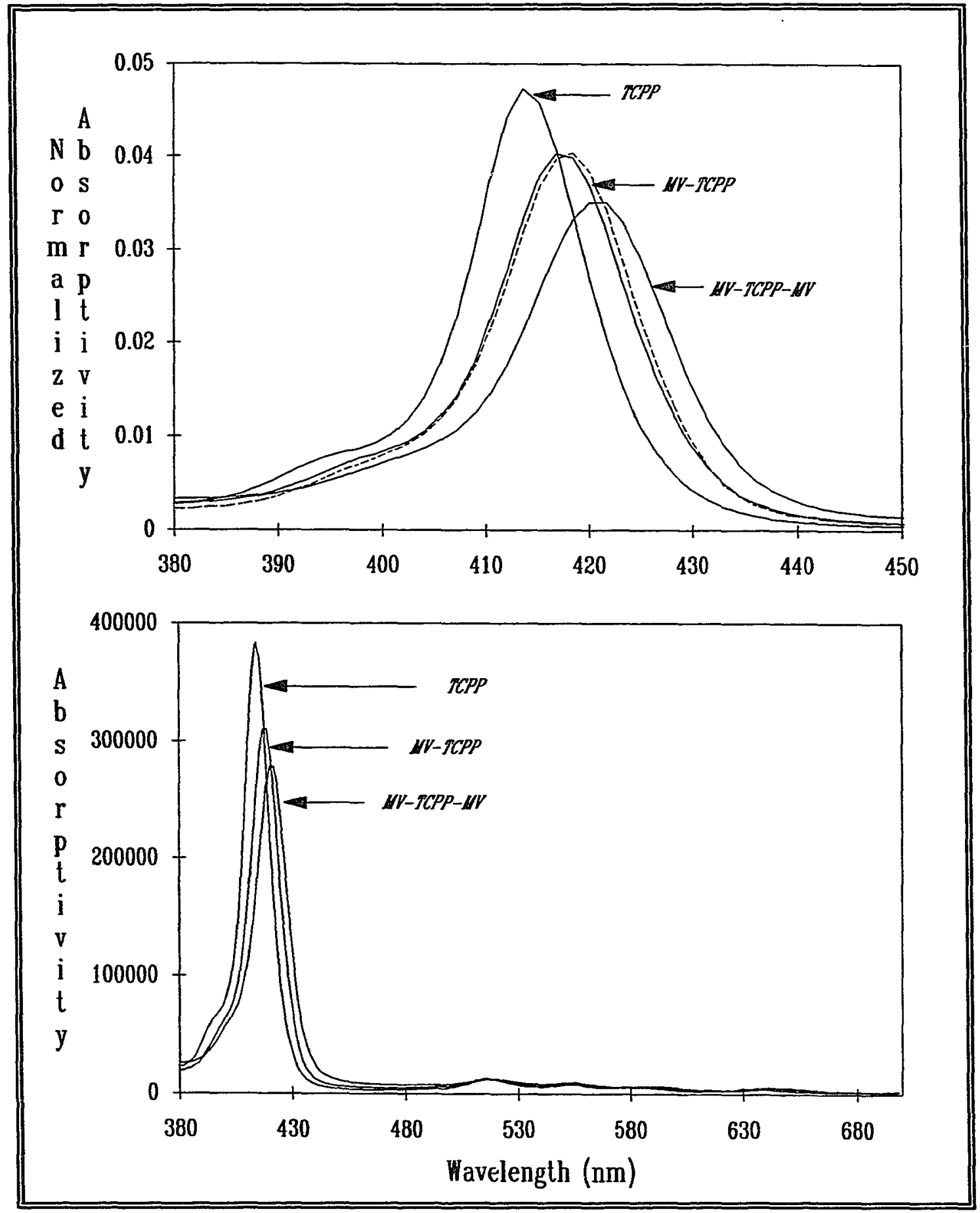

Figure 50. Spectral line shapes of the three absorbing components in experiment 2 from PCA analysis. $\mathrm{MV}^{2+}$ and H $_{2}$ CPP $^{4-}$ are abbreviated as MV and TCPP, respectivelY . Top, Iine shapes are arbitrarily normalized to unit area. Bottom, absorptivities shown on the full wavelength range. 
components are then scaled such that the curves cross at the observed isosbestic points. Figure 50 (bottom) shows the resulting absorptivities after scaling the curves by this method.

Once the absorptivities of the three absorbing species have been derived, the solution concentrations can be found directly by straight multi-variable linear regression. Explicitly, each spectrum from the titration is a linear combination of the spectra of the three components; therefore, at each $\mathrm{MV}^{2+}$ concentration studied, the observed spectrum was decomposed into its component spectra. Since the absorptivities of each of the components have been determined from the principal component analysis, the solution concentration of each can be determined directly. The results in the form of a speciation plot are compared in Figure 51, from both PCA and NLIS analysis (which was shown in Figure 42). The markers show the results of PCA and the curves show the results of nonlinear least-squares analysis. Note the very good agreement between the two methods which lends support to the model of equilibrium equations ( 2 and 3 ) defined in Table XI.

\section{Analysis by PCA, Constant Ionic Strength}

Experiment 3. The example presented above was a "test case" for the data acquired at constant ionic strength shown in Figure 42 . Unlike the data in experiments 1 and 2, which were readily fit by NLLS analysis yielding plausible values for all parameters and acceptable agreement between individual experiments, the data acquired at constant ionic strength were extremely poorly behaved for reasons outlined previously.

Figure 52 shows the eigenvalue plot of the 48 spectra which comprise this data set. There are clearly three components standing out above the noise level, indicating three principal components in solution over the course of the titration analogous to that observed for experiment 2 .

The spectra passing through the first and second isosbestic points with their corresponding eigenvalue plots are shown in Figure 53; notice 


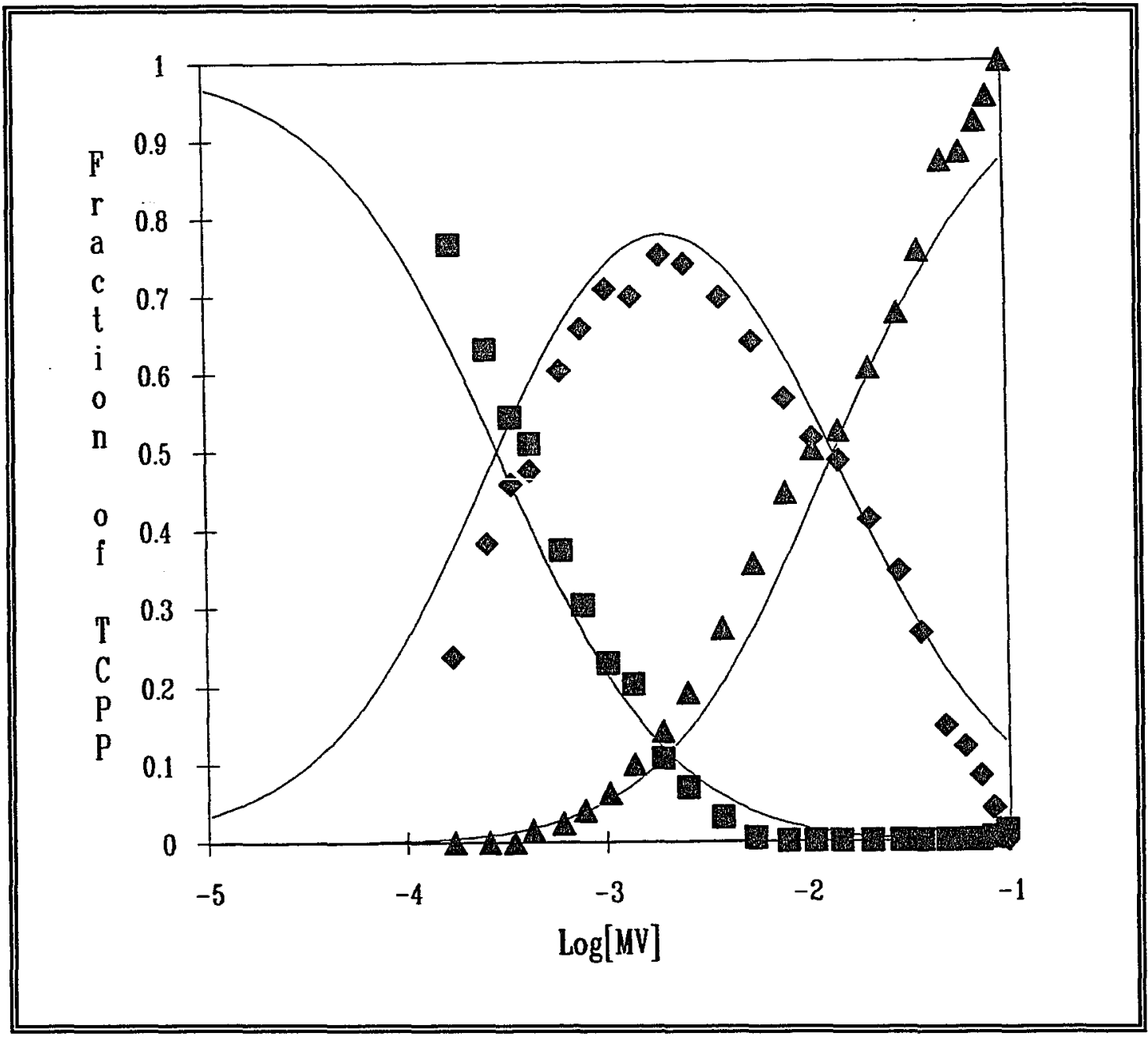

Figure 51. Speciation plot comparing the PCA with the NLIS analysis for experiment 2 . Iines represent the fraction of each component in solution calculated from the association constants determined bY NTLS analysis; markers represent values calculated directly from PCA where the fraction of each component in solution is represented by: $=H_{2}$ TCPP $^{4-}$, $-=\mathrm{H}_{2} \mathrm{TCPP}^{4-}-\mathrm{MV}^{2+}$, and $\Delta=\mathrm{MV}^{2+}-\mathrm{H}_{2} \mathrm{TCPP}^{4-}-\mathrm{MV}^{2+}$.

that in each case only two species are in solution within these regions. Figure 54 shows the eigenvector coefficient plots with the indicated "most probable" loci for each component. Since a greater number of concentration points were included in the analysis, and since the monochrometer error was less, the noise level is lower than that obtained for experiment 2 . 


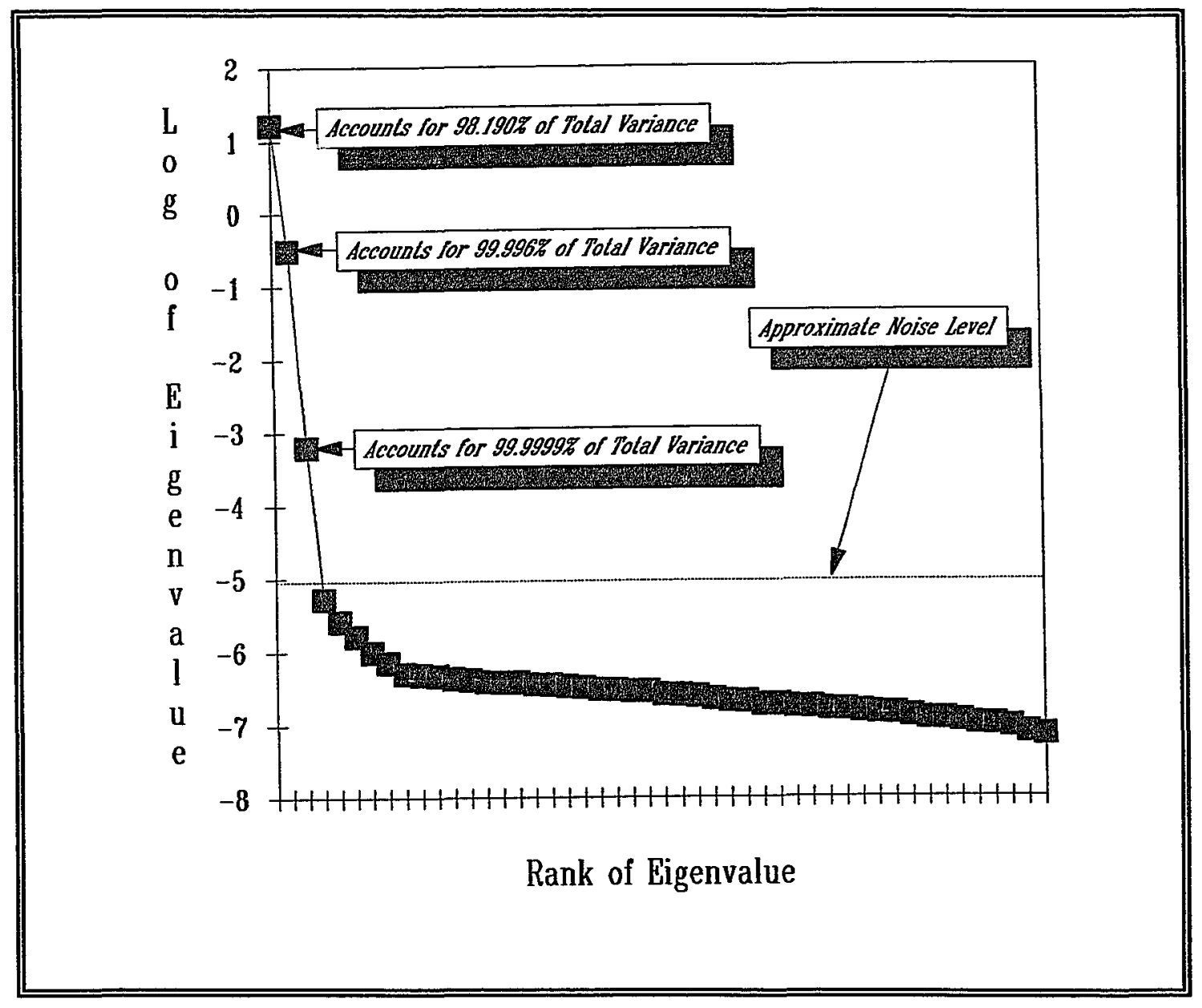

Fiqure 52. Three principal components in the spectral data from experiment 3. Results from the PCA analysis of 48 spectra, 200 absorbance data points/spectra. Absorbance data points at equally spaced wavelengths were selected in the wavelength region from 380 to $450 \mathrm{~nm}$.

The PCA analysis yields additional and independent confirmation that the increased buffer concentration induces some phenomenon other than porphyrin dimerization, such as a spectral line shape change. If the porphyrin dimer equilibrium existed in experiments 2 and/or 3 , it is highly unlikely that this species would have gone undetected in the principal component analysis. The result would have been four principal components, rather than the three which were observed.

It is of interest that not only the absorptivity of the porphyrin 


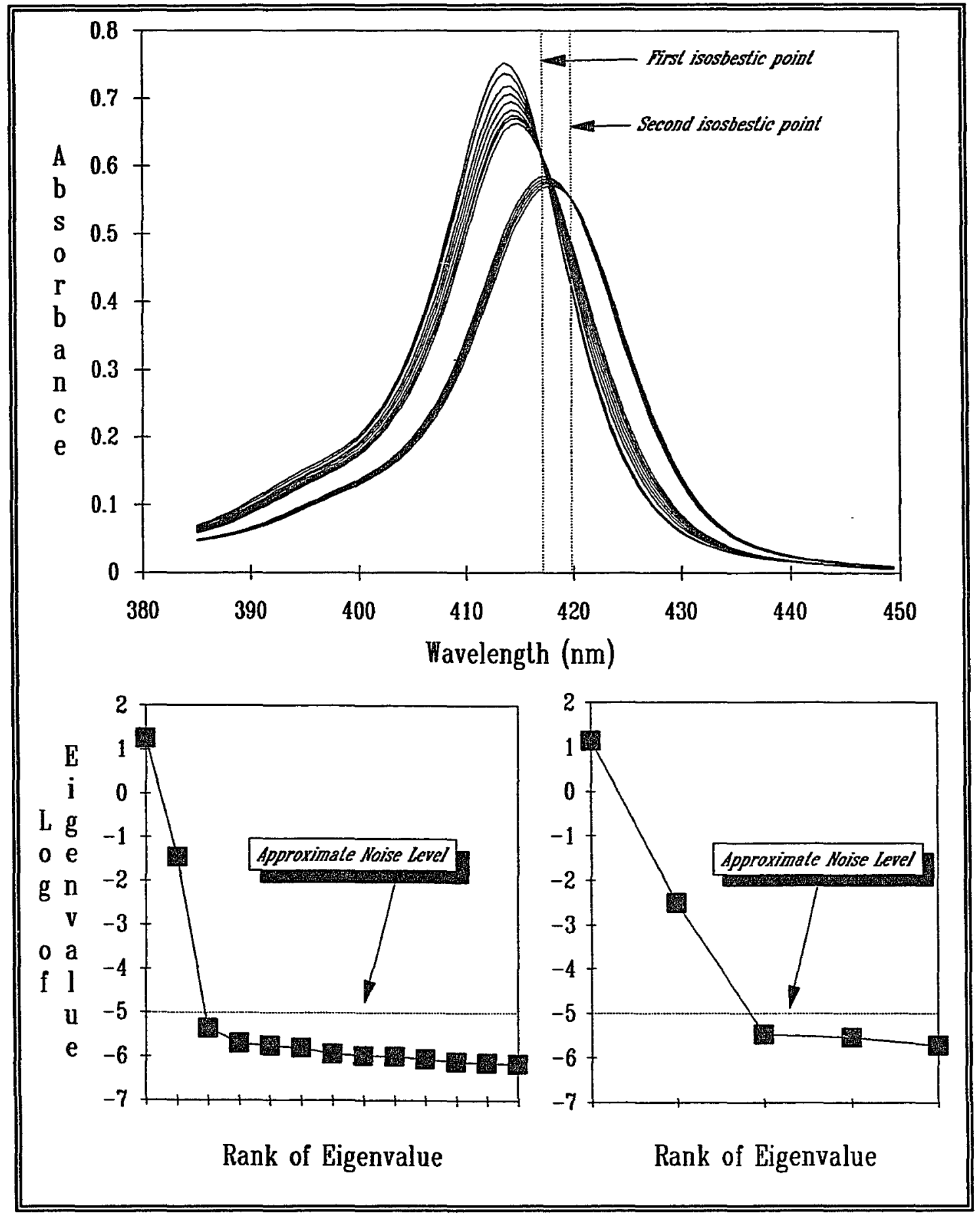

Figure 53. Spectra which pass through the first and second isosbestic points with their corresponding eigenvalue plots. top, selected spectral data, bottom left and right, eigenvalue plot from the first and second isosbestic regions, respectively. Data are selected from experiment 3 . 


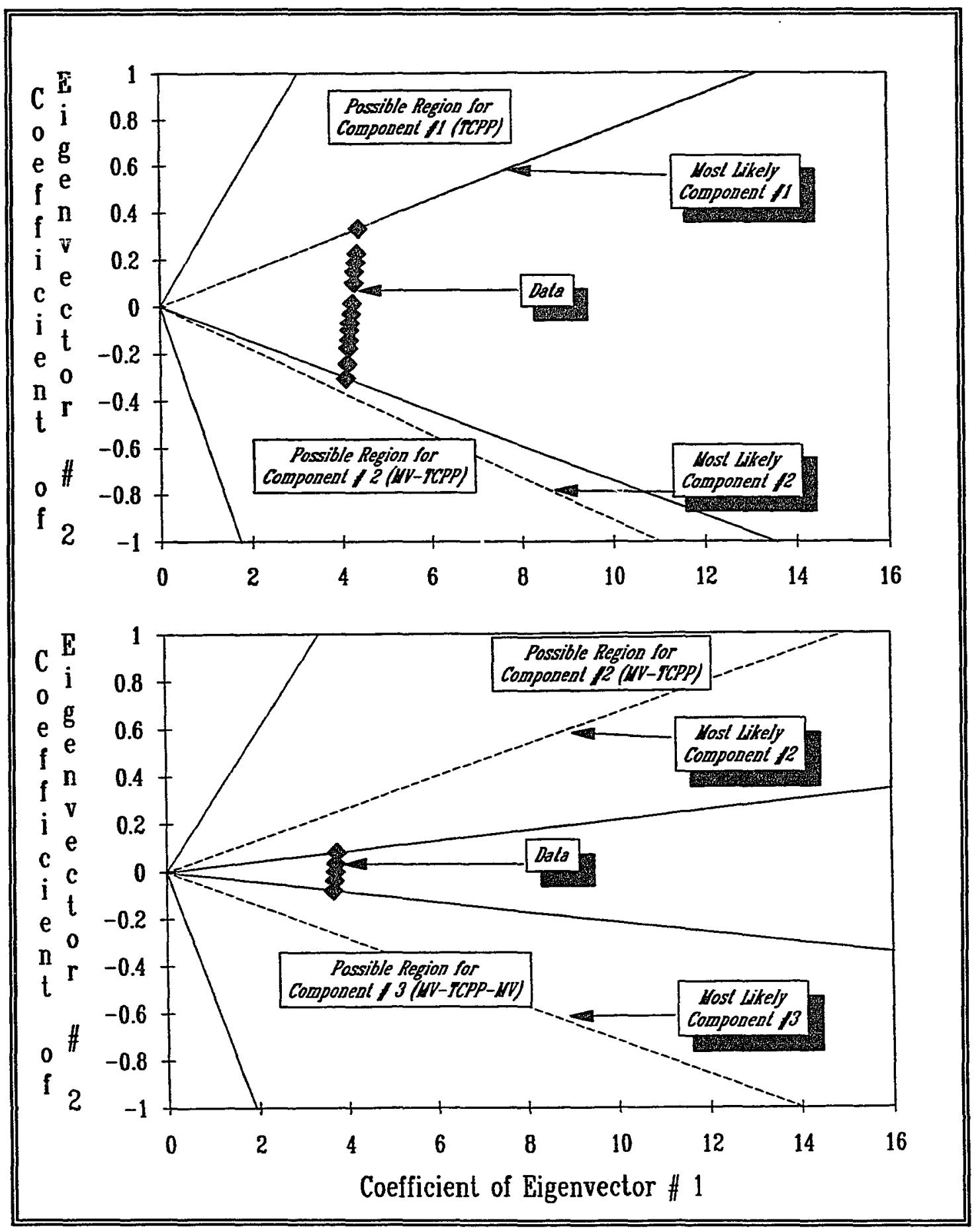

Figure 54. Eigenvector coefficient plot used to derive the line shapes and absorptivities for $\mathrm{B}_{2} \mathrm{TPP}^{4^{-}}$and the , $_{\text {TCPP }}$ 4- $^{-}$ $\mathrm{MV}^{2+}$ complexes. The data are from experiment 3 , analysis of spectra passing through the first isosbestic point (top) and the second isosbestic point (bottom) are shown. 
monomer, but also the absorptivity of the porphyrin/viologen association complexes are strongly dependent upon the buffer concentration. The upper graph in Figure 55 shows the normalized spectral line shapes derived from PCA; in the lower part of this figure we compare the absorptivities of the three components obtained for experiment 3 with those obtained for experiment 2. The spectrum of the $1: 1$ and $1: 2$ association complexes observed at constant ionic strength are both blueshifted, broadened and weakened relative to the analogous complexes observed at constant (low) buffer concentration. The broadening and weakening is consistent with our observations in Cuapter IV, that the absorptivity of $\mathrm{H}_{2} \mathrm{TCPP}^{4-}$ is strongly dependent upon the alkali metal cation concentration due to a buffer-induced spectral line shape change. It is interesting that the spectra of the porphyrin/viologen complexes are more blue-shifted than the porphyrin monomer (relative to the spectra of the complexes and monomer at $5 \mathrm{mM}$ phosphate).

In Figure 56 the speciation plot comparing the results from the PCA and NLLS analysis of experiment 3 is shown. It is immediately apparent that the results from the two analyses do not agree. In the following discussion I will rationalize the source for the discrepancy between values calculated by the two methods and the reason why we trust the values calculated via PCA over those calculated via NLLS.

Table XIII presents a summary of the association constants as determined by each of the two methods, PCA and NLLS, for experiments 1, 2, and 3. Equilibrium constants calculated from PCA yield an individual value for each viologen concentration. When the calculated value is plotted versus the viologen concentration the expected constant function with value equal to the equilibrium constant is obtained for experiment 2. For experiment 3 an unexpected result is obtained, the equilibrium "constant" is again linear when plotted versus the viologen concentration; however, the slope for $\mathrm{K}_{2}$ is positive indicating that as the buffer is diluted, the equilibrium is shifted towards higher 


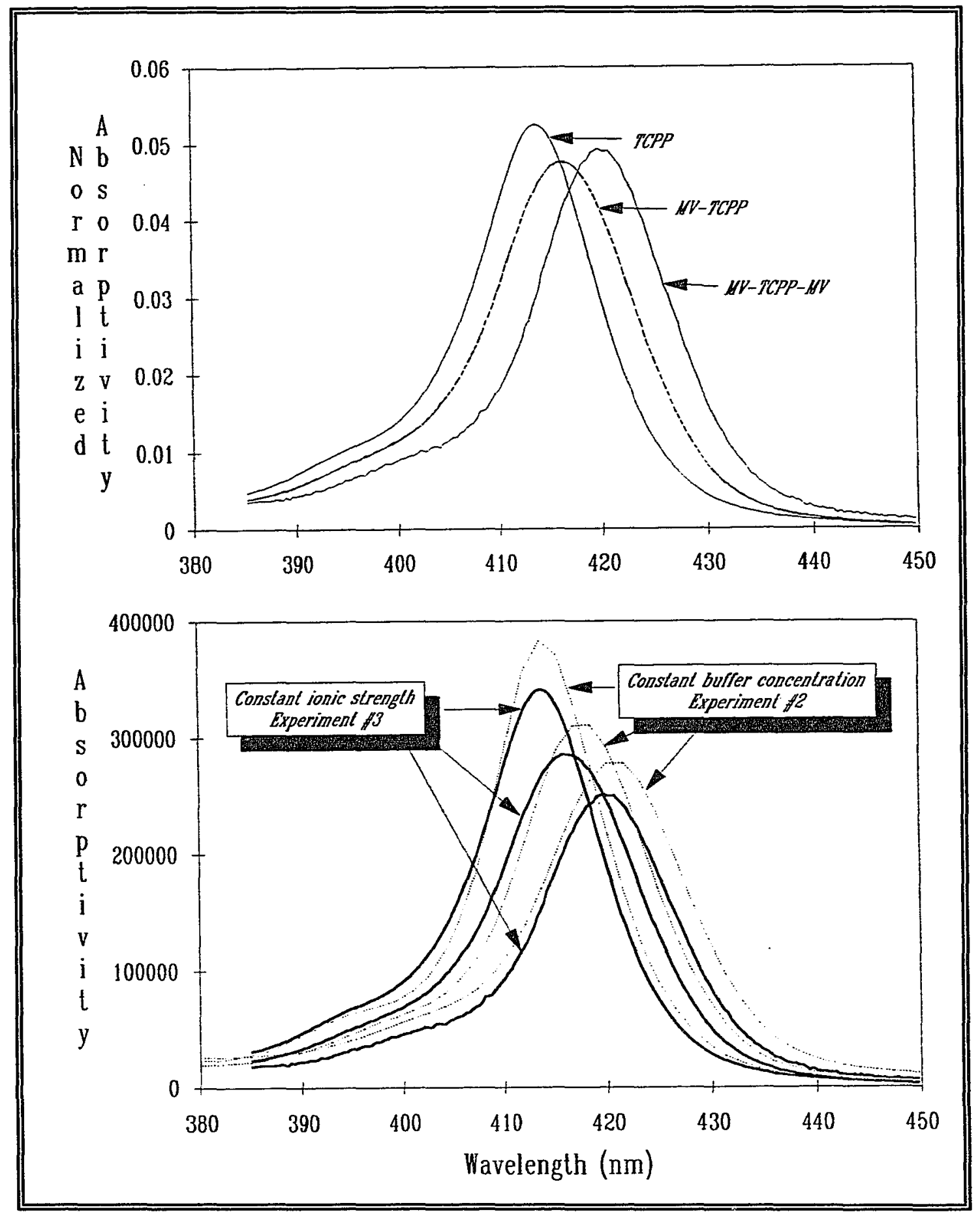

Figure 55. Spectral line shapes of the three absorbing components in experiment 3 from PCA analysis. MV $^{2+}$ and H $_{2}$ TCPP $^{4-}$ are abbreviated as MV and TCPP, respectivelY. Top, Iine shapes are arbitrarily normalized to unit area. Bottom, absorptivities are compared with experiment 2 . 


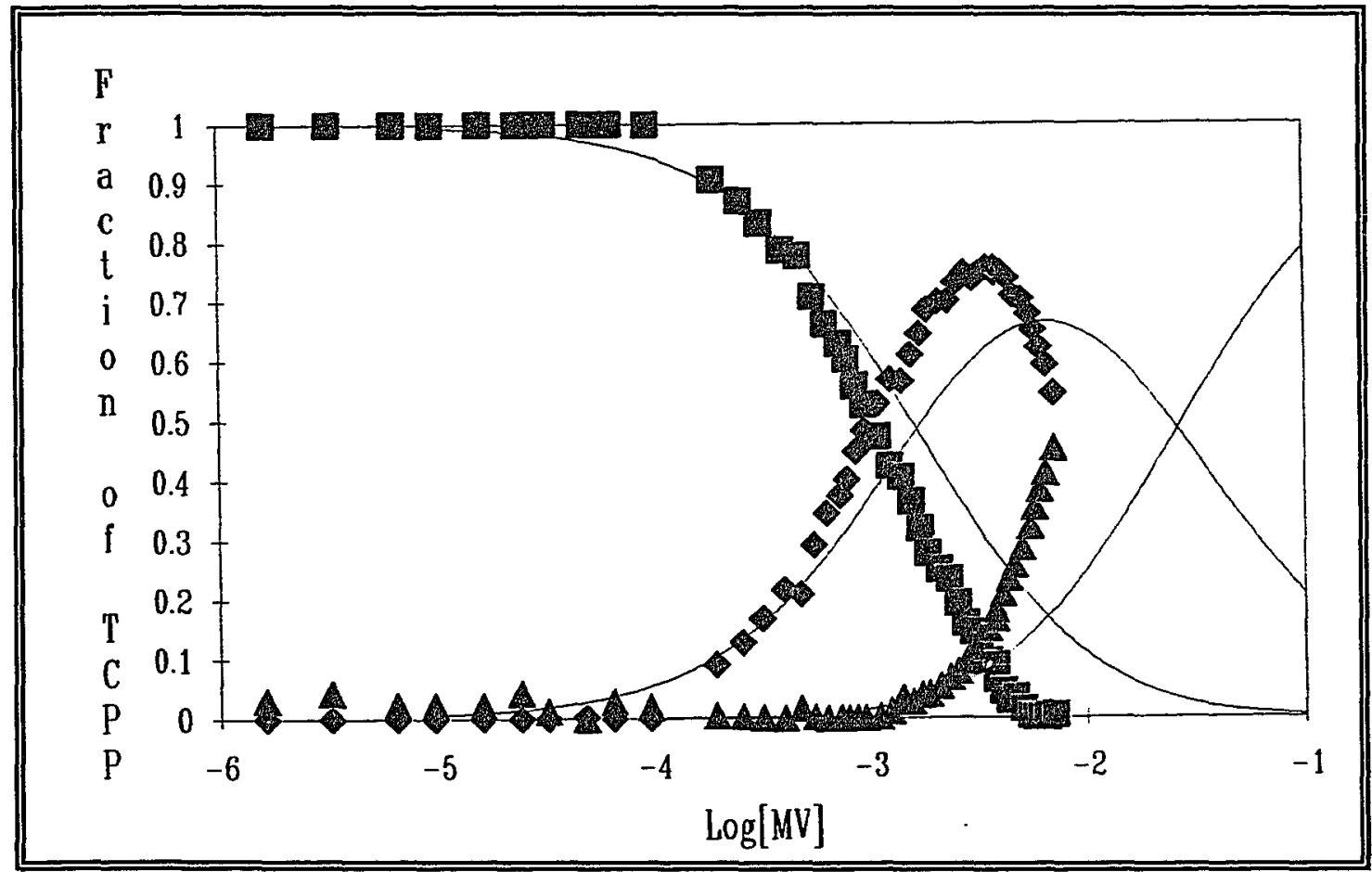

Figure 56. Speciation plot comparing the PCA with the NIIS analysis for experiment 3 . Lines represent the fraction of each component in solution calculated from the association constants determined by MLS analysis; markers represent values calculated directly from PCA where the fraction of each compopent in solution is represented by: $1=\mathrm{E}_{2} \mathrm{TCPP}^{4-}$, $\checkmark=\mathrm{H}_{2} \mathrm{TCPP}^{4-}-\mathrm{MV}^{2+}$, and $\Delta=\mathrm{MV}^{2+}-\mathrm{H}_{2} \mathrm{TCPP}^{4-}-\mathrm{MV}^{2+}$.

TABLE XIII

EQUILIBRIUM CONSTANTS CALCULATED BY NLIS ARD PCA

\begin{tabular}{|c|c|c|c|c|}
\hline \multirow{2}{*}{$\begin{array}{l}\text { Experiment } \\
\text { Description }\end{array}$} & \multicolumn{2}{|c|}{$\begin{array}{l}\text { Equilibrium } \\
\text { Constant } \mathrm{K}_{1}\end{array}$} & \multicolumn{2}{|c|}{$\begin{array}{l}\text { Equilibrium } \\
\text { Constant } \mathrm{K}_{2}\end{array}$} \\
\hline & NLIS & $\mathrm{PCA}$ & NLLS & $\mathrm{PCA}$ \\
\hline $\begin{array}{c}\text { Constant Buffer } \\
\text { Experiment } 1\end{array}$ & $3171 \pm 47$ & N.A. & $108 \pm 5$ & N.A. \\
\hline $\begin{array}{c}\text { Constant Buffer } \\
\text { Experiment } 2\end{array}$ & $3350 \pm 260$ & $2200 \pm 410$ & $68 \pm 7$ & $100 \pm 2$ \\
\hline $\begin{array}{c}\text { Constant Ionic } \\
\text { Strength }\end{array}$ & $594 \pm 150$ & $1217 \pm 350$ & $38 \pm 11$ & $140 \pm 21$ \\
\hline
\end{tabular}


values (shifted towards lower viologen concentrations). The slope obtained for $\mathrm{K}_{1}$ was nearly zero, thus the reported value is a constant; in order to report one number for $k_{2}$ we averaged the values determined within the viologen concentration region containing the second isosbestic point. The variable equilibrium "constant" observed for $\mathrm{K}_{2}$ in experiment 3 can be explained by the greater incremental dilution of the buffer within this viologen concentration range. The observation that $k_{2}$ is not, in fact, constant at constant ionic strength implies that the overall ionic strength is less important than the absolute buffer concentration in determining the equilibrium constant.

In the NLLS analysis the model assumes that each equilibrium constant can be described as a single value which explains the failure of this method at constant ionic strength. In contrast, the spectral line shapes and absorptivities derived from principal component analysis appear to be reasonable and consistent with our model concerning the effect of two experimental variables, buffer and viologen, on both the spectra and equilibrium constants. In addition, the values are seen to be consistent in relative magnitude with other porphyrin studies summarized in Table $x_{i}$ although the presence of a complex with stoichiometry higher than 1:1 has not been previously recognized. At higher salt concentrations the equilibrium is shifted towards complex dissociation which is also in agreement with the literature.

It is apparent from this work that the effect of the buffer on the spectra and speciation of anionic tetraphenylporphyrins is a subject which is little understood, and deserves more attention. It appears that the equilibrium constants are dependent upon the cation concentration in ways more complex than can be corrected by the DebyeHückel equation. This means that the constants are only valid under the same conditions which they were measured. This principle also applies to porphyrin absorptivities and may indicate a reevaluation of some of the reported data in the literature. 


\section{SELECTED REFERENCES}

1. Nielands, J.B. In Metal Ions in Biological Systems, "Chemistry of Iron in Biological Systems"; Dhar, Ed.; Academic Press: New York, 1974, and references therein.

2. Williams, R. J. P. In Iron in Biochemistry and Medicine, "Haemproteins and Oxygen"; A. Jacobs and M. Wormwood eds.; Academic Press: New York, 1974, and references therein.

3. Taylor, G. A.; Tsutsui, M. J. Chem. Ed. 1975, 52, 715, and references therein.

4. Gray, H. B. Presented at Symposium on Biological Aspects of Inorganic Chemistry, University of British Columbia, Vancouver, $B$. C., June 20-25, 1976.

5. Williams, R. J. P. Presented at Symposium on Biological Aspects of Inorganic Chemistry, University of British Columbia, Vancouver, B. C., June 20-25, 1976.

6. Fenna, R. E.; Matthews, B. W. In The Porphyrins; Dolphin, D., Ed.; Academic: New York, 1978; Vol. VII, Part $B$, and references therein.

7. Griffin, B. W.; Peterson, J. A.; Estabrook, R. W. In The Porphyrins; Dolphin, D., Ed.; Academic: New York, 1978; Vol. VII, Part $B$, and references therein.

8. Clayton, R. K. In Tunneling in Biological Systems, 1979, Chance, B. ed. Academic Press, New York, 377.

9. Winkler, J. R.; Nocera, D., G.; Yocom, E. B.; Gray, H. B. J. Am. Chem. Soc. 1982, 104, 5798.

10. Iikhtenstein, G. I.; Kulikow, A. V.; Kotelnikov, A. I. Photobiochem. Photobiophys. 1982, 3, 337.

11. Siders, P.; Cave, R. J.; Marcus, R. A. J. Chem. Phys. 1984, 81, 5613.

12. Govindjee; Kambara, T.; Coleman, พ. Photochem. Photobiol. 1985, 42, 187. And references therein.

13. Lavalee, D. K. Coord. Chem. Rev. 1985, 61, 55.

14. Cave, R. J.; Siders,P.; Marcus, R. A. J. Phys. Chem. 1986, 90, 1436.

15. Khairutdinov, R. F.; Brickenstein, E. K. Photochem. Photobiol. 1986, 43, 339. And references therein.

16. Karas, J. K.; Lieber, C. M.; Gray H. B. J. Am. Chem. Soc. 1988, $110,599$.

17. Thomas, D. W.; Martel, A. E. J. Am. Chem. Soc. 1956, 78, 1335. 
18. Thomas, D. W.; Martel, A. E. J. Am. Chem. Soc. 1956, 78, 1338.

19. Adler, A. D.; Longo, F. R.; Finarelli, J. D.; Goldmacher, J.; Assour, J.; Korsakoff, I. J. Org. Chem. 1966, 1967.

20. Treibs, A.; Haberle, N. Liebigs Ann. Chem. 1968, 718, 183-207.

21. Collman, J. R.; Gagne, R. R.; Halbert, T. R.; Marchon, J.; Reed C. A. J. Am. Chem. Soc. 1973, 95, 7868 .

22. Collman, J. P.; Gagne, R. R.; Reed C. A.; Halbert, T. R.; Lang, G.; Robinson, W. T.; ; J. Am. Chem. Soc., 1975, 97, $142 \%$.

23. Kim, J. B.; Adler, A. D.; Longo, F. R. In The Porphyrins; Dolphin, D., Ed.; Academic: New York, 1978; Vol.1, and references therein.

24. White, W. I.; Bachmann, R. C.; Burnham, B. F. In The Porphyrins; Dolphin, D., Ed.; Academic: New York, 1978; Vol.1, and references therein.

25. Bogat-skii, A. V.; Zhilina, Z. I.; Krasnoshchekaya, S. P.; Zakharova, R. M. Zhurnal Organicheskoi Kimii 1982, 18, 2304.

26. Collman, J. P.; Anson, F. C.; Barnes, C. E.; Bencosme, C. S.; Geiger, T.; Evitt, E. R.; Kreh, R. P.; Meier, K.; Pettman, R. B. J. Am. Chem. Soc. 1983, 105, 2694.

27. Dorough, G. D.; Miller, J. R.; Huennekens, F. M. J. Am. Chem. Soc. $1951,73,4315$.

28. Gouterman, M. In The Porphyrins; Dolphin, D., Ed.; Academic: New York, 1978; Vol. III, Part $A$, and references thereir.

29. Hopf, F. R.; Whitten, D. G. In The Porphyrins; Dolphin, D., Ed.; Academic: New York, 1978; Vol.II, Part B, and references therein.

30. Adar, F. In The Porphyrins; Dolphin, D., Ed.; Academic: New York, 1978; Vol.III, Part $A$, and references therein.

31. Southerland, J. C. In The Porphyrins; Dolphin, D., Ed.; Academic: New York, 1978; Vol.III, Part $A$, and references therein.

32. Austin, E.; Gouterman, M. Bioinorg. Chem., 1978, 9, 281.

33. San Pietro, A. In Microbial Energy Conversion, 1977, Schlegel, H. G. and Barnea J., eds. Pergamon Press, 235-243.

34. Henglein, A. In Photochemical Conversion and storage of solar Energy; Rabani, J. Ed.; Weizmann Science Press of Isreal, 1982; Part $A$, and references therein.

35. Kalyanasundaram, K.; Neumann-Spallart, M. J. Phys. Chem. 1982, $86,5163$.

36. Harriman, A.; Richoux, M.C.; Neta, P. J. Phys. Chem. 1983, 87, 4957.

37. Bardwell, J. Bolton, J. R. Photochem. Photobiol. 1984, 39, 735.

38. Kadish, K. M.; Sazou, D.; Iiu, Y. M.; Saoiabi, A.; Ferhat, M.; Guilarả, R. Inorg. Chem. 1988, 27, 686 .

39. Neta, P. J. Phys. Chem., 1981, 85, 3678.

40. Shelnutt, J. A. J. Am. Chem. Soc., 1983, 105, 7179. 
41. Harriman, A.; Porter, G.; Wilowska, A. J. Chem. Soc., Faraday Trans. 2 1983, 79, 80́7.

42. Smalley, J. F.; Feldberg, S. W.; Brunschwig, B. S.; J. Phys. Chem, 1983, 87, 1757.

43. McLendon, G.; Miller, D. S. J. Chem. Soc., Chem. Commun. 1980, 533.

44. Schmehl, R. H.; Whitten, D. G. J. Phys. Chem. 1981, 85, 3473.

45. Brugger, P.-A. Grätzel, M; Guarr, T.; McLendon, G. J. Phys. Chem. $1982,86,944$.

46. Rougee, M.; Ebbesen, T.; Ghetti, F.; Bensasson, R. V. J. Phys. Chem. 1982, 86, 4404 .

47. Okura, I.; Aono, S.; Takeuchi, M.; Kusunoke, S.; Bull. Chem. Soc. Jpn. $1982,55,3637$.

48. Richoux, M.; Harriman, A. J. Chem. Soc., Faraday Trans. I 1982, $78,1873$.

49. Kalyanasundaram, K. J. Chem. SoC. Faraday II, 1983, 79, 1365.

50. Okura, I.; Kusunoke, S.; Aono, S. Inorg. Chim. Acta 1983, 77, I99.

51. Okura, I.; Kusunoke, S.; Aono, S. Inorg. Chem. 1983, 22, 3828.

52. Kano, K.; Sato, T.; Yamada, S,; Ogawa, T. J. Phys. Chem. 1983, $87,566$.

53. Sato, T.; Ogawa, T.; Kano, K. J. Phys. Chem. 1984, 88, 3678.

54. Harriman, A.; Porter, G.; Wilowska, A. J. Chem. Soc., Faraday Trans. 2 1984, 80, 191.

55. Harriman, A. Inorg. Chim. Acta 1984, 88, 213.

56. Nahor, S.G.; Rabani, J. J. Phys. Chem. 1985, 89, 2468.

57. Aono, S.; Okura, I.; Yamada, A. J. Phys. Chem. 1985, 89, 1593.

58. Kano, K.; Nakajima, T.; Hashimoto, S. J. Phys. Chem. 1987, 91, 6614 .

59. Zachariasse, K. A.; Whitten, D. G. Chem. Phys. Lett. 1973, 22, 527.

60. Pasternack, R. F.; Spiro, E. G.; Teach, M. J. Inorg. Nucl. Chem. $1974,36,599$.

61. Chang, C. K. J. Heterocycíic Chem. 1977, 14, 1285.

62. White, W. I.; Bachmann, R. C.; Burnham, B. F. In The Porphyrins; Dolphin, D., Ed.; Academic: New York, 1978; Vol. V, Part $C$, and references therein.

63. Kano, K.; Miyake, T.; Uomoto, T.; Sato, T.; Ogawa, T.; Hashimoto, S. J. Chem. Lett. 1983, 1867. 64. Ojadi, E.; Selzer, R.; Linschitz, H. J. Am. Chem. Soc. 1985, 107,
7783.

65. Miller, A.; Knoll, W.; Mohwald, H.; Ruaudel-Teixier, A.; Thin Solid Films, $1985,133,83$.

66. Brookfield, R. L.; Ellul, H.; Harriman, A. J. Photochem. 1985, 31, 97. 
67. Hofstra, U.; Koehorst, R. B. M.; Schaafsma, T. J. Chem. Phys. Lett. $1986,130,555$.

68. Leighton, P.; Cowan, J. A.; Abraham, R. J.; Sanders, R. M. J. Org. Chem. 1988, 53, 733.

69. Firman, P.; Wilkins, R. G.; Kasprzyk, S. P. J. Am. Chem. Soc. 1989, 111, 4990 .

70. Gregg, B. A.: Fox, M. A.; Bard, A. J. J. Am. Chem. Soc. 1989, 93, 4227, and references therein.

71. C. C. Wamser, R. R. Bard, V. Senthilathipan, V. C. Anderson, J. A. Yates, H. K. Ionsdale, G. W. Rayfield, D. T. Friesen, D. A. Lorenz, G. C. Stangle, P. V. Eikeren, D. R. Baer, R, A. Kansdell, J. H. Golbeck, W. C. Babcock, J. J. Sandberg, and S. E. Clarke, J. Amer. Chem. Soc., 1989, 111, 8485.

72. Ishii, H.; Satoh, K.; Satoh, Y.; Koh:. H. Talanta 1982, $29,545$.

73. Corsini, A.; Difruscia, R.; Herrmann, O. Talanta 1985, 32, 791.

74. Cauzzo, C.; Gennari, G.; Jori, G.; Spikes, J. D. Photochem. Photobiol. 1977, 25, 389.

75. Grossweiner, I. I.; Patel, A.S.; Grossweiner, J. B. Photochem. Photobiol. 1982, 36, 159.

76. Dixit, R.; Mukhtar, H.; Bickers, D. R. Photochem. Photobiol. 1983, $36,173$.

77. Reddi, E.; Rodgers, M. A. J.; Spikes, J. D.; Jori, G. Photochem. Photobiol. 1984, 40,415 .

78. Praseuth, D.; Gaudemer, A.; Verlhac, J. B.; Kraljic, I.; Sissoeff, I.: Guille, E. Photochem. Photobiol. 1986, 44, 717.

79. Seret, A.; Gandin, E.; van de Vorst, A. Photobiochem. Photobiophys. $1986,12,259$.

80. Lambert, C. R.; Reddi, E.; Spikes, J. D.; Rodgers, M. A. J.; Jori, G. Photochem. Photobiol. 1986, 44, 595.

81. Ghiggino, K.P.; Bennett, L. E.; Henderson, R. W. Photochem. Photobiol. 1988, 47, 65.

82. Davila, J; Harriman, A. J. Am. Chem. Soc. 1990, $112,2686$.

83. Das, R. R. i Pasternack, R. F.; Plane, R. A. J. Am. Chem. Soc. 1970, 92,3312 .

84. Pasternak, R. F.; Ann. N. Y. Acad. Sci., 1973, $206,614$.

85. Pasternack, P.; Huber, R.; Boyd, P.; Engasser, G.; Francesconi, L.; Gibbs, E.; Fasella, P.; Venturo, G. C.; Hinds, L. dec. J. Ám. Chem. Soc. 1972, 94, 4511.

86. Pasternack, R. F.; Sutin, N.; Turner, D. H. J. Am. Chem. Soc. $1976,98,1908$.

87. Rau, W. G.; Longo, F. R. Inorg. Chem. 1977, 16, 1372 .

88. Smalley, J. F.; Feldberg, S. W.; Brunschwig, B. S. J. Phys. Chem. $1983,87,1757$.

89. Corsini, A.; Herrmann, O. Talanta 1986, 33, 335.

90. Torki, F. M.; Casano, P. J.; Reed, W. F.; Schmehl, R. H. J. Phys. Chem. 1987, 91, 3686 . 
91. There are a variety of different reports for pKa's of $\mathrm{H}_{2} \mathrm{TCPP}^{4-}$. The biggest disagreement is found in reference 67 , where they report $\mathrm{H}_{2} \mathrm{TCPP}^{4-} \mathrm{a}$ (singular) pKa for $\mathrm{H}_{2} \mathrm{TCPP}^{4-}$ that is identical to the published value of benzoic acid.

92. Lambert et. al. (80) report $\mathrm{H}_{2} \mathrm{TCPP}^{4-}$ to be in monomer form at $8.4 \times 10^{-5} \mathrm{M}$ at $\mathrm{pH}=7.4$ in ca. $25 \mathrm{mM}$ phosphate, although they do not support this with any experimental evidence. Pasternack and coworkers' observations are similar to ours, i.e. The Beer's law plots for $\mathrm{H}_{2}$ TCPP $^{4-}$ deviate from lineraity above ca. $2 \times 10^{-6} \mathrm{M}$ (our conditions, $5 \mathrm{mM}$ phosphate) at neutrality.

93. Pileni, M.-P.; Braun, A. M.; Grätzel, M. Photochem. Photobiol. $1980,31,423$.

94. Willner, I.; Degani, Y. Israel J. Chem. 1982, 22, 163.

95. Willner, I.; Degani, Y. J. Am. Chem. Soc. 1983, 105, 6228.

96. Watanabe, T.; Honda, K. J. Phys. Chem. 1982, 86, 2617.

97. Nagamura, T.; Kurihara, T.; Matsuo, T.; Sumitani, M.; Yoshihara, K. J. Phys. Chem. 1982, 86, 4368.

98. Venturi, M.; Mulazzani, Q. G.; Hoffman M. 2. Radiat. Phys. Chem. 1984, 23, 229.

99. Ebbesen, T. W.; Manring, L. E.; Peters, K. S. J. Am. Chem. Soc. $1984,106,7400$.

100. Ford, W. E.; Tollin, G. Photochem. Photobiol. 1986, 43, 467.

101 Baumgartner, E.; Bertolotti, S.G.; Cosa, J.J.; Gsponer, H.E.; Hamity, M.; and Prevital, C. M. Journal of Colloid and Interface Science 1986, 15, 417.

102. Kawanishi, Y.; Kitamura, N.; Tazuke, S.; J. Phys. Chem. 1986, 90, 2469 .

103. At $\mu \mathrm{M}$ concentrations of $\mathrm{H}_{2} \mathrm{TCPP}^{4-}$, the cation exchange properties of the glass can cause significant experimental error. We observe this primarily with alkali metal ions, although we have also observed that cationic porphyrins can bind to glassware and are released in the presence of organic solvents or anionic porphyrins (discussed further in later chapters). Other authors (81) report the time-dependent formation of metallo-porphyrins (due to the chelation of metals from the surface of contaminated cuvettes).

104. Harvey, C. E. In Semiquantitative Spectrochemistry 1964.

105. Lawton, W. H.; Sylvestre, E. A. Technometrics 1971, 13, 617.

106. Ohta, N. Anal. Chem. 1973, 45, 553.

107. Malinowski, E. R.; Howery, D. G. Factor Analysis in Chemistry; John Wiley \& Sons, Inc.: New York, 1980.

108. Aartsma, T. J.; Gouterman, M.; Jochum, C.; Kwiram, A. L.; Pepich, B. V.; Williams, L. D. J. Am. Chem. Soc. 1982, 104, 6278.

109. Gampp, H.; Maeder, M.; Meyer, C. J.; Zuberbuhler, A. D. Talanta, $1985,32,1133$.

110. Malinowski, E. R. In Computer-Enhanced Analytical Spectroscopy; "Evolutionary Factor Analysis in Analytical Spectroscopy"; Meuzelaar, H. L. C.; Isenhour, T. L., Eds., Plenum Press: New York, 1987. 
111. Russel1,-M. D.; Gouterman, M. Spectrochim. Acta. 1988, 44A, 857.

112. Schostack, K.; Parekh, P.; Patel, S.; Malinowski, E. R. J. Res. Nat. Bur. Standards 1988, 93, 256.

113. Benesi, H. A.; Hildebrand, J. H. J. Am. Chem. Soc. 1949, 71, 2703.

114. Rose, N.; Drago, R. J. Amer. Chem. Soc., 1959, 81, 6138.

115. Conrow, K., Johnson, G. D., Bowen, R. E. J. Am. Chem. Soc. 1964, $86,1025$.

116. Ramette, R. W. J. Chem. Ed. 1965, 44, 647.

117. Deranleau, D. A. J. Am. Chem. Soc. 1969, 91, 4044.

118. Wachter, H. N.; Fried, V. J. Chem. Ed. 1974, 51, 798.

119. Carta, G.; Crisponi, G. J. Chem Soc., Perkin II 1982, 53.

120. Jencks, W. P., Altura, R. A. J. Chem. Ed. 1988, 65, 770.

121. Gouterman, M. Personal communication.

122. Fulton, R. L.; Gouterman M. J. Chem. Phys. 1964, 41, 2280.

123. Yan, X.; Holten, D. J. Phys. Chem. 1988, 92, 409.

124. Hunter, C. A.; Sanders, K. M.; Stone, J. J. Chem. Phys. 1989, 133, 395.

125. Schick, G. A. Schreiman, I. C.; Wagner, R. W.; Iindsey, J. S.: Bocian, D. F. J.Am. Chem. Soc. 1989, 111, 1344.

126. Jaffe, H. H.; Orchin, M. Theory and Applications of Ultraviolet Spectroscopy; John Wiley and Sons, Inc.: New York, 1962.

127. Rocklin, R. D.; Murray, R. W. J. Electroanal. Chem. 1979, 100, 271.

128. Dolphin, D.; Forman, A.; Borg, D. C.; Frajer, J.; Felton, R. H. Proc. Nat. Acad. Sci. 1971, 68, 614.

129. Fajer, J.; Davis, M. S. In The Porphyrins; Dolphin, D., Ed.; Academic: New York, 1978; Vol. IV, Part B, and references therein.

130. Janson, T. R.; Katz, J. R. In The Porphyrins; Dolphin, D., Ed.; Academic: New York, 1978; VoI. IV, Part B, and references therein.

131. La Mar, G. N, ; Walker, F. A. In The Porphyrins; Dolphin, D., Ed.; Academic: New York, 1978; Voyl. IV, part B, and references therein.

132. In The Standard Spectra Collection; Sadtler Research Laboratories, Division of Bio-Rad Laboratories, Inc. most recent update, 1989, For asymmetrically substituted viologens, the NMR spectra numbered: $41395,41396,41399$. For symmetrically substituted viologens: 41397,34325 .

133. Falk, J. E. In Porphyrins and Metalloporphyrins; Elsevier, Amsterdam, 1964.

134. Harvey, C. E. In Semiquantitative Spectrochemistry; Applied research Laboratories, Inc.: Glendale, California, 1964.

135. Hambright, P. Personal communication.

136. Braun, A. M. Personal communication. 
137. Taniguchi, Y.; Makimoto, S.; Suzuki, J. Phys. Chem., 1981, 85, 2218.

138. Press, W. P.; Flannery, B. P.; Teukolsky, S. A.; Vetterling, W. T. Numerical Recipes; Cambridge University Press: New York, 1986.

139. Suppan, P. Photochem. Photobiol., A: Chemistry 1990, 50, 293.

140. Gianneschi, L. P.; Cant, A.; Kurucsev, T. J. Chem. Soc. Faraday II $1977,73,664$.

141. Gal, M.; Kelly, G. R.; Kurucsev, T. J. Chem. Soc. Faraday II 1977, 69,395 .

142. Yamagishi, A.; Watanabe, F.; J. Phys. Chem. 1981, 85, 2129.

143. Thanabal, V.; Krishnan, V. J. Am. Chem. Soc. 1982, 104, 3643.

144. Jones, G. R.; Duddell, D. A.; Murray, D.; Cundall, R. B. J. Chem. Soc., Faraday Trans. II 1984, 80, 1181.

145. Jones, G. R.; Cundall, R. B.; Murray, D.; Duddell, D. A. J. Chem. Soc., Faraday Trans. II 1984, 80, 1201.

146. Fornasiero, D.; Kurucsev, T. J. Chem. Soc. Faraday Trans. II 1986, $82,15$.

147. Valdes-Aguilera, O.; Neckers, D. C. J. Phys. Chem. 1988, 92, 4286.

148. Ben-Naim, A. J. Chem. Phys. 1989, 90, 7412.

149. Meyer, B. Low Temperature Spectroscopy; American Elsevier Publishing, Inc.: New York, 1971.

150. Breene, R. G., Theories of Spectral Line Shape; John WileyInterscience: New York, 1981.

151. Pusey, P. N.; Trough, R. J. A. In Dynamic Light Scattering; Pecora, R. ed.; Plenum Press: New York and London, 1985.

152. Struve, W. S. Fundamentals of Molecular spectroscopy; John Wiley \& Sons, Inc.: New York, 1989, 267.

153. Byrn, M. P.; Curtis, C. J.; Khan, S. I.; Sawin, P. A.; Tsurumi, R.; Strouse, C. E. J. Am. Chem. Soc. 1990, 112, 1865. 